MÉMOIRES DE LA SMF 97

\title{
INTÉGRALES ORBITALES \\ UNIPOTENTES STABLES ET \\ LEURS TRANSFORMÉES DE SATAKE
}

\author{
Gia-Vuong Nguyen-Chu
}

Société Mathématique de France 2004

Publié avec le concours du Centre National de la Recherche Scientifique 
G.-V. Nguyen-Chu

Max-Planck Institute of Mathematics, Vivatsgasse 7, 53111 Bonn, Germany.

E-mail : ncgvuong@mpim-bonn.mpg.de

Classification mathématique par sujets (2000). - 22E35, 22E50.

Mots clefs. - Analyse harmonique, Groupes réductifs p-adiques, Algèbres de Hecke, Transformation de Satake, Intégrales orbitales unipotentes, Stabilité, Représentations, Traces, Endoscopie tordue. 


\title{
INTÉGRALES ORBITALES UNIPOTENTES STABLES ET LEURS TRANSFORMÉES DE SATAKE
}

\author{
Gia-Vuong Nguyen-Chu
}

Résumé. - Dans cet article, nous abordons quelques questions d'analyse harmonique sur les groupes réductifs $p$-adiques. Plus précisément, nous nous intéressons à la transformation de Satake des distributions unipotentes stables dans le cas des groupes déployés. Ce problème est motivé, d'une part par les travaux de M. Assem sur le calcul des intégrales orbitales unipotentes, et d'autre part par ceux de J.-L. Waldspurger sur la détermination de l'espace des distributions unipotentes stables. Cette question est facile pour les groupes linéaires mais inconnue en général. Dans ce travail, nous traitons le cas des groupes $\mathbf{S p}(2 n)$. Pour $n=2$, nous démontrons que ces transformées de Satake s'expriment comme des fonctions régulières sur le tore réel unitaire de dimension 2. Nous montrons ensuite que ces fonctions peuvent également être retrouvées par la transformation de Satake des distributions de toute autre nature : les traces tordues compactes d'une famille explicite de représentations de $\mathbf{G L}(5)$. Ce phénomène peut s'expliquer par l'endoscopie tordue entre $\mathbf{S p}(2 n)$ et $\mathbf{G L}(2 n+1)$ comme l'a remarqué Arthur. Pour $n>2$, on démontre dans un certain nombre de cas que les transformées de Satake de telles traces sont effectivement des fonctions régulières, d'une forme commune, sur le tore réel unitaire de rang $n$. On l'a en particulier vérifié pour $n \leqslant 4$. On s'attend à ce que ceci reste vrai pour $n$ quelconque. Grâce à ces calculs, on propose alors une conjecture assez précise qui décrit les transformées de Satake des distributions unipotentes stables $\operatorname{sur} \mathbf{S p}(2 n)$. 


\section{Abstract (Stable unipotent orbital integrals and their Satake transforms)}

In this article, we are concerned with some questions arising from harmonic analysis on $p$-adic groups. More precisely, we are interested in Satake transforms of stable unipotent distributions in the case of split groups. This problem is motivated, on one hand, by M. Assem's work on the computation of unipotent orbital integrals, and on the other hand, by J.-L. Waldspurgers' on the determination of the space of stable unipotent distributions. This question is easy for general linear groups but unkown in general. In this work, we deal with the groups $\mathbf{S p}(2 n)$. For $n=2$, we show that these Satake transforms are regular functions over the rank-2 unitary real torus. We then show that these functions can be recovered by the Satake transform of some distributions of a totally different kind: the twisted compact traces of an explicit familly of representations of $\mathbf{G L}(5)$. This phenomenon may be explained by twisted endoscopy between $\mathbf{S p}(2 n)$ and $\mathbf{G L}(2 n+1)$ as remarked by Arthur. For $n>2$, we show, in some cases, that the Satake transforms of these traces are actually regular functions, of a common form, over the rank- $n$ unitary real torus. In particular, we have verified it when $n \leqslant 4$. We expect that it is true in general. Thanks to these computations, we then propose a quite precise conjecture, that describes the Satake transforms of stable unipotent distributions on $\mathbf{S p}(2 n)$. 


\section{TABLE DES MATIÈRES}

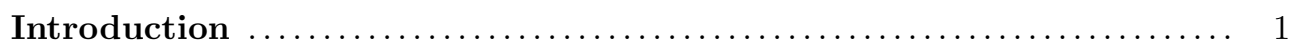

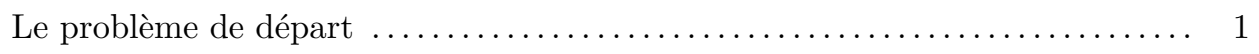

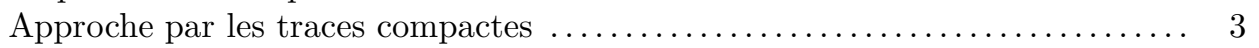

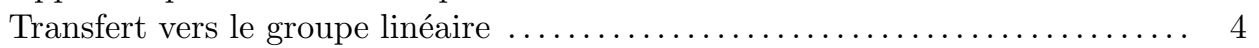

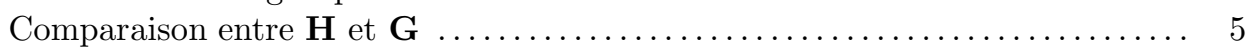

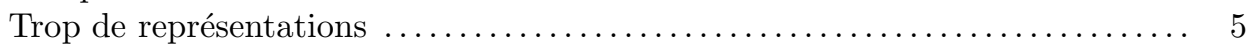

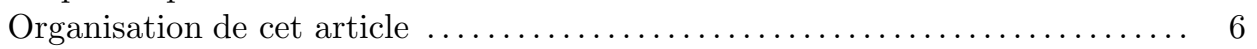

1. Une formule pour les traces tordues compactes $\ldots \ldots \ldots \ldots \ldots \ldots \ldots$

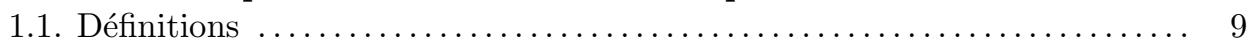

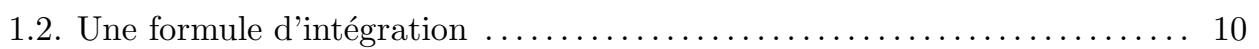

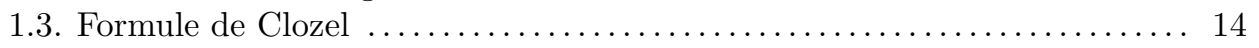

2. Les traces tordues compactes sur $G L(2 n+1) \ldots \ldots \ldots \ldots \ldots \ldots \ldots \ldots \ldots$

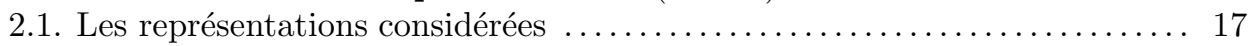

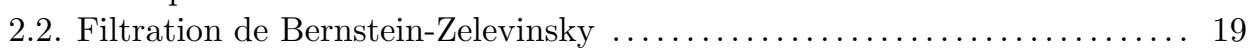

2.3. Normalisation des opérateurs d'entrelacement $\ldots \ldots \ldots \ldots \ldots \ldots \ldots \ldots \ldots 20$

2.4. Premier exemple de calculs, cas où $\pi$ est symétrique $\ldots \ldots \ldots \ldots \ldots \ldots \ldots 26$

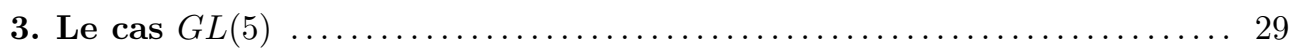

3.1. Calculs explicites dans les cas symétriques $\ldots \ldots \ldots \ldots \ldots \ldots \ldots \ldots \ldots \ldots$

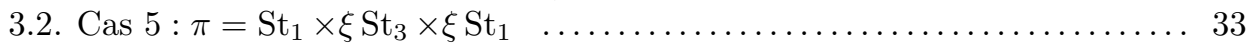

3.3. Transformation de Satake des traces tordues compactes $\ldots \ldots \ldots \ldots \ldots . .39$

4. Intégrales orbitales unipotentes stables $\operatorname{sur} \operatorname{Sp}(4) \ldots \ldots \ldots \ldots \ldots$

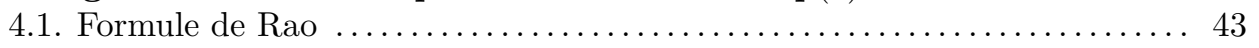

4.2. Distributions stablement invariantes à support unipotent $\ldots \ldots \ldots \ldots \ldots 44$

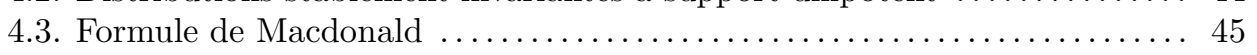

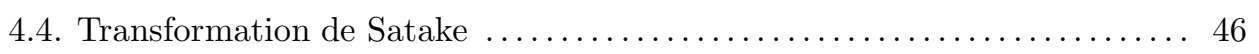

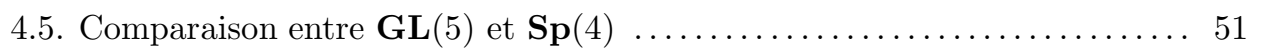


5. Le cas général, réduction au cas où $\beta=\varnothing \ldots \ldots \ldots \ldots \ldots \ldots \ldots \ldots \ldots$

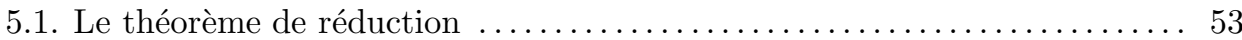

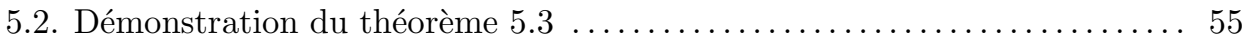

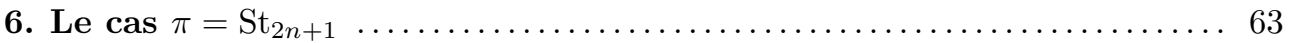

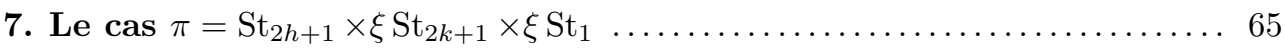

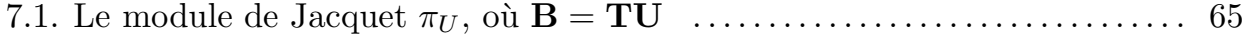

7.2. Les modules de Jacquet $\pi_{N}$ où $\mathbf{P}=\mathbf{M N} \in \mathcal{P}^{\theta-s t} \ldots \ldots \ldots \ldots \ldots \ldots \ldots$

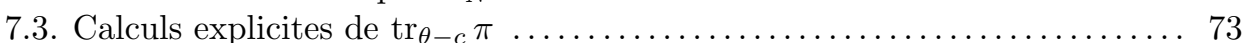

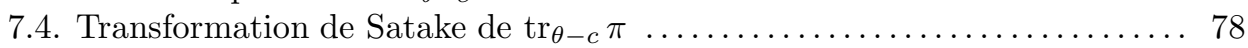

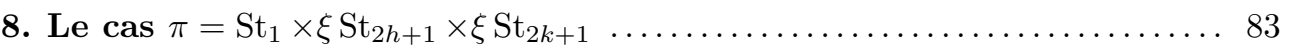

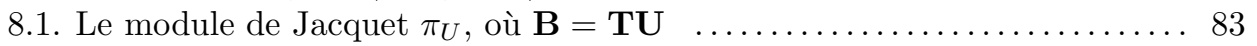

8.2. Les modules de Jacquet $\pi_{N}$ où $\mathbf{P}=\mathbf{M N} \in \mathcal{P}^{\theta} \ldots \ldots \ldots \ldots \ldots \ldots \ldots \ldots$.

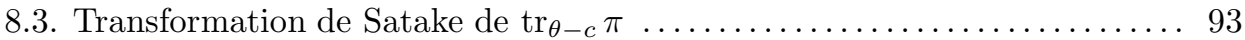

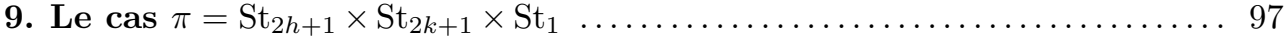

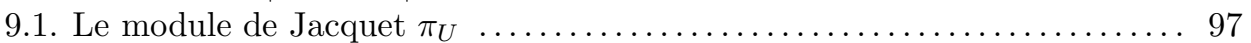

9.2. Les modules de Jacquet $\pi_{N}$, où $\mathbf{P}=\mathbf{M N} \in \mathcal{P}^{\theta} \ldots \ldots \ldots \ldots \ldots \ldots \ldots$

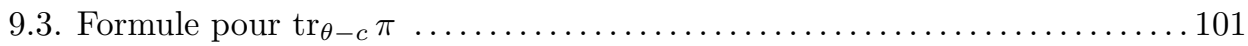

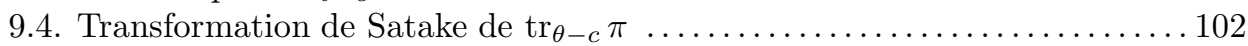

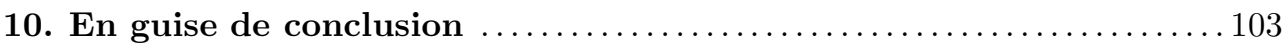

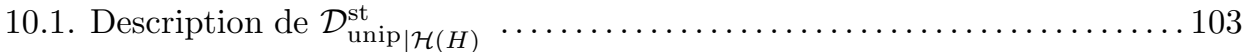

10.2. Analogie avec les traces tordues compactes $\ldots \ldots \ldots \ldots \ldots \ldots \ldots \ldots \ldots \ldots$

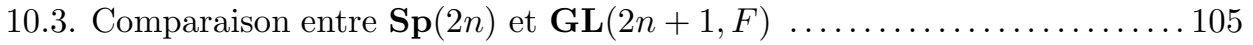

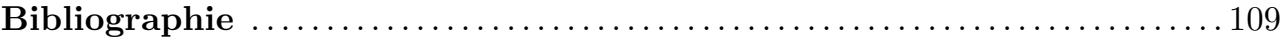




\section{INTRODUCTION}

\section{Le problème de départ}

Soit $\mathbf{H}$ un groupe réductif, connexe, déployé sur un corps local non archimédien $F$ de caractéristique zéro et de corps résiduel $\mathbb{F}_{q}$. Notons $H=\mathbf{H}(F)$, et fixons un sousgroupe compact maximal hyperspécial $K$. Soit $\mathcal{H}(H)$ l'algèbre de Hecke sphérique correspondante. Soient $D_{\text {unip }}, D^{\text {st }}, D_{\text {unip }}^{\text {st }}$ les espaces des distributions invariantes sur $H$ à support unipotent, stables et stables à support unipotent respectivement. Le troisième espace est l'intersection des deux premiers. L'algèbre $\mathcal{H}(H)$ est isomorphe via l'isomorphisme de Satake à une algèbre de polynômes notée $\check{\mathcal{H}}(H)$, alors que l'espace $D_{\text {unip }}$ admet une base paramétrée par les orbites unipotentes de $G$ dont on connaît dans de nombreux cas le paramétrage combinatoire. Ces deux objets sont donc essentiellement de nature combinatoire. L'idée à l'origine de cet article est de comprendre la restriction de $D_{\text {unip à }} \mathcal{H}(H)$. Cependant, on peut se contenter dans un premier temps de se restreindre au sous-espace $D_{\text {unip }}^{\text {st }}$. On espère ensuite pouvoir étendre les

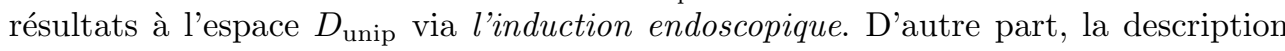
de $D_{\text {unip }}^{\text {st }}$ a été donnée par Waldspurger dans [Wa2], sous l'hypothèse que $\mathbf{H}$ est un groupe classique non ramifié, et la caractéristique résiduelle de $F$ est assez grande, ce que nous supposons par la suite. Il s'agit essentiellement de calculs explicites.

Considérons l'exemple où $\mathbf{H}$ est le groupe linéaire $\mathbf{G L}(n)$. L'algèbre $\breve{\mathcal{H}}(H)$ est alors l'algèbre des polynômes en $n$ variables $X_{1}, \ldots, X_{n}$ ainsi que leurs inverses, qui sont invariants par le groupe symétrique $\mathfrak{S}_{n}$. L'action du groupe $\mathfrak{S}_{n}$ est la permutation des variables $X_{i}$. Dans ce cas, les espaces $D_{\text {unip }}^{\text {st }}$ et $D_{\text {unip }}$ coïncident. Une base de cet espace est donnée par les intégrales orbitales sur les orbites unipotentes de $G L(n)=$ $\mathbf{G L}(n, F)$. Soit $\mathcal{O}$ une orbite unipotente de $G L(n)$. On sait alors qu'il existe un sousgroupe parabolique standard $P=M N$ pour lequel $\mathcal{O}$ est une orbite de Richardson. On associe à $M$ une fonction, dite de Harish-Chandra de la façon suivante. Pour toute racine $\alpha$ du tore diagonal, qui correspond, avec les notations usuelles, disons à $e_{i}-e_{j}$, 
posons

$$
\mathbf{c}_{\alpha}\left(x_{1}, \ldots, x_{n}\right)=\frac{1-q^{-1} x_{i} x_{j}^{-1}}{1-x_{i} x_{j}^{-1}}
$$

On définit $\mathbf{c}_{\mathbf{M}}$, la fonction de Harish-Chandra, de $M$, par la formule :

$$
\mathbf{c}_{\mathbf{M}}\left(x_{1}, \ldots, x_{n}\right)=\prod_{\alpha} \mathbf{c}_{\alpha}\left(x_{1}, \ldots, x_{n}\right)
$$

où le produit porte sur toutes les racines $\alpha$ contenues dans l'algèbre de Lie de $M$. On a alors l'identité suivante, qui est due à Macdonald. Pour tout $f \in \mathcal{H}(G L(n))$,

$$
\int_{\mathcal{O}} f=* \int_{\widehat{T}_{u}} S(f)\left(x_{1}, \ldots, x_{n}\right) \mathbf{c}_{\mathbf{M}}\left(x_{1}, \ldots, x_{n}\right)^{-1} d^{\times} x_{1} \cdots d^{\times} x_{n}
$$

où le membre de gauche est l'intégrale orbitale sur $\mathcal{O}$ de $f, S$ désigne l'isomorphisme de Satake, $\widehat{T}_{u}=\left\{\left(x_{1}, \ldots, x_{n}\right) \in \mathbb{C}^{n} ;\left|x_{1}\right|=\cdots=\left|x_{n}\right|=1\right\}$, et enfin $*$ est une constante. On peut donc dire que la transformée de Satake d'un élément de $D_{\text {unip }}^{\text {st }}$ est donnée par (l'intégration sur $\widehat{T}_{u}$ contre) une fonction rationnelle en $n$-variables. Signalons au passage que dans ce cas les fonctions $\mathbf{c}_{\mathbf{M}}$ sont totalement explicites.

Pour $\mathbf{H}$ général, on espère qu'il existe une formule analogue. L'objet de cet article est de démontrer quelques résultats et de comprendre un peu $D_{\text {unip } \mid \mathcal{H}(H)}^{\mathrm{st}}$ lorsque le groupe $\mathbf{H}$ est un groupe symplectique. Supposons désormais que $\mathbf{H}=\mathbf{S p}(2 n)$. Précisons un peu à quoi on peut s'attendre. Nous allons avoir besoin de la description de $D_{\text {unip }}^{\text {st }}$ pour le groupe symplectique. La référence est [Wa2] dont nous reprenons librement les notations. Pour $n, k$ deux entiers positifs, notons $S_{n, k}$, resp. $S_{n, \text { imp }}$, l'ensemble des symboles de rang $n$ et de défaut $k$, resp. de défaut impair. On a : $S_{n, \text { imp }}=\cup_{k(k+1) \leqslant n} S_{n, 2 k+1}$. Notons $\mathcal{I}^{\text {st }}(n)$ l'ensemble des couples $(k, \rho)$ où :

$-k$ est un entier naturel tel que $k(k+1) \leqslant n$;

- $\rho$ est une représentation irréductible de $W\left(C_{n-k(k+1)}\right)$ - le groupe de Weyl de type $C_{n-k(k+1)}$.

Pour $k$ fixé, notons $\mathcal{I}_{k}^{\text {st }}(n)$ l'ensemble des tels couples dont le premier facteur est égal à $k$. On dispose alors d'une bijection combinatoire :

$$
S_{n, \mathrm{imp}} \longleftrightarrow \mathcal{I}^{\mathrm{st}}(n)
$$

de sorte que pour tout $k, k(k+1) \leqslant n$,

$$
S_{n, 2 k+1} \longleftrightarrow \mathcal{I}_{k}^{\mathrm{st}}(n)
$$

Dans [Wa2], l'auteur construit une base de $D_{\text {unip }}^{\text {st }}$ que nous allons noter $\mathcal{B}$. Cette base est paramétrée par $S_{n, \text { imp }}$ (et donc par $\mathcal{I}^{\text {st }}(n)$, d'après ce qui précède). Pour $(k, \rho) \in \mathcal{I}^{\text {st }}(n)$, soit $I_{k, \rho}$, l'élément de $\mathcal{B}$ correspondant. Voici ce que nous pouvons attendre de $\mathcal{D}_{\text {unip } \mid \mathcal{H}(H)}^{\text {st }}$ : 


\section{RÉSULTATS ESPÉRÉS 1}

(1) -Annulation-Pour tout $(k, \rho) \in \mathcal{I}^{\text {st }}(n)$ tel que $k \neq 0$, la restriction de $I_{k, \rho} \grave{a}$ $\mathcal{H}(H)=\mathcal{H}(S p(2 n))$ est nulle.

(2) -Base proposée pour $\mathcal{D}_{\text {unip } \mid \mathcal{H}(H)}^{\text {st }}$ Les restrictions à $\mathcal{H}(H)$ des éléments $I_{0, \rho}$, $(0, \rho) \in \mathcal{I}_{0}^{\text {st }}(n)$ forment une base de $\mathcal{D}_{\text {unip } \mid \mathcal{H}(H)}^{\text {st }}$

(3) -Transformées de Satake des éléments de $\mathcal{B}-$ En plus, pour tout $(0, \rho) \in \mathcal{I}_{0}^{\text {st }}(n)$, il existe une fonction rationnelle $c_{\rho}$ en $n$ variables, invariante par le groupe de Weyl $W\left(C_{n}\right)$, sans pôle au voisinage de $\widehat{T}_{u}$, telle que pour tout $f \in \mathcal{H}(H)$, on ait l'égalité :

$$
I_{0, \rho}(f)=\int_{\widehat{T}_{u}} S(f)\left(x_{1}, \ldots, x_{n}\right) c_{\rho}\left(x_{1}, \ldots, x_{n}\right) d^{\times} x_{1} \cdots d^{\times} x_{n} .
$$

Ces énoncés ont été démontrés dans cet article pour $n=2$. La preuve s'appuie essentiellement sur les calculs de Assem dans [As2]. Pour $n>2$, on n'a malheureusement pas assez de résultats sur les intégrales orbitales unipotentes des éléments de l'algèbre de Hecke sphérique.

\section{Approche par les traces compactes}

Dans cet article, comme on a déjà dit au début de cette introduction, il n'est pas question de calculer les fonctions $c_{\rho}$, mais plutôt de donner une description de l'espace engendré par celles-ci. Comment donc trouver des fonctions susceptibles d'engendrer cet espace? Par analogie, il semble naturel de penser aux distributions traces sur $H$, ou plus précisément aux traces compactes. Pour $\pi$ une représentation lisse, irréductible de $H$, soit $\operatorname{tr}_{c} \pi$ la distribution trace compacte correspondante (voir le chapitre suivant pour une définition précise). Notre conviction d'utiliser ces distributions provient, entre autres, d'un résultat de Courtès :

$$
\mathcal{D}_{\text {unip } \mid \mathcal{H}(H)}=\mathbb{C}\left\{\operatorname{tr}_{c} \pi ; \pi \text { tempérée }\right\}_{\mid \mathcal{H}(H)}
$$

l'espace engendré par les restrictions à $\mathcal{H}(H)$ des traces compactes des représentations tempérées de $H$.

Par application d'une formule de Clozel ( $c f$. formule 1.7 pour $\theta$ trivial), il suffit de considérer les sous-quotients d'une série principale non ramifiée; les autres étant de restriction nulle sur $\mathcal{H}(H)$. D'autre part, il s'agit ici de distributions stables, il faut donc regrouper les représentations en des $L$-paquets. On est ainsi amené à calculer des traces compactes des $L$-paquets tempérés, et contenant un sous-quotient d'une série principale non-ramifiée. Mais là encore, on comprend assez mal ces objets. En tout cas, on n'est pas tenté de calculer ces traces mais plutôt de ramener ces objets sur un terrain plus familier avant d'effectuer les calculs nécessaires. 


\section{Transfert vers le groupe linéaire}

On note $\mathbf{G}=\mathbf{G} \mathbf{L}(2 n+1)$ que l'on munit d'une involution $\theta$ dont la définition est donnée par la formule 2.1. Posons : $\widetilde{\mathbf{G}}=\mathbf{G} \rtimes\{1, \theta\}$. Comme l'a remarqué Arthur, le groupe $\mathbf{H}$ est alors un groupe endoscopique tordu de $\widetilde{\mathbf{G}}$, au sens de Kottwitz-Shelstad [K-S]. Nous n'entrons pas dans les détails mais notons simplement que le groupe dual de $\mathbf{H}$, le groupe $\mathbf{S O}(2 n+1, \mathbb{C})$, est la composante neutre du centralisateur dans $\mathbf{G L}(2 n+1, \mathbb{C}) \rtimes\{1, \theta\}$ de l'élément

$$
\operatorname{diag}(1,-1,1,-1, \ldots, 1) \rtimes \theta
$$

La philosophie de Langlands suggère alors un transfert des $L$-paquets en question sur $H$ vers des $L$-paquets sur $G$. Remarquons que pour le groupe linéaire chaque $L$-paquet est constitué d'une seule représentation. Il s'agit de l'endoscopie tordue, on est amené à calculer les traces tordues compactes de telles représentations sur $\mathcal{H}(G)$, l'algèbre de Hecke sphérique de $G$. La définition de la trace tordue compacte se trouve au premier chapitre. Explicitons l'ensemble des représentations de $G$ qui nous intéressent. Posons :

$$
\mathcal{A}^{\text {st }}(n)=\left\{\begin{array}{c}
\alpha^{+}, \alpha^{-}, \beta \text { des partitions telles que : } \\
\bullet \\
\bullet\left(\alpha^{+}\right)+S\left(\alpha^{-}\right)+2 S(\beta)=2 n+1 ; \\
\left(\alpha^{+}, \alpha^{-}, \beta\right) ; \text { les partitions } \alpha^{+}, \alpha^{-} \text {sont constituées } \\
\text { de nombres impairs, de multiplicité } 1 ; \\
\bullet \text { le nombre de termes de } \alpha^{-} \text {est pair } ;
\end{array}\right\}
$$

où on a noté $S\left(\alpha^{+}\right)$pour la somme des termes de la partition $\alpha^{+}$, idem pour $S\left(\alpha^{-}\right), S(\beta)$. L'ensemble des représentations de $G$ qui nous intéressent est alors paramétré par :

- un triplet $\left(\alpha^{+}=\left(\alpha_{1}^{+}, \ldots, \alpha_{r}^{+}\right), \alpha^{-}=\left(\alpha_{1}^{-}, \ldots, \alpha_{s}^{-}\right), \beta=\left(\beta_{1}, \ldots, \beta_{t}\right)\right) \in \mathcal{A}^{\text {st }}(n)$;

- des nombres complexes $z_{1}, \ldots, z_{t}$.

Pour $k_{1}, k_{2}$ deux entiers strictement positifs, $\pi_{1}, \pi_{2}$ deux représentations de $G L\left(k_{1}\right), G L\left(k_{2}\right)$ respectivement, nous notons $\pi_{1} \times \pi_{2}$ l'induite parabolique normalisée de $\pi_{1} \otimes \pi_{2}$ à $G L\left(k_{1}+k_{2}\right)$. Soit $\left(\alpha^{+}, \alpha^{-}, \beta, z\right)$ un tel paramètre. La représentation que l'on associe à ces données est

$$
\begin{array}{r}
\pi^{\prime}=|\cdot|{ }^{z_{1}} \mathrm{St}_{\beta_{1}} \times \cdots \times \mid \cdot{ }^{z_{t}} \mathrm{St}_{\beta_{t}} \times \mathrm{St}_{\alpha_{1}^{+}} \times \cdots \times \mathrm{St}_{\alpha_{r}^{+}} \times \xi \mathrm{St}_{\alpha_{1}^{-}} \times \cdots \times \xi \mathrm{St}_{\alpha_{s}^{-}} \\
\times|\cdot|^{-z_{t}} \mathrm{St}_{\beta_{t}} \times \cdots \times|\cdot|^{-z_{1}} \mathrm{St}_{\beta_{1}}
\end{array}
$$

où $\mathrm{St}_{m}$ désigne la représentation de Steinberg de $G L(m), \xi$ est le caractère quadratique non ramifié non trivial de $F^{\times}$. Notons $\pi$ la représentation :

$$
\pi=\mathrm{St}_{\beta_{1}} \times \cdots \times \mathrm{St}_{\beta_{t}} \times \mathrm{St}_{\alpha_{1}^{+}} \times \cdots \times \mathrm{St}_{\alpha_{r}^{+}} \times \xi \mathrm{St}_{\alpha_{1}^{-}} \times \cdots \times \xi \mathrm{St}_{\alpha_{s}^{-}} \times \mathrm{St}_{\beta_{t}} \times \cdots \times \mathrm{St}_{\beta_{1}}
$$

On peut alors montrer que pour tout $f \in \mathcal{H}(G)$,

$$
\operatorname{tr}_{\theta-c} \pi(f)=\operatorname{tr}_{\theta-c} \pi^{\prime}(f)
$$


Ce fait peut se déduire de la démonstration de la réduction au cas $\beta=\varnothing$ ( $c f$. chapitre 5). Ainsi, pour notre propos, on peut oublier les nombres complexes $z_{i}$ de sorte que les représentations $\pi$ considérées sont paramétrées par l'ensemble $\mathcal{A}^{\text {st }}(n)$ défini plus haut.

Comme $\mathbf{H}$ est un groupe endoscopique pour $\widetilde{\mathbf{G}}$, on dispose alors d'un homomorphisme d'algèbres :

$$
p: \mathcal{H}(G) \longrightarrow \mathcal{H}(H)
$$

La définition de $p$ est donnée par la formule 5.1, qui n'est qu'une interprétation simple dans notre cas d'une situation générale.

Somme toute, nous disposons des distributions

$$
f \in \mathcal{H}(G) \longmapsto \operatorname{tr}_{\theta-c} \pi(f)
$$

qui, on a déjà dit, sont paramétrées par l'ensemble $\mathcal{A}^{\text {st }}(n)$. Ce que l'on attend de ces distributions se trouve dans :

RÉSultats ESPÉRÉs 2. - Pour tout $\left(\alpha^{+}, \alpha^{-}, \beta\right) \in \mathcal{A}^{\mathrm{st}}(n)$, soit $\pi$ la représentation correspondante, il existe une fonction rationnelle $c_{\pi}$ en $n$ variables, invariante par le groupe de Weyl $W\left(C_{n}\right)$, sans pôle sur le tore $\widehat{T}_{u}$ telle que pour tout élément $f$ de l'algèbre de Hecke de $G=G L(2 n+1)$ on ait l'identité :

$$
\operatorname{tr}_{\theta-c} \pi(f)=\int_{\widehat{T}_{u}} p(S(f))\left(x_{1}, \ldots, x_{n}\right) c_{\pi}\left(x_{1}, \ldots, x_{n}\right) d^{\times} x_{1} \cdots d^{\times} x_{n} .
$$

Le travail essentiel de cet article a été de démontrer cet énoncé dans les cas où $\beta=\varnothing, \alpha=\alpha^{+} \cup \alpha^{-}$a soit un terme, soit trois termes dont l'un est égal à 1 . On a en particulier vérifié cet énoncé pour $n=1,2,3,4$.

\section{Comparaison entre $\mathbf{H}$ et $\mathbf{G}$}

L'existence et la forme des fonctions rationnelles $c_{\pi}$ sont a priori plus simples à deviner. On donnera effectivement une forme espérée de ces fonctions à la fin de cet article. Rappelons que notre but de départ était d'avoir des renseignements sur $D_{\text {unip } \mid \mathcal{H}(H)}^{\text {st }}$. La comparaison entre les groupes $\mathbf{H}$ et $\mathbf{G}$ est l'énoncé conjectural suivant :

RÉSUlTATS ESPÉRÉS 3. - Les espaces engendrés par les fonctions $c_{\pi}$ et $c_{\rho}$ coïncident.

On trouvera dans ce travail une réponse positive à cette question dans le cas $n=2$.

\section{Trop de représentations}

Nous pouvons chercher à construire une base de l'espace engendré par les fonctions $c_{\rho}$ en termes des fonctions $c_{\pi}$. Comme nous le montrerons dans cet article, l'ensemble $\mathcal{A}^{\text {st }}(n)$ a un plus grand nombre d'éléments que l'ensemble $\mathcal{I}_{0}^{\text {st }}(n)$. On est 
conduit à restreindre l'ensemble $\mathcal{A}^{\text {st }}(n)$ en un sous-ensemble $\mathcal{A}_{0}^{\text {st }}(n)$ (voir le dernier chapitre de cet article), qui est de même cardinal que $\mathcal{I}_{0}^{\text {st }}(n)$. On espère que l'énoncé suivant est vérifié :

RÉSUltats EsPÉRÉS 4. - Lorsque les éléments $\left(\alpha^{+}, \alpha^{-}, \beta\right)$ parcourent $\mathcal{A}_{0}^{\mathrm{st}}(n)$, les fonctions $c_{\pi}$ forment une base de l'espace engendré par toutes les fonctions $c_{\pi}$.

On obtient ainsi une description conjecturale assez satisfaisante de $D_{\text {unip } \mid \mathcal{H}(H)}^{\text {st }}$ dans le cas du groupe symplectique.

\section{Organisation de cet article}

Dans le premier chapitre, nous définissons la notion de trace compacte et trace tordue compacte. Le résultat principal de cette section est la formule suivante de Clozel. Soit $\mathbf{G}$ un groupe réductif, connexe, déployé sur $F$, muni d'une involution $\theta$ vérifiant certaines hypothèses. Soit $(\pi, V)$ une représentation lisse, irréductible et $\theta$-stable de $G=\mathbf{G}(F)$. Soit $A \in \operatorname{End}_{\mathbb{C}}(V)$ un opérateur non nul qui entrelace $\pi$ et $\pi^{\theta}$. On note $\mathcal{P}^{\theta}$, l'ensemble des paraboliques standard $\theta$-stables de $G$. Pour $P=M N \in$ $\mathcal{P}^{\theta}$, l'action de $A$ se descend en une action sur le module de Jacquet $\left(\pi_{N}, V_{N}\right)$. On peut donc définir la trace tordue $\operatorname{tr}_{\theta} \pi_{N}$. Le résultat principal est le théorème 1.6 dont la formule 1.7 calcule la trace tordue compacte $\operatorname{tr}_{\theta-c} \pi$ en une somme sur les $P=M N \in \mathcal{P}^{\theta}$ des traces tordues $\operatorname{tr}_{\theta} \pi_{N}$. La démonstration de ce résultat est due à Clozel (sous d'autres formes). Cependant, par souci de complétude, on reprend sa démonstration en indiquant ce qu'il fallait changer dans la preuve.

Dans le deuxième chapitre, on introduit les représentations $\pi$ qui nous intéressent et quelques définitions. On y rappelle la filtration de Bernstein-Zelevinsky qui sera très utile pour notre étude. Mais le point essentiel de ce chapitre est la normalisation de l'opérateur $A: \pi \simeq \pi^{\theta}$. La normalisation est la suivante. Soit $V$ l'espace de $\pi$, soit $I$ le sous-groupe d'Iwahori usuel de $G$. On montre que $V^{I}$, les invariants par $I$, contient une droite $D$ bien définie, qui est stable par $A$. On normalise alors $A$ de sorte qu'il fixe $D$. On y donne alors un premier exemple de calcul, le cas où $\pi$ est symétrique de sorte que l'action de $A$ soit très simple ( $c f$. formule 2.8). La méthode est d'utiliser la filtration de Bernstein-Zelevinsky pour déterminer l'action de $A$ sur les modules de Jacquet (ou plus précisément sur les parties qui contribuent à la trace). Une fois ces actions déterminées, il ne reste qu'à appliquer la formule de Van Dijk pour avoir le terme correspondant à un parabolique $\theta$-stable donné $c f$. formule 2.13 .

Dans le troisième chapitre, on étudie le cas $\mathbf{G L}(5)$ avec les représentations décrites ci-dessus. Au total, il y a cinq cas à étudier. Parmi ceux ci, quatre sont de la forme symétrique et on applique les résultats du deuxième chapitre. Le dernier des cinq cas est traité à part. Nous montrons que l'opérateur $A$ n'est pas d'une forme simple comme dans les cas précédents mais est, grosso modo, une dérivée des opérateurs 
d'entrelacement proprement dits. Des études manuelles permettent, malgré la définition compliquée de $A$, d'identifier son action sur les modules de Jacquet, et du coup, nous obtenons aussi une formule explicite pour $\operatorname{tr}_{\theta-c} \pi(f)$ ( $c f$. Prop. 3.17). Grâce à ces calculs, nous déterminons les fonctions rationnelles $c_{\pi}$ déjà évoquées ( $c f$. Th. 3.18). Signalons que M. Reeder a fait des calculs analogues dans [Ree].

Le quatrième chapitre est consacré aux intégrales orbitales sur $\mathbf{H}=\mathbf{S p}(4)$. Nous aurions pu utiliser directement les calculs de Assem dans [As2]. Cependant, en lisant [As2] nous avons trouvé quelques erreurs. Par prudence, nous avons préféré reprendre les calculs. Plusieurs orbites sont des orbites de Richardson dans $H$ pour lesquelles les calculs des intégrales orbitales sont faciles. Il ne reste qu'une seule intégrale orbitale unipotente stable à calculer. Au cours des calculs nous avons apporté quelques simplifications mineures. On détermine ensuite les fonctions rationnelles $c_{\rho}$. La comparaison entre $H$ et $G$ est alors complète et correspond bien à nos espérances. L'espace engendré par les fonctions $c_{\rho}$ coiincide effectivement avec celui engendré par les fonctions $c_{\pi}$. Nous exprimons explicitement, sous forme des matrices de changement de base, les fonctions $c_{\rho}$ en termes des $c_{\pi}$ et vice versa.

Dans le cinquième chapitre, nous nous intéressons au cas général $n \geqslant 1$. Cette étude concerne seulement les représentations de $G$. Le résultat principal de ce chapitre est la proposition 5.3, qui permet de réduire nos études aux représentations paramétrées par les triplets $\left(\alpha^{+}, \alpha^{-}, \beta\right) \in \mathcal{A}_{0}^{\text {st }}(n)$ avec $\beta=\varnothing$.

Dans les chapitres $6,7,8$ et 9 , nous vérifions les RÉSULtATs EsPÉRÉs 2 pour $\pi$ dans quatre cas suivants :

(1) $\mathrm{St}_{2 n+1}$;

(2) $\mathrm{St}_{2 h+1} \times \xi \mathrm{St}_{2 k+1} \times \xi \mathrm{St}_{1}, \quad h+k=n-1, k \geqslant 1$;

(3) $\mathrm{St}_{1} \times \xi \mathrm{St}_{2 h+1} \times \xi \mathrm{St}_{2 k+1}, \quad h+k=n-1, h>k$;

(4) $\mathrm{St}_{2 h+1} \times \mathrm{St}_{2 k+1} \times \mathrm{St}_{1}, \quad h+k=n-1, h>k \geqslant 1$.

Les résultats principaux sont les théorèmes $6.2,7.39,8.25$ et 9.7 . Comme conséquence facile de ces calculs, on montre que les RÉSultats ESPÉRÉs 2 sont vérifiés pour $n=1,2,3,4$. On remarquera également que les principaux résultats sur $G L(5)$ peuvent se déduire de ces calculs.

Enfin, on conclut avec le chapitre 10 dans lequel nous essayons de généraliser nos résultats sous des formes conjecturales. Plus précisément, en admettant l'existence des fonctions $c_{\pi}$ ( $c f$. RÉSultats ESPÉRÉS 2), on en donne conjecturalement une expression combinatoire simple. Cette expression généralise les résultats obtenus dans les chapitres précédents. Notons que, modulo les RÉsultats espéRÉs 1 et 3, décrire les fonctions $c_{\pi}$ permettent de décrire l'espace $D_{\mathrm{unip} \mid \mathcal{H}(H)}^{\mathrm{st}}$. 



\section{CHAPITRE 1}

\section{UNE FORMULE POUR LES TRACES TORDUES COMPACTES}

\subsection{Définitions}

1.1.1. Traces tordues. - Soit $\mathbf{G}$ un groupe réductif, connexe, déployé sur $F$, muni d'une involution $\theta$. On suppose que celle-ci laisse stable un couple $(\mathbf{T}, \mathbf{B})$ que l'on fixera, où $\mathbf{T}$ est un tore déployé maximal et $\mathbf{B}$ un sous-groupe de Borel de $\mathbf{G}$ contenant T. Remarquons qu'une telle involution conserve l'ensemble des racines simples de $\mathbf{T}$ associé à $\mathbf{B}$ dans l'algèbre de Lie de $\mathbf{G}$. Elle respecte donc la longueur sur le système de racines de $\mathbf{G}$.

Soit $(\pi, V)$ une représentation lisse, admissible, et irréductible de $G=\mathbf{G}(F)$. On dit qu'elle est $\theta$-stable s'il existe un opérateur $A \in \operatorname{End}_{\mathbb{C}}(V)$ non nul, qui est alors unique à un scalaire près, tel que pour tout $g \in G$, on ait l'égalité : $A \circ \pi(g)=\pi(\theta(g)) \circ A$. Supposons $(\pi, V) \theta$-stable et choisissons un tel $A$. Notons $C_{c}^{\infty}(G)$ l'ensemble des fonctions localement constantes, à support compact sur $G$. On note $\operatorname{tr}_{\theta} \pi$ l'application suivante :

$$
f \in C_{c}^{\infty}(G) \longmapsto \operatorname{tr}_{\theta} \pi(f)=\operatorname{trace}\left(\int_{G} A \circ \pi(g) \cdot f(g) d g\right)
$$

On appelle cette distribution la trace tordue (relativement à $\theta$ ) de $\pi$.

1.1.2. Éléments compacts, traces tordues compactes. - Posons $\widetilde{G}=$ $G \rtimes\{1, \theta\}$. Pour $g \in G$, posons $\widetilde{g}=g \rtimes \theta$. Le groupe $\widetilde{G}$ opère par conjugaison sur $G: \operatorname{Ad} \widetilde{g}=\operatorname{Ad} g \circ \theta$. Dorénavant, on emploiera des lettres gothiques pour désigner les algèbres de Lie, par exemple $\mathfrak{G}$ désignera l'algèbre de Lie de $\mathbf{G}$. Soit $g \in G$, notons par $\Gamma_{g}$ l'ensemble des valeurs propres de Ad $\widetilde{g}$ dans $\mathfrak{G}$. Pour $\alpha \in \Gamma_{g}$, posons $\mathfrak{G}_{\alpha}=\{X \in \mathfrak{G} ; \operatorname{Ad} \widetilde{g}(X)=\alpha X\}$ de sorte que : $\mathfrak{G}=\oplus_{\alpha \in \Gamma} \mathfrak{G}_{\alpha}$. On pose :

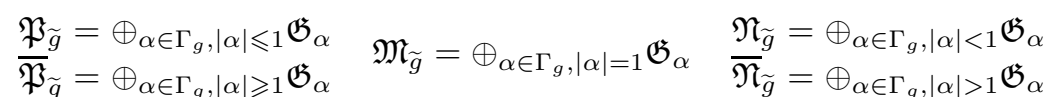

Notons $\mathbf{P}_{\widetilde{g}}, \overline{\mathbf{P}}_{\widetilde{g}}, \mathbf{M}_{\widetilde{g}}, \mathbf{N}_{\widetilde{g}}, \overline{\mathbf{N}}_{\widetilde{g}}$ les sous-groupes de $\mathbf{G}$ correspondants. On a alors : $\mathbf{P}_{\widetilde{g}}=\mathbf{M}_{\tilde{g}} \mathbf{N}_{\tilde{g}}, \overline{\mathbf{P}}_{\widetilde{g}}=\mathbf{M}_{\tilde{g}} \overline{\mathbf{N}}_{\tilde{g}}$. Ce sont des sous-groupes paraboliques de $\mathbf{G}$. Par 
construction $P_{\widetilde{g}}$, resp. $N_{\widetilde{g}}$ est contractant, resp. strictement contractant sous l'action adjointe de $\widetilde{g}$, tandis que $\bar{P}_{\widetilde{g}}$, resp. $\bar{N}_{\widetilde{g}}$ est dilatant, resp. strictement dilatant sous la même action. On vérifie que pour $h \in G$ on a $: \mathbf{P}_{\operatorname{Ad} h(\widetilde{g})}=\operatorname{Ad} h\left(\mathbf{P}_{\widetilde{g}}\right)$. Posons :

$$
G_{\theta-c}=\left\{g \in G ; P_{\widetilde{g}}=G\right\}
$$

C'est l'ensemble des éléments $\theta$-compacts de $G$. Il est invariant par $\theta$-conjugaison. Lorsque $\theta$ est trivial, la formule précédente définit alors l'ensemble $G_{c}$ des éléments compacts de $G$. On remarquera que $g$ est $\theta$-compact si et seulement si $g \theta(g)$ est compact. L'ensemble $G_{\theta-c}$ est un ouvert et fermé de $G$.

Cette construction s'applique au cas où on remplace $\mathbf{G}$ par un sous-groupe de Lévi $\theta$-stable. On définit la trace tordue compacte par la formule :

$$
\operatorname{tr}_{\theta-c} \pi(f)=\operatorname{trace}\left(\int_{G_{\theta-c}} A^{\theta} \circ \pi(g) \cdot f(g) d g\right)
$$

1.1.3. Éléments positifs. - Notons $\mathcal{P}$ l'ensemble des sous-groupes paraboliques de $\mathbf{G}$ contenant $\mathbf{B}$, les sous-groupes paraboliques standard. Pour chaque $\mathbf{P} \in \mathcal{P}$, il existe une unique décomposition de Lévi $\mathbf{P}=\mathbf{M N}$ telle que $\mathbf{T} \subset \mathbf{M}$ et $\mathbf{N}$ soit le radical unipotent de $\mathbf{P}$. Notons $\overline{\mathbf{P}}=\mathbf{M} \overline{\mathbf{N}}$ le sous-groupe parabolique standard opposé à $\mathbf{P}$. Notons $\mathcal{P}^{\theta}$ l'ensemble des éléments de $\mathcal{P}$ qui sont stables par $\theta$. Pour $\mathbf{P}=\mathbf{M N} \in \mathcal{P}^{\theta}$, on a : $\theta(\mathbf{M})=\mathbf{M}, \theta(\mathbf{N})=\mathbf{N}$. Posons :

$$
M_{\theta-c}^{+}=\left\{g \in M ; P_{\widetilde{g}}=P\right\}=\left\{g \in M ; \bar{P}_{\widetilde{g}}=\bar{P}\right\}
$$

C'est l'ensemble des éléments positifs de $M$. On a : $M_{\theta-c}^{+} \subset M_{\theta-c}$, et ces ensembles sont invariants par $\theta$-conjugaison par les éléments de $M$.

\subsection{Une formule d'intégration}

Soit $K$ un sous-groupe compact maximal de $G$. Soient $d g, d k$ les mesures de Haar de $G$ et $K$ qui donnent 1 le volume de $K$. Plus généralement, soit $\mathbf{P}=\mathbf{M N}$ un parabolique standard de $\mathbf{P}$, alors on note $d m$ la mesure de Haar de $M$ qui donne 1 le volume de $K \cap M$. On définit successivement $d p, d n$, les mesures de Haar à gauche sur $P, N$ respectivement, par les formules : $d g=d p d k, d p=d m d n$.

1.2.1. La formule d'intégration entrevue. - Fixons un sous-groupe parabolique standard, $\theta$-stable $\mathbf{P}=\mathbf{M N}$. Posons :

$$
\theta-\operatorname{Ad}(G)\left(M_{\theta-c}^{+}\right)=\left\{g m \theta\left(g^{-1}\right), g \in G, m \in M_{\theta-c}^{+}\right\}
$$

Pour $m \in M_{\theta-c}^{+}$, on pose :

$$
D_{\theta}(m)=\left|\operatorname{det}(\operatorname{Ad} m \circ \theta-1)_{\mid \overline{\mathfrak{N}}}\right|^{-1}
$$

Comme $m \in M_{\theta-c}^{+}$, les valeurs propres de $\operatorname{Ad} m \circ \theta$ dans $\overline{\mathfrak{N}}$ sont de module supérieur à 1 , on a : $D_{\theta}(m)=\left|\operatorname{det}(\operatorname{Ad} m \circ \theta)_{\mid \overline{\mathfrak{N}}}\right|^{-1}=\left|\operatorname{det}(\operatorname{Ad} m \circ \theta)_{\mid \mathfrak{N}}\right|$. 
On va prouver la formule suivante :

Proposition 1.1. - Pour tout $f \in C_{c}^{\infty}(G)$, on a l'égalité :

$$
\int_{\theta-\operatorname{Ad}(G)\left(M_{\theta-c}^{+}\right)} f(g) d g=\int_{M_{\theta-c}^{+} \times N \times K} D_{\theta}(m) f\left(k n m \theta\left(n^{-1}\right) \theta\left(k^{-1}\right)\right) d k d n d m
$$

Démonstration. - Soit $d \bar{n}$ la mesure de Haar à gauche sur $\bar{N}$ telle que $d g=d m d n d \bar{n}$. L'égalité cherchée est équivalente à

$$
\int_{\theta-\operatorname{Ad}(G)\left(M_{\theta-c}^{+}\right)} f(g) d g=\int_{M_{\theta-c}^{+} \times \bar{N} \times N} D_{\theta}(m) f\left(u n m \theta\left(n^{-1}\right) \theta\left(u^{-1}\right)\right) d n d u d m
$$

Montrons cette égalité. D'après la décomposition de la grosse cellule, $G=M \times \bar{N} \times N$ à un ensemble de mesure nulle près. Puisque $M_{\theta-c}^{+}$est stable par $\theta$-conjugaison par les éléments de $M$, on a :

$$
\theta-\operatorname{Ad}(G)\left(M_{\theta-c}^{+}\right)=\left\{u n m \theta\left(n^{-1}\right) \theta\left(u^{-1}\right) ; u \in \bar{N}, m \in M_{\theta-c}^{+}, n \in N\right\}
$$

à un ensemble de mesure nulle près. Considérons l'application :

$$
\begin{aligned}
\phi: M_{\theta-c}^{+} \times \bar{N} \times N & \longrightarrow \theta-\operatorname{Ad}(G)\left(M_{\theta-c}^{+}\right) \subset G \\
(m, u, n) & \longmapsto g=u n m \theta\left(n^{-1}\right) \theta\left(u^{-1}\right)
\end{aligned}
$$

Il s'agit donc de montrer que :

Assertion 1. - Le degré de $\phi$ est 1.

Assertion 2. - Le jacobien de $\phi$ est $d g / d m d u d n=D_{\theta}(m)$.

1.2.2. Preuve de l'assertion 1. - Soient $m, m^{\prime} \in M_{\theta-c}^{+}, n, n^{\prime} \in N, u, u^{\prime} \in \bar{N}$ tels que $\phi(m, u, n)=\phi\left(m^{\prime}, u^{\prime}, n^{\prime}\right)$, c'est-à-dire :

$$
\text { unm } \theta\left(n^{-1}\right) \theta\left(u^{-1}\right)=u^{\prime} n^{\prime} m^{\prime} \theta\left(n^{\prime-1}\right) \theta\left(u^{\prime-1}\right)
$$

Il faut montrer que $m=m^{\prime}, u=u^{\prime}, n=n^{\prime}$. Posons : $x=u n, x^{\prime}=u^{\prime} n^{\prime}$ et $y=x^{\prime-1} x$. On peut alors écrire l'égalité 1.2 sous la forme :

$$
x m \theta\left(x^{-1}\right)=x^{\prime} m^{\prime} \theta\left(x^{-1}\right)
$$

ou encore : $y \widetilde{m} y^{-1}=\widetilde{m^{\prime}}$. On en déduit que : $\mathbf{P}_{\widetilde{m^{\prime}}}=y \mathbf{P}_{\widetilde{m}} y^{-1}$. Par hypothèse, $m, m^{\prime} \in$ $M_{\theta-c}^{+}$, donc $\mathbf{P}_{\widetilde{m}}=\mathbf{P}_{\widetilde{m}^{\prime}}=\mathbf{P}$. On en tire $: \mathbf{P}=y \mathbf{P} y^{-1}$. Mais cette égalité entraîne que $y \in P$, car $\mathbf{P}$ est un sous-groupe parabolique. Écrivons alors :

$$
y=\mu \nu, \quad \text { avec } \mu \in M, \nu \in N
$$

On a :

$$
u \cdot 1 \cdot n=u n=x=x^{\prime} y=x^{\prime} \mu \nu=u^{\prime} n^{\prime} \mu \nu=u^{\prime} \cdot \mu \cdot\left(\mu^{-1} n^{\prime} \mu \cdot \nu\right)
$$

Or, $u^{\prime} \in \bar{N}, \mu \in M, \mu^{-1} n^{\prime} \mu \in N, \nu \in N$, par unicité de la décomposition de la grosse cellule $\bar{N} M N$, on obtient :

$$
u=u^{\prime}, \quad \mu=1, \quad n=\mu^{-1} n^{\prime} \mu \nu=n^{\prime} \nu
$$


Il reste encore à démontrer que : $m=m^{\prime}$ et $n=n^{\prime}$. Puisque $u=u^{\prime}$, l'égalité 1.2 implique que : $n m \theta\left(n^{-1}\right)=n^{\prime} m^{\prime} \theta\left(n^{\prime-1}\right)$, ou encore :

$$
m \cdot m^{-1}\left(n^{\prime-1} n\right) m \cdot \theta\left(n^{-1} n^{\prime}\right)=m^{\prime}
$$

Puisque $m, m^{\prime} \in M, m^{-1}\left(n^{\prime-1} n\right) m, \theta\left(n^{-1} n^{\prime}\right) \in N$, cela implique que $m=m^{\prime}$ et $m^{-1}\left(n^{\prime-1} n\right) m \cdot \theta\left(n^{-1} n^{\prime}\right)=1$, que l'on peut écrire sous la forme : $\theta \circ \operatorname{Ad~} m^{-1}\left(n^{\prime-1} n\right)=$ $n^{\prime-1} n$. Par construction, $\theta \circ \mathrm{Ad} m^{-1}$ est strictement dilatant sur $N$ (c'est l'adjonction de $\left.\widetilde{m}^{-1}\right)$. L'égalité 1.3 implique donc $n^{\prime-1} n=1$, ce qui achève la preuve de la première assertion.

1.2.3. Preuve de l'assertion 2. - Commençons par le lemme suivant :

Lemme 1.4. - Soit $m \in M_{\theta-c}^{+}$fixé. Le jacobien du changement de variable

$$
\begin{aligned}
& N \longrightarrow N \\
& n \longmapsto m^{-1} n m \theta\left(n^{-1}\right)
\end{aligned}
$$

est égal à $D_{\theta}(m)$.

Démonstration. - Posons :

$$
\begin{aligned}
& \nu=\theta\left(m^{-1}\right) \theta(n) \theta(m) n^{-1} \\
& \nu^{\prime}=\theta(\nu)
\end{aligned}
$$

Le jacobien de l'application $\nu^{\prime} \mapsto \nu$ est égal à 1 , car $\theta$ est une involution. Montrons que le changement de variable $n \mapsto \nu^{\prime}$ a pour jacobien $d \nu^{\prime} / d n=D_{\theta}(m)^{-1}$. Le groupe $N$ étant unipotent, il existe une suite de sous-groupes distingués $N_{i}$ telle que :

$$
1=N_{0} \subset N_{1} \subset \cdots \subset N_{r}=N
$$

et pour tout $1 \leqslant i \leqslant r$, le quotient $N_{i} / N_{i-1}$ est unipotent et commutatif. En particulier $N_{i} / N_{i-1} \simeq \mathfrak{N}_{i} / \mathfrak{N}_{i-1}$, où $\mathfrak{N}_{i}$ désigne l'algèbre de Lie de $N_{i}$. On a :

$$
N \simeq \prod_{i} N_{i} / N_{i-1}
$$

L'application déduite de $n \mapsto \theta \circ \mathrm{Ad} m^{-1}(n) n^{-1}$ sur $N_{i} / N_{i-1}$ s'identifie à $n \mapsto \theta \circ$ $\operatorname{Ad} m^{-1}(n)-n$ sur $\mathfrak{N}_{i} / \mathfrak{N}_{i-1}$. Son jacobien est donc égal à

$$
\mid \operatorname{det}\left(\theta \circ \operatorname{Ad} m^{-1}-\mathrm{Id}\right)_{\left|\mathfrak{N}_{i} / \mathfrak{N}_{i-1}\right|}
$$

Le jacobien du changement de variable $n \mapsto \nu^{\prime}$ s'identifie au celui de

$$
\theta \circ \operatorname{Ad} m^{-1}-\mathrm{Id}: \prod_{i} N_{i} / N_{i-1} \simeq \prod_{i} \mathfrak{N}_{i} / \mathfrak{N}_{i-1} \longrightarrow \prod_{i} \mathfrak{N}_{i} / \mathfrak{N}_{i-1}
$$

qui est égal à $\prod_{i}\left|\operatorname{det}\left(\theta \circ \operatorname{Ad} m^{-1}-\mathrm{Id}\right)_{\mid \mathfrak{N}_{i} / \mathfrak{N}_{i-1}}\right|=\left|\operatorname{det}\left(\theta \circ \operatorname{Ad} m^{-1}-\mathrm{Id}\right)_{\mid \mathfrak{N}}\right|$. Mais comme $m \in M_{\theta-c}^{+}$, les valeurs propres de $\theta \circ \operatorname{Ad~} m^{-1}=(\operatorname{Ad} m \circ \theta)^{-1}$ dans $\mathfrak{N}$ sont de module $>1$. D'où : $\left|\operatorname{det}\left(\theta \circ \operatorname{Ad} m^{-1}-1\right)_{\mid \mathfrak{N}}\right|=\left|\operatorname{det}\left(\theta \circ \operatorname{Ad} m^{-1}\right)\right| \mathfrak{N} \mid=D_{\theta}(m)$. 
Considérons l'application :

$$
\begin{aligned}
\psi: \bar{N} \times M_{\theta-c}^{+} \times N & \longrightarrow G \\
(u, m, n) & \longmapsto g=u m n \theta\left(u^{-1}\right)
\end{aligned}
$$

Pour démontrer l'assertion 2, il suffit, moyennant le lemme précédent, de prouver que le jacobien $J\left(u_{0}, m_{0}, n_{0}\right)$ de $\psi$ est égal à 1 en tout triplet $\left(u_{0}, m_{0}, n_{0}\right)$. On peut se ramener au cas où $u_{0}=1$. En effet, au voisinage de $\left(u_{0}, m_{0}, n_{0}\right), \psi$ se décompose en le produit des trois applications :

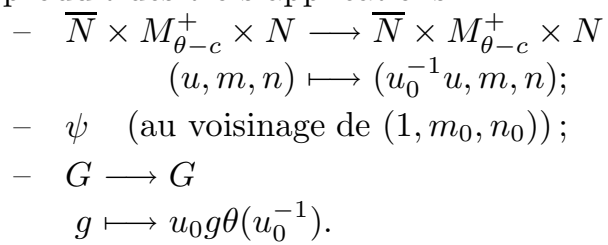

Les première et troisième applications ont visiblement 1 pour jacobien.

On est donc réduit à calculer la différentielle de $\psi$ en un triplet $(1, m, n)$. Identifions les espaces tangents $T_{m}(M), T_{1}(\bar{N}), T_{n}(N), T_{g}(G)$ aux algèbres de Lie $\mathfrak{M}, \overline{\mathfrak{N}}, \mathfrak{N}, \mathfrak{G}$ respectivement. Le jacobien $J(1, m, n)$ de $\psi$ est égal au module du déterminant de $d \psi_{(1, m, n)}$, vu comme un endomorphisme de $\overline{\mathfrak{N}} \oplus \mathfrak{M} \oplus \mathfrak{N}=\mathfrak{G}$. On a :

$$
\begin{aligned}
d \psi_{(1, m, n)}: \mathfrak{G}=\overline{\mathfrak{N}} \oplus \mathfrak{M} \oplus \mathfrak{N} \longrightarrow \mathfrak{G}=\overline{\mathfrak{N}} \oplus \mathfrak{M} \oplus \mathfrak{N} \\
(X, Y, Z) \longmapsto\left(\operatorname{Ad} n^{-1} m^{-1}-\theta\right)(X)+Y+Z
\end{aligned}
$$

Notons $p r_{\overline{\mathfrak{N}}}$ la projection de $\mathfrak{G}=\overline{\mathfrak{N}} \oplus \mathfrak{M} \oplus \mathfrak{N}$ sur $\overline{\mathfrak{N}}$, alors :

$$
J(1, m, n)=\left|\operatorname{det}\left(p r_{\overline{\mathfrak{N}}} \circ \operatorname{Ad} n^{-1} \circ \operatorname{Ad} m^{-1}-\theta\right)_{\mid \overline{\mathfrak{N}}}\right|
$$

Notons A la composante neutre du centre de M. Pour toute racine $\alpha$ de $A$ dans $\overline{\mathfrak{N}}$ fixons une base $\left\{X_{i}, i \in I(\alpha)\right\}$ de l'espace de racine correspondante. Ordonnons la base $\left\{X_{i}\right\}_{i \in \cup_{\alpha} I(\alpha)}$ de $\overline{\mathfrak{N}}$ ainsi obtenue, suivant un ordre décroissant sur la longueur de $\alpha$. Autrement dit, $X_{i}$ précède $X_{i^{\prime}}$ chaque fois que $i \in I(\alpha), i^{\prime} \in I\left(\alpha^{\prime}\right)$ et $\alpha$ est de plus grande longueur que $\alpha^{\prime}$. Pour $i \geqslant 1$ entier, notons $n_{i}$ le nombre de racines dans $\overline{\mathfrak{N}}$ qui sont de longueur $i$. Alors la matrice de $\operatorname{Ad} n$ dans la base $\left\{X_{i}, i \in I(\alpha)\right\}$ est de la forme:

$$
\left.\left(\begin{array}{cccc}
\operatorname{Id}_{n_{k}} & 0 & \cdots & 0 \\
* & \operatorname{Id}_{n_{k-1}} & \ddots & \vdots \\
* & * & \ddots & 0 \\
* & * & * & \operatorname{Id}_{n_{1}} \\
* & * & \cdots & *
\end{array}\right)\right\}_{\} \mathfrak{M}+\mathfrak{N}} \overline{\mathfrak{N}}
$$

Si $i \in I(\alpha)$ alors $\theta\left(X_{i}\right)$ est un vecteur propre de valeur propre $\theta(\alpha)$. Puisque $\alpha$ et $\theta(\alpha)$ sont de même longueur, la matrice de $\theta$ dans la base $\left\{X_{i}, i \in I(\alpha)\right\}$ est une matrice 
diagonale par blocs :

$$
\left(\begin{array}{cccc}
M_{k} & 0 & \cdots & 0 \\
0 & M_{k-1} & \ddots & \vdots \\
\vdots & \ddots & \ddots & 0 \\
0 & \cdots & 0 & M_{1}
\end{array}\right)
$$

où pour tout $i$, la matrice $M_{i}$ est de taille $n_{i} \times n_{i}$. Or, la matrice de $\operatorname{Ad}^{-1}$ dans cette base est diagonale, on en conclut que :

$$
\begin{aligned}
J(1, m, n) & =\left|\operatorname{det}\left(\operatorname{Ad} m^{-1}-\theta\right)_{\mid \overline{\mathfrak{M}}}\right| \\
& =\left|\operatorname{det}\left(\theta\left(\theta \circ \operatorname{Ad} m^{-1}-1\right)\right)_{\overline{\mathfrak{N}}}\right| \\
& =\left|\operatorname{det}\left(\theta \circ \operatorname{Ad} m^{-1}-1\right)_{\mid \overline{\mathfrak{N}}}\right| \\
& =1
\end{aligned}
$$

parce que $\theta \circ \operatorname{Ad~} m^{-1}$ contracte $\bar{N}$.

Le lemme s'ensuit, ainsi que la proposition.

\subsection{Formule de Clozel}

L'objet de ce paragraphe est de donner une formule analogue à celle obtenue par Clozel pour les traces compactes ( $c f$. [Clo, Prop. 2.1], et son corollaire).

Soit $\mathbf{P}=\mathbf{M N} \in \mathcal{P}^{\theta}$, on note $\delta_{P}$ la fonction module de $P$. Pour $f \in C_{c}^{\infty}(G)$ et $m \in M$, on pose :

$$
f^{\theta, P}(m)=\delta_{P}^{1 / 2}(m) \int_{N \times K} f\left(k m n \theta\left(k^{-1}\right)\right) d k d n
$$

Cette formule définit une fonction (localement constante, à support compact) sur $M$.

Soit $(\pi, V)$ une représentation lisse, irréductible, et $\theta$-stable de $G$. Soit $A \in$ $\operatorname{End}_{\mathbb{C}}(V)$, un opérateur qui entrelace $\pi$ et $\pi^{\theta}$, il est alors unique à un scalaire non nul près. L'opérateur $A$ se factorise en un opérateur, noté encore par $A$, qui entrelace le module de Jacquet normalisé $\pi_{N}$ et $\pi_{N}^{\theta}$. On peut donc parler des trace tordue, trace tordue compacte des modules de Jacquet pour les sous-groupes paraboliques $\theta$-stables.

Soit $\mathbf{P}=\mathbf{M N} \in \mathcal{P}^{\theta}$. Notons $\mathfrak{a}_{M}$ l'algèbre de Lie réelle de la composante neutre du centre de $\mathbf{M}$, et $H: M \rightarrow \mathfrak{a}_{M}$ l'application de Harish-Chandra; nous la définissons par : $|\chi(m)|=q^{-\langle\chi, H(m)\rangle}$. De façon similaire, notons $\mathfrak{a}_{M}^{\theta}$ le sous-espace des $\theta$-invariants de $\mathfrak{a}_{M}$. On définit $H^{\theta}: M \rightarrow \mathfrak{a}_{M}^{\theta}$ par la formule $H^{\theta}=H+\theta \circ H$. À $N$ est associé un système de racines simples dans $\mathfrak{a}_{M}$, noté $\Delta$. Notons $\tau_{P}^{G}, \widehat{\tau}_{P}^{G}$ les fonctions caractéristiques de la chambre de Weyl positive, chambre de Weyl obtuse positive respectivement, associée à $\Delta$. On note $a_{P}^{\theta}$ la dimension de $\mathfrak{a}_{M}^{\theta}$. On pose : $\chi_{N}^{\theta}=\tau_{P}^{G} \circ H^{\theta}, \widehat{\chi}_{N}^{\theta}=\widehat{\tau}_{P}^{G} \circ H^{\theta}$. 
ThÉorème 1.5. - Pour tout $f \in C_{c}^{\infty}(G)$, on a l'égalité :

$$
\operatorname{tr}_{\theta} \pi(f)=\sum_{\mathbf{P}=\mathbf{M N} \in \mathcal{P}^{\theta}} \operatorname{tr}_{\theta-c} \pi_{N}\left(\chi_{N}^{\theta} f^{\theta, P}\right)
$$

Démonstration. - Soit $g \in G$, il existe un $\theta$-conjugué de $g$, disons $g^{\prime}$ tel que $\mathbf{P}_{\widetilde{g}^{\prime}}$ soit $\theta$-stable, (voir les remarques qui précèdent la Prop. 7.4 dans $[\mathbf{R o 2}]$ ). On en déduit une décomposition de $G_{\theta-r e g}$ :

$$
G=\sqcup_{\mathbf{P}=\mathbf{M N} \in \mathcal{P}^{\theta}} \theta-\operatorname{Ad}(G)\left(M_{\theta-c}^{+}\right)
$$

Il suffit donc de démontrer que pour tout $\mathbf{P} \in \mathcal{P}^{\theta}$ fixé, on a l'égalité :

$$
\int_{\theta-\operatorname{Ad}(G)\left(M_{\theta-c}^{+}\right)} f(g) \operatorname{tr} \pi_{\theta}(g) d g=\operatorname{tr}_{\theta-c} \pi_{N}\left(f^{\theta, P}\right)
$$

En vertu de la proposition 1.1 on a l'égalité :

$$
\begin{array}{r}
\int_{\theta-a d(G)\left(M_{\theta-c}^{+}\right)} f(g) \operatorname{tr} \pi_{\theta}(g) d g=\int_{M_{\theta-c}^{+} \times N \times K} D_{\theta}(m) f\left(k n m \theta\left(n^{-1}\right) \theta\left(k^{-1}\right)\right) \\
\cdot \operatorname{tr}_{\theta} \pi(m) d k d n d m
\end{array}
$$

D’après Rogawski (cf. loc. cit.), si $m$ est $\theta$-régulier, alors :

$$
\operatorname{tr}_{\theta} \pi(m)=\delta_{P}^{1 / 2}(m) \operatorname{tr}_{\theta} \pi_{N}(m)
$$

C'est une version tordue d'un résultat de Casselman. Par définition de $f^{\theta, P}$, on a :

$$
\operatorname{tr}_{\theta-c} \pi\left(\chi_{N}^{\theta} f^{\theta, P}\right)=\int_{M_{\theta-c}^{+} \times N \times K} \delta_{P}^{1 / 2}(m) f\left(k m n \theta\left(k^{-1}\right)\right) \operatorname{tr}_{\theta} \pi_{N}(m) d k d n d m
$$

Par densité des éléments $\theta$-réguliers, il reste à vérifier l'identité :

$$
\int_{N \times K} f\left(k n m \theta\left(n^{-1}\right) \theta\left(k^{-1}\right)\right) d k d n=\int_{N \times K} D_{\theta}(m) f\left(k m n \theta\left(k^{-1}\right)\right) d k d n
$$

Mais cela vient du lemme 1.4.

On en déduit le résultat suivant, qui sera la clé de nos calculs ultérieurs :

THÉORÈme 1.6. - Soit $\pi$ une représentation $\theta$-stable de G. Alors, pour tout $f \in$ $C_{c}^{\infty}(G)$ on a l'identité :

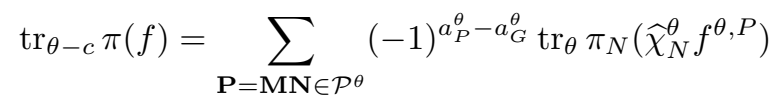

Démonstration. - C'est une version duale du théorème précédent. La démonstration se fait exactement par la même méthode que celle utilisée par Clozel pour prouver le corollaire à la proposition 2.1 dans [Clo]. 



\section{CHAPITRE 2}

\section{LES TRACES TORDUES COMPACTES SUR $G L(2 n+1)$}

\subsection{Les représentations considérées}

2.1.1. L'involution $\theta$. - Pour $N$ un entier positif, introduisons l'involution $\theta$ sur le groupe linéaire $\mathbf{G L}(N)$ de la façon suivante. Posons :

$$
w_{0}=\left(a_{i, j}\right)_{1 \leqslant i, j \leqslant N}
$$

où

$$
\begin{aligned}
a_{i, j} & =0 \quad \text { si } i+j \neq N+1 \\
a_{i, N+1-i} & =(-1)^{i-1}
\end{aligned}
$$

On définit alors $\theta$ par la formule :

$$
\theta(g)=w_{0}\left({ }^{t} g^{-1}\right) w_{0}^{-1}
$$

Le groupe $\mathbf{G L}(N)$, muni de $\theta$, satisfait les hypothèses décrites dans l'introduction.

2.1.2. Les représentations $\theta$-stables considérées. - Dans ce paragraphe, on considère un entier $n$ positif. Le groupe $\mathbf{G}$ sera le groupe linéaire $\mathbf{G L}(2 n+1)$. Nous introduisons quelques représentations $\theta$-stables de $G$.

Par définition, une partition est une suite d'entiers strictement positifs. Pour $\alpha=$ $\left(\alpha_{1}, \alpha_{2}, \ldots, \alpha_{r}\right)$ une partition, posons : $S(\alpha)=\alpha_{1}+\alpha_{2}+\cdots+\alpha_{r}$. Notons $\mathcal{A}^{\text {st }}(n)$ l'ensemble des triplets de partitions $\alpha^{+}=\left(\alpha_{1}^{+}, \alpha_{2}^{+}, \ldots, \alpha_{r}^{+}\right), \alpha^{-}=\left(\alpha_{1}^{-}, \alpha_{2}^{-}, \ldots, \alpha_{s}^{-}\right)$, $\beta=\left(\beta_{1}, \beta_{2}, \ldots, \beta_{t}\right)$ vérifiant les conditions suivantes :

$-S\left(\alpha^{+}\right)+S\left(\alpha^{-}\right)+2 S(\beta)=2 n+1 ;$

- Les suites $\left\{\alpha_{i}^{+}\right\}_{i=1, \ldots, r},\left\{\alpha_{j}^{-}\right\}_{j=1, \ldots, s}$ et $\left\{\beta_{k}\right\}_{k=1, \ldots t}$ sont strictement décroissantes. En plus, les suites $\left\{\alpha_{i}^{+}\right\}_{i=1, \ldots, r},\left\{\alpha_{j}^{-}\right\}_{j=1, \ldots, s}$ sont constituées de nombres impairs de multiplicité 1 ;

- Le nombre de termes de $\alpha^{-}$, c'est-à-dire $s$, est pair.

Soit $\left(\alpha^{+}, \alpha^{-}, \beta\right) \in \mathcal{A}^{\text {st }}(n)$. On note $\mathbf{R}=\mathbf{L V}$ le sous-groupe parabolique standard de $\mathbf{G}$ associé à la partition $\left(\beta_{1}, \ldots, \beta_{t}, \alpha_{1}^{+}, \ldots, \alpha_{r}^{+}, \alpha_{1}^{-}, \ldots, \alpha_{s}^{-}, \beta_{t}, \ldots, \beta_{1}\right)$. Notons $\xi$ le 
caractère quadratique non ramifié non trivial de $F^{\times}$. Pour tout $k$ entier nous écrivons $\mathrm{St}_{k}$ pour la représentation de Steinberg du groupe $\mathbf{G L}(k, F)$ et $\xi \mathrm{St}_{k}$ pour sa torsion par $\xi$. Définissons l'induite normalisée ( $c f$. Introduction) :

$$
\begin{aligned}
\pi & =\operatorname{St}_{\beta_{1}} \times \cdots \times \mathrm{St}_{\beta_{t}} \times \mathrm{St}_{\alpha_{1}^{+}} \times \cdots \times \mathrm{St}_{\alpha_{r}^{+}} \times \xi \mathrm{St}_{\alpha_{1}^{-}} \times \cdots \times \xi \mathrm{St}_{\alpha_{s}^{-}} \times \mathrm{St}_{\beta_{t}} \times \cdots \times \mathrm{St}_{\beta_{1}} \\
& =\mathrm{Ind}_{R}^{G}\left(\mathrm{St}_{\beta_{1}} \otimes \cdots \otimes \mathrm{St}_{\alpha_{1}^{+}} \otimes \cdots \otimes \mathrm{St}_{\alpha_{r}^{+}} \otimes \xi \mathrm{St}_{\alpha_{1}^{-}} \otimes \cdots \otimes \xi \mathrm{St}_{\alpha_{s}^{-}} \otimes \cdots \otimes \mathrm{St}_{\beta_{1}}\right)
\end{aligned}
$$

C'est une représentation de $G=\mathbf{G L}(2 n+1, F)$. La représentation $\pi$, qui dépend de $\left(\alpha^{+}, \alpha^{-}, \beta\right) \in \mathcal{A}^{\text {st }}(n)$, est lisse. En plus, d'après la classification de Zelevinsky, elle est irréductible et $\theta$-stable.

Soit $K=\mathbf{G}\left(\mathcal{O}_{F}\right)$, notons $\mathcal{H}(G)$ l'ensemble des fonctions sur $G$, biinvariantes par $K$ et à support compact (muni du produit de convolution relatif à la mesure de Haar qui donne 1 pour volume de $K$ ). C'est l'algèbre de Hecke sphérique usuelle de $G$. Pour $\mathbf{P}=\mathbf{M N}$ un sous-groupe parabolique standard de $\mathbf{G}$, on définit l'algèbre de Hecke sphérique de $M$, notée $\mathcal{H}(M)$, comme l'ensemble des fonctions sur $M$, biinvariantes par $K_{M}=K \cap M$ et à support compact.

Pour $\left(\alpha^{+}, \alpha^{-}, \beta\right) \in \mathcal{A}^{\text {st }}(n)$, soit $\pi$ la représentation de $G$ définie comme ci-dessus. Nous nous proposons de calculer explicitement la trace tordue compacte $\operatorname{tr}_{\theta-c} \pi$ sur l'algèbre de Hecke sphérique de $G$.

2.1.3. Notations des caractères non ramifiés, le caractère $\eta$. - Pour tout $k$ entier positif, les caractères non ramifiés du tore diagonal du groupe $\mathbf{G L}(k, F)$ sont de la forme :

$$
\chi\left(t_{1}, \ldots, t_{k}\right)=\left|t_{1}\right|^{\chi_{1}} \cdots\left|t_{k}\right|^{\chi_{k}}
$$

où $\chi_{1}, \ldots, \chi_{k}$ sont des nombres complexes (modulo $2 i \pi / \log q$ ). Dans la suite, pour un tel $\chi$, nous écrivons simplement :

$$
\chi=\left(\chi_{1}, \ldots, \chi_{k}\right)
$$

On dira que $\chi$ est symétrique si pour tout $i=1, \cdots, k$ on a l'égalité :

$$
\chi_{i}=-\chi_{k+1-i} \bmod \frac{2 i \pi}{\log q}
$$

Posons $\varepsilon=i \pi / \log q$. Soit $\left(\alpha^{+}, \alpha^{-}, \beta\right) \in \mathcal{A}^{\text {st }}(n)$, on définit le caractère non ramifié $\eta$ du tore diagonal par la formule :

$$
\begin{array}{r}
\eta=\left(\frac{\beta_{1}-1}{2}, \ldots, \frac{1-\beta_{1}}{2}, \ldots, \frac{\beta_{t}-1}{2}, \ldots, \frac{1-\beta_{t}}{2}, \frac{\alpha_{1}^{+}-1}{2}, \ldots, \frac{1-\alpha_{1}^{+}}{2}, \ldots, \frac{\alpha_{r}^{+}-1}{2}, \ldots,\right. \\
\frac{1-\alpha_{r}^{+}}{2}, \frac{\alpha_{1}^{-}-1}{2}+\varepsilon, \ldots, \frac{1-\alpha_{1}^{-}}{2}+\varepsilon, \ldots, \frac{\alpha_{s}^{-}-1}{2}+\varepsilon, \ldots, \frac{1-\alpha_{s}^{-}}{2}+\varepsilon, \frac{\beta_{t}-1}{2}, \\
\left.\ldots, \frac{1-\beta_{t}}{2}, \ldots, \frac{\beta_{1}-1}{2}, \ldots, \frac{1-\beta_{1}}{2}\right)
\end{array}
$$

Ce n'est autre que le module de Jacquet relatif au Borel de la représentation

$$
\mathrm{St}_{\beta_{1}} \otimes \cdots \otimes \mathrm{St}_{\alpha_{1}^{+}} \otimes \cdots \otimes \mathrm{St}_{\alpha_{r}^{+}} \otimes \xi \mathrm{St}_{\alpha_{1}^{-}} \otimes \cdots \otimes \xi \mathrm{St}_{\alpha_{s}^{-}} \otimes \cdots \otimes \mathrm{St}_{\beta_{1}}
$$


du Lévi $L$ de $R$. Remarquons que la représentation $\pi$ associée au triplet $\left(\alpha^{+}, \alpha^{-}, \beta\right)$

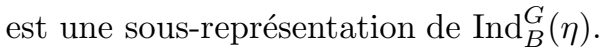

\subsection{Filtration de Bernstein-Zelevinsky}

Soient $\mathbf{P}=\mathbf{M}_{\mathbf{P}} \mathbf{N}_{\mathbf{P}}, \mathbf{Q}=\mathbf{M}_{\mathbf{Q}} \mathbf{N}_{\mathbf{Q}}$ deux sous-groupes paraboliques standard de $\mathbf{G}$. Soit $\rho$ une représentation lisse de $M_{P}$, posons $\pi=\operatorname{Ind}_{P}^{G}(\rho)$ et notons $V$ son espace. Il s'agit de donner une filtration du module de Jacquet $\pi_{N_{Q}}$. Fixons d'abord quelques notations. L'ensemble des doubles classes $W^{M_{Q}} \backslash W / W^{M_{P}}$ a un système de représentants bien particulier que l'on va noter par $\left[W^{M_{Q}} \backslash W / W^{M_{P}}\right]$. Pour qu'un élément $w \in W$ appartienne à $\left[W^{M_{Q}} \backslash W / W^{M_{P}}\right]$, il faut et il suffit qu'il soit de longueur minimale dans $W^{M_{Q}} w$ et dans $w W^{M_{P}}$. On va noter ce système par $\left[W^{M_{Q}} \backslash W\right]$ lorsque $\mathbf{P}=\mathbf{B}$, et $\left[W / W^{M_{P}}\right]$ lorsque $\mathbf{Q}=\mathbf{B}$. Dans la suite, on appelle ce système système de représentants distingués.

Posons : $d(w)=\operatorname{dim} \mathbf{P} w^{-1} \mathbf{Q}-\operatorname{dim} \mathbf{P Q}$. Pour tout entier positif $r$, posons :

$$
X_{r}=\cup_{d(w)<r} P w^{-1} Q
$$

Les ensembles $X_{r}$ sont fermés dans $G$ pour la topologie de Zariski, a fortiori pour la topologie $p$-adique. Notons $Y_{r}$ le complémentaire de $X_{r}$ dans $G$ et posons :

$$
V_{r}=\left\{f \in V ; f \text { à support dans } Y_{r}\right\}
$$

Les $V_{r}$ forment une filtration décroissante de $V$. En plus, cette filtration est $Q$-stable. On montre que, pour tout $r$, le quotient $V_{r} / V_{r+1}$ s'identifie à l'espace des fonctions $f$ localement constantes sur $X_{r+1}$, s'annulant sur $X_{r}$ et vérifiant la condition :

$$
f(m n g)=\delta_{P}^{1 / 2}(m) \rho(m) f(g) \quad \text { pour tous } m \in M_{P}, n \in N_{P}, g \in X_{r+1}
$$

D'autre part, $X_{r+1} \backslash X_{r}=\sqcup_{d(w)=r} P w^{-1} Q$, et chaque $P w^{-1} Q$ avec $d(w)=r$ est ouvert dans $X_{r+1}$. Soit $J_{w}$ l'ensemble des fonctions $f$ sur $P w^{-1} Q$ (à valeurs toujours dans l'espace de $\rho$ ), s'annulant en dehors d'un ensemble de la forme $P C$ avec $C$ compact, et vérifiant la condition ci-dessus. On a donc :

$$
V_{r} / V_{r+1} \simeq \oplus_{d(w)=r} J_{w}
$$

Le groupe $M_{P} \cap w^{-1} Q w$ est un sous-groupe parabolique de $M_{P}$ de radical unipotent $M_{P} \cap w^{-1} N_{Q} w$ et de facteur de Lévi $M_{P} \cap w^{-1} M_{Q} w$. Il en est de même pour $M_{Q} \cap w P w^{-1}, M_{Q} \cap w N_{P} w^{-1}, M_{Q} \cap w M_{P} w^{-1}$. L'espace $\left(J_{w}\right)_{N_{Q}}$, qui est une

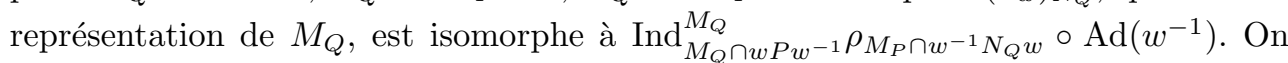
notera cette représentation par $F_{w}(\pi)$.

En résumé, on a montré qu'il existe une filtration de $\pi_{N_{Q}}$, qui est indexée par $\left[W^{M_{Q}} \backslash W / W^{M_{P}}\right]$, telle que la composante correspondante à $w \in\left[W^{M_{Q}} \backslash W / W^{M_{P}}\right]$ soit isomorphe à l'induite $\operatorname{Ind}_{M_{Q} \cap w P w^{-1}}^{M_{Q}} \rho_{M_{P} \cap w^{-1} N_{Q} w} \circ \operatorname{Ad}\left(w^{-1}\right)$. Nous pouvons alors 
écrire le gradué associé à la filtration de Bernstein-Zelevinsky par :

$$
J H\left(\pi_{N_{Q}}\right)=\sum_{w \in\left[W^{\left.M_{Q} \backslash W / W^{M_{P}}\right]}\right.} F_{w}(\pi)
$$

\subsection{Normalisation des opérateurs d'entrelacement}

Dans ce paragraphe on montre qu'il existe une droite $D$ bien définie dans $V$, l'espace de la représentation $\pi$ en question, qui est invariante par $A$, l'opérateur d'entrelacement entre $\pi$ et $\pi^{\theta}$. On normalise $A$ de sorte qu'il fixe $D$.

2.3.1. Algèbre de Hecke-Iwahori de GL $(2 n+1, F)$. — Soit $I$ le groupe d'Iwahori usuel de $G$. Notons $\mathcal{H}(G, I)$ l'algèbre de Hecke-Iwahori correspondante. C'est l'ensemble des fonctions complexes sur $G$, biinvariantes par $I$, à support compact, et muni du produit de convolution relatif à la mesure de Haar pour laquelle mes $(I)=1$. Pour $i=1, \ldots, 2 n$, notons $s_{i}$ la symétrie élémentaire qui échange $i$ et $i+1$. L'algèbre $\mathcal{H}(G, I)$ est alors engendrée par des éléments $T_{s_{i}}, i=1, \ldots 2 n$, et $X_{i}^{ \pm 1}, i=1, \ldots 2 n+1$. Pour tous $i, j$, on a les relations suivantes :

$$
\begin{aligned}
\left(T_{s_{i}}+1\right)\left(T_{s_{i}}-q\right) & =0 \\
T_{s_{i+1}} T_{s_{i}} T_{s_{i+1}} & =T_{s_{i}} T_{s_{i+1}} T_{s_{i}} \\
X_{i} X_{j} & =X_{j} X_{i} \\
X_{i} T_{s_{j}} & =T_{s_{j}} X_{i} \quad \text { si } i \neq j, j+1 \\
X_{i} T_{s_{i}} & =T_{s_{i}} X_{i+1}-(q-1) X_{i+1} \\
X_{i+1} T_{s_{i}} & =T_{s_{i}} X_{i}+(q-1) X_{i+1}
\end{aligned}
$$

Ces relations forment un système complet de relations (cf. [Ro1, Paragraphe 1]). Soit $w \in W$ et soit $w=s_{i} \cdots s_{j}$ une décomposition réduite de $w$, l'élément $T_{w}=T_{s_{i}} \cdots T_{s_{j}}$ est alors bien défini. Concrètement $T_{w}$ est la fonction caractéristique de l'ensemble $I w I$.

On peut décrire les $X_{i}$ comme suit. Pour $i=0, \ldots, 2 n+1$, soit $m_{i}$ la matrice diagonale de taille $2 n+1$ dont les $i$ premiers termes sont égaux à 1 , et les $2 n+1-i$ derniers à $\varpi$. Soit $C\left(m_{i}\right)$ la fonction caractéristique de l'ensemble $I m_{i} I$. Les éléments $C\left(m_{i}\right)$ sont alors inversibles dans $\mathcal{H}(G, I)$ et on a l'égalité :

$$
X_{i}=q^{n+1-i} C\left(m_{i-1}\right) C\left(m_{i}\right)^{-1}
$$

Notons $\ell: W \rightarrow \mathbb{N}$ la fonction longueur usuelle sur $W$. Notons aussi $<$ l'ordre partiel de Bruhat sur $W$. On en déduit les relations suivantes :

$$
\begin{aligned}
& T_{w w^{\prime}}=T_{w} T_{w^{\prime}} \quad \text { si } \ell\left(w w^{\prime}\right)=\ell(w)+\ell\left(w^{\prime}\right) \\
& X_{i} T_{w}=T_{w} X_{w^{-1}(i)}+\sum_{w^{\prime}<w} T_{w^{\prime}} a_{w^{\prime}}
\end{aligned}
$$

où les $a_{w^{\prime}}$ sont des polynômes en les variables $X_{i}$. 
Les éléments $T_{w}, w \in W$ forment une base d'une sous-algèbre de $\mathcal{H}(G, I)$ que l'on va noter $\operatorname{par} \mathcal{H}(G, I)_{W}$.

2.3.2. La droite $D$. - On note $D$ la droite engendrée par l'élément $\delta$ défini par la formule :

$$
\delta=\sum_{w \in W}(-q)^{-\ell(w)} T_{w}
$$

Lemme 2.3. - Pour tout $w \in W$ on a l'égalité :

$$
T_{w} \delta=(-1)^{\ell(w)} \delta
$$

Démonstration. - Il suffit de traiter le cas où $w=s_{i}$ une symétrie élémentaire. Écrivons :

$$
\delta=\left(1-q^{-1} T_{s_{i}}\right) \delta^{\prime}
$$

où

$$
\delta^{\prime}=\sum_{\substack{w \in W \\ \ell\left(s_{i} w\right)=\ell(w)+1}}(-q)^{-\ell(w)} T_{w}
$$

L'égalité cherchée vient alors de la relation $\left(T_{s_{i}}+1\right)\left(T_{s_{i}}-q\right)=0$.

Le caractère de $\mathcal{H}(G, I)_{W}, T_{w} \mapsto(-1)^{\ell(w)}$ s'appelle caractère de Steinberg, on le note $\mathrm{St}_{2 n+1, W}$. D'après le lemme précédent, il intervient dans la représentation régulière gauche de $\mathcal{H}(G, I)_{W}$ et est porté par la droite $D$. En plus, on sait que sa multiplicité est 1.

2.3.3. Algèbre de Hecke sphérique. - Rappelons que l'algèbre $\mathcal{H}(G)$ des fonctions biinvariantes par le compact maximal $K$, à support compact est isomorphe à $\mathbb{C}\left[X_{1}^{ \pm 1}, \ldots, X_{2 n+1}^{ \pm 1}\right]$ via l'isomorphisme de Satake, noté $S$. Posons :

$$
\gamma=\sum_{w \in W} T_{w}=\mathbf{1}_{K}
$$

On a l'égalité :

$$
[K: I]=\sum_{w \in W} q^{\ell(w)}
$$

L'algèbre $\mathcal{H}(G)$ s'identifie à un sous-ensemble de $\mathcal{H}(G, I)$ par l'application suivante :

$$
f \longmapsto[K: I]^{-1} \gamma \cdot S(f)\left(X_{1}, \ldots, X_{2 n+1}\right)=[K: I]^{-1} S(f)\left(X_{1}, \ldots, X_{2 n+1}\right) \cdot \gamma
$$

Cette application respecte l'addition et la multiplication mais ne conserve pas les unités. 
2.3.4. Automorphisme $\theta$ de $\mathcal{H}(G, I)$. - Pour toute fonction $f$ sur $G$ on a défini la fonction $f^{\theta}$ par la formule : $f^{\theta}(g)=f(\theta(g))$. L'application $f \mapsto f^{\theta}$ induit un automorphisme d'algèbres sur $\mathcal{H}(G, I)$ que l'on va encore noter par $\theta$. Les égalités suivantes viennent alors des descriptions ci-dessus des éléments de $\mathcal{H}(G, I)$. Pour tous $w \in W, i=1, \ldots, 2 n+1$ :

$$
\begin{aligned}
& \theta\left(T_{w}\right)=T_{\theta(w)} \\
& \theta\left(X_{i}\right)=X_{2 n+2-i}^{-1}
\end{aligned}
$$

2.3.5. Les séries principales non ramifiées et leurs invariants par $I$. - Soit $\chi=\left(\chi_{1}, \ldots, \chi_{2 n+1}\right)$ un caractère non ramifié du tore diagonal $T$ ( $c f .2 .1 .3$ pour la notation). Considérons $\left(\operatorname{Ind}_{B}^{G}(\chi), \mathcal{I}(\chi)\right)$, l'induite parabolique normalisée de $\chi$. L'espace $\mathcal{I}(\chi)^{I}$ des invariants par $I$ est une représentation de $\mathcal{H}(G, I)$ dont nous rappelons la description en suivant [Ro1].

À un élément $T \in \mathcal{H}(G, I)_{W}$, on associe l'unique fonction $\phi(T) \in \mathcal{I}(\chi)$ telle que pour tout $k \in K, \phi(T)(k)=T\left(k^{-1}\right)$. L'application $\phi$ définit un isomorphisme de $\mathcal{H}(G, I)_{W}$-modules entre $\mathcal{H}(G, I)_{W}$ (qui est muni de la représentation régulière gauche) et $\mathcal{I}(\chi)^{I}$. Nous allons construire une action de $\mathcal{H}(G, I)$ sur $\mathcal{H}(G, I)_{W}$ étendant la représentation régulière gauche, de sorte que $\phi$ devienne un isomorphisme de $\mathcal{H}(G, I)$-modules.

Soit $\mathcal{H}(G, I)[\chi]$ l'idéal à gauche engendré par les éléments $X_{i}-q^{-\chi_{i}}, i=1, \ldots, 2 n+1$. L'espace $\mathcal{H}(G, I) / \mathcal{H}(G, I)[\chi]$ est muni de façon naturelle d'une action de $\mathcal{H}(G, I)$. On a un diagramme commutatif :

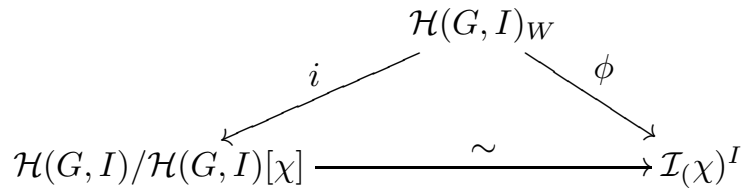

où $i$ est le composé de l'injection canonique $\mathcal{H}(G, I)_{W} \hookrightarrow \mathcal{H}(G, I)$ et de la projection canonique de $\mathcal{H}(G, I)$ sur $\mathcal{H}(G, I) / \mathcal{H}(G, I)[\chi]$. Les flèches obliques sont des isomorphismes de $\mathcal{H}(G, I)_{W}$-modules et celle horizontale de $\mathcal{H}(G, I)$-modules.

Remarquons au passage que si $\mathbf{P}=\mathbf{M N}$ est un sous-groupe parabolique standard de $\mathbf{G}$, avec des notations usuelles, on a un isomorphisme : $\mathcal{I}(\chi)^{I} \simeq \mathcal{I}(\chi)_{N}^{I_{M}}$. Si on identifie $\mathcal{I}(\chi)^{I}$ à $\mathcal{H}(G, I)_{W}$ par l'application $\phi$, alors la représentation de $\mathcal{H}\left(M, I_{M}\right)$ sur $\mathcal{I}(\chi)_{N}^{I_{M}}$ s'obtient de celle de $\mathcal{H}(G, I)$ sur $\mathcal{H}(G, I)_{W}$ par restriction à $\mathcal{H}\left(M, I_{M}\right)$.

\subsubsection{Les représentations auxquelles on s'intéresse et leurs invariants par $I$}

Par construction, une représentation $(\pi, V)$ définie comme en 2.1 à partir d'un triplet $\left(\alpha^{+}, \alpha^{-}, \beta\right)$, est une sous-représentation d'une induite à partir d'un caractère non ramifié $\eta$ bien déterminé de $T$ ( $c f .2 .1 .3$ ). Toujours par construction, $\pi$ est définie comme une induite normalisée à partir d'une représentation d'un Lévi standard $\mathbf{L}$ 
dont le groupe de Weyl est noté $W^{L}$. Posons :

$$
\delta^{L}=\sum_{w \in W^{L}}(-q)^{-\ell(w)} T_{w}, \quad \gamma^{L}=\sum_{w \in W^{L}} T_{w}
$$

On a : $(\pi, V) \hookrightarrow\left(\operatorname{Ind}_{B}^{G}(\eta), \mathcal{I}(\eta)\right)$. L'espace $\mathcal{I}(\eta)^{I}$ peut être identifié à $\mathcal{H}(G, I)_{W}$ via $\phi$ comme on a vu. Décrivons l'espace $V^{I}$.

Lemme 2.4. - L'image réciproque de $V^{I}$ par $\phi$ est égale à $\mathcal{H}(G, I)_{W} \cdot \delta^{L}$. C'est aussi le sous-espace de $\mathcal{H}(G, I)_{W}$ de base $\left\{T_{w} \delta^{L}\right\}_{w \in\left[W / W^{L}\right]}$.

Démonstration. - Dans [Wa1, Paragraphe 5], Waldspurger définit les éléments $\tau_{w}, w \in W$ de Lusztig. La multiplication à droite par ces éléments définit des opérateurs d'entrelacement. En particulier, lorsque $w=w^{L}$, l'élément de plus grande longueur de $W^{L}$, l'image de l'opérateur d'entrelacement correspondant est exactement $\phi^{-1}\left(V^{I}\right)$. D'autre part, par un calcul élémentaire, on montre que $\tau_{w^{L}}$ est un multiple non nul de $\delta^{L}$. Donc, $\phi^{-1}\left(V^{I}\right)=\mathcal{H}(G, I)_{W} \cdot \delta^{L}$. Soit $w \in W$, on peut écrire $w=w_{1} w_{0}$ avec $w_{0} \in W^{L}, w_{1} \in\left[W / W^{L}\right]$. On a alors $\ell(w)=\ell\left(w_{0}\right)+\ell\left(w_{1}\right)$, et donc $T_{w}=T_{w_{1}} T_{w_{0}}$. On en déduit :

$$
T_{w} \delta^{L}=T_{w_{1}} T_{w_{0}} \delta^{L}=(-1)^{\ell\left(w_{0}\right)} T_{w_{1}} \delta^{L}
$$

L'espace $\phi^{-1}\left(V^{I}\right)$ est donc engendré par les $\left\{T_{w} \delta^{L}\right\}_{w \in\left[W / W^{L}\right]}$. Pour montrer que cette famille est libre, il suffit de remarquer que pour $w \in\left[W / W^{L}\right]$,

$$
T_{w} \delta^{L}=\sum_{w^{\prime} \in W^{L}}(-q)^{-\ell\left(w^{\prime}\right)} T_{w w^{\prime}} .
$$

Corollaire 2.5. - La droite $D$ définie en 2.3.2 est contenue dans $\phi^{-1}\left(V^{I}\right)$.

En effet, il suffit d'écrire :

$$
\delta=\left(\sum_{w \in\left[W / W^{L}\right]}(-q)^{-\ell(w)} T_{w}\right) \delta^{L}
$$

2.3.7. Normalisation de $A: \pi \simeq \pi^{\theta}$. — Puisque $\theta(I)=I$, l'opérateur $A$ conserve $V^{I}$. La restriction de $A$ à $V^{I}$ entrelace la représentation naturelle de $\mathcal{H}(G, I)$ et sa torsion par l'automorphisme $\theta$ déjà introduit. Identifions $V^{I}$ à un sous-espace de $\mathcal{H}(G, I)$ par l'application $\phi$. Par définition, la droite $D$ porte le caractère de Steinberg de $\mathcal{H}(G, I)_{W}$. On en déduit que $A(D)$ porte aussi le caractère de Steinberg parce que ce caractère est stable par $\theta$. Comme la multiplicité de la Steinberg est 1 , la droite $D$ est stable par $A$. On normalise $A$ de sorte qu'il fixe $D$. 
2.3.8. Les espaces $V_{N}^{K_{M}}$. — Profitons de l'occasion pour déterminer l'espace $V_{N}^{K_{M}}$, lorsque $\mathbf{P}=\mathbf{M N}$ est un sous-groupe parabolique standard. On sait que l'espace $V_{N}^{I_{M}}$ est isomorphe à $V^{I}$, et si on identifie $V^{I}$ à $\mathcal{H}(G, I)_{W}$ via $\phi$, la représentation de $\mathcal{H}\left(M, I_{M}\right)$ sur $\mathcal{I}(\eta)_{N}^{I_{M}}$ s'obtient de celle de $\mathcal{H}(G, I)$ sur $\mathcal{H}(G, I)_{W}$ par restriction. On a vu comment l'algèbre de Hecke $\mathcal{H}(M)$ se plonge dans $\mathcal{H}\left(M, I_{M}\right)$. Remarquons que l'élément $\gamma^{M}$ (ou plutôt, $\phi\left(\gamma^{M}\right)$ ) est un multiple de la fonction caractéristique de $K_{M}$. Par ailleurs, on a : $\phi^{-1}\left(V^{I}\right)=\mathcal{H}(G, I)_{W} \cdot \delta^{L}$. Il en résulte que :

$$
\phi^{-1}\left(V_{N}^{K_{M}}\right)=\gamma^{M} \cdot \mathcal{H}(G, I)_{W} \cdot \delta^{L}
$$

On sait (cf. par exemple [Car, Prop. 2.7.5]) que tout $w \in W$ peut s'écrire (de façon non unique) sous la forme $w=w_{1} w_{0} w_{2}$ où $w_{1} \in W^{M}, w_{0} \in\left[W^{M} \backslash W / W^{L}\right], w_{2} \in W^{L}$ et telle que $\ell(w)=\ell\left(w_{1}\right)+\ell\left(w_{0}\right)+\ell\left(w_{2}\right)$. Pour une telle décomposition, on a $T_{w}=$ $T_{w_{1}} T_{w_{0}} T_{w_{2}}$. Par induction on peut démontrer l'égalité $: \gamma^{M} T_{w_{1}}=q^{\ell\left(w_{1}\right)} \gamma^{M}$. Mais comme $T_{w_{2}} \delta^{L}=(-1)^{\ell\left(w_{2}\right)} \delta^{L}$, on obtient :

$$
\gamma^{M} T_{w} \delta^{L}=q^{\ell\left(w_{1}\right)}(-1)^{\ell\left(w_{2}\right)} \gamma^{M} T_{w_{0}} \delta^{L} .
$$

Il en résulte que $\phi^{-1}\left(V_{N}^{K_{M}}\right)$ est engendré par les éléments $\gamma^{M} T_{w} \delta^{L}, w \in\left[W^{M} \backslash W / W^{L}\right]$.

On définit :

$$
\left[W^{M} \backslash W / W^{L}\right]^{0}=\left\{w \in\left[W^{M} \backslash W / W^{L}\right] ; W^{M} \cap w W^{L} w^{-1}=\{1\}\right\}
$$

Lemme 2.6. - Soit $w \in\left[W^{M} \backslash W / W^{L}\right]$. Si $w \notin\left[W^{M} \backslash W / W^{L}\right]^{0}$, alors $\gamma^{M} \cdot T_{w} \cdot \delta^{L}=0$. $L^{\prime}$ 'espace $\phi^{-1}\left(V_{N}^{K_{M}}\right)$ a pour base l'ensemble des éléments $\gamma^{M} \cdot T_{w} \cdot \delta^{L}, w \in\left[W^{M} \backslash W / W^{L}\right]^{0}$.

Démonstration. - Si $w \notin\left[W^{M} \backslash W / W^{L}\right]^{0}$, par définition, le sous-groupe de Weyl $W^{M} \cap w W^{L} w^{-1}$ n'est pas réduit à 1 . Puisque $w \in\left[W^{M} \backslash W / W^{L}\right]$, on peut alors trouver une symétrie élémentaire $s_{i} \in W^{L}$ de sorte que $w s_{i} w^{-1} \in W^{M}$ soit aussi une symétrie élémentaire, disons $s_{j}$. Écrivons

$$
\delta^{L}=\left(1-q^{-1} T_{s_{i}}\right) \delta^{L}, \quad \gamma^{M}=\gamma^{M}\left(1+T_{s_{j}}\right)
$$

où

$$
\left.\delta^{L}=\sum_{\substack{w \in W^{L} \\ \ell\left(s_{i} w\right)=\ell(w)+1}}(-q)^{-\ell(w}\right) T_{w}, \quad \gamma^{M}=\sum_{\substack{w \in W^{M} \\ \ell\left(w s_{j}\right)=\ell(w)+1}} T_{w}
$$

On a :

$$
\begin{aligned}
\left(1+T_{s_{j}}\right) T_{w}\left(1-q^{-1} T_{s_{i}}\right) & =\left(1+T_{s_{j}}\right)\left(T_{w}-q^{-1} T_{w s_{i}}\right) \\
& =\left(1+T_{s_{j}}\right)\left(T_{w}-q^{-1} T w s_{i} w^{-1} . w\right) \\
& =\left(1+T_{s_{j}}\right)\left(T_{w}-q^{-1} T_{s_{j}} T_{w}\right) \\
& =\left(1+T_{s_{j}}\right)\left(1-q^{-1} T_{s_{j}}\right) T_{w} \\
& =0
\end{aligned}
$$

On en déduit que l'espace $\phi^{-1}\left(V_{N}^{K_{M}}\right)$ est engendré par les éléments $\gamma^{M} T_{w} \delta^{L}$, $w \in\left[W^{M} \backslash W / W^{L}\right]^{0}$. Il reste à montrer que ces éléments sont linéairement indépendants. Soit $w \in\left[W^{M} \backslash W / W^{L}\right]^{0}$, d'après un résultat de Howlett (cf. [Car, 
Prop. 2.7.5]), pour tous $w_{1} \in W^{M}, w_{2} \in W^{L}$, on a $\ell\left(w_{1} w w_{2}\right)=\ell\left(w_{1}\right)+\ell(w)+\ell\left(w_{2}\right)$. Par suite,

$$
\gamma^{M} T_{w} \delta^{L}=\sum_{w_{1} \in W^{M}, w_{2} \in W_{L}} q^{\ell\left(w_{1}\right)}(-1)^{\ell\left(w_{2}\right)} T_{w_{1} w w_{2}}
$$

Les éléments $T_{w_{1} w w_{2}}$ dans la formule précédente sont tous distincts. L'indépendance linéaire des $\gamma^{M} T_{w} \delta^{L}$ en résulte, ainsi que le lemme.

Remarque 2.7. - Cette description de $V_{N}^{M_{K}}$ permet également de faire des calculs dans les cas $\pi$ symétriques (i.e. dans les cas où $A$ est de la forme $f \mapsto f^{\theta}$ ) sans faire appel à la filtration de Bernstein-Zelevinsky.

2.3.9. Rappels sur les opérateurs d'entrelacement. — Soit $\chi$ un caractère non ramifié de $T$. Soit $\operatorname{Ind}_{B}^{G}(\chi)$ l'induite de $\chi$, et notons $I(\chi)$ son espace. Notons $J$ l'espace des fonctions sur le compact maximal usuel $K$, invariantes à droite par un sous-groupe ouvert, et invariantes à gauche par $K \cap B$. Par restriction à $K$, on peut alors identifier $I(\chi)$ à $J$, c'est ce que l'on fera. Remarquons que $J$ ne dépend pas du caractère $\chi$ choisi.

Notons $\overline{\mathbf{U}}$ le radical unipotent du sous-groupe parabolique opposé à $\mathbf{P}$. Soit $w \in W$, posons $U_{w}=U \cap w \bar{U} w^{-1}$. Soient $f \in \mathrm{J}$, définissons l'opérateur $A(w)$ par la formule :

$$
A(w) f(g)=\int_{U_{w}} f\left(w^{-1} u g\right) d u
$$

quand cela converge. C'est l'intégrale d'entrelacement bien connue. Écrivons $\chi=$ $\left(\chi_{1}, \ldots, \chi_{n}\right)$, posons :

$$
c_{w}(\chi)=\prod_{\substack{i<j \\ w(i)>w(j)}} \frac{-1+q^{\chi_{i}-\chi_{j}}}{1-q^{-1+\chi_{i}-\chi_{j}}} \text { et } N(w, \chi)=c_{w}(\chi) A(w)
$$

La restriction de cet opérateur à $I(\chi)^{I}$ est égale à $A_{w^{-1}}$, l'opérateur défini par Waldspurger dans $[\mathbf{W a 1}]$, multiplié par $(-1)^{\ell(w)}$. Cette normalisation n'est pas la normalisation la plus usuelle. En effet, la normalisation usuelle de $A(w)$ fixe la droite sphérique dans $I(\chi)$ alors que dans notre normalisation, c'est la droite $D$ définie en 2.3.1 qui est fixe, d'après la formule [Wa1, V.4 (3)].

Pour $w=s_{i}$ la symétrie simple qui échange $i$ et $i+1$, l'opérateur $N\left(s_{i}, \chi\right)$ ne dépend que de $\chi_{i}-\chi_{i+1}$. On écrira $N\left(s_{i}, \chi_{i}-\chi_{i+1}\right)$ au lieu de $N\left(s_{i}, \chi\right)$. Rappelons quelques propriétés des $N(w, \chi)$ :

- Pôles : les opérateurs $N(w, \chi)$ sont méromorphes en $\chi_{i}$. Les pôles de $N(w, \chi)$ sont simples et sont les pôles de $c_{w}(\chi)$. Les pôles de $N\left(s_{i}, \chi\right)$ sont en $\chi_{i}=1+\chi_{i+1}$;

- Entrelacement : lors qu'il est défini, $N(w, \chi)$ entrelace les représentations $\operatorname{Ind}_{B}^{G}(\chi)$ et $\operatorname{Ind}_{B}^{G}(w \chi)$;

- Formule du produit : pour $w, w^{\prime} \in W, N\left(w^{\prime}, w \chi\right) N(w, \chi)=N\left(w^{\prime} w, \chi\right)$;

- Valeur en 0 : on a $N\left(s_{i}, 0\right)=1$;

- Non annulation : en plus si $N(w, \chi)$ est défini, il est non nul. 


\subsection{Premier exemple de calculs, cas où $\pi$ est symétrique}

Soit $\left(\alpha^{+}, \alpha^{-}, \beta\right) \in \mathcal{A}^{\text {st }}(n)$ tel que $\alpha^{-}=\varnothing, \alpha^{+}$réduit à un seul terme, disons $\alpha$, de sorte que l'on a :

$$
\pi=\mathrm{St}_{\beta_{1}} \times \cdots \times \mathrm{St}_{\beta_{t}} \times \mathrm{St}_{\alpha} \times \mathrm{St}_{\beta_{t}} \times \cdots \times \mathrm{St}_{\beta_{1}}
$$

Notons que le caractère $\eta$ défini en 2.1.3 est alors $\theta$-invariant. On dit que $\pi$ est symétrique.

2.4.1. Détermination de l'opérateur $A: \pi \simeq \pi^{\theta}$. - Comme on a dit, la représentation $(\pi, V)$ peut être réalisée comme une sous-représentation de $\operatorname{Ind}_{B}^{G}(\eta)$. Notons $I(\eta)$ l'espace de représentation de $\operatorname{Ind}_{B}^{G}(\eta)$, qui est un sous-espace de $C^{\infty}(G)$ l'ensemble des fonctions localement constantes sur $G$. Pour $f \in C^{\infty}(G)$, on a posé : $f^{\theta}(g)=f(\theta(g))$. Soit $A^{\theta}$ l'application définie par

$$
\begin{aligned}
A^{\theta}: C^{\infty}(G) & \longrightarrow C^{\infty}(G) \\
f & \longmapsto f^{\theta}
\end{aligned}
$$

Pour $(\pi, V)$ une représentation de $G$, notons $\left(\pi^{\theta}, V\right)$ la représentation définie par $\pi^{\theta}(g)=\pi(\theta(g))$. Le fait suivant est facile à démontrer :

LEMME 2.9. - L'application $A^{\theta}$ réalise un opérateur d'entrelacement entre les représentations $\operatorname{Ind}_{B}^{G}(\eta)$ et $\operatorname{Ind}_{B}^{G}\left(\eta^{\theta}\right)$.

Comme $\eta$ est $\theta$-invariant, la restriction de $A^{\theta}$ à $V$ entrelace $\pi$ et $\pi^{\theta}$, et est donc un multiple de $A$. Montrons que :

$$
A=A_{\mid V}^{\theta}
$$

Comme ce sont des opérateurs proportionnels, et puisque $A$ fixe la droite $D$, il suffit de montrer que $A_{\mid V}^{\theta}$ fixe cette droite aussi. Par définition, la droite $D$ est engendrée par :

$$
\delta=\sum_{w \in W}(-q)^{-\ell(w)} T_{w}
$$

On vérifie facilement par les définitions que pour tout $w \in W, A^{\theta}\left(T_{w}\right)=\theta\left(T_{w}\right)=$ $T_{\theta(w)}$. Mais on a $\ell(w)=\ell(\theta(w))$, d'où le résultat.

2.4.2. Action de $A$ sur les modules de Jacquet $\pi_{N}, \mathbf{P}=\mathbf{M N} \in \mathcal{P}^{\theta}$. - Rappelons que pour calculer des traces tordues compactes, on dispose de la formule 1.7. Il nous faut donc des informations sur l'action de $A$ sur les modules de Jacquet de $\pi$. Soit $\mathbf{P}=\mathbf{M N}$ un sous-groupe parabolique standard $\theta$-stable. On a montré que le module de Jacquet $\pi_{N}$ admet une filtration, dite de Bernstein-Zelevinsky, indexée par l'ensemble $\left[W^{M} \backslash W / W^{L}\right]$. Soit $w \in W$, notons $\theta(w)$ le conjugué de $w$ par l'élément de plus grande longueur de $W$.

Lemme 2.10. - L'action de A respecte la filtration de Bernstein-Zelevinsky. Son action sur le gradué $J H\left(\pi_{N}\right)$ est telle que : 
(1) $A: F_{w}(\pi) \longrightarrow F_{\theta(w)}(\pi)$;

(2) Supposons que $w=\theta(w)$, alors $A$ agit par la formule 2.8 sur la composante $F_{w}(\pi)$.

En effet, cela vient de la description de $A$ donnée ci-dessus et celle de la filtration de Bernstein-Zelevinsky rappelée en 2.2 .

2.4.3. Calcul de $\operatorname{tr}_{\theta-c} \pi$. - Soit $f \in \mathcal{H}$. En vertu de la formule 1.7, le calcul de $\operatorname{tr}_{\theta-c} \pi(f)$ se ramène à celui de $\operatorname{tr}_{\theta} \pi_{N}\left(\widehat{\chi}_{N}^{\theta} f^{\theta, P}\right)$ pour $\mathbf{P}$ parcourant l'ensemble des sous-groupes paraboliques standards $\theta$-stables de $\mathbf{G}$. Soit donc $\mathbf{P} \in \mathcal{P}^{\theta}$. Remarquons que pour $f \in \mathcal{H}$, la fonction $f^{\theta, P}$ introduite en 1.3 devient :

$$
f^{\theta, P}(m)=\delta_{P}^{1 / 2}(m) \int_{N} f(m n) d n
$$

Ce n'est autre que le terme constant de $f$ le long de $P$. On la note simplement par $f^{P}$. La fonction $\hat{\chi}_{N}^{\theta} f^{\theta, P}=\widehat{\chi}_{N}^{\theta} f^{P}$ dans la formule 1.7 est un élément de $\mathcal{H}(M)$. Par conséquent, seuls les vecteurs sphériques non nuls de $V_{N}$ (les vecteurs non nuls qui sont invariants sous $\mathcal{H}(M))$ sont en jeu. On a posé :

$$
\left[W^{M} \backslash W / W^{L}\right]^{0}=\left\{w \in\left[W^{M} \backslash W / W^{L}\right] ; W^{M} \cap w W_{0} w^{-1}=1\right\}
$$

On a le résultat suivant :

Lemme 2.11. - Soit $w \in\left[W^{M} \backslash W / W^{L}\right]$, alors le gradué $F_{w}(\pi)$ a des vecteurs sphériques non nuls si et seulement si $w \in\left[W^{M} \backslash W / W^{L}\right]^{0}$.

Démonstration. - En effet, par définition, la représentation $\pi$ est l'induite d'une représentation $\rho$ de $L$, qui est le produit tensoriel des Steinberg ou leurs torsions par $\xi$.

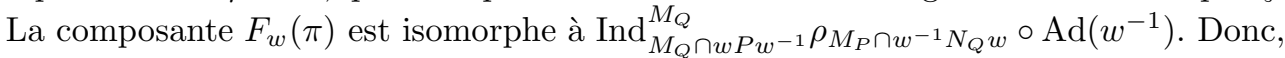
le module de Jacquet $\rho_{M_{P} \cap w^{-1} N_{Q} w}$ est aussi un produit tensoriel des torsions des Steinberg par des caractères non ramifiés. Par suite, $F_{w}(\pi)$ a des vecteurs sphériques non nuls si et seulement si $L \cap w^{-1} N w=B$, mais cette condition est équivalente à celle du lemme.

Le résultat ci-dessus, combiné avec le lemme 2.10, montre que seules contribuent à la trace les composantes $F_{w}(\pi)$ telles que $w=\theta(w)$ et $w \in\left[W^{M} \backslash W / W^{L}\right]^{0}$. Notons $\left[W^{M} \backslash W / W^{L}\right]^{0, \theta}$ cet ensemble.

Proposition 2.12. - Soit $\varphi$ un élément de $\mathcal{H}(M)$, et soit $S(\varphi)$ sa transformée de Satake. On a l'égalité :

$$
\operatorname{tr}_{\theta}\left(\pi_{N}\right)(\varphi)=\sum_{w \in\left[W^{M} \backslash W / W^{L}\right]^{0, \theta}} S(\varphi)\left(q^{-\eta_{w(1)}}, \ldots, q^{-\eta_{w(2 n+1)}}\right)
$$

Démonstration. - Il suffit d'utiliser ce qui précède et une formule bien connue de Van Dijk (voir [Van, Th.2]). 
REMARQue 2.14. - On peut obtenir les lemmes 2.10, 2.11 et la proposition 2.12 en utilisant la description de l'espace $V^{I}$ comme modules sur l'algèbre de Hecke-Iwahori (voir $[\mathbf{W a 1}]$ ).

La formule 2.13, appliquée à $\varphi=\widehat{\chi}_{N}^{\theta} f^{P}$, jointe avec la formule 1.7 calculent la trace tordue compacte $\operatorname{tr}_{\theta-c} \pi$ sur l'algèbre de Hecke sphérique. 


\title{
CHAPITRE 3
}

\author{
LE CAS $G L(5)$
}

Dans ce chapitre, G désigne le groupe $\mathbf{G L}(5)$.

\subsection{Calculs explicites dans les cas symétriques}

Il s'agit de quatre cas suivants :

- Cas $1:\left(\alpha^{+}, \alpha^{-}, \beta\right)=((5), \varnothing, \varnothing)$, ce qui correspond à $\pi=\mathrm{St}_{5}$;

- Cas $2:\left(\alpha^{+}, \alpha^{-}, \beta\right)=((3), \varnothing,(1))$, ce qui correspond à $\pi=\mathrm{St}_{1} \times \mathrm{St}_{3} \times \mathrm{St}_{1}$;

- Cas $3:\left(\alpha^{+}, \alpha^{-}, \beta\right)=((1), \varnothing,(2))$, ce qui correspond à $\pi=\mathrm{St}_{2} \times \mathrm{St}_{1} \times \mathrm{St}_{2}$;

- Cas $4:\left(\alpha^{+}, \alpha^{-}, \beta\right)=((1), \varnothing,(1,1))$, ce qui correspond à

$$
\pi=\mathrm{St}_{1} \times \mathrm{St}_{1} \times \mathrm{St}_{1} \times \mathrm{St}_{1} \times \mathrm{St}_{1}
$$

3.1.1. Transformée de Satake de $\hat{\chi}_{N}^{\theta} f^{\theta, P}$. Soit $\mathbf{P}=\mathbf{M N}$ un sous-groupe parabolique standard de $\mathbf{G}$, on dispose alors d'un digramme commutatif suivant :

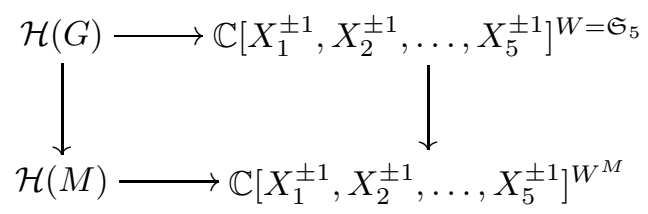

où les flèches horizontales sont des isomorphismes de Satake relatifs à $G$ et $M$, la première flèche verticale est donnée par le terme constant et finalement la dernière flèche est l'injection triviale.

Le lemme suivant est élémentaire :

Lemme 3.1. - Soit $f \in \mathcal{H}(G)$, et écrivons :

$$
S(f)\left(X_{1}, \ldots, X_{5}\right)=\sum_{\boldsymbol{m}=\left(m_{1}, \ldots, m_{5}\right) \in \mathbb{Z}^{5}} c_{\boldsymbol{m}} X_{1}^{m_{1}} \cdots X_{5}^{m_{5}}
$$

Alors la transformée de Satake de la fonction $\widehat{\chi}_{P}^{\theta} f^{\theta, P}$ dans la formule 1.7 est égale à 
(1) $\sum_{\substack{\boldsymbol{m} \in \mathbb{Z}^{5} \\ m_{1}-m_{5}>0}} c_{\boldsymbol{m}} X_{1}^{m_{1}} \cdots X_{5}^{m_{5}}$, si $\mathbf{P}=\mathbf{P}_{(1,3,1)}$ le parabolique standard paramétré par la partition $(1,3,1)$;

(2) $\sum_{\boldsymbol{m} \in \mathbb{Z}^{5}} c_{\boldsymbol{m}} X_{1}^{m_{1}} \cdots X_{5}^{m_{5}}$, si $\mathbf{P}=\mathbf{P}_{(2,1,2)}$;

(3) $\sum \underset{\substack{\boldsymbol{m} \in \mathbb{Z}^{5} \\ m_{1}-m_{5}>0}}{\operatorname{mon}} X_{1}^{m_{1}} \cdots X_{5}^{m_{5}}$, si $\mathbf{P}=\mathbf{B}$.

$m_{1}+m_{2}-m_{4}-m_{5}>0$

3.1.2. Calculs explicites de $\operatorname{tr}_{\theta-c} \pi$. - Le résultat de nos calculs se résume dans la proposition suivante :

Proposition 3.2. - Soit $f \in \mathcal{H}(G)$ dont l'image par isomorphisme de Satake est donnée par la formule :

$$
S(f)\left(X_{1}, \ldots, X_{5}\right)=\sum_{\boldsymbol{m}=\left(m_{1}, \ldots, m_{5}\right) \in \mathbb{Z}^{5}} c_{\boldsymbol{m}} X_{1}^{m_{1}} \cdots X_{5}^{m_{5}}
$$

Alors, la trace $\operatorname{tr}_{\theta-c} \pi(f)$ est égale à

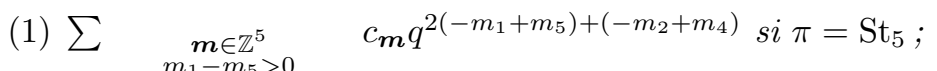

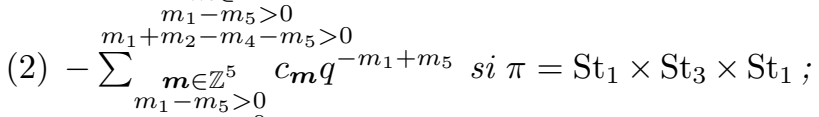

(3) $-\sum_{\boldsymbol{m} \in \mathbb{Z}^{5}}^{m_{2}-m_{4}=0} \quad c_{\boldsymbol{m}} q^{-m_{1}+m_{5}}$ si $\pi=\mathrm{St}_{2} \times \mathrm{St}_{1} \times \mathrm{St}_{2}$;

(4) $\sum_{m_{1}-m_{5}=m_{2}-m_{4}=0}^{\substack{\boldsymbol{m} \in \mathbb{Z}^{5} \\ m_{\boldsymbol{m}}}}$ si $\pi=\mathrm{St}_{1} \times \mathrm{St}_{1} \times \mathrm{St}_{1} \times \mathrm{St}_{1} \times \mathrm{St}_{1}$.

Démonstration. - Les sous-groupes paraboliques standards $\theta$-stables sont :

$$
\mathcal{P}^{\theta}=\left\{\mathbf{G}, \mathbf{P}_{1,3,1}, \mathbf{P}_{2,1,2}, \mathbf{B}\right\}
$$

La formule 1.7 s'écrit donc :

$$
\begin{aligned}
\operatorname{tr}_{\theta-c} \pi(f)= & \operatorname{tr}_{\theta} \pi(f)-\operatorname{tr}_{\theta} \pi_{N_{(1,3,1)}}\left(\widehat{\chi}_{N_{(1,3,1)}} f^{P_{(1,3,1)}}\right) \\
& -\operatorname{tr}_{\theta} \pi_{N_{(2,1,2)}}\left(\widehat{\chi}_{N_{(2,1,2)}} f^{P_{(2,1,2)}}\right)+\operatorname{tr}_{\theta} \pi_{U}\left(\widehat{\chi}_{U}^{\theta} f^{U}\right)
\end{aligned}
$$

On va appliquer la proposition 2.12 et le lemme 3.1 pour calculer les termes du membre de droite de la formule ci-dessus.

3.1.2.1. Démonstration dans le cas $\pi=\mathrm{St}_{5}$. - On montre facilement que $\left[W^{M} \backslash W / W^{L}\right]^{0}=\varnothing$ si $\mathbf{P}=\mathbf{G}, \mathbf{P}_{\mathbf{1}, \mathbf{3}, \mathbf{1}}, \mathbf{P}_{\mathbf{2}, \mathbf{1}, \mathbf{2}}$. D'où :

$$
\operatorname{tr}_{\theta} \pi(f)=\operatorname{tr}_{\theta} \pi_{N_{(1,3,1)}}\left(\widehat{\chi}_{N_{(1,3,1)}}^{\theta} f^{P_{(1,3,1)}}\right)=\operatorname{tr}_{\theta} \pi_{N_{(2,1,2)}}\left(\widehat{\chi}_{N_{(2,1,2)}}^{\theta} f^{P_{(2,1,2)}}\right)=0
$$

Enfin, pour $\mathbf{P}=\mathbf{B}$, on a $\left[W^{M} \backslash W / W^{L}\right]^{0}=\left[W^{M} \backslash W / W^{L}\right]^{0, \theta}=\{1\}, \pi_{U}=\eta=$ $(2,1,0,-1,-2)$. La formule 3.3 s'écrit :

$$
\operatorname{tr}_{\theta-c} \operatorname{St}_{5}(f)=\operatorname{tr}_{\theta} \eta\left(\widehat{\chi}_{U}^{\theta} f^{B}\right)
$$

Il suffit alors d'appliquer le lemme 3.1 pour obtenir le résultat voulu. 
3.1.2.2. Démonstration dans le cas $\pi=\mathrm{St}_{1} \times \mathrm{St}_{3} \times \mathrm{St}_{1}$

Pour $\mathbf{P}=\mathbf{G},\left[W^{M} \backslash W / W^{L}\right]^{0}=\varnothing$, d'où :

$$
\operatorname{tr}_{\theta} \pi(f)=0
$$

Pour $\mathbf{P}=\mathbf{P}_{2,1,2}$, on a $\left[W^{M} \backslash W / W^{L}\right]^{0, \theta}=\left\{w_{1}, w_{2}\right\}$ où :

$$
w_{1}=1, \quad w_{2}=\left(\begin{array}{lllll}
1 & 2 & 3 & 4 & 5 \\
4 & 1 & 3 & 5 & 2
\end{array}\right)
$$

Par application de la formule 2.13 , on obtient :

$$
\operatorname{tr}_{\theta} \pi_{N_{(2,1,2)}}\left(\widehat{\chi}_{N_{(2,1,2)}} f^{P_{(2,1,2)}}\right)=2 \sum_{\substack{\boldsymbol{m} \in \mathbb{Z}^{5} \\ m_{1}+m_{2}-m_{4}-m_{5}>0}} c_{\boldsymbol{m}} q^{-m_{1}+m_{5}}
$$

Pour $\mathbf{P}=\mathbf{P}_{(\mathbf{1 , 3}, \mathbf{1})}$, on a $\left[W^{M} \backslash W / W^{L}\right]^{0, \theta}=\left\{w_{3}\right\}$ où :

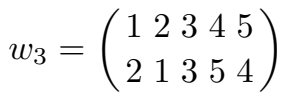

En appliquant la formule 2.13, on trouve :

$$
\operatorname{tr}_{\theta} \pi_{N_{(1,3,1)}}\left(\widehat{\chi}_{N_{(1,3,1)}}^{\theta} f^{\left.P_{(1,3,1)}\right)}=\sum_{\substack{\boldsymbol{m} \in \mathbb{Z}^{5} \\ m_{1}-m_{5}>0}} c_{\boldsymbol{m}} q^{-m_{1}+m_{5}}\right.
$$

Pour $\mathbf{P}=\mathbf{B}$, on a : $\left[W^{M} \backslash W / W^{L}\right]^{0, \theta}=\left\{w_{4}, w_{5}, w_{6}, w_{7}\right\}$, où :

$$
w_{4}=1, \quad w_{5}=\left(\begin{array}{lllll}
1 & 2 & 3 & 4 & 5 \\
5 & 2 & 3 & 4 & 1
\end{array}\right), \quad w_{6}=\left(\begin{array}{lllll}
1 & 2 & 3 & 4 & 5 \\
2 & 1 & 3 & 5 & 4
\end{array}\right), \quad w_{7}=\left(\begin{array}{lllll}
1 & 2 & 3 & 4 & 5 \\
4 & 1 & 3 & 5 & 2
\end{array}\right)
$$

Appliquons encore une fois la formule 2.13. On a :

$$
\operatorname{tr}_{\theta} \pi_{U}\left(\widehat{\chi}_{U}^{\theta} f^{B}\right)=2 \sum_{\substack{\boldsymbol{m} \in \mathbb{Z}^{5} \\ m_{1}-m_{5}>0 \\ m_{1}+m_{2}-m_{4}-m_{5}>0}} c_{\boldsymbol{m}} q^{-m_{1}+m_{5}}+2 \sum_{\substack{\boldsymbol{m} \in \mathbb{Z}^{5} \\ m_{1}-m_{5}>0 \\ m_{1}+m_{2}-m_{4}-m_{5}>0}} c_{\boldsymbol{m}} q^{-m_{2}+m_{4}}
$$

D'après les identités 3.3 à 3.7 , on a :

$$
\begin{aligned}
\operatorname{tr}_{\theta-c} \pi(f)= & -2 \sum_{\substack{\boldsymbol{m} \in \mathbb{Z}^{5} \\
m_{1}+m_{2}-m_{4}-m_{5}>0}} c_{\boldsymbol{m}} q^{-m_{1}+m_{5}}-\sum_{\substack{\boldsymbol{m} \in \mathbb{Z}^{5} \\
m_{1}-m_{5}>0}} c_{\boldsymbol{m}} q^{-m_{1}+m_{5}}+ \\
& +2 \sum_{\substack{\boldsymbol{m} \in \mathbb{Z}^{5} \\
m_{1}-m_{5}>0 \\
m_{1}+m_{2}-m_{4}-m_{5}>0}} c_{\boldsymbol{m}} q^{-m_{1}+m_{5}}+2 \sum_{\substack{\boldsymbol{m} \in \mathbb{Z}^{5} \\
m_{1}-m_{5}>0 \\
m_{1}+m_{2}-m_{4}-m_{5}>0}} c_{\boldsymbol{m}} q^{-m_{2}+m_{4}}
\end{aligned}
$$

Le reste est un problème combinatoire qui consiste à récrire la formule ci-dessus sous la forme énoncée dans le théorème. Puisque $S(f)$ est invariante sous l'action du 
groupe $\mathfrak{S}_{5}$, on a $c_{m_{1}, m_{2}, m_{3}, m_{4}, m_{5}}=c_{m_{2}, m_{1}, m_{3}, m_{5}, m_{2}}$. Par conséquent, le dernier terme de la formule ci-dessus s'écrit :

$$
2 \sum_{\substack{\boldsymbol{m} \in \mathbb{Z}^{5} \\
m_{1}-m_{5}>0 \\
m_{1}+m_{2}-m_{4}-m_{5}>0}} c_{\boldsymbol{m}} q^{-\left(m_{2}-m_{4}\right)}=2 \sum_{\begin{array}{c}
m_{2}-m_{4}>0 \\
m_{1}+m_{2}-m_{4}-m_{5}>0
\end{array}} c_{\boldsymbol{m}} q^{-m_{1}+m_{5}}
$$

D'autre part, on a les décompositions :

$$
\begin{aligned}
& \left\{\boldsymbol{m} \in \mathbb{Z}^{5} ; m_{1}+m_{2}-m_{4}-m_{5}>0\right\} \\
& =\left\{\boldsymbol{m} \in \mathbb{Z}^{5} ; m_{1}-m_{5}>0, m_{1}+m_{2}-m_{4}-m_{5}>0\right\} \\
& \cup\left\{\boldsymbol{m} \in \mathbb{Z}^{5} ; m_{2}-m_{4}>0, m_{1}+m_{2}-m_{4}-m_{5}>0\right\}
\end{aligned}
$$

et

$$
\begin{aligned}
& \left\{\boldsymbol{m} \in \mathbb{Z}^{5} ; m_{1}-m_{5}>0, m_{1}+m_{2}-m_{4}-m_{5}>0\right\} \\
& \cap\left\{\boldsymbol{m} \in \mathbb{Z}^{5} ; m_{2}-m_{4}>0, m_{1}+m_{2}-m_{4}-m_{5}>0\right\} \\
& \quad=\left\{\boldsymbol{m} \in \mathbb{Z}^{5} ; m_{1}-m_{5}>0, m_{2}-m_{4}>0\right\}
\end{aligned}
$$

On en déduit :

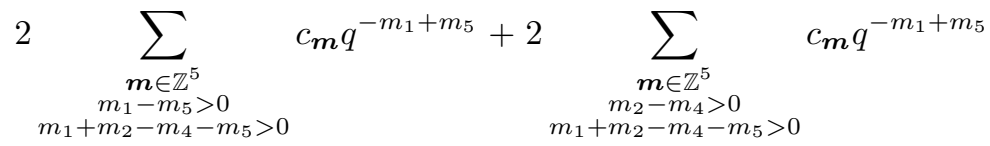

$$
\begin{aligned}
& =2 \sum_{\substack{\boldsymbol{m} \in \mathbb{Z}^{5} \\
m_{1}+m_{2}-m_{4}-m_{5}>0}} c_{\boldsymbol{m}} q^{-m_{1}+m_{5}}+2 \sum_{\substack{\boldsymbol{m} \in \mathbb{Z}^{5} \\
m_{1}-m_{5}>0}} c_{\boldsymbol{m}} q^{-m_{1}+m_{5}}
\end{aligned}
$$

Par suite,

$$
\operatorname{tr}_{\theta-c} \pi(f)=-\sum_{\substack{\boldsymbol{m} \in \mathbb{Z}^{5} \\ m_{1}-m_{5}>0}} c_{\boldsymbol{m}} q^{-m_{1}+m_{5}}+2 \sum_{\substack{\boldsymbol{m} \in \mathbb{Z}^{5} \\ m_{1}-m_{5}>0 \\ m_{2}-m_{4}>0}} c_{\boldsymbol{m}} q^{-m_{1}+m_{5}}
$$

Utilisons finalement la décomposition :

$$
\begin{aligned}
\left\{\boldsymbol{m} \in \mathbb{Z}^{5} ; m_{1}-m_{5}>0\right\}=\left\{\boldsymbol{m} \in \mathbb{Z}^{5} ; m_{1}-m_{5}>0, m_{2}-m_{4}>0\right\} \\
\sqcup\left\{\boldsymbol{m} \in \mathbb{Z}^{5} ; m_{1}-m_{5}>0, m_{2}-m_{4}<0\right\} \\
\sqcup\left\{\boldsymbol{m} \in \mathbb{Z}^{5} ; m_{1}-m_{5}>0, m_{2}-m_{4}=0\right\}
\end{aligned}
$$

et remarquons que :

$$
\sum_{\substack{\boldsymbol{m} \in \mathbb{Z}^{5} \\ m_{1}-m_{5}>0 \\ m_{2}-m_{4}>0}} c_{\boldsymbol{m}} q^{-m_{1}+m_{5}}=\sum_{\substack{\boldsymbol{m} \in \mathbb{Z}^{5} \\ m_{1}-m_{5}>0 \\ m_{2}-m_{4}<0}} c_{\boldsymbol{m}} q^{-m_{1}+m_{5}}
$$


cela par l'invariance de $S(f)$. Nous avons bien :

$$
\operatorname{tr}_{\theta-c} \pi(f)=-\sum_{\substack{\boldsymbol{m} \in \mathbb{Z}^{5} \\ m_{1}-m_{5}>0 \\ m_{2}-m_{4}=0}} c_{\boldsymbol{m}} q^{-m_{1}+m_{5}}
$$

Les autres cas se démontrent de façon similaire.

3.2. Cas $5: \pi=\mathrm{St}_{1} \times \xi \mathrm{St}_{3} \times \xi \mathrm{St}_{1}$

Il s'agit du cas où $\left(\alpha^{+}, \alpha^{-}, \beta\right)=((1),(3,1), \varnothing)$.

3.2.1. Construction de $A: \pi \simeq \pi^{\theta}$. — Dans ce paragraphe on construit $A$ comme dérivée des opérateurs d'entrelacement introduits en 2.3.9. Rappelons que l'on a posé : $\varepsilon=i \pi / \log q$. Soit $z$ une variable complexe, notons $\eta_{z}$ le caractère de $T$ défini par :

$$
\eta_{z}=(0,1+\varepsilon, \varepsilon,-1+\varepsilon, z+\varepsilon)
$$

Notons que pour $z=0$, on retrouve le caractère $\eta$ du tore diagonal défini en 2.1.3. On sait que $\pi \hookrightarrow \operatorname{Ind}_{B}^{G}(\eta)$. Plus précisément, posons $w_{1}=s_{2} s_{3} s_{2}$, alors d'après la théorie des opérateurs d'entrelacement $N\left(w_{1}, w_{1}^{-1} \eta\right)$ est bien défini dont l'image est la Steinberg généralisée $\pi$. Posons $w_{2}=(1,5)$ la transposition entre 1 et 5 . Pour $z \neq 0$ et assez proche de 0 , l'opérateur $N\left(w_{2}, \eta_{z}\right)$ est bien défini. Rappelons que $V$ désigne l'espace de représentation de $\pi$.

Lemme 3.8. - Pour tout $f \in V$, la limite $\lim _{z \rightarrow 0} N\left(w_{2}, \eta_{z}\right) f$ existe.

Démonstration. - Par la formule du produit et en écrivant $w_{2}=s_{4} s_{3} s_{2} s_{1} s_{2} s_{3} s_{4}$ on a :

$$
\begin{aligned}
N\left(w_{2}, \eta_{z}\right)=N\left(s_{4}, 1-\varepsilon\right) N\left(s_{3},-\varepsilon\right) N\left(s_{2},-\right. & 1-\varepsilon) N\left(s_{1},-\varepsilon-z\right) \\
& \times N\left(s_{2}, 1-z\right) N\left(s_{3},-z\right) N\left(s_{4},-1-z\right)
\end{aligned}
$$

Puisque $N\left(s_{i}, x\right)$ n'a qu'un pôle en $x=1$, il est clair que :

$$
\begin{aligned}
\lim _{z \rightarrow 0} N\left(s_{4}, 1-\varepsilon\right) N\left(s_{3},-\varepsilon\right) N\left(s_{2},-1-\varepsilon\right) N\left(s_{1},-\varepsilon-z\right) & =N\left(s_{4}, 1-\varepsilon\right) N\left(s_{3},-\varepsilon\right) \\
& \times N\left(s_{2},-1-\varepsilon\right) N\left(s_{1},-\varepsilon\right)
\end{aligned}
$$

Il reste à étudier le terme $N(z)=N\left(s_{2}, 1-z\right) N\left(s_{3},-z\right) N\left(s_{4},-1-z\right)$. Développons suivant $z$ au voisinage de zéro :

$$
\begin{aligned}
N\left(s_{2}, 1-z\right) & =-\frac{1}{z} N_{-1}\left(s_{2}, 1\right)+N_{0}\left(s_{2}, 1\right)+O(z) \\
N\left(s_{3},-z\right) & =N_{0}\left(s_{3}, 0\right)-z N_{1}\left(s_{3}, 1\right)+O\left(z^{2}\right) \\
N\left(s_{4},-1-z\right) & =N_{0}\left(s_{4},-1\right)-z N_{1}\left(s_{4},-1\right)+O\left(z^{2}\right)
\end{aligned}
$$


En remarquant que $N\left(s_{3}, 0\right)=1$, on peut donc écrire :

$$
\begin{aligned}
N(z)=-\frac{1}{z} N_{-1}\left(s_{2}, 1\right) N_{0}( & \left.s_{4},-1\right)+N_{-1}\left(s_{2}, 1\right) N_{1}\left(s_{3}, 0\right) N_{0}\left(s_{4},-1\right) \\
& +N_{-1}\left(s_{2}, 1\right) N_{1}\left(s_{4},-1\right)+N_{0}\left(s_{2}, 1\right) N_{1}\left(s_{4},-1\right)+O(z)
\end{aligned}
$$

Montrons que le terme $-\frac{1}{z} N_{-1}\left(s_{2}, 1\right) N_{0}\left(s_{4},-1\right)$ ne contribue pas dans la formule de $N\left(w_{2}, \eta_{z}\right) f$ si $f \in V$. Notons que si $|i-j|>2$, les opérateurs non normalisés $A\left(s_{i}\right)$ et $A\left(s_{j}\right)$ commutent, par conséquent, $N\left(s_{i}, s_{j} \eta\right) N\left(s_{j}, \eta\right)=N\left(s_{j}, s_{i} \eta\right) N\left(s_{i}, \eta\right)$. On a donc $N_{-1}\left(s_{2}, 1\right) N_{0}\left(s_{4},-1\right)=N_{0}\left(s_{4},-1\right) N_{-1}\left(s_{2}, 1\right)$. Il suffit donc de vérifier que pour tout $f \in V$, on a :

$$
N_{0}\left(s_{4},-1\right) N_{-1}\left(s_{2}, 1\right) f=0
$$

On a déjà vu que les représentations de Steinberg peuvent être définies comme images de certains opérateurs d'entrelacement, on peut écrire

$$
f=N\left(s_{2},-1\right) N\left(s_{3},-2\right) N\left(s_{2},-1\right) \phi
$$

pour un $\phi \in J$. On va en fait montrer que :

$$
N_{-1}\left(s_{2}, 1\right) N_{0}\left(s_{2},-1\right)=0
$$

Pour cela, il suffit de remarquer que pour tout $z$, par la formule du produit, on a l'égalité :

$$
N\left(s_{2}, 1-z\right) N\left(s_{2},-1+z\right)=1
$$

Par suite, en développant suivant $z$, on a :

$$
-\frac{1}{z} N_{-1}\left(s_{2}, 1\right) N_{0}\left(s_{2},-1\right)+O(1)=1
$$

Cela étant pour tout $z$, d'où $N_{-1}\left(s_{2}, 1\right) N_{0}\left(s_{2},-1\right)=0$, comme attendu.

De même, le terme $N_{-1}\left(s_{2}, 1\right) N_{0}\left(s_{4},-1\right)$ dans le développement de $N(z)$ ne contribue pas dans la formule de $N\left(w_{2}, \eta_{z}\right) f$ si $f \in V$.

On a ainsi montré que pour $f \in V, \lim _{z \rightarrow 0} N\left(w_{2}, \eta_{z}\right) f$ existe et vaut

$$
\begin{aligned}
N\left(w_{2}\right) f=N\left(s_{4}, 1-\varepsilon\right) N\left(s_{3},-\varepsilon\right) N\left(s_{2},-1-\varepsilon\right) N\left(s_{1},-\varepsilon\right) & \\
& \times\left(N_{-1}\left(s_{2}, 1\right) N_{1}\left(s_{3}, 0\right) N_{0}\left(s_{4},-1\right)+N_{0}\left(s_{2}, 1\right) N_{0}\left(s_{4},-1\right)\right) f
\end{aligned}
$$

Soit $\left(\pi^{\prime}, W\right)$ la représentation $\xi \mathrm{St}_{1} \times \xi \mathrm{St}_{3} \times \mathrm{St}_{1}$, on a alors le lemme suivant :

Lemme 3.9

(1) Pour tout $f \in V$, alors $N\left(w_{2}\right) f \in W$.

(2) L'opérateur $N\left(w_{2}\right)$ est un isomorphisme entre $V$ et $W$.

(3) Par conséquent, $N\left(w_{2}\right)$ définit un opérateur d'entrelacement entre $\pi$ et $\pi^{\prime}$.

Démonstration. - On a déjà dit que $V$ est l'image de l'opérateur $N\left(w_{1}, w_{1}^{-1} \eta\right)$. D'autre part, on peut vérifier d'après les définitions que $N\left(w_{1}, w_{1}^{-1} \eta\right)=N\left(w_{1}, w_{1}^{-1} \eta_{z}\right)$. 
Écrivons alors $f=N\left(w_{1}, w_{1}^{-1} \eta\right) \phi=\lim _{z \rightarrow 0} N\left(w_{1}, w_{1}^{-1} \eta_{z}\right) \phi$ avec un $\phi \in J$. Par définition de $N\left(w_{2}\right)$, on a :

$$
\begin{aligned}
N\left(w_{2}\right) f & =\lim _{z \rightarrow 0} N\left(w_{2}, \eta_{z}\right) N\left(w_{1}, w_{1}^{-1} \eta_{z}\right) \phi \\
& =\lim _{z \rightarrow 0} N\left(w_{2} w_{1}, w_{1}^{-1} \eta_{z}\right) \phi \\
& =\lim _{z \rightarrow 0} N\left(w_{1} w_{2}, w_{1}^{-1} \eta_{z}\right) \phi \quad \operatorname{car} w_{1} w_{2}=w_{2} w_{1} \\
& =\lim _{z \rightarrow 0} N\left(w_{1}, w_{2} w_{1}^{-1} \eta_{z}\right) N\left(w_{2}, w_{1}^{-1} \eta_{z}\right) \phi
\end{aligned}
$$

On peut vérifier également par les définitions que $N\left(w_{1}, w_{2} w_{1}^{-1} \eta_{z}\right)$ est indépendant de $z$. Or l'image de cet opérateur est précisément $W$, d'où le premier point du lemme.

Pour le second point, par la même méthode que celle du lemme précédent, on montre que si $\psi \in W$, alors $\lim _{z \rightarrow 0} N\left(w_{2}^{-1}, w_{2} \eta_{z}\right) \psi$ existe et vaut, disons $N\left(w_{2}^{-1}\right) \psi$. Il suffit alors de développer suivant $z$ l'égalité $N\left(w_{2}^{-1}, w_{2} \eta_{z}\right) N\left(w_{2}, \eta_{z}\right)=1$, puis comparer les puissances de $z$ pour avoir l'égalité $N\left(w_{2}^{-1}\right) N\left(w_{2}\right)=1$. De façon similaire, on montre que $N\left(w_{2}\right) N\left(w_{2}^{-1}\right)=1$, d'où le résultat.

Enfin, montrons le dernier point. D'après les propriétés des opérateurs d'entrelacement, $N\left(w_{2}, \eta_{z}\right)$ entrelace $\operatorname{Ind}_{B}^{G}\left(\eta_{z}\right)$ et $\operatorname{Ind}_{B}^{G}\left(w_{2} \eta_{z}\right)$, si $z \neq 0$ est assez proche de 0 . Par prolongement en 0 de la restriction de $N\left(w_{2}, \eta_{z}\right)$ à $V$, l'opérateur $N\left(w_{2}, \eta\right)$ entrelace la représentation $\pi$ et son image, c'est-à-dire $\pi^{\prime}$. C'est un isomorphisme d'après ce qui précède, d'où le résultat.

\section{Proposition 3.10}

(1) L'opérateur $A^{\theta} \circ N\left(w_{2}\right)$ est défini sur $V$ et réalise un opérateur d'entrelacement entre $(\pi, V)$ et $\left(\pi^{\theta}, V\right)$.

(2) On $a: A=A^{\theta} \circ N\left(w_{2}\right)_{\mid V}$.

Démonstration. - Le premier point résulte de ci-dessus. Par suite, $A^{\theta} \circ N\left(w_{2}\right)$ est un multiple de $A$. D'autre part, d'après notre normalisation, les opérateurs d'entrelacement fixent la droite $D$ donc $A^{\theta} \circ N\left(w_{2}\right)$ fixe $D$, d'où le résultat.

\subsubsection{Action de $A$ sur le module de Jacquet $\pi_{U}$}

3.2.2.1. Décomposition de $V_{U}$ en somme de sous-espaces caractéristiques. - Pour tout caractère complexe $\chi$ de $T$, définissons l'espace caractéristique de $V_{U}$ relatif à $\chi$ par :

$$
V_{U}[\chi]=\left\{v \in V_{U} ; \exists \text { un entier } i \text { tel que }\left(\pi_{U}(t)-\chi(t)\right)^{i}=0 \text { pour tout } t \in T\right\}
$$

On a alors la décomposition de $V_{U}$ en somme de ses sous-espaces caractéristiques :

$$
V_{U}=\oplus_{\chi} V_{U}[\chi]
$$

On vérifie aisément que $A\left(V_{U}[\chi]\right) \subset V_{U}[\theta(\chi)]$. Par conséquent, seuls les caractères $\chi$ tels que $\chi=\theta(\chi)$ contribuent à la trace tordue. Dans notre cas, par application de la 
filtration de Bernstein-Zelevinsky, seul le caractère $\zeta=(1+\varepsilon, \varepsilon, 0, \varepsilon,-1+\varepsilon)$ intervient dans nos calculs, en plus $V_{U}[\zeta]$ est de dimension 2.

3.2.2.2. Action de $T$ sur $V_{U}[\zeta]$. - Posons :

$$
w^{\prime}=\left(\begin{array}{lllll}
1 & 2 & 3 & 4 & 5 \\
3 & 1 & 2 & 5 & 4
\end{array}\right) \quad \text { et } \quad w^{\prime \prime}=\left(\begin{array}{lllll}
1 & 2 & 3 & 4 & 5 \\
3 & 1 & 4 & 5 & 2
\end{array}\right)
$$

alors $\zeta=w^{\prime} \eta=w^{\prime \prime} \eta$. Plus généralement, soit $z$ une variable complexe, posons $\zeta_{z}^{\prime}=$ $w^{\prime} \eta_{z}, \zeta_{z}^{\prime \prime}=w^{\prime \prime} \eta_{z}$. Remarquons que si $z \neq 0, \eta_{z}$ est régulier. On vérifie (par la formule du produit, par exemple) que les opérateurs $N\left(w^{\prime}, \eta\right), N\left(w^{\prime \prime}, \eta\right)$ sont définis et opposés. Par suite, la limite $\lim _{z \rightarrow 0} \frac{1}{z}\left(N\left(w^{\prime}, \eta_{z}\right)+N\left(w^{\prime \prime}, \eta_{z}\right)\right)$ existe. Posons :

$$
\alpha(f)=N\left(w^{\prime}, \eta\right) f(1) \quad \text { et } \quad \beta(f)=\lim _{z \rightarrow 0} \frac{1}{z}\left(N\left(w^{\prime}, \eta_{z}\right)+N\left(w^{\prime \prime}, \eta_{z}\right)\right) f(1)
$$

On a : $\alpha \neq 0$ car $N\left(w^{\prime}, \eta\right) N\left(w_{1}, w_{1} \eta\right)$ est défini et non nul. Soit $t \in T$ et écrivons $t \cdot f$ au lieu de $\pi_{U}(t)(f)$, il est clair que :

$$
\alpha(t \cdot f)=\zeta(t) \alpha(f)
$$

Quant à $\beta(t \cdot f)$, on a :

$$
\begin{aligned}
\beta(t \cdot f)= & \lim _{z \rightarrow 0} \frac{1}{z}\left[N\left(w^{\prime}, \eta_{z}\right)(t \cdot f)(1)+N\left(w^{\prime \prime}, \eta_{z}\right)(t \cdot f)(1)\right] \\
= & \lim _{z \rightarrow 0} \frac{1}{z}\left[w^{\prime} \eta_{z}(t) N\left(w^{\prime}, \eta_{z}\right) f(1)+w^{\prime \prime} \eta_{z}(t) N\left(w^{\prime \prime}, \eta_{z}\right) f(1)\right] \\
= & \lim _{z \rightarrow 0} \frac{1}{z}\left[\left(w^{\prime} \eta_{z}(t)-w^{\prime \prime} \eta_{z}(t)\right) N\left(w^{\prime}, \eta_{z}\right) f(1)\right. \\
& \left.\quad+w^{\prime \prime} \eta_{z}(t)\left(N\left(w^{\prime}, \eta_{z}\right)+N\left(w^{\prime \prime}, \eta_{z}\right)\right) f(1)\right]
\end{aligned}
$$

Quand $z$ tend vers 0 , la $\operatorname{limite}_{\lim _{z \rightarrow 0}} \frac{1}{z}\left(w^{\prime} \eta_{z}(t)-w^{\prime \prime} \eta_{z}(t)\right)$ existe. Notons $c(t)$ sa valeur. Cette fonction est non nulle. D'autre part, $\lim _{z \rightarrow 0} w^{\prime \prime} \eta_{z}(t)=w^{\prime \prime} \eta(t)=\zeta(t)$. On obtient :

$$
\beta(t \cdot f)=c(t) \alpha(f)+\zeta(t) \beta(f)
$$

Puisque $c$ est non nulle, cette égalité montre que $\beta$ n'est pas proportionnel à $\alpha$. On en déduit :

Lemme 3.11. - L'application

$$
\begin{aligned}
(\alpha, \beta): V & \longrightarrow \mathbb{C}^{2} \\
f & \longmapsto(\alpha(f), \beta(f))
\end{aligned}
$$

induit un isomorphisme entre $V_{U}[\zeta]$ et $\mathbb{C}^{2}$.

Identifions donc $V_{U}[\zeta]$ à $\mathbb{C}^{2}$ via $(\alpha, \beta)$. D'après les calculs qui précèdent le lemme ci-dessus, l'action de $T$ sur cet espace est de la forme :

$$
t \cdot\left(\begin{array}{l}
u \\
v
\end{array}\right)=\left(\begin{array}{ll}
\zeta(t) & 0 \\
c(t) & \zeta(t)
\end{array}\right)\left(\begin{array}{l}
u \\
v
\end{array}\right)
$$


3.2.2.3. Action de $A$ sur $V_{U}[\zeta]$. - Soit $f \in V$, on a :

$$
\begin{aligned}
\alpha \circ A(f) & =N\left(w^{\prime}, \eta\right) A^{\theta} \lim _{z \rightarrow 0} N\left(w_{2}, \eta_{z}\right) f(1) \\
& =A^{\theta}\left(N\left(\theta\left(w^{\prime}\right), \theta(\eta)\right) \lim _{z \rightarrow 0} N\left(w_{2}, \eta_{z}\right) f(1)\right. \\
& =N\left(\theta\left(w^{\prime}\right), \theta(\eta)\right) \lim _{z \rightarrow 0} N\left(w_{2}, \eta_{z}\right) f(1) \quad \operatorname{car} \theta(1)=1 ! \\
& =\lim _{z \rightarrow 0} N\left(\theta\left(w^{\prime}\right), w_{2} \eta_{z}\right) N\left(w_{2}, \eta_{z}\right) f(1) \quad \operatorname{car} \theta(\eta)=w_{2} \eta \\
& =\lim _{z \rightarrow 0} N\left(\theta\left(w^{\prime}\right) w_{2}, \eta_{z}\right) f(1) \\
& =N\left(w^{\prime \prime}, \eta\right) f(1)=\alpha(f) \quad \operatorname{car} \theta\left(w^{\prime}\right) w_{2}=w^{\prime \prime}
\end{aligned}
$$

La première égalité utilise implicitement le résultat suivant :

LEMME 3.12. - Soient $\chi$ un caractère non ramifié de $T$, et $w$ un élément du groupe de Weyl. Supposons que $N(w, \chi)$ est bien défini. Alors on a l'identité :

$$
N(w, \chi) A^{\theta}=A^{\theta} N(\theta(w), \theta(\chi))
$$

En effet, soit $f \in J$ montrons que lorsque $A(w) f$ converge (rappelons que $A(w)$ est l'opérateur d'entrelacement non normalisé), on a l'égalité :

$$
A(w) A^{\theta} f=A^{\theta} A(\theta(w)) f
$$

Soit donc $g \in G$, alors, par définition,

$$
\begin{aligned}
A^{\theta} A(\theta(w)) f(g) & =\int_{U \cap \theta(w) \bar{U} \theta\left(w^{-1}\right)} f\left(\theta\left(w^{-1}\right) u \theta(g)\right) d u \\
& =\int_{U \cap w \bar{U} w^{-1}} f\left(\theta\left(w^{-1}\right) \theta(u) \theta(g)\right) d u^{\prime} \quad \text { en posant } u^{\prime}=\theta(u) \\
& =\int_{U \cap w \bar{U} w^{-1}} A^{\theta} f\left(w^{-1} u^{\prime} g\right) d u^{\prime} \\
& =A(w) A^{\theta} f(g)
\end{aligned}
$$

Montrons que $c_{w}(\chi)=c_{\theta(w)}(\theta(\chi))$. Par définition,

$$
c_{\theta(w)}(\theta(\chi))=\prod_{\substack{i<j \\ \theta(w)(i)>\theta(w)(j)}} \frac{-1+q^{\theta(\chi)_{i}-\theta(\chi)_{j}}}{1-q^{-1+\theta(\chi)_{i}-\theta(\chi)_{j}}}
$$


Soit $w_{l}$ l'élément de plus grande longueur du groupe de Weyl, alors pour tout $i$, $\theta(\chi)_{i}=-\chi_{w_{l}(i)}$ et $\theta(w)=w_{l} w w_{l}$. On en déduit :

$$
\begin{aligned}
c_{\theta(w)}(\theta(\chi))= & \prod_{\substack{i<j \\
w_{l} w w_{l}(i)>w_{l} w w_{l}(j)}} \frac{-1+q^{-\chi_{w_{l}(i)}+\chi_{w_{l}(j)}}}{1-q^{-1+\chi-w_{l}(i)+\chi_{w_{l}(j)}}} \\
= & \prod_{\substack{i^{\prime}<j^{\prime} \\
w\left(i^{\prime}\right)>w\left(j^{\prime}\right)}} \frac{-1+q^{\chi_{i^{\prime}}-\chi_{j^{\prime}}}}{1-q^{-1+\chi_{i^{\prime}}-\chi_{j^{\prime}}}} \text { en posant } i^{\prime}=w_{l}(j), j^{\prime}=w_{l}(i) \\
= & c_{w}(\chi)
\end{aligned}
$$

On en déduit : $N(w, \chi) A^{\theta} f=A^{\theta} N(\theta(w), \theta(\chi)) f$ lorsque $A(w) f$ converge. Enfin, par prolongement, cette égalité est valable partout où elle est définie.

De façon similaire, on a aussi : $\beta \circ A(f)=\beta(f)$. On a ainsi montré :

Lemme 3.13. — L'opérateur $A$ agit trivialement sur le facteur $V_{U}[\zeta]$.

3.2.3. Action de $A$ sur $\pi_{N}$, où $\mathbf{P}=\mathbf{P}_{2,1,2}$ - - Par application de la filtration de Bernstein-Zelevinsky, et en considérant l'action du centre de $M=M_{(2,1,2)}$ sur ce module, on peut écrire :

$$
\pi_{N}=\operatorname{Ind}_{B}^{M}\left(\lambda_{1}\right) \oplus \operatorname{Ind}_{B}^{M}\left(\lambda_{2}\right) \oplus \nu
$$

avec $\lambda_{1}=(\varepsilon, 1+\varepsilon, \varepsilon, \varepsilon-1,0), \lambda_{2}=(1+\varepsilon, 0, \varepsilon, \varepsilon, \varepsilon-1)$ et $\nu$ est une représentation n'ayant pas de vecteurs sphériques non nuls. Par suite, $\pi_{N}$ a deux droites sphériques disons $D_{1}, D_{2}$ contenues respectivement dans $\operatorname{Ind}_{B}^{M}\left(\lambda_{1}\right)$ et $\operatorname{Ind}_{B}^{M}\left(\lambda_{2}\right)$. Il suffit alors de considérer l'action du centre de $M$ sur $D_{1}$ et $D_{2}$ pour voir que

LEMME 3.14. - On a :

$$
A\left(D_{1}\right)=D_{2} \quad \text { et } \quad A\left(D_{2}\right)=D_{1}
$$

3.2.4. Action de $A$ sur $\pi_{N}$, où $\mathbf{P}=\mathbf{P}_{1,3,1}$ • - Toujours par application de la filtration de Zelevinsky et en regardant l'action du centre de $M=M_{1,3,1}$, on peut écrire :

$$
\pi_{N}=\operatorname{Ind}_{B}^{M}(\zeta) \oplus \nu^{\prime}
$$

où $\nu^{\prime}$ est une représentation n'ayant pas de vecteurs sphériques non nuls, et $\zeta=$ $(1+\varepsilon, \varepsilon, 0, \varepsilon,-1+\varepsilon)$. Ainsi, $\pi_{N}$ n'admet qu'une seule droite sphérique, disons $D$ qui est contenue dans $\operatorname{Ind}_{B}^{M}(\zeta)$. Comme $A^{2}=\mathrm{Id}, A$ agit par \pm 1 sur $D$. Or, remarquons que $\left(\operatorname{Ind}_{B}^{M}(\zeta)\right)_{U}=\pi_{U}[\zeta]$ et que la projection de $D$ sur cet espace est non nulle. D'après ce qui précède, $A$ agit trivialement sur $\pi_{U}[\zeta]$, d'où :

Lemme 3.15. - L'opérateur A agit trivialement sur le vecteur sphérique D.

3.2.5. $\mathbf{P}=\mathbf{G L}(5)$. — Il suffit de remarquer que

LEMME 3.16. — La représentation $\pi$ n'a pas de vecteur sphérique non nul. 
3.2.6. Formule explicite de $\operatorname{tr}_{\theta-c} \pi$. - Il s'agit de la formule suivante :

Proposition 3.17 (Cas $\left.5: \pi=\mathrm{St}_{1} \times \xi \mathrm{St}_{3} \times \xi \mathrm{St}_{1}\right)$. - Soit $f \in \mathcal{H}(G)$ dont la transformée de Satake est donnée par :

$$
S(f)\left(X_{1}, \ldots, X_{5}\right)=\sum_{\boldsymbol{m}=\left(m_{1}, \ldots, m_{5}\right) \in \mathbb{Z}^{5}} c_{\boldsymbol{m}} X_{1}^{m_{1}} \cdots X_{5}^{m_{5}}
$$

On a l'égalité :

$$
\operatorname{tr}_{\theta-c} \pi(f)=-\sum_{\substack{\boldsymbol{m} \in \mathbb{Z}^{5} \\ m_{1}-m_{5}>0 \\ m_{2}=m_{4}}} c_{\boldsymbol{m}}(-q)^{-m_{1}+m_{5}}+2 \sum_{\substack{\boldsymbol{m} \in \mathbb{Z}^{5} \\ m_{1}-m_{5}>>\\>m_{2}-m_{4} \geqslant 0}} c_{\boldsymbol{m}}(-q)^{-m_{1}+m_{5}}(-1)^{-m_{2}+m_{4}}
$$

Démonstration. - D'après la formule 3.3 , les lemmes 3.14 et 3.16 , on a l'égalité :

$$
\operatorname{tr}_{\theta-c} \pi(f)=-\operatorname{tr}_{\theta} \pi_{N_{(1,3,1)}}\left(\widehat{\chi}_{N_{(1,3,1)}}^{\theta} f^{P_{(1,3,1)}}\right)+\operatorname{tr}_{\theta} \pi_{U}\left(\widehat{\chi}_{U}^{\theta} f^{B}\right)
$$

D'après les lemmes 3.15 et 3.1 ,

$$
\begin{aligned}
\operatorname{tr}_{\theta}\left(\widehat{\chi}_{N_{(1,3,1)}}^{\theta} f^{P_{(1,3,1)}}\right) & =\operatorname{tr} \operatorname{Ind}_{B}^{M_{(1,3,1)}}(\zeta)\left(\widehat{\chi}_{N_{(1,3,1)}}^{\theta} f^{P_{(1,3,1)}}\right) \\
& =-\sum_{\substack{\boldsymbol{m} \in \mathbb{Z}^{5}>0 \\
m_{1}-m_{5}>0}} c_{\boldsymbol{m}}(-q)^{-m_{1}+m_{5}}(-1)^{-m_{2}+m_{4}}
\end{aligned}
$$

D'autre part, d'après les lemmes 3.13 et 3.1 ,

$$
\begin{aligned}
& \operatorname{tr}_{\theta} \pi_{U}\left(\widehat{\chi}_{U}^{\theta} f^{B}\right)=2 \operatorname{tr} \zeta\left(\widehat{\chi}_{U}^{\theta} f^{B}\right) \\
& =2 \sum_{\substack{\boldsymbol{m} \in \mathbb{Z}^{5} \\
m_{1}-m_{5}>0 \\
m_{1}-m_{5}+m_{2}-m_{4}>0}} c_{\boldsymbol{m}}(-q)^{-m_{1}+m_{5}}(-1)^{-m_{2}+m_{4}}
\end{aligned}
$$

Le reste est un calcul combinatoire élémentaire que l'on va omettre.

\subsection{Transformation de Satake des traces tordues compactes}

Définissons un homomorphisme d'algèbres :

$$
p: \mathbb{C}\left[X_{1}^{ \pm 1}, \ldots, X_{5}^{ \pm 1}\right]^{\mathfrak{S}_{5}} \longrightarrow \mathbb{C}\left[X_{1}^{ \pm 1}, X_{2}^{ \pm 1}\right]^{W\left(C_{2}\right)}
$$

par $p\left(X_{1}\right)=p\left(X_{5}\right)^{-1}=X_{1}, p\left(X_{2}\right)=p\left(X_{4}\right)^{-1}=X_{2}, p\left(X_{3}\right)=1$. Cette application provient de l'endoscopie entre $\mathbf{G L}(5)$ et $\mathbf{S p}(\mathbf{4})$ (cf. Introduction). Posons :

$$
\widehat{T}_{u}=\left\{\left(x_{1}, x_{2}\right) \in \mathbb{C}^{2} ;\left|x_{1}\right|=\left|x_{2}\right|=1\right\}
$$

On a le résultat suivant :

ThÉORÈme 3.18. - Pour tout $f$ élément de $\mathcal{H}(G)$, on a l'égalité :

$$
\operatorname{tr}_{\theta-c} \pi(f)=\int_{\widehat{T}_{u}} p(S(f))\left(x_{1}, x_{2}\right) Q_{\pi}\left(x_{1}, x_{2}\right) d^{\times} x_{1} d^{\times} x_{2}
$$

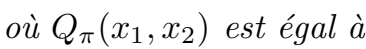




$$
\begin{array}{lll}
\text { - } & \text { si } \pi=\mathrm{St}_{1} \times \mathrm{St}_{1} \times \mathrm{St}_{1} \times \mathrm{St}_{1} \times \mathrm{St}_{1} ; \\
\text { - } & \text { si } \pi=\mathrm{St}_{2} \times \mathrm{St}_{1} \times \mathrm{St}_{2} ; \\
\text { - } & \frac{1}{1-q x_{1} x_{2}^{-1}} & \text { si } \pi=\mathrm{St}_{1} \times \mathrm{St}_{3} \times \mathrm{St}_{1} ; \\
\text { - } \frac{1-q x_{2}}{1-q x_{1} x_{2}^{-1}} \frac{1}{1-q x_{2}} & \text { si } \pi=\mathrm{St}_{5} ; \\
& \text { si } \pi=\mathrm{St}_{1} \times \xi \mathrm{St}_{3} \times \xi \mathrm{St}_{1} .
\end{array}
$$

Démonstration. - Écrivons :

$$
S(f)\left(X_{1}, \ldots, X_{5}\right)=\sum_{\boldsymbol{m}=\left(m_{1}, \ldots, m_{5}\right) \in \mathbb{Z}^{5}} c_{\boldsymbol{m}} X_{1}^{m_{1}} \cdots X_{5}^{m_{5}}
$$

Alors, par définition,

$$
p(S(f))\left(X_{1}, X_{2}\right)=\sum_{\boldsymbol{m} \in \mathbb{Z}^{5}} c_{\boldsymbol{m}} X_{1}^{m_{1}-m_{5}} X_{2}^{m_{2}-m_{4}}=\sum_{\mathbf{n}=\left(n_{1}, n_{2}\right) \in \mathbb{Z}^{2}} d_{\boldsymbol{n}} X_{1}^{n_{1}} X_{2}^{n_{2}}
$$

où

$$
d_{\boldsymbol{n}}=\sum_{\substack{\boldsymbol{m} \in \mathbb{Z}^{5} \\ m_{1}-m_{5}=n_{1} \\ m_{2}-m_{4}=n_{2}}} c_{\boldsymbol{m}}
$$

3.3.1. Cas symétriques. - Définissons $\mathcal{N}_{\pi}$ et $q_{\pi, \boldsymbol{n}}$ de façon suivante :

$$
\begin{aligned}
& \text { • } \begin{cases}\mathcal{N}_{\pi}=\left\{\boldsymbol{n}=\left(n_{1}, n_{2}\right) \in \mathbb{Z}^{2} ; n_{1}=n_{2}=0\right\} & \text { si } \pi=\mathrm{St}_{1} \times \cdots \times \mathrm{St}_{1} ; \\
q_{\pi, \boldsymbol{n}}=1 & \text { si } \pi=\mathrm{St}_{2} \times \mathrm{St}_{1} \times \mathrm{St}_{2} ;\end{cases} \\
& \text { • }\left\{\begin{array}{l}
\mathcal{N}_{\pi}=\left\{\boldsymbol{n}=\left(n_{1}, n_{2}\right) \in \mathbb{Z}^{2} ; n_{1}=n_{2}>0\right\} \\
q_{\pi, \boldsymbol{n}}=-q^{-n_{1}}
\end{array}\right. \\
& \text { • }\left\{\begin{array}{l}
\mathcal{N}_{\pi}=\left\{\boldsymbol{n}=\left(n_{1}, n_{2}\right) \in \mathbb{Z}^{2} ; n_{1}>0, n_{2}=0\right\} \\
q_{\pi, \boldsymbol{n}}=-q^{-n_{1}}
\end{array}\right. \\
& \text { - }\left\{\begin{array}{l}
\mathcal{N}_{\pi}=\left\{\boldsymbol{n}=\left(n_{1}, n_{2}\right) \in \mathbb{Z}^{2} ; n_{1}>0, n_{1}+n_{2}>\mathrm{St}_{3} \times \mathrm{St}_{1} ;\right. \\
q_{\pi, \boldsymbol{n}}=q^{-2 n_{1}-n_{2}}
\end{array}\right.
\end{aligned}
$$

On peut alors écrire (si $\pi$ est symétrique, rappelons-le) :

$$
\operatorname{tr}_{\theta-c} \pi(f)=\sum_{\boldsymbol{n} \in \mathcal{N}_{\pi}} d_{\boldsymbol{n}} q_{\pi, \boldsymbol{n}}
$$

D'autre part, on a la formule d'orthogonalité suivante :

$$
\int_{\substack{\left|x_{1}\right|=\delta_{1} \\\left|x_{2}\right|=\delta_{2}}} x_{1}^{i} x_{2}^{j} \cdot x_{1}^{i^{\prime}} x_{2}^{j^{\prime}} d^{\times} x_{1} d^{\times} x_{2}= \begin{cases}1 & \text { si } i+i^{\prime}=j+j^{\prime}=0 \\ 0 & \text { sinon }\end{cases}
$$


cela pour tous $\delta_{1}, \delta_{2}$ positifs. On déduit des formules $3.19,3.20$ :

$$
\begin{aligned}
\operatorname{tr}_{\theta-c} \pi(f) & =\sum_{\boldsymbol{n} \in \mathcal{N}_{\pi}} \int_{\substack{\left|x_{1}\right|=\delta_{1} \\
\left|x_{2}\right|=\delta_{2}}} p(S(f))\left(x_{1}, x_{2}\right) q_{\pi, \boldsymbol{n}} x_{1}^{-n_{1}} x_{2}^{-n_{2}} d^{\times} x_{1} d^{\times} x_{2} \\
& =\int_{\substack{\left|x_{1}\right|=\delta_{1} \\
\left|x_{2}\right|=\delta_{2}}} p(S(f))\left(x_{1}, x_{2}\right)\left(\sum_{\boldsymbol{n} \in \mathcal{N}_{\pi}} q_{\pi, \boldsymbol{n}} x_{1}^{-n_{1}} x_{2}^{-n_{2}}\right) d^{\times} x_{1} d^{\times} x_{2}
\end{aligned}
$$

pourvu que cette expression converge.

Or, on montre aisément que la somme $\sum_{\boldsymbol{n} \in \mathcal{N}_{\pi}} q_{\pi, \boldsymbol{n}} x_{1}^{-n_{1}} x_{2}^{-n_{2}}$ converge uniformément sur $\widehat{T}_{u}=\left\{\left(x_{1}, x_{2}\right) ;\left|x_{1}\right|=\left|x_{2}\right|=1\right\}$ vers

$$
\begin{aligned}
& \text { - } 1=Q_{\pi}\left(x_{1}, x_{2}\right) \text { si } \pi=\mathrm{St}_{1} \times \mathrm{St}_{1} \times \mathrm{St}_{1} \times \mathrm{St}_{1} \times \mathrm{St}_{1} \text {; } \\
& \text { - }-\frac{q^{-1} x_{1}^{-1} x_{2}^{-1}}{1-q^{-1} x_{1}^{-1} x_{2}^{-1}}=Q_{\pi}\left(x_{1}, x_{2}^{-1}\right) \text { si } \pi=\mathrm{St}_{2} \times \mathrm{St}_{1} \times \mathrm{St}_{2} \text {; } \\
& \text { - }-\frac{q^{-1} x_{1}^{-1}}{1-q^{-1} x_{1}^{-1}} \quad=Q_{\pi}\left(x_{2}, x_{1}\right) \text { si } \pi=\mathrm{St}_{1} \times \mathrm{St}_{3} \times \mathrm{St}_{1} \text {; } \\
& \text { - } \frac{q^{-1} x_{1}^{-1} x_{2}}{1-q^{-1} x_{1}^{-1} x_{2}} \frac{q^{-1} x_{2}^{-1}}{1-q^{-1} x_{2}^{-1}}=Q_{\pi}\left(x_{1}, x_{2}\right) \quad \text { si } \pi=\mathrm{St}_{5} \text {. }
\end{aligned}
$$

Pour conclure, il suffit alors d'utiliser l'invariance de $p(S(f))$ sous l'action du groupe de Weyl $W\left(C_{2}\right)$ :

$$
\begin{aligned}
\int_{\widehat{T}_{u}} p(S(f))\left(x_{1}, x_{2}\right) Q_{\pi}\left(x_{1}, x_{2}\right) d^{\times} x_{1} d^{\times} x_{2} & =\int_{\widehat{T}_{u}} p(S(f))^{\sigma}\left(x_{1}, x_{2}\right) Q_{\pi}\left(x_{1}, x_{2}\right) d^{\times} x_{1} d^{\times} x_{2} \\
& =\int_{\widehat{T}_{u}} p(S(f))\left(x_{1}, x_{2}\right) Q_{\pi}^{\sigma}\left(x_{1}, x_{2}\right) d^{\times} x_{1} d^{\times} x_{2}
\end{aligned}
$$

cela pour tout $\sigma \in W\left(C_{2}\right)$.

3.3.2. Cas $\pi=\mathrm{St}_{1} \times \xi \mathrm{St}_{3} \times \xi \mathrm{St}_{1}$. - Posons :

$$
\begin{array}{ll}
\mathcal{N}_{\pi}=\left\{\boldsymbol{n}=\left(n_{1}, n_{2}\right) \in \mathbb{Z}^{2} ; n_{1}>0, n_{2}=0\right\} & q_{\pi, \boldsymbol{n}}=-(-q)^{-n_{1}} \\
\mathcal{N}_{\pi}^{\prime}=\left\{\boldsymbol{n}=\left(n_{1}, n_{2}\right) \in \mathbb{Z}^{2} ; n_{1}>n_{2} \geqslant 0\right\} & q_{\pi, \boldsymbol{n}}^{\prime}=2(-q)^{-n_{1}}(-1)^{-n-2}
\end{array}
$$

D'après la proposition 3.17 , on a l'égalité :

$$
\operatorname{tr}_{\theta-c} \pi(f)=\sum_{\boldsymbol{n} \in \mathcal{N}_{\pi}} d_{\boldsymbol{n}} q_{\pi, \boldsymbol{n}}+\sum_{\boldsymbol{n} \in \mathcal{N}_{\pi}^{\prime}} d_{\boldsymbol{n}} q_{\pi, \boldsymbol{n}}^{\prime}
$$


On déduit des formules $3.20,3.21$ :

$$
\begin{aligned}
\operatorname{tr}_{\theta-c} \pi(f)= & \sum_{\boldsymbol{n} \in \mathcal{N}_{\pi}} \int_{\substack{\left|x_{1}\right|=\delta_{1} \\
\left|x_{2}\right|=\delta_{2}}} p(S(f))\left(x_{1}, x_{2}\right) q_{\pi, \boldsymbol{n}} x_{1}^{-n_{1}} x_{2}^{-n_{2}} d^{\times} x_{1} d^{\times} x_{2}+ \\
& +\sum_{\boldsymbol{n} \in \mathcal{N}_{\pi}^{\prime}} \int_{\substack{\left|x_{1}\right|=\delta_{1} \\
\left|x_{2}\right|=\delta_{2}}} p(S(f))\left(x_{1}, x_{2}\right) q_{\pi, \boldsymbol{n}}^{\prime} x_{1}^{-n_{1}} x_{2}^{-n_{2}} d^{\times} x_{1} d^{\times} x_{2} \\
= & \int_{\substack{\left|x_{1}\right|=\delta_{1} \\
\left|x_{2}\right|=\delta_{2}}} p(S(f))\left(x_{1}, x_{2}\right) \\
& \cdot\left(\sum_{\boldsymbol{n} \in \mathcal{N}_{\pi}} q_{\pi, \boldsymbol{n}} x_{1}^{-n_{1}} x_{2}^{-n_{2}}+\sum_{\boldsymbol{n} \in \mathcal{N}_{\pi}^{\prime}} q_{\pi, \boldsymbol{n}}^{\prime} x_{1}^{-n_{1}} x_{2}^{-n_{2}}\right) d^{\times} x_{1} d^{\times} x_{2}
\end{aligned}
$$

pourvu que cette expression converge.

Choisissons $\delta_{1}=1, \delta_{2}=\delta>1$ alors, on vérifie aisément que la somme $\sum_{\boldsymbol{n} \in \mathcal{N}_{\pi}} q_{\pi, \boldsymbol{n}} x_{1}^{-n_{1}} x_{2}^{-n_{2}}+\sum_{\boldsymbol{n} \in \mathcal{N}_{\pi}^{\prime}} q_{\pi, \boldsymbol{n}}^{\prime} x_{1}^{-n_{1}} x_{2}^{-n_{2}}$ converge uniformément sur $\left\{\left(x_{1}, x_{2}\right) \in\right.$ $\left.\mathbb{C}^{2} ;\left|x_{1}\right|=1,\left|x_{2}\right|=\delta\right\}$ vers

$$
\begin{aligned}
P\left(x_{1}, x_{2}\right) & =\frac{q^{-1} x_{1}^{-1}}{1+q^{-1} x_{1}^{-1}}-2 \frac{1}{1+x_{2}^{-1}}\left(\frac{q^{-1} x_{1}^{-1}}{1+q^{-1} x_{1}^{-1}}+\frac{q^{-1} x_{1}^{-1} x_{2}^{-1}}{1+q^{-1} x_{1}^{-1}}\right) \\
& =\frac{q^{-1} x_{1}^{-1}}{1+q^{-1} x_{1}^{-1}}-2 \frac{q^{-1} x_{1}^{-1}}{\left(1+q^{-1} x_{1}^{-1}\right)\left(1-q^{-1} x_{1}^{-1} x_{2}^{-1}\right)} \\
& =-\frac{q^{-1} x_{1}^{-1}}{1+q^{-1} x_{1}^{-1}} \frac{1+q^{-1} x_{1}^{-1} x_{2}^{-1}}{1-q^{-1} x_{1}^{-1} x_{2}^{-1}}
\end{aligned}
$$

Or on remarquera que $P$ est holomorphe au voisinage de $\left|x_{2}\right|=1$. On en conclut :

$$
\operatorname{tr}_{\theta-c} \pi(f)=\int_{\widehat{T}_{u}} p(S(f))\left(x_{1}, x_{2}\right) P\left(x_{1}, x_{2}\right) d^{\times} x_{1} d^{\times} x_{2}
$$

Enfin, il suffit de faire un calcul élémentaire pour constater que :

$$
\frac{1}{2}\left(P\left(x_{1}, x_{2}\right)+P\left(x_{1}, x_{2}^{-1}\right)\right)=Q_{\pi}\left(x_{1}, x_{2}\right)
$$

Par suite, il suffit d'utiliser l'invariance de $p(S(f))$ sous l'action de $W\left(C_{n}\right)$ pour conclure.

Le théorème 3.18 est complètement démontré.

Remarque 3.22. - Lorsque $\pi=\mathrm{St}_{1} \times \xi \mathrm{St}_{3} \times \xi \mathrm{St}_{1}$, on peut vérifier que :

On obtient :

$$
Q_{\pi}\left(x_{1}, x_{2}\right)=\frac{1}{1-q x_{1} x_{2}^{-1}} \frac{1}{1+x_{2}}+\frac{1}{1-q x_{1} x_{2}} \frac{1}{1+x_{2}^{-1}}
$$

$\operatorname{tr}_{\theta-c} \pi=\frac{2}{\left|W\left(C_{2}\right)\right|} \int_{\widehat{T}_{u}} p(S(f))\left(x_{1}, x_{2}\right)\left(\sum_{\sigma \in W\left(C_{2}\right)}\left(\frac{1}{1-q x_{1} x_{2}^{-1}} \frac{1}{1+x_{2}}\right)^{\sigma}\right) d^{\times} x_{1} d^{\times} x_{2}$ 


\section{CHAPITRE 4}

\section{INTÉGRALES ORBITALES UNIPOTENTES STABLES \\ SUR $\operatorname{Sp}(4)$}

\subsection{Formule de Rao}

Soit $\mathbf{H}$ un groupe réductif, connexe, défini sur $F$, et soit $\mathfrak{H}$ son algèbre de Lie. Soit $X_{0}$ un élément nilpotent de $\mathfrak{H}(F)$. D'après le théorème de JacobsonMorozov, il existe un $\mathfrak{s l}_{2}$-triplet $\left\{X_{0}, H_{0}, Y_{0}\right\}$ associé à $X_{0}$. Pour $m \in \mathbb{Z}$, posons : $\mathfrak{H}_{m}=\left\{X \in \mathfrak{H} ;\left[H_{0}, X\right]=m X\right\}$. Posons :

$$
\begin{aligned}
& \mathfrak{P}=\oplus_{m \geqslant 0} \mathfrak{H}_{m} \\
& \mathfrak{N}_{\mathfrak{n}}=\oplus_{m>n} \mathfrak{H}_{m}
\end{aligned}
$$

Alors $\mathfrak{P}$ est une sous-algèbre parabolique, de radical nilpotent $\mathfrak{N}_{0}$. Soient $\mathbf{P}, \mathbf{N}$ les sous-groupes de $\mathbf{G}$ correspondant à ces algèbres de Lie. Notons $M$ le centralisateur de $H_{0}$ dans $H$, on a alors l'égalité : $P=M N$. Il existe un sous-groupe compact maximal $K$ de $H$ tel que $H=K P$. Posons : $V\left(X_{0}\right)=\left\{\operatorname{Ad} m\left(X_{0}\right) \mid m \in M\right\}$. Alors la $H$-orbite de $X_{0}$ est égale à $\operatorname{Ad} K\left(V\left(X_{0}\right)+\mathfrak{N}_{2}\right)$. Soient $Z_{1}, Z_{2}, \ldots, Z_{r}$ et $Z_{1}^{\prime}, Z_{2}^{\prime}, \ldots, Z_{r}^{\prime}$ deux bases de $\mathfrak{H}_{1}$ et $\mathfrak{H}_{-1}$ telles que $B\left(Z_{i}, Z_{j}^{\prime}\right)=\delta_{i j}$, où $B($, ) est la forme de Killing sur $\mathfrak{H}$. Pour $X \in \mathfrak{H}_{2}$, on pose : $\left[X, Z_{j}^{\prime}\right]=\sum_{i} c_{i j} Z_{i}$ et $\phi(X)=\left|\operatorname{det}\left(c_{i j}\right)\right|^{1 / 2}$.

Soit $f \in C_{c}^{\infty}(H)$, définissons $f_{K}$ par la formule : $f_{K}(X)=\int_{K} f(\operatorname{Ad} k(X)) d k$. Les espaces vectoriels en question seront tous munis de mesures euclidiennes. Posons : $u=\exp \left(X_{0}\right)$. Notons $\mathcal{O}_{u}$ l'orbite de $u$. Le résultat suivant est notre outil pour calculer des intégrales orbitales unipotentes :

Proposition 4.1 (Rao). - Il existe une constante c telle que pour toute fonction $f \in C_{c}^{\infty}(H)$ on ait l'égalité :

$$
\int_{\mathcal{O}_{u}} f=c \int_{V\left(X_{0}\right)+\mathfrak{n}_{2}} \phi(X) f_{K}(X+Z) d X d Z
$$

Remarque 4.3. - On peut normaliser les mesures en question de telle sorte que l'égalité ci-dessus ait lieu pour $c=1$, c'est ce que nous ferons. 


\subsection{Distributions stablement invariantes à support unipotent}

Dorénavant, $\mathbf{H}$ désignera le groupe $\mathbf{S p}(4)$. Plus précisément :

$$
\mathbf{H}=\left\{M \in \mathbf{S L}(4) ;{ }^{t} M J M^{-1}=J\right\}
$$

où

$$
J=\left(\begin{array}{cccc}
0 & 0 & 0 & 1 \\
0 & 0 & 1 & 0 \\
0 & -1 & 0 & 0 \\
-1 & 0 & 0 & 0
\end{array}\right)
$$

Supposons que $q$ est impair et fixons un élément $\varepsilon \in \mathcal{O}_{F}$ dont la réduction dans $\mathbb{F}_{q}$ n'est pas un carré de sorte que $\{1, \varepsilon, \varpi, \varepsilon \varpi\}$ forment un système de représentants de $F^{\times} /\left(F^{\times}\right)^{2}$. Soit $Q$ une forme quadratique non dégénérée sur $F$, nous notons $i(Q)$ son invariant de Hasse et $\delta(Q)$ son discriminant usuel multiplié par $(-1)^{[d(Q) / 2]}$ modulo $\left(F^{\times}\right)^{2}$, où $d(Q)$ est le rang de $Q$. Ainsi, le discriminant d'une forme quadratique $a x^{2}+b y^{2}$ est égal à $-a b \bmod \left(F^{\times}\right)^{2}$. Le couple $(\delta(Q), i(Q))$ détermine la classe d'équivalence de $Q$. Avec cette normalisation il y a 7 classes de conjugaison de formes quadratiques non dégénérées de rang 2 sur $F$ correspondant à $(\delta, i)=(1,1),(\varepsilon, 1),(\varpi, 1),(\varepsilon \varpi, 1),(\varepsilon,-1),(\varpi,-1)$ et $(\varepsilon \varpi,-1)$.

On rappelle la liste des orbites unipotentes du groupe $H=\mathbf{H}(F)$ :

- Les orbites régulières, celles associées à la partition (4);

- Les orbites sous-régulières, celles associées à la partition $(2,2)$. Elles sont paramétrées par les classes d'équivalence des formes quadratiques non dégénérées de rang 2 sur $F$. On notera $\mathcal{O}_{\delta, i}$ l'orbite unipotente sous-régulière correspondant à un couple $(\delta, i)$ correspondant à la classe considérée.

- Les orbites 2-régulières, celles associées à la partition $(2,1,1)$. Celles-ci n'interviennent pas dans nos calculs;

- L'orbite triviale, notée $\mathcal{O}_{1}$.

Pour $\mathcal{O}$ une orbite, on note $I_{\mathcal{O}}$ la distribution invariante obtenue par l'intégration sur $\mathcal{O}$. D'après [Wa2], l'espace des distributions unipotentes stablement invariantes sur $H$ admet une base donnée par :

(1) $I_{1}=\sum_{\mathcal{O}}$ régulière $I_{\mathcal{O}}$

(ce qui correspond à $\iota=\{\lambda=(4)\}$ )

(2) $I_{2}=I_{\mathcal{O}_{\varpi, 1}}+I_{\mathcal{O}_{\varepsilon \varpi, 1}}+I_{\mathcal{O}_{\varpi,-1}}+I_{\mathcal{O}_{\varepsilon \varpi,-1}}$

(ce qui correspond à $\iota=\{\lambda=(2,2), \tau=0, \delta=1\}$ )

(3) $I_{3}=I_{\mathcal{O}_{1,1}}+I_{\mathcal{O}_{\varepsilon, 1}}+I_{\mathcal{O}_{\varepsilon,-1}}$

(ce qui correspond à $\iota=\{\lambda=(2,2), \tau=0, \delta=0\}$ )

(4) $I_{4}=I_{\mathcal{O}_{1,1}}-I_{\mathcal{O}_{\varepsilon, 1}}-I_{\mathcal{O}_{\varepsilon,-1}}$

(ce qui correspond à $\iota=\{\lambda=(2,2), \tau=1, \delta=0\}$ )

(5) $I_{5}=$ l'évaluation en l'élément neutre

(ce qui correspond à $\iota=\{\lambda=(1,1,1,1)\}$ ) 
(6) $I_{6}=I_{\mathcal{O}_{\varpi, 1}}-I_{\mathcal{O}_{\varepsilon \varpi, 1}}+I_{\mathcal{O}_{\varpi,-1}}-I_{\mathcal{O}_{\varepsilon \varpi,-1}}$

(ce qui correspond à $\iota=\{\lambda=(2,2), \tau=1, \delta=1\}$ )

Voir [Wa2] pour les définitions des $\iota, \lambda, \tau, \delta$.

Remarquons que l'on a l'égalité :

$$
I_{2}+I_{3}=\sum_{\mathcal{O} \text { sous-régulière }} I_{\mathcal{O}}
$$

\subsection{Formule de Macdonald}

Posons $K=\mathbf{H}\left(\mathcal{O}_{F}\right)$. On définit $\mathcal{H}(H)$ comme en 2.1. L'isomorphisme de Satake, noté par $S$, identifie $\mathcal{H}(H)$ à $\mathbb{C}\left[X_{1}^{ \pm 1}, X_{2}^{ \pm}\right]^{W\left(C_{2}\right)}$, l'algèbre de séries de Laurent en deux variables, invariantes sous l'action du groupe de Weyl de $\mathbf{H}$. Nous suivons [As1] pour la construction explicite de cet isomorphisme.

Soient $m_{1} \geqslant m_{2}$ deux entiers naturels. On pose :

$$
Q_{m_{1}, m_{2}}\left(q^{-1}\right)= \begin{cases}\left(1+q^{-1}\right)^{2}\left(1+q^{-2}\right) & \text { si } m_{1}=m_{2}=0 ; \\ 1+q^{-1} & \text { si } m_{1}>0, m_{2}=0 \text { ou si } m_{1}=m_{2}>0 ; \\ 1 & \text { sinon. }\end{cases}
$$

Posons $Q\left(q^{-1}\right)=Q_{0,0}\left(q^{-1}\right)$. Définissons la fonction $\mathbf{c}_{\mathbf{H}}$ de Harish-Chandra par la formule :

$$
\mathbf{c}_{\mathbf{H}}\left(x_{1}, x_{2}\right)=\frac{1-q^{-1} x_{1}^{-1} x_{2}}{1-x_{1}^{-1} x_{2}} \frac{1-q^{-1} x_{1}^{-1} x_{2}^{-1}}{1-x_{1}^{-1} x_{2}^{-1}} \frac{1-q^{-1} x_{1}^{-1}}{1-x_{1}^{-1}} \frac{1-q^{-1} x_{2}^{-1}}{1-x_{2}^{-1}}
$$

On note $f_{m_{1}, m_{2}}$ la fonction caractéristique de $K \operatorname{diag}\left(\varpi^{m_{1}}, \varpi^{m_{2}}, \varpi^{-m_{2}}, \varpi^{-m_{1}}\right) K$ qui est un élément de $\mathcal{H}(H)$.

Proposition 4.4. - Pour tous $m_{1} \geqslant m_{2} \geqslant 0$ entiers, la transformée de Satake de $f_{m_{1}, m_{2}}$ est donnée par :

$$
S\left(f_{m_{1}, m_{2}}\right)\left(X_{1}, X_{2}\right)=\frac{q^{2 m_{1}+m_{2}}}{Q_{m_{1}, m_{2}}\left(q^{-1}\right)} \sum_{\sigma \in W(H)}\left[\mathbf{c}_{\mathbf{H}}\left(X_{1}, X_{2}\right) X_{1}^{m_{1}} X_{2}^{m_{2}}\right]^{\sigma}
$$

Cette formule, combinée avec la décomposition de Cartan, calcule $S$.

Il est évident que $\mathcal{H}(H)$ est un sous-espace de l'espace $L^{2}(H)$. On note $\langle,\rangle_{L^{2}}$ le produit scalaire usuel. Le résultat suivant, qui est un cas particulier de la formule de Plancherel, est dû à Macdonald :

ThÉORÈme 4.5 (Formule de Macdonald). - Pour tous $f, g \in \mathcal{H}(H)$, on a l'égalité :

$\langle f, g\rangle_{L^{2}}=\frac{Q\left(q^{-1}\right)}{|W(H)|} \int_{\widehat{T}_{u}} S(f)\left(x_{1}, x_{2}\right) S(g)\left(x_{1}, x_{2}\right) \mathbf{c}_{\mathbf{H}}\left(x_{1}, x_{2}\right)^{-1} \mathbf{c}_{\mathbf{H}}\left(x_{1}^{-1}, x_{2}^{-1}\right)^{-1} d^{\times} x_{1} d^{\times} x_{2}$

Si on prend $g=\mathbf{1}_{K}$ la fonction caractéristique de $K$, alors pour tout $f \in \mathcal{H}(H)$ on a : $\langle f, g\rangle_{L^{2}}=f(1)$. On obtient : 
Corollaire 4.6. - Pour tout $f \in \mathcal{H}(H)$ on a l'égalité :

$$
f(1)=\frac{Q\left(q^{-1}\right)}{|W(H)|} \int_{\widehat{T}_{u}} S(f)\left(x_{1}, x_{2}\right) \mathbf{c}_{\mathbf{H}}\left(x_{1}, x_{2}\right)^{-1} \mathbf{c}_{\mathbf{H}}\left(x_{1}^{-1}, x_{2}^{-1}\right)^{-1} d^{\times} x_{1} d^{\times} x_{2}
$$

REMARQue 4.7. - Les résultats de ce paragraphe ont été obtenus en toute généralité par Macdonald dans [Mac], nous pouvons appliquer ces formules pour d'autres groupes autres que $\mathbf{S p}(4)$ si nécessaire.

\subsection{Transformation de Satake}

4.4.1. Annulation. — L'assertion suivante découle immédiatement de [As1]:

Proposition 4.8. - Pour tout $f \in \mathcal{H}(H)$, on a $I_{6}(f)=0$

4.4.2. Autres cas. - Nous commençons par le résultat suivant :

Proposition 4.9. - Pour tout $f \in \mathcal{H}(H)$, on a :

$$
\begin{aligned}
I_{1}(f) & =\int_{\widehat{T}_{u}} S(f)\left(x_{1}, x_{2}\right) d^{\times} x_{1} d^{\times} x_{2} \\
I_{2}(f)+I_{3}(f) & =\frac{1+q^{-1}}{2} \int_{\widehat{T}_{u}} S(f)\left(x_{1}, x_{2}\right) \frac{1-x_{1}^{-1} x_{2}}{1-q^{-1} x_{1}^{-1} x_{2}} \frac{1-x_{1} x_{2}^{-1}}{1-q^{-1} x_{1} x_{2}^{-1}} d^{\times} x_{1} d^{\times} x_{2} \\
I_{3}(f)+I_{4}(f) & =\frac{1+q^{-1}}{2} \int_{\widehat{T}_{u}} S(f)\left(x_{1}, x_{2}\right) \frac{1-x_{1}}{1-q^{-1} x_{1}} \frac{1-x_{1}^{-1}}{1-q^{-1} x_{1}^{-1}} d^{\times} x_{1} d^{\times} x_{2} \\
I_{5}(f) & =\frac{Q\left(q^{-1}\right)}{|W|} \int_{\widehat{T}_{u}} S(f)\left(x_{1}, x_{2}\right) \mathbf{c}_{\mathbf{H}}\left(x_{1}, x_{2}\right)^{-1} \mathbf{c}_{\mathbf{H}}\left(x_{1}^{-1}, x_{2}^{-1}\right)^{-1} d^{\times} x_{1} d^{\times} x_{2}
\end{aligned}
$$

Démonstration. - Voir [As2, Prop. 3.1], mais avec la réserve suivante sur $I_{3}(f)+$ $I_{4}(f)=2 I_{\mathcal{O}_{1,1}}(f)$ ( $c f$. paragraphe 4.2 ). Dans [As2], l'auteur a donné la formule :

$$
I_{\mathcal{O}_{1,1}}(f)=\frac{1+q^{-1}}{2} \int_{\widehat{T}_{u}} S(f)\left(x_{1}, x_{2}\right) \frac{1-x_{1}}{1-q^{-1} x_{1}} \frac{1-x_{1}^{-1}}{1-q^{-1} x_{1}^{-1}} d^{\times} x_{1} d^{\times} x_{2}
$$

En fait, dans cette formule, il fallait changer $\frac{1+q^{-1}}{2}$ en $\frac{1+q^{-1}}{4}$. Pour s'en convaincre, il suffit de vérifier pour $f=\mathbf{1}_{K}$. D'après [As2, Th. 2.3.7], $I_{\mathcal{O}_{1,1}}(f)\left(\mathbf{1}_{K}\right)=\frac{1}{2}$. D'autre part, un simple calcul des résidus montre que

$$
\frac{1+q^{-1}}{2} \int_{\widehat{T}_{u}} \frac{1-x_{1}}{1-q^{-1} x_{1}} \frac{1-x_{1}^{-1}}{1-q^{-1} x_{1}^{-1}} d^{\times} x_{1} d^{\times} x_{2}=1 .
$$


4.4.3. Calcul de $I_{4}$. - Les résultats de ce paragraphe peuvent être facilement déduits de [As2]. Cependant, il se trouve que dans notre situation, les calculs soient un peu moins compliqués, nous allons donc donner quelques preuves.

Nous suivons [As2] pour la description des données relatives aux orbites sousrégulières. Considérons le $\mathfrak{s l}_{2}$-triplet suivant :

$$
X_{0}=\left(\begin{array}{cccc}
0 & 0 & 0 & 1 \\
0 & 0 & 1 & 0 \\
0 & 0 & 0 & 0 \\
0 & 0 & 0 & 0
\end{array}\right), \quad H_{0}=\left(\begin{array}{cccc}
1 & 0 & 0 & 0 \\
0 & 1 & 0 & 0 \\
0 & 0 & -1 & 0 \\
0 & 0 & 0 & -1
\end{array}\right), \quad Y_{0}=\left(\begin{array}{cccc}
0 & 0 & 0 & 0 \\
0 & 0 & 0 & 0 \\
0 & 1 & 0 & 0 \\
1 & 0 & 0 & 0
\end{array}\right)
$$

Alors, on a :

$$
\begin{gathered}
\mathfrak{H}_{-1}=\mathfrak{H}_{1}=0, \quad \mathfrak{H}_{2}=\left\{\left(\begin{array}{cccc}
0 & 0 & y & x \\
0 & 0 & z & y \\
0 & 0 & 0 & 0 \\
0 & 0 & 0 & 0
\end{array}\right) ; x, y, z \in F\right\} \\
M=\left\{\left(\begin{array}{cc}
A & 0 \\
0 & { }^{t} A^{-1}
\end{array}\right) ; A \in \mathbf{G} \mathbf{L}(2, F)\right\}
\end{gathered}
$$

Identifions $\mathfrak{H}_{2}$ à l'espace des formes quadratiques de rang $2, x X^{2}+2 y X Y+z Y^{2}$, $x, y, z \in F$. L'action de $M$ est l'action de $\mathbf{G L}(2, F)$ sur les formes quadratiques. Les orbites ouvertes de $M$ dans $\mathfrak{H}_{2}$, qui sont en bijection avec l'ensemble des orbites unipotentes sous-régulières, s'identifient donc aux classes d'équivalence des formes quadratiques non dégénérées de rang 2 sur $F$. On a vu qu'elles sont en nombre de 7 , paramétrées par les couples $(\delta, i)$. Explicitons les ensembles $V$ qui interviennent dans la formule de Rao. Soit $V_{\delta, i}$ l'ensemble relatif à l'orbite $\mathcal{O}_{\delta, i}$.

Lemme 4.10 (Assem). - Posons : $X=\left(\begin{array}{cccc}0 & 0 & y & x \\ 0 & 0 & z & y \\ 0 & 0 & 0 & 0 \\ 0 & 0 & 0 & 0\end{array}\right)$. Alors,

$$
\begin{aligned}
V_{1,1} & =\left\{X \in \mathfrak{H}_{2} ; y^{2}-x z \in\left(F^{x}\right)^{2}\right\} \\
V_{\varepsilon, 1} & =\left\{X \in \mathfrak{H}_{2} ; y^{2}-x z \in \varepsilon\left(F^{x}\right)^{2}, \operatorname{val}(x) \text { paire }\right\} \\
V_{\varepsilon,-1} & =\left\{X \in \mathfrak{H}_{2} ; y^{2}-x z \in \varepsilon\left(F^{x}\right)^{2}, \operatorname{val}(x) \text { impaire }\right\}
\end{aligned}
$$

Posons :

$$
\begin{aligned}
V^{+} & =\left\{X \in \mathfrak{H}_{2} ; y^{2}-x z \in\left(F^{x}\right)^{2}\right\} \\
V^{-} & =\left\{X \in \mathfrak{H}_{2} ; y^{2}-x z \in \varepsilon\left(F^{x}\right)^{2}\right\}
\end{aligned}
$$

Puisque $\mathfrak{H}_{-1}=\mathfrak{H}_{1}=0$, la fonction $\phi$ dans la formule de Rao est égale à 1 , on obtient :

$$
I_{4}(f)=\int_{V+} f_{K}(Z) d Z-\int_{V^{-}} f_{K}(Z) d Z
$$


Maintenant, si $f=f_{m_{1}, m_{2}}$ alors, par définition $f_{m_{1}, m_{2}}$ est invariant par $K$, il vient : (4.11) $I_{4}\left(f_{m_{1}, m_{2}}\right)=\operatorname{mes}\left\{V^{+} \cap \operatorname{supp}\left(f_{m_{1}, m_{2}} \circ \exp \right)\right\}-\operatorname{mes}\left\{V^{-} \cap \operatorname{supp}\left(f_{m_{1}, m_{2}} \circ \exp \right)\right\}$

Les ensembles dans la formule ci-dessus ont été décrits dans [As2] :

LEMME 4.12 (Assem). - On a :

$$
\mathfrak{H}_{2} \cup \operatorname{supp}\left(f_{m_{1}, m_{2}} \circ \exp \right)=\left[\begin{array}{rl}
\bullet & \left\{X \in \mathfrak{H}_{2} ; \min (\operatorname{val}(x), \operatorname{val}(y), \operatorname{val}(z))=-m_{1},\right. \\
& \left.\operatorname{val}\left(y^{2}-x z\right)=-m_{1}-m_{2}\right\} \quad \text { si } m_{1} \geqslant m_{2}>0 ; \\
\bullet & \left\{X \in \mathfrak{H}_{2} ; \min (\operatorname{val}(x), \operatorname{val}(y), \operatorname{val}(z))=-m_{1},\right. \\
& \left.\operatorname{val}\left(y^{2}-x z\right) \geqslant-m_{1}\right\} \quad \text { si } m_{1}>m_{2}=0 ; \\
\bullet & \left\{X \in \mathfrak{H}_{2} ; \min (\operatorname{val}(x), \operatorname{val}(y), \operatorname{val}(z)) \geqslant 0\right\} \\
& \text { si } m_{1}=m_{2}=0 .
\end{array}\right.
$$

Pour $k \in \mathbb{N}$, posons :

$$
\begin{gathered}
N_{k}=\operatorname{mes}\left\{X \in \mathfrak{H}_{2} ; \min (\operatorname{val}(x), \operatorname{val}(y), \operatorname{val}(z))=0, \operatorname{val}\left(y^{2}-x z\right)=k,\right. \\
\left.y^{2}-x z \in\left(F^{x}\right)^{2}\right\} \\
-\operatorname{mes}\left\{X \in \mathfrak{H}_{2} ; \min (\operatorname{val}(x), \operatorname{val}(y), \operatorname{val}(z))=0, \operatorname{val}\left(y^{2}-x z\right)=k,\right. \\
\left.y^{2}-x z \in \varepsilon\left(F^{x}\right)^{2}\right\}
\end{gathered}
$$

LEMme 4.13. - On a $: N_{k}=0$, sauf pour $k=0$ auquel cas $N_{0}=q^{-1}\left(1-q^{-1}\right)$.

Démonstration. - Pour $k$ impair, il est clair que $N_{k}=0$. Soit $k>0$, pair. On a : $N_{k}=N_{k}^{\prime}+N_{k}^{\prime \prime}$ avec :

$N_{k}^{\prime}=\operatorname{mes}\left\{X \in \mathfrak{H}_{2} ; \operatorname{val}(x)=0, \operatorname{val}(y) \geqslant 0, \operatorname{val}(z) \geqslant 0, \operatorname{val}\left(y^{2}-x z\right)=k\right.$,

$$
\begin{gathered}
\left.y^{2}-x z \in\left(F^{x}\right)^{2}\right\} \\
-\operatorname{mes}\left\{X \in \mathfrak{H}_{2} ; \operatorname{val}(x)=0, \operatorname{val}(y) \geqslant 0, \operatorname{val}(z) \geqslant 0, \operatorname{val}\left(y^{2}-x z\right)=k,\right. \\
\left.y^{2}-x z \in \varepsilon\left(F^{x}\right)^{2}\right\} \\
N_{k}^{\prime \prime}=\operatorname{mes}\left\{X \in \mathfrak{H}_{2} ; \operatorname{val}(x) \geqslant 1, \min (\operatorname{val}(x), \operatorname{val}(y), \operatorname{val}(z))=0, \operatorname{val}\left(y^{2}-x z\right)=k,\right. \\
\left.y^{2}-x z \in\left(F^{x}\right)^{2}\right\} \\
-\operatorname{mes}\left\{X \in \mathfrak{H}_{2} ; \operatorname{val}(x) \geqslant 1, \min (\operatorname{val}(x), \operatorname{val}(y), \operatorname{val}(z))=0, \operatorname{val}\left(y^{2}-x z\right)=k,\right. \\
\left.y^{2}-x z \in \varepsilon\left(F^{x}\right)^{2}\right\}
\end{gathered}
$$

Calculons $N_{k}^{\prime}$. Soit $x$ tel que $\operatorname{val}(x)=0$. On pose $: \delta=y^{2}-x z$. Le jacobien du changement de variable $(x, y, z) \mapsto(x, y, \delta)$ est égal à 1 , donc :

$$
\begin{aligned}
N_{k}^{\prime}=\operatorname{mes}\left\{(x, y, \delta) \in F^{3}\right. & \left.; \operatorname{val}(x)=0, \operatorname{val}(\delta)=k, \delta \in\left(F^{x}\right)^{2}\right\} \\
& \quad-\operatorname{mes}\left\{(x, y, \delta) \in F^{3} ; \operatorname{val}(x)=0, \operatorname{val}(\delta)=k, \delta \in \varepsilon\left(F^{x}\right)^{2}\right\}
\end{aligned}
$$

En utilisant le simple changement de variable $\delta \mapsto \varepsilon \delta$, on obtient : $N_{k}^{\prime}=0$.

Calculons $N_{k}^{\prime \prime}$. Soit $x$ tel que $\operatorname{val}(x) \geqslant 1$. Alors la condition $\operatorname{val}\left(y^{2}-x z\right)=k$ implique que $\operatorname{val}(y)>0, \operatorname{donc} \operatorname{val}(z)=0$ parce que $\min (\operatorname{val}(x), \operatorname{val}(y), \operatorname{val}(z))=0$. 
Par des arguments similaires que ci-dessus et en utilisant le changement de variable $(x, y, z) \mapsto\left(\delta=y^{2}-x z, y, z\right)$, on obtient : $N_{k}^{\prime \prime}=0$. D'où : $N_{k}=0$, si $k>0$.

Enfin, calculons $N_{0}$. En réduisant modulo l'idéal maximal de $\mathcal{O}_{F},(x, y, z)=$ $(\bar{x}, \bar{y}, \bar{z}) \bmod \left(\varpi \mathcal{O}_{F}\right)^{3}$, on obtient :

$$
\begin{aligned}
N_{0}=q^{-3} \operatorname{card}\left\{(\bar{x}, \bar{y}, \bar{z}) \in \mathbb{F}_{q} ;(\bar{x}, \bar{y}, \bar{z})\right. & \left.\neq(0,0,0), \bar{y}^{2}-\overline{x z} \in\left(\mathbb{F}_{q}^{\times}\right)^{2}\right\} \\
-q^{-3} \operatorname{card}\{(\bar{x}, \bar{y}, \bar{z}) & \left.\in \mathbb{F}_{q} ;(\bar{x}, \bar{y}, \bar{z}) \neq(0,0,0), \bar{y}^{2}-\overline{x z} \in \bar{\varepsilon}\left(\mathbb{F}_{q}^{\times}\right)^{2}\right\}
\end{aligned}
$$

Un calcul élémentaire montre que $N_{0}=q^{-1}\left(1-q^{-1}\right)$, d'où le lemme.

Proposition 4.14. - Soient $m_{1} \geqslant m_{2} \geqslant 0$, alors $I_{4}\left(f_{m_{1}, m_{2}}\right)$ est égal à

(1) $\frac{q^{-1}\left(1-q^{-1}\right)}{1-q^{-3}}$, si $m_{1}=m_{2}=0$;

(2) $q^{-1}\left(1-q^{-1}\right) q^{3 m_{1}}$, si $m_{1}=m_{2}>0$;

(3) 0 , si $m_{1}>m_{2}$.

Démonstration. - Supposons $m_{1}=m_{2}=0$. Grâce au lemme 4.12, la formule 4.11 s'écrit :

$$
\begin{aligned}
I_{4}\left(f_{m_{1}, m_{2}}\right)= & \operatorname{mes}\left\{X \in \mathfrak{H}_{2} ; \min (\operatorname{val}(x), \operatorname{val}(y), \operatorname{val}(z))=0, y^{2}-x z \in\left(F^{\times}\right)^{2}\right\} \\
& \quad-\operatorname{mes}\left\{X \in \mathfrak{H}_{2} ; \min (\operatorname{val}(x), \operatorname{val}(y), \operatorname{val}(z))=0, y^{2}-x z \in \varepsilon\left(F^{\times}\right)^{2}\right\}
\end{aligned}
$$

Ce que l'on découpe en une somme sur les entiers $k \geqslant 0$ de

$$
\begin{aligned}
\operatorname{mes}\left\{X \in \mathfrak{H}_{2} ; \min (\operatorname{val}(x), \operatorname{val}(y), \operatorname{val}(z))=k, y^{2}-x z \in\left(F^{\times}\right)^{2}\right\} \\
\quad-\operatorname{mes}\left\{X \in \mathfrak{H}_{2} ; \min (\operatorname{val}(x), \operatorname{val}(y), \operatorname{val}(z))=k, y^{2}-x z \in \varepsilon\left(F^{\times}\right)^{2}\right\}
\end{aligned}
$$

Par changement de variable, l'expression ci-dessus vaut $q^{-3 k}$ multiplié par

$$
\begin{aligned}
\operatorname{mes}\left\{X \in \mathfrak{H}_{2} ; \min (\operatorname{val}(x), \operatorname{val}(y), \operatorname{val}(z))=0, y^{2}-x z \in\left(F^{\times}\right)^{2}\right\} \\
-\operatorname{mes}\left\{X \in \mathfrak{H}_{2} ; \min (\operatorname{val}(x), \operatorname{val}(y), \operatorname{val}(z))=0, y^{2}-x z \in \varepsilon\left(F^{\times}\right)^{2}\right\}
\end{aligned}
$$

Et ce dernier terme n'est autre que la somme des $N_{k}$ pour $k$ entier $\geqslant 0$. On obtient l'expression

$$
I_{4}\left(f_{m_{1}, m_{2}}\right)=\sum_{k, k \geqslant 0} q^{-3 k} N_{k}
$$

La première égalité de l'énoncé se déduit alors du lemme 4.13. Les deux autres égalités se démontrent de façon analogue.

4.4.4. Transformation de Satake de $I_{4}$ - - Soit $I$ une distribution sur $\mathcal{H}(H)$. Elle est donc déterminée par les constantes $I\left(f_{m_{1}, m_{2}}\right), m_{1} \geqslant m_{2} \geqslant 0$ entiers. Pour $N \in \mathbb{N}$, posons :

$$
I_{N}=\sum_{m_{1} \leqslant N} I\left(f_{m_{1}, m_{2}}\right)\left\|f_{m_{1}, m_{2}}\right\|^{-2} f_{m_{1}, m_{2}}
$$


où ||.|| désigne la norme $L^{2}$ sur $\mathcal{H}(H)$. Il est clair que :

$$
\left\langle I_{N}, f_{m_{1}, m_{2}}\right\rangle_{L^{2}}= \begin{cases}I\left(f_{m_{1}, m_{2}}\right) & \text { si } m_{1} \leqslant N \\ 0 & \text { sinon }\end{cases}
$$

Donc, pour tout $f \in \mathcal{H}(H)$,

$$
I(f)=\lim _{N \rightarrow \infty}\left\langle I_{N}, f_{m_{1}, m_{2}}\right\rangle_{L^{2}}
$$

En utilisant la formule de Macdonald (voir le théorème 4.5), on obtient :

$$
\begin{aligned}
I(f)=\lim _{N \rightarrow \infty} \frac{Q\left(q^{-1}\right)}{|W(H)|} \int_{\widehat{T}_{u}} S(f)\left(x_{1}, x_{2}\right) S\left(I_{N}\right)\left(x_{1}, x_{2}\right) \mathbf{c}_{\mathbf{H}}\left(x_{1}, x_{2}\right)^{-1} \\
\cdot \mathbf{c}_{\mathbf{H}}\left(x_{1}^{-1}, x_{2}^{-1}\right)^{-1} d^{\times} x_{1} d^{\times} x_{2}
\end{aligned}
$$

On a :

$$
S\left(I_{N}\right)\left(x_{1}, x_{2}\right)=\sum_{m_{1} \leqslant N} I\left(f_{m_{1}, m_{2}}\right)\left\|f_{m_{1}, m_{2}}\right\|^{-2} S\left(f_{m_{1}, m_{2}}\right)\left(x_{1}, x_{2}\right)
$$

Posons :

$$
s\left(I_{N}\right)\left(x_{1}, x_{2}\right)=\sum_{m_{1} \leqslant N} I\left(f_{m_{1}, m_{2}}\right)\left\|f_{m_{1}, m_{2}}\right\|^{-2} \frac{q^{2 m_{1}+m_{2}}}{Q_{m_{1}, m_{2}}\left(q^{-1}\right)} x_{1}^{m_{1}} x_{2}^{m_{2}} \mathbf{c}_{\mathbf{H}}\left(x_{1}, x_{2}\right)
$$

Alors,

$$
S\left(I_{N}\right)\left(x_{1}, x_{2}\right)=\sum_{\sigma \in W(H)} s\left(I_{N}\right)\left(x_{1}, x_{2}\right)^{\sigma}
$$

Par invariance, on a aussi :

$$
\begin{array}{r}
I(f)=\lim _{N \rightarrow \infty} Q\left(q^{-1}\right) \int_{\widehat{T}_{u}} S(f)\left(x_{1}, x_{2}\right) s\left(I_{N}\right)\left(x_{1}, x_{2}\right) \mathbf{c}_{\mathbf{H}}\left(x_{1}, x_{2}\right)^{-1} \\
\cdot \mathbf{c}_{\mathbf{H}}\left(x_{1}^{-1}, x_{2}^{-1}\right)^{-1} d^{\times} x_{1} d^{\times} x_{2} \\
=\lim _{N \rightarrow \infty} \int_{\widehat{T}_{u}} S(f)\left(x_{1}, x_{2}\right) \mathbf{c}_{I, N}\left(x_{1}, x_{2}\right) d^{\times} x_{1} d^{\times} x_{2}
\end{array}
$$

où

$$
\begin{aligned}
& \mathbf{c}_{I, N}\left(x_{1}, x_{2}\right)= Q\left(q^{-1}\right) s\left(I_{N}\right)\left(x_{1}, x_{2}\right) \mathbf{c}_{\mathbf{H}}\left(x_{1}, x_{2}\right)^{-1} \mathbf{c}_{\mathbf{H}}\left(x_{1}^{-1}, x_{2}^{-1}\right)^{-1} \\
&=Q\left(q^{-1}\right) \sum_{m_{1} \leqslant N} I\left(f_{m_{1}, m_{2}}\right)\left\|f_{m_{1}, m_{2}}\right\|^{-2} \frac{q^{2 m_{1}+m_{2}}}{Q_{m_{1}, m_{2}}\left(q^{-1}\right)} x_{1}^{m_{1}} x_{2}^{m_{2}} \\
& \cdot \mathbf{c}_{\mathbf{H}}\left(x_{1}^{-1}, x_{2}^{-1}\right)^{-1}
\end{aligned}
$$

D'après [Mac, Prop. 3.2.15],

$$
\begin{aligned}
\left\|f_{m_{1}, m_{2}}\right\|^{-2} & =\operatorname{mes}\left\{K \operatorname{diag}\left(\varpi^{m_{1}}, \varpi^{m_{2}}, \varpi^{-m_{2}}, \varpi^{-m_{1}}\right) K\right\}^{-1} \\
& =q^{-\left(4 m_{1}+2 m_{2}\right)} \frac{Q_{m_{1}, m_{2}}\left(q^{-1}\right)}{Q\left(q^{-1}\right)}
\end{aligned}
$$


D'où :

$$
\mathbf{c}_{I, N}\left(x_{1}, x_{2}\right)=\sum_{m_{1} \leqslant N} q^{-\left(2 m_{1}+m_{2}\right)} I\left(f_{m_{1}, m_{2}}\right) x_{1}^{m_{1}} x_{2}^{m_{2}} \mathbf{c}_{\mathbf{H}}\left(x_{1}^{-1}, x_{2}^{-1}\right)^{-1}
$$

Pour $I=I_{4}$, la fonction $\mathbf{c}_{I, N}$ converge uniformément sur $\widehat{T}_{u}$ vers la fonction $\mathbf{c}_{4}$ définie par :

$$
\mathbf{c}_{4}\left(x_{1}, x_{2}\right)=q^{-1}\left(1-q^{-1}\right)\left(\frac{1}{1-q^{-3}}+\frac{x_{1} x_{2}}{1-x_{1} x_{2}}\right) \mathbf{c}_{\mathbf{H}}\left(x_{1}^{-1}, x_{2}^{-1}\right)^{-1}
$$

En effet, cela résulte de la proposition 4.14 et ci-dessus. On obtient :

Proposition 4.15. - Pour tout $f \in \mathcal{H}(H)$, on a l'égalité :

$$
I_{4}(f)=\int_{\widehat{T}_{u}} S(f)\left(x_{1}, x_{2}\right) \mathbf{c}_{4}\left(x_{1}, x_{2}\right) d^{\times} x_{1} d^{\times} x_{2}
$$

\subsection{Comparaison entre $\mathbf{G L}(5)$ et $\operatorname{Sp}(4)$}

Soit $P$ l'homomorphisme d'algèbres qui rend commutatif le diagramme suivant :

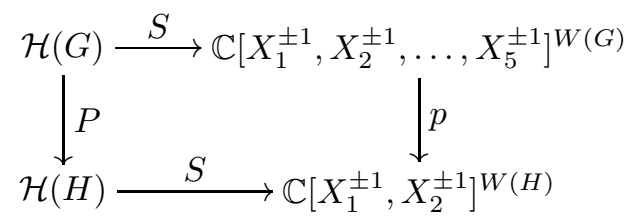

où $S$ désigne l'homomorphisme de Satake et $p$ est l'application $p_{2}$ définie en V.1.0.1.

Soit $\mathcal{I}_{\text {unip }}^{\text {st }}(2)$ l'espace des distributions stables à support unipotent sur $H=S p(4)$. Soit $\mathcal{I}_{\text {unip }}^{\text {st,P }}(2)$ le sous-espace des distributions sur $\mathcal{H}(G)$ déduite de $\mathcal{I}_{\text {unip }}^{\text {st }}(2)$ par composition avec $P$. Soit $\mathcal{T}_{\theta-c}(2)$ la restriction à $\mathcal{H}(G)$ de l'espace engendré par les traces tordues compactes des représentations définies en section 2 .

THÉORÈme 4.16. - Les espaces $\mathcal{I}_{\text {unip }}^{\text {st,P }}(2)$ et $\mathcal{T}_{\theta-c}(2)$ coïncident. C'est un espace de dimension 5 .

C'est un exercice pénible mais élémentaire. L'idée est d'utiliser le théorème 3.18, les propositions 4.9 et 4.15 .

On peut encore préciser ce résultat un peu plus en calculant les matrices de changement de base. On donne ci-dessous le résultat du calcul. Soient $T_{1}, \ldots, T_{5}$ les distributions obtenues à partir des $\operatorname{tr}_{\theta-c} \pi$ pour $\pi=\mathrm{St}_{5}, \mathrm{St}_{1} \times \mathrm{St}_{3} \times \mathrm{St}_{1}, \mathrm{St}_{2} \times \mathrm{St}_{1} \times \mathrm{St}_{2}$, $\mathrm{St}_{1} \times \mathrm{St}_{1} \times \mathrm{St}_{1} \times \mathrm{St}_{1} \times \mathrm{St}_{1}, \mathrm{St}_{1} \times \xi \mathrm{St}_{3} \times \xi \mathrm{St}_{1}$. Pour $i=1, \ldots, 5$, on note par $I_{i}^{P}$ la distribution sur $\mathcal{H}(G)$ obtenue par composition de l'intégrale orbitale unipotente stable $I_{i}$ et $P$. Soit $T I$ la matrice de changement de base de $\left\{T_{i}\right\}$ à $\left\{I_{i}^{P}\right\}: T I=\left(a_{i j}\right)$, avec $I_{j}^{P}=\sum_{i=1}^{5} a_{i j} T_{i}$. Notons $I T$ son inverse, autrement dit la matrice de changement de base de $\left\{I_{i}^{P}\right\}$ à $\left\{T_{i}\right\}$. 
Proposition 4.17. - On a:

$$
\begin{aligned}
T I & =\left(\begin{array}{ccccc}
1 & q & -(q-1) & q & 1 \\
0 & 0 & q-1 & -(q-1) & q^{2}-1 \\
0 & \frac{(q-1)\left(q^{2}+2 q-1\right)}{2} & -\frac{(q-1)\left(q^{2}+2 q-1\right)}{2} & \frac{(q-1)(q+1)^{2}}{2} & \frac{(q-1)\left(q^{2}+q+2\right)}{2} \\
0 & -\frac{(q-1)^{2}(q+1)}{2} & \frac{(q-1)^{2}(q+1)}{2} & -\frac{(q-1)^{2}(q+1)}{2} & \frac{q(q-1)^{2}}{2} \\
0 & 0 & 0 & 0 & \left(q^{2}+q+1\right)(q-1)^{2}
\end{array}\right) \\
I T & =\left(\begin{array}{ccccc}
1 & \frac{-1}{(q-1)} & \frac{-1}{(q-1)} & \frac{-1}{(q-1)} & \frac{1}{(q-1)^{2}} \\
0 & \frac{1}{(q-1)} & 0 & \frac{-2}{(q+1)(q-1)^{2}} & \frac{-1}{(q+1)(q-1)^{2}} \\
0 & \frac{1}{(q-1)} & \frac{1}{(q-1)} & \frac{q^{2}+2 q-1}{(q+1)(q-1)^{2}} & \frac{q+2}{(q+1)(q-1)^{2}} \\
0 & 0 & \frac{1}{(q-1)} & \frac{q^{2}+2 q-1}{(q+1)(q-1)^{2}} & \frac{q^{3}+2 q^{2}+q+1}{(q+1)(q-1)^{2}\left(q^{2}+q+1\right)} \\
0 & 0 & 0 & 0 & \frac{1}{(q-1)^{2}\left(q^{2}+q+1\right)}
\end{array}\right)
\end{aligned}
$$




\section{CHAPITRE 5}

\section{LE CAS GÉNÉRAL, RÉDUCTION AU CAS OÙ $\beta=\varnothing$}

\subsection{Le théorème de réduction}

Soient $n, h, k$ trois entiers positifs tels que $h+k=n$. Soit $\mathbf{G}=\mathbf{G L}(2 n+1)$. On le munit de l'involution $\theta$ définie par la formule 2.1. Soit $\beta=\left(\beta_{1}, \ldots, \beta_{t}\right)$ une partition de $h$ et soit $\pi_{0}$ une représentation lisse, irréductible $\theta$-stable de $G L(2 k+1)$. Avec les notations usuelles, définissons l'induite parabolique

$$
\pi=\mathrm{St}_{\beta_{1}} \times \cdots \times \mathrm{St}_{\beta_{t}} \times \pi_{0} \times \mathrm{St}_{\beta_{t}} \times \cdots \times \mathrm{St}_{1}
$$

C'est une représentations de $G$. On suppose en plus que $\pi$ est irréductible, elle est alors lisse, irréductible et $\theta$-stable.

Soit $\mathbf{P}=\mathbf{M N}$ le groupe parabolique standard de $\mathbf{G}$ correspondant à la partition $(h, 2 k+1, h)$. On a :

$$
M=\left\{m=\left(\begin{array}{ccc}
m_{1} & & 0 \\
& m_{0} & \\
0 & & m_{2}
\end{array}\right) ; m_{1}, m_{2} \in G L(h), m_{0} \in G L(2 k+1)\right\}
$$

Posons : $M_{1}=\left\{m \in M ; m_{0}=\mathrm{Id}, m_{2}=\mathrm{Id}\right\}$, et

$$
\begin{aligned}
\tau_{1}: G L(h) & \longrightarrow M_{1} \\
m_{1} & \longmapsto\left(\begin{array}{lll}
m_{1} & & 0 \\
& \text { Id } & \\
& & \text { Id }
\end{array}\right)
\end{aligned}
$$

Les groupes $M_{0}$ et $M_{2}$ ainsi que les isomorphismes $\tau_{0}, \tau_{2}$ sont définis de façon analogue. L'involution $\theta$ laisse stable $M$ ainsi que $M_{0}$, et via $\tau_{0}$ induit une involution sur $G L(2 k+1)$ que l'on va encore noter par $\theta$ si aucune confusion n'est à craindre. On déduit de $\theta$ également une involution sur $G L(h)$ par la formule : $h \in G L(h) \mapsto$ $\tau_{2}^{-1} \theta \tau_{1}(h) \in G L(h)$. C'est l'involution $\theta$ sur $G L(h)$. Avec ces définitions, les représentations $\pi$ et $\pi_{0}$ sont lisses, irréductibles, $\theta$-stables, de même que $\mathrm{St}_{\beta_{1}} \times \cdots \times \mathrm{St}_{\beta_{t}}$. 
On peut donc parler de traces tordues, tordues compactes de ces représentations sans ambiguïtés.

Pour tout $i$ entier positif, soit $p_{i}$ l'homomorphisme d'algèbres défini de la façon suivante :

$$
\begin{aligned}
p_{i}: \mathbb{C}\left[X_{1}^{ \pm}, \ldots, X_{2 i+1}^{ \pm}\right]^{\mathfrak{S}_{2 i+1}} \longrightarrow \mathbb{C}\left[X_{1}^{ \pm}, \ldots, X_{i}^{ \pm}\right]^{W\left(C_{i}\right)} \\
X_{j} \longmapsto p_{i}\left(X_{j}\right)= \begin{cases}X_{j} & \text { si } 1 \leqslant j \leqslant i \\
1 & \text { si } j=i+1 \\
X_{2 i+2-j}^{-1} & \text { si } i+2 \leqslant j \leqslant 2 i+1\end{cases}
\end{aligned}
$$

Posons :

$$
\widehat{T}_{u, i}=\left\{\left(x_{1}, \ldots, x_{i}\right) \in \mathbb{C}^{i} ;\left|x_{1}\right|=\cdots=\left|x_{i}\right|=1\right\}
$$

qui est muni de la mesure de Haar pour laquelle le volume de $\widehat{T}_{u, i}$ est égal à 1 . Pour simplifier, nous supprimerons les indices $i$ lorsque $i=n$.

Notons toujours $S: \mathcal{H}(G L(i)) \longrightarrow \mathbb{C}\left[X_{1}^{ \pm}, \ldots, X_{i}^{ \pm}\right]^{\mathfrak{S}_{i}}$ l'isomorphisme de Satake. Posons :

$$
Q_{i}\left(x_{1}, \ldots, x_{i}\right)=\prod_{j=1}^{i-1} \frac{1}{1-q x_{j} x_{j+1}^{-1}}
$$

Pour toute partition $\gamma=\left(\gamma_{1}, \ldots, \gamma_{t}\right)$ de somme $S(\gamma)$, on définit la fonction $Q_{\gamma}$ par la formule :

$$
Q_{\gamma}\left(x_{1}, \ldots, x_{S(\gamma)}\right)=Q_{\gamma_{1}}\left(x_{1}, \ldots, x_{\gamma_{1}}\right) \cdots Q_{\gamma_{t}}\left(x_{S(\gamma)-\gamma_{t}+1}, \ldots, x_{S(\gamma)}\right)
$$

Le but de ce chapitre est de démontrer le résultat suivant :

THÉORÈME 5.3. - Supposons qu'il existe une fonction rationnelle $Q_{\pi_{0}}$ en $k$ variables, n'ayant pas de pôle sur $\widehat{T}_{u, k}$ telle que pour toute fonction $f$ de l'algèbre de Hecke sphérique de $G L(2 k+1)$ on ait l'égalité :

$$
\operatorname{tr}_{\theta-c} \pi_{0}(f)=\int_{\widehat{T}_{u, k}} p_{k}(S(f))\left(x_{1}, \ldots, x_{k}\right) Q_{\pi_{0}}\left(x_{1}, \ldots, x_{k}\right) d^{\times} x_{1} \cdots d^{\times} x_{k}
$$

Alors, pour tout élément $f$ de l'algèbre de Hecke sphérique de $G L(2 n+1)$ on a l'identité :

$$
\operatorname{tr}_{\theta-c} \pi(f)=\int_{\widehat{T}_{u}} p(S(f))\left(x_{1}, \ldots, x_{n}\right) Q_{\beta}\left(x_{1}, \ldots, x_{h}\right) Q_{\pi_{0}}\left(x_{h+1}, \ldots, x_{n}\right) d^{\times} x_{1} \cdots d^{\times} x_{n}
$$

Rappelons que l'on s'intéressait aux représentations $\pi$ paramétrées par un ensemble $\mathcal{A}^{\text {st }}(n)$ de triplets de partitions $\left(\alpha^{+}, \alpha^{-}, \beta\right)$. Le théorème précédent réduit le problème de calcul de traces tordues compactes de telles $\pi$ à celui de celles des $\pi_{0}$ paramétrées par les triplets de la forme $\left(\alpha^{+}, \alpha^{-}, \beta=\varnothing\right)$. 


\subsection{Démonstration du théorème 5.3}

Avant toute chose, remarquons qu'il suffira de prouver le théorème précédent dans le cas où la partition $\beta$ est réduite à un seul terme, les autres cas s'en déduisant immédiatement par récurrence. Supposons désormais que $\beta=(h)$.

5.2.1. Opérateur d'entrelacement $A: \pi \simeq \pi^{\theta}$. - Soient $E, V_{0}$ les espaces de représentations de $\mathrm{St}_{h}$ et $\pi_{0}$ respectivement. L'espace $V$ de $\pi$ est alors défini par :

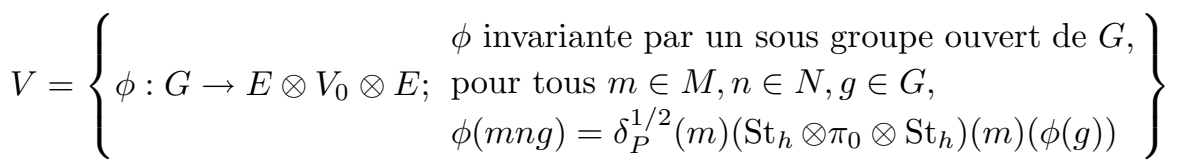

Fixons :

- $A_{0} \in \operatorname{End}_{\mathbb{C}}\left(V_{0}\right)$, un opérateur qui entrelace $\pi_{0}$ et $\pi_{0}^{\theta}$;

- $A_{S t} \in \operatorname{End}_{\mathbb{C}}(E)$, un opérateur qui entrelace $\mathrm{St}_{h}$ et $\mathrm{St}_{h}^{\theta}$.

On normalise $A_{S t}$ comme en 2.3. En particulier, on a : $A_{S t}^{2}=1$. Posons :

$$
\begin{aligned}
B: E \otimes V_{0} \otimes E & \longrightarrow E \otimes V_{0} \otimes E \\
e \otimes v \otimes e^{\prime} & \longmapsto A_{S t}\left(e^{\prime}\right) \otimes A_{0}(v) \otimes A_{S t}(e)
\end{aligned}
$$

Rappelons que pour toute fonction $\phi$ sur $G$ la fonction $\phi^{\theta}$ est définie par la formule $\phi^{\theta}(g)=\phi(\theta(g))$. On définit l'opérateur $A \in \operatorname{End}_{\mathbb{C}}(V)$ par la formule :

$$
\begin{aligned}
A: V & \longrightarrow V \\
\phi & \longmapsto A(\phi): g \longmapsto A(\phi)(g)=B\left(\phi^{\theta}(g)\right)
\end{aligned}
$$

L'opérateur $A$ est alors un opérateur qui entrelace $\pi$ et $\pi^{\theta}$. Nous l'utilisons pour calculer la trace tordue compacte de $\pi$.

5.2.2. Réduction aux espaces de dimension finie. - Notons $\mathbf{1}_{\theta-c}$ la fonction caractéristique de $G_{\theta-c}$, l'ensemble des éléments $\theta$-compacts de $G$. Pour toute fonction $f$ sur $G$, posons : $f_{\theta-c}=\mathbf{1}_{\theta-c} f$. La trace tordue compacte d'une fonction localement constante, à support compact $f$ est, par définition, la trace de l'opérateur :

$$
A \circ \pi\left(f_{\theta-c}\right)=\int_{G} A \circ \pi(g) f_{\theta-c}(g) d g
$$

Fixons un élément $f$ de $\mathcal{H}$, l'algèbre de Hecke sphérique de $G$. On a le lemme suivant :

LEMME 5.4. - Il existe un sous-groupe ouvert compact $H$ de $G$ avec les propriétés :

(1) $H$ est un sous groupe distingué de $K$, et $\theta$-invariant;

(2) Le support de $f_{\theta-c}$, resp. de $f_{\theta-c}^{P}$, est invariant par $H$, resp. par $H \cap M$;

(3) $H$ est en bonne position par rapport à $\mathbf{M}$, ce qui signifie que l'on a les décompositions :

$$
H=(H \cap \bar{N})(H \cap M)(H \cap N), H \cap M=\left(H \cap M_{1}\right)\left(H \cap M_{0}\right)\left(H \cap M_{2}\right)
$$

où, comme c'est usuel, $\overline{\mathbf{N}}$ est le radical unipotent du parabolique opposé à $\mathbf{P}$.

Démonstration. - Omise. 
Fixons pour le reste de cette section un groupe $H$ vérifiant les conditions énoncées dans le lemme précédent. On le munit également de la mesure de Haar pour laquelle le volume de $H$ est égal à 1 . Posons, pour $i=0,1,2: H_{i}=\tau_{i}^{-1}\left(H \cap M_{i}\right)$. On vérifie qu'alors $H_{1}=H_{2}$ et est un sous-groupe de $G L(h)$, et $H_{0}$ est un sous-groupe de $G L(2 k+1)$. Pour raisons de commodité, on va noter le groupe $H_{1}=H_{2}$ par $H_{12}$.

L'opérateur $A \circ \pi\left(f_{\theta-c}\right)$ est un opérateur à image dans $V^{H}$, les invariants par $H$. Pour calculer la trace de $A \circ \pi\left(f_{\theta-c}\right)$, il suffit donc de calculer celle de sa restriction à $V^{H}$, qui est un espace de dimension finie. Une base de cet espace est obtenue de la façon suivante. Fixons :

- un ensemble de représentants de $P \cap K \backslash K / H$ que l'on va noter $[P \cap K \backslash K / H]$;

- une base $\left\{e_{i}\right\}_{i \in I}$ de $E^{H_{12}}$;

- une base $\left\{v_{j}\right\}_{j \in J}$ de $V_{0}^{H_{0}}$;

- ainsi que la base $\left\{e_{k}^{\prime}\right\}_{k \in I}$ de $E^{H_{12}}$ définie par $e_{k}^{\prime}=A_{S t}\left(e_{k}\right)$.

Pour $(x, i, j, k) \in[P \cap K \backslash K / H] \times I \times J \times I$, soit $\phi_{x, i, j, k}$ l'unique élément de $V$ ayant les propriétés suivantes :

(1) $\phi_{x, i, j, k}$ est à support dans $P x H$;

(2) $\phi_{x, i, j, k}(x)=e_{i} \otimes v_{j} \otimes e_{j}^{\prime}$.

Lorsque $(x, i, j, k)$ parcourt $[P \cap K \backslash K / H] \times I \times J \times I$, les $\phi_{x, i, j, k}$ forment une base de $V^{H}$.

5.2.3. Action de $A \circ \pi\left(f_{\theta-c}\right)$ sur la base $\left\{\phi_{x, i, j, k}\right\}$ - - Fixons un élément $\phi_{x, i, j, k}$ et pour simplifier, nous écrivons $\phi$ à la place de $\phi_{x, i, j, k}$. Soit $g_{0} \in G$, il vient alors des définitions que :

$$
A \circ \pi\left(f_{\theta-c}\right)(\phi)\left(g_{0}\right)=B\left(\int_{G} \phi(g) f_{\theta-c}\left(\theta\left(g_{0}^{-1}\right) g\right) d g\right)
$$

Nous allons décomposer l'intégration dans la formule 5.5 en une intégration sur $P H$. Fixons un système de représentants $[K / H]$ de $K / H$. Pour $x \in[P \cap K \backslash K / H]$, posons :

$$
\mu(x)=\frac{\operatorname{card}\{y \in[K / H] ; y \in(P \cap K) x H\}}{\operatorname{card}[K / H]}
$$

On a alors l'égalité élémentaire suivante :

$$
\sum_{x \in[P \cap K \backslash K / H]} \mu(x)=1
$$

Lemme 5.7. - Pour toute fonction $\varphi$ intégrable sur $G$, on a l'égalité :

$$
\int_{G} \varphi(g) d g=\sum_{x \in[P \cap K \backslash K / H]} \mu(x) \int_{P H} \varphi(p x h) d h d p
$$

Démonstration. — En effet, cela résulte de la décomposition d'Iwasawa :

$$
\int_{G} \varphi(g) d g=\int_{P K} \varphi(p k) d k d p
$$


et une décomposition élémentaire de l'intégration de $K$ en une intégration sur $K \backslash H \times H$.

D'après les formules $5.5,5.6$, et 5.8 ,

$$
\begin{aligned}
A \circ \pi\left(f_{\theta-c}\right)(\phi)\left(g_{0}\right) & =B\left(\sum_{y \in[P \cap K \backslash K / H]} \mu(y) \int_{P H} \phi(p y h) f_{\theta-c}\left(\theta\left(g_{0}^{-1}\right) p y h\right) d h d p\right) \\
& =B\left(\sum_{y \in[P \cap K \backslash K / H]} \mu(y) \int_{P} \phi(p y) f_{\theta-c}\left(\theta\left(g_{0}^{-1}\right) p y\right) d p\right) \\
& =B\left(\mu(x) \int_{P} \phi(p x) f_{\theta-c}\left(\theta\left(g_{0}^{-1}\right) p x\right) d p\right) \\
& =\mu(x) B\left(\int_{M N}\left(\mathrm{St}_{h} \otimes \pi_{0} \otimes \mathrm{St}_{h}\right)(m)\left(e_{i} \otimes v_{j} \otimes e_{k}^{\prime}\right) \times\right. \\
& \left.\times \delta_{P}^{1 / 2}(m) f_{\theta-c}\left(\theta\left(g_{0}^{-1}\right) m n x\right) d n d m\right)
\end{aligned}
$$

La deuxième égalité vient de la $H$-invariance des fonctions $\phi$ et $f_{\theta-c}$, la troisième vient du fait que le support de $\phi$ est contenu dans $P x H$.

5.2.4. Décomposition de $\operatorname{tr}_{\theta-c} \pi(f)$. — Pour calculer la trace de $A \circ \pi\left(f_{\theta-c}\right)$, il suffit de calculer la valeur de $A \circ \pi\left(f_{\theta-c}\right)(\phi)$ en $g_{0}=x$. On a :

$$
\begin{aligned}
f_{\theta-c}\left(\theta\left(x^{-1}\right) m n x\right) & =f\left(\theta\left(x^{-1}\right) m n x\right) \mathbf{1}_{\theta-c}\left(\theta\left(x^{-1}\right) m n x\right) \\
& =f(m n) \mathbf{1}_{\theta-c}(m n)
\end{aligned}
$$

car la fonction $f$ est biinvariante par $K$, et la fonction $\mathbf{1}_{\theta-c}$ est invariante par $\theta$ conjugaison.

Lemme 5.11. - Pour tous $m \in M, n \in N$, on a l'égalité :

$$
\mathbf{1}_{\theta-c}(m n)=\mathbf{1}_{\theta-c}(m)
$$

Admettons provisoirement ce lemme. On déduit des égalités 5.9,5.10, et 5.12 :

$$
\begin{aligned}
A \circ \pi\left(f_{\theta-c}\right)(\phi)(x)=\delta(x) B\left(\int_{M N}\left(\mathrm{St}_{h} \otimes \pi_{0} \otimes \mathrm{St}_{h}\right)(m)\left(e_{i} \otimes v_{j} \otimes e_{k}^{\prime}\right) \times\right. \\
\left.\times \delta_{P}^{1 / 2}(m) f(m n) \mathbf{1}_{\theta-c}(m) d n d m\right) \\
=\delta(x) B\left(\int_{M}\left(\mathrm{St}_{h} \otimes \pi_{0} \otimes \mathrm{St}_{h}\right)(m)\left(e_{i} \otimes v_{j} \otimes e_{k}^{\prime}\right)\left(f^{P}\right)_{\theta-c}(m) d m\right)
\end{aligned}
$$

où $f^{P}$ est le transfert de $f$ le long du parabolique $P$.

Soient $\left\{\check{e}_{i}\right\}_{i \in I},\left\{\check{v}_{j}\right\}_{i \in J},\left\{\check{e}_{k}^{\prime}\right\}_{k \in I}$ les bases duales des bases $\left\{e_{i}\right\}_{i \in I},\left\{v_{j}\right\}_{i \in J},\left\{e_{k}^{\prime}\right\}_{k \in I}$, respectivement. On a :

$$
\operatorname{tr}_{\theta-c} \pi(f)=\int_{M} \Theta(m)\left(f^{P}\right)_{\theta-c}(m) d m
$$


où

$$
\Theta(m)=\sum_{x \in[P \cap K \backslash K / H]} \delta(x) \sum_{i, j, k}\left\langle\check{e}_{i} \otimes \check{v}_{j} \otimes \check{e}_{k}, B\left(\mathrm{St}_{h} \otimes \pi_{0} \otimes \mathrm{St}_{h}\right)(m)\left(e_{j} \otimes v_{j} \otimes e_{k}\right)\right\rangle
$$

La somme des $\mu(x)$ vaut 1 , d'après la formule 5.6. En écrivant $m=\left(m_{1}, m_{0}, m_{2}\right)$, on peut donc décomposer :

$$
\Theta\left(m_{1}, m_{0}, m_{2}\right)=\Theta_{12}\left(m_{1}, m_{2}\right) \Theta_{0}\left(m_{0}\right)
$$

où

$$
\begin{aligned}
\Theta_{0}\left(m_{0}\right) & =\sum_{j}\left\langle\check{v}_{j}, A_{0} \circ \pi_{0}\left(m_{0}\right) v_{j}\right\rangle \\
\Theta_{12}\left(m_{1}, m_{2}\right) & \left.=\sum_{i, k}\left\langle\check{e}_{i} \otimes \check{e}_{k}^{\prime}, A_{\mathrm{st}} \circ \operatorname{St}_{h}\left(m_{2}\right) e_{k}^{\prime} \otimes A_{\mathrm{st}} \circ \mathrm{St}_{h}\left(m_{1}\right) e_{i}\right)\right\rangle
\end{aligned}
$$

On a :

$$
\begin{aligned}
\Theta_{0}\left(m_{0}\right) & =\operatorname{mes}\left(H_{0}\right)^{-1} \operatorname{tr}_{\theta-c} \pi_{0}\left(\mathbf{1}_{m_{0} H_{0}}\right) \\
\Theta_{12}\left(m_{1}, m_{2}\right) & \left.=\operatorname{mes}\left(H_{12}\right)^{-2} \sum_{i, k}\left\langle\check{e}_{i} \otimes \check{e}_{k}^{\prime}, A_{\mathrm{st}} \circ \operatorname{St}_{h}\left(\mathbf{1}_{m_{2} H_{12}}\right) e_{k}^{\prime} \otimes A_{\mathrm{st}} \circ \operatorname{St}_{h}\left(\mathbf{1}_{m_{1} H_{12}}\right) e_{i}\right)\right\rangle \\
& =\operatorname{mes}\left(H_{12}\right)^{-2} \sum_{k}\left\langle\check{e}_{k}^{\prime}, A_{\mathrm{st}} \circ \operatorname{St}_{h}\left(\mathbf{1}_{m_{1} H_{12}}\right) \circ A_{\mathrm{st}} \circ \operatorname{St}_{h}\left(\mathbf{1}_{m_{2} H_{12}}\right)\left(e_{k}^{\prime}\right)\right\rangle \\
& =\operatorname{mes}\left(H_{12}\right)^{-2} \operatorname{tr} \operatorname{St}_{h}\left(\mathbf{1}_{m_{1} H_{12}} * \mathbf{1}_{\theta\left(m_{2}\right) H_{12}}\right)
\end{aligned}
$$

Pour une fonction $\varphi$ à support compact sur $M$, définissons $\widetilde{\varphi}$ sur $G L(h) \times G L(2 k+1)$ par :

$$
\widetilde{\varphi}\left(\widetilde{m}, m_{0}\right)=\int_{G L(h)} \varphi\left(\widetilde{m} m_{2}^{-1}, m_{0}, \theta\left(m_{2}\right)\right) d m_{2}
$$

Lemme 5.14. - Pour toute telle $\varphi$, invariante à droite par $H \cap M$, on a :

$$
\int_{M} \Theta(m) \varphi(m) d m=\left(\operatorname{tr} S t \otimes \operatorname{tr}_{\theta} \pi_{0}\right)(\widetilde{\varphi})
$$

Démonstration. - En effet, il suffit de le vérifier pour $\varphi=\mathbf{1}_{m_{1} H_{12}} * \mathbf{1}_{m_{0} H_{0}} * \mathbf{1}_{m_{2} H_{12}}$, et cela résulte de ci-dessus.

En appliquant successivement les formules $5.13,5.15$, on obtient :

$$
\operatorname{tr}_{\theta-c} \pi(f)=\left(\operatorname{tr} S t \otimes \operatorname{tr}_{\theta} \pi_{0}\right)\left(\widetilde{\left(f^{P}\right)_{\theta-c}}\right)
$$

Notons $G L(h)_{c}$ l'ensemble des éléments compacts de $G L(h)$. C'est l'ensemble des éléments de $G$ dont les valeurs propres dans l'algèbre de Lie de $G L(h)$ sont toutes de module 1. Notons $G L(h)_{0}$ l'ensemble des éléments de $G L(h)$ déterminant de module 1. On note $\mathbf{1}_{\mathbf{c}}, \mathbf{1}_{\mathbf{0}}$ les fonctions caractéristiques de $G L(h)_{c}$ et $G L(h)_{0}$ respectivement. On a deux lemmes suivants : 
Lemme 5.16. - L'ensemble $M \cap G_{\theta-c}$ est, à un ensemble de mesure nulle près, l'ensemble des éléments $m=m_{1} m_{0} m_{2} \in M$ vérifiant les conditions :

- $m_{0}$ est un élément de $G L(2 k+1)_{\theta-c}$;

- $m_{1} \theta\left(m_{2}\right)$ est un élément de $G L(h)_{c} \cap G L(h)_{0}$.

Ce lemme sera démontré en 5.2.8. Le deuxième est trivial :

Lemme 5.17. - Il existe un ensemble fini $\mathcal{L}$ et des fonctions sphériques $\left\{f_{l, 1}, f_{l, 0}, f_{l, 2}\right\}_{l \in \mathcal{L}}$ sur $M_{1}, M_{0}, M_{2}$ respectivement telles que, pour tout $m=m_{1} m_{0} m_{2}$ on ait l'égalité suivante :

$$
f^{P}(m)=\sum_{l \in \mathcal{L}} f_{l, 1}\left(m_{1}\right) f_{l, 0}\left(m_{0}\right) f_{l, 2}\left(m_{2}\right)
$$

D'après le lemme 5.16,

$$
\left(\widetilde{f^{P} \mathbf{1}_{\theta-c}}\right)\left(\widetilde{m}, m_{0}\right)=\widetilde{f^{P}}\left(\widetilde{m}, m_{0}\right) \mathbf{1}_{0}(\widetilde{m}) \mathbf{1}_{c}(\widetilde{m}) \mathbf{1}_{c}\left(m_{0}\right)
$$

D'après le lemme 5.17 , on a :

$$
\widetilde{f^{P}}\left(\widetilde{m}, m_{0}\right)=\sum_{l \in \mathcal{L}} f_{l, 1} * f_{l, 2}^{\theta}(\widetilde{m}) f_{l, 0}\left(m_{0}\right)
$$

On en déduit :

Proposition 5.18. - On a l'égalité :

$$
\operatorname{tr}_{\theta-c} \pi(f)=\sum_{l \in \mathcal{L}} \operatorname{tr}_{c} \operatorname{St}_{h}\left(\mathbf{1}_{0}\left(f_{l, 1} * f_{l, 2}^{\theta}\right)\right) \operatorname{tr}_{\theta-c} \pi_{0}\left(f_{l, 0}\right)
$$

5.2.5. Le terme $\operatorname{tr}_{c} \mathrm{St}_{h}$. - Pour calculer la trace compacte de $\mathrm{St}_{h}$, on dispose de la formule de Clozel (formule du Th.1.7 pour $\theta$ trivial). Rappelons-la brièvement en reprenant les notations du premier chapitre. Soit $\mathcal{P}$ l'ensemble des groupes paraboliques standards de $\mathbf{G L}(h)$. Pour $\mathbf{P}=\mathbf{M N} \in \mathcal{P}$, soit $\widehat{\chi}_{N}$ la composée de la fonction de Harish-Chandra avec la fonction caractéristique de la chambre de Weyl obtuse positive dans l'algèbre de Lie réelle du centre de $M$ associée à l'ensemble des racines simples dans $N$. Notons $a_{P}$ la dimension de cette algèbre de Lie.

Soit $\pi$ une représentation lisse, admissible de $G L(h)$. Pour tout $f \in \mathcal{C}_{c}^{\infty}(G L(h))$, on a alors l'égalité :

$$
\operatorname{tr}_{c} \pi(f)=\sum_{\mathbf{P}=\mathbf{M N} \in \mathcal{P}}(-1)^{a_{P}-a_{G L(h)}} \operatorname{tr} \pi_{N}\left(\widehat{\chi}_{N} f^{P}\right)
$$

Appliquons cette formule à $\pi=\operatorname{St}_{h}$ et $f=\mathbf{1}_{0}\left(f_{l, 1} * f_{l, 2}^{\theta}\right)$. La fonction $\mathbf{1}_{0}\left(f_{l, 1} * f_{l, 2}^{\theta}\right)$ est une fonction sphérique sur $G L(h)$ car $f_{l, 1}, f_{l, 2}^{\theta}$ le sont, et la fonction $\mathbf{1}_{0}$ est biinvariante par $G L\left(h, O_{F}\right)$. D'autre part, pour $\mathbf{P}=\mathbf{M N} \in \mathcal{P}$, le module de Jacquet $\pi_{N}$ n'a pas de vecteurs sphériques non nuls sauf si $\mathbf{P}=\mathbf{B}$ le Borel triangulaire supérieur, auquel 
cas, le module de Jacquet correspondant est le caractère non ramifié suivant du tore diagonal :

On en déduit :

$$
\eta=\left(\frac{h-1}{2}, \frac{h-3}{2}, \ldots, \frac{1-h}{2}\right)
$$

$$
\operatorname{tr}_{c} \operatorname{St}_{h}\left(\mathbf{1}_{0}\left(f_{l, 1} * f_{l, 2}^{\theta}\right)\right)=(-1)^{a_{B}-a_{G L(h)}} \operatorname{tr} \eta\left(\widehat{\chi}_{U}\left(\mathbf{1}_{0}\left(f_{l, 1} * f_{l, 2}^{\theta}\right)^{B}\right)\right.
$$

On a :

$$
a_{B}-a_{G L(h)}=h-1
$$

Quant au terme $\widehat{\chi}_{U} \mathbf{1}_{0}\left(f_{l, 1} * f_{l, 2}^{\theta}\right)^{B}$, on a le résultat suivant. Écrivons les transformées de Satake des fonctions $f_{l, 1}, f_{l, 2}$ :

$$
\begin{aligned}
S\left(f_{l, 1}\right)\left(X_{1}, \ldots, X_{h}\right) & =\sum_{\boldsymbol{m}=\left(m_{1}, \ldots, m_{h}\right) \in \mathbb{Z}^{h}} a_{l, \boldsymbol{m}} X_{1}^{m_{1}} \cdots X_{h}^{m_{h}} \\
S\left(f_{l, 2}\right)\left(X_{2 n+2-h}, \ldots, X_{2 n+1}\right)= & \sum_{\mathbf{m}^{\prime}=\left(m_{2 n+1-h}, \ldots, m_{2 n+1}\right) \in \mathbb{Z}^{h}} a_{l, \mathbf{m}^{\prime}} X_{2 n+2-h}^{m_{2 n+2}} \cdots X_{2 n+1}^{m_{2 n+1}}
\end{aligned}
$$

On a :

$$
S\left(f_{l, 2}^{\theta}\right)\left(X_{1}, \ldots, X_{h}\right)=\sum_{\mathbf{m}^{\prime}=\left(m_{2 n+1-h}, \ldots, m_{2 n+1}\right) \in \mathbb{Z}^{h}} a_{l, \mathbf{m}^{\prime}} X_{1}^{-m_{2 n+1}} \cdots X_{h}^{-m_{2 n+2-h}}
$$

On en déduit :

$$
S\left(f_{l, 1} * f_{l, 2}^{\theta}\right)\left(X_{1}, \ldots, X_{h}\right)=\sum_{\left(\mathbf{m}, \mathbf{m}^{\prime}\right) \in \mathbb{Z}^{h} \times \mathbb{Z}^{h}} a_{l, \boldsymbol{m}} a_{l, \mathbf{m}^{\prime}} X_{1}^{m_{1}-m_{2 n+1}} \cdots X_{h}^{m_{h}-m_{2 n+2-h}}
$$

Posons :

$$
\mathcal{M}=\left\{\left(\mathbf{m}, \mathbf{m}^{\prime}\right) \in \mathbb{Z}^{h} \times \mathbb{Z}^{h} ; \begin{array}{l}
\text { pour tout } i=1, \cdots, h-1: \\
m_{1}+\cdots+m_{i}-m_{2 n+2-i}-\cdots-{ }_{2 n+1}>0 \\
m_{1}+\cdots m_{h}-m_{2 n+2-h}-\cdots-m_{2 n+1}=0
\end{array}\right\}
$$

Le résultat suivant résulte alors des calculs élémentaires :

Lemme 5.20. - La transformée de Satake de la fonction $\widehat{\chi}_{U} \mathbf{1}_{0}\left(f_{l, 1} * f_{l, 2}^{\theta}\right)^{B}$ est donnée par la formule suivante:

$S\left(\widehat{\chi}_{U} \mathbf{1}_{0}\left(f_{l, 1} * f_{l, 2}^{\theta}\right)^{B}\right)\left(X_{1}, \ldots, X_{h}\right)=\sum_{\left(\mathbf{m}, \mathbf{m}^{\prime}\right) \in \mathcal{M}} a_{l, \boldsymbol{m}} a_{l, \mathbf{m}^{\prime}} X_{1}^{m_{1}-m_{2 n+1}} \cdots X_{h}^{m_{h}-m_{2 n+2-h}}$

L'égalité suivante se déduit alors de ce qui précède :

$$
\begin{aligned}
\operatorname{tr}_{c} \operatorname{St}_{h}\left(\mathbf{1}_{0}\left(f_{l, 1} * f_{l, 2}^{\theta}\right)\right) & \\
& =(-1)^{h-1} \sum_{\left(\mathbf{m}, \mathbf{m}^{\prime}\right) \in \mathcal{M}} a_{l, \boldsymbol{m}} a_{l, \mathbf{m}^{\prime}} q^{\frac{1-h}{2}\left(m_{1}-m_{2 n+1}\right)+\cdots+\frac{h-1}{2}\left(m_{h}-m_{2 n+2-h}\right)}
\end{aligned}
$$


5.2.6. Transformation de Satake du terme $\operatorname{tr}_{c} \mathrm{St}_{h}$. - Posons :

$$
\begin{gathered}
\mathcal{N}=\left\{\boldsymbol{n}=\left(n_{1}, \ldots, n_{h}\right) \in \mathbb{Z}^{h} ; \begin{array}{l}
\text { pour tout } i=1, \ldots h-1 \\
n_{1}+n_{2}+\cdots+n_{i}>0 \\
n_{1}+n_{2}+\cdots+n_{h}=0
\end{array}\right\} \\
P\left(x_{1}, \ldots, x_{h}\right)=(-1)^{h-1} \sum_{n \in \mathcal{N}} q^{\frac{1-h}{2} n_{1}+\frac{3-h}{2} n_{2} \cdots+\frac{h-1}{2} n_{h}} x_{1}^{-n_{1}} x_{2}^{-n_{2}} \cdots x_{h}^{-n_{h}}
\end{gathered}
$$

Pour alléger les notations, nous allons écrire simplement $S_{l}$ pour $S\left(\widehat{\chi}_{U} \mathbf{1}_{0}\left(f_{l, 1} * f_{l, 2}^{\theta}\right)^{B}\right)$. Par application de la formule d'orthogonalité 3.20, on déduit des égalités 5.21, 5.22:

$$
\operatorname{tr}_{c} \operatorname{St}_{h}\left(\mathbf{1}_{0}\left(f_{l, 1} * f_{l, 2}^{\theta}\right)\right)=\int_{\widehat{T}_{u, h}} S_{l}\left(x_{1}, \ldots, x_{h}\right) P\left(x_{1}, \ldots, x_{h}\right) d^{\times} x_{1} d^{\times} x_{2} \cdots d^{\times} x_{h}
$$

pourvu que la somme $P\left(x_{1}, \ldots, x_{h}\right)$ converge. En effet, on vérifie par un calcul élémentaire que cette somme converge absolument et uniformément sur $\widehat{T}_{u, h}$ vers la fonction $Q_{h}\left(x_{1}, \ldots, x_{h}\right)$ définie par la formule 5.2. On en déduit :

Proposition 5.23. - On a l'identité :

$$
\operatorname{tr}_{c} \operatorname{St}_{h}\left(\mathbf{1}_{0}\left(f_{l, 1} * f_{l, 2}^{\theta}\right)\right)=\int_{\widehat{T}_{u, h}} S_{l}\left(x_{1}, \ldots, x_{h}\right) Q_{h}\left(x_{1}, \ldots, x_{h}\right) d^{\times} x_{1} d^{\times} x_{2} \cdots d^{\times} x_{h}
$$

5.2.7. Fin de la démonstration. — Par hypothèse, on a l'égalité :

$$
\operatorname{tr}_{\theta-c} \pi_{0}\left(f_{l, 0}\right)=\int_{\widehat{T}_{u, k}} p_{k}\left(S\left(f_{l, 0}\right)\right)\left(x_{1}, \ldots, x_{k}\right) Q_{\pi_{0}}\left(x_{1}, \ldots, x_{k}\right) d^{\times} x_{1} \cdots d^{\times} x_{k}
$$

Posons :

$$
Q_{\pi}\left(x_{1}, \ldots, x_{n}\right)=Q_{h}\left(x_{1}, \ldots, x_{h}\right) Q_{\pi_{0}}\left(x_{h+1}, \ldots, x_{n}\right)
$$

D'après ce qui précède, le produit $\operatorname{tr}_{c} \operatorname{St}_{h}\left(\mathbf{1}_{0}\left(f_{l, 1} * f_{l, 2}^{\theta}\right)\right) \operatorname{tr}_{\theta-c} \pi_{0}\left(f_{l, 0}\right)$ est égal à

$$
\int_{\widehat{T}_{u}} S_{l}\left(x_{1}, \ldots, x_{h}\right) p_{k}\left(S\left(f_{l, 0}\right)\right)\left(x_{h+1}, \ldots, x_{n}\right) Q_{\pi}\left(x_{1}, \ldots, x_{n}\right) d^{\times} x_{h+1} \cdots d^{\times} x_{n}
$$

On sait, d'après la proposition 5.18 , que :

$$
\operatorname{tr}_{c} \pi(f)=\sum_{l \in \mathcal{L}} \operatorname{tr}_{c} \operatorname{St}_{h}\left(\mathbf{1}_{0}\left(f_{l, 1} * f_{l, 2}^{\theta}\right)\right) \operatorname{tr}_{\theta-c} \pi_{0}\left(f_{l, 0}\right)
$$

Par ailleurs, il vient des définitions que :

$$
p(f)\left(x_{1}, \ldots, x_{n}\right)=\sum_{l \in \mathcal{L}} S_{l}\left(x_{1}, \ldots, x_{h}\right) p_{k}\left(S\left(f_{l, 0}\right)\right)\left(x_{h+1}, \ldots, x_{n}\right)
$$

La démonstration s'achève. 


\subsubsection{Démonstrations des lemmes 5.11 et 5.16}

Démonstration du lemme 5.11. — Posons :

$$
X=\operatorname{diag}(\underbrace{1, \ldots, 1}_{h}, \underbrace{0, \ldots, 0}_{2 k+1}, \underbrace{-1, \ldots,-1}_{h})
$$

Pour $i \in \mathbb{Z}$, on note $\mathfrak{G}_{i}$ l'espace propre correspondant à la valeur propre $i$ de l'opérateur $\operatorname{Ad}(X)$ dans $\mathfrak{G}$ l'algèbre de Lie de $G$. Les opérateurs $\theta$ et $\operatorname{Ad}(m)$ conservent chaque $\mathfrak{G}_{i}$, cela pour tout $m \in M$, où $\theta$ désigne la différentielle de l'involution $\theta$. On vérifie facilement que pour tout $n \in N$, l'opérateur $\operatorname{Ad}(n)$ est tel que :

$$
\operatorname{Ad}(n)-1: \mathfrak{G}_{i} \longrightarrow \mathfrak{G}_{i+1}+\mathfrak{G}_{i+2}
$$

On en déduit que les polynômes caractéristiques des opérateurs $\theta \circ \operatorname{Ad}(m n)$ et $\theta \circ \operatorname{Ad}(m)$ sont égaux, et le lemme s'ensuit.

Démonstration du lemme 5.16. — Commençons par établir le lemme élémentaire suivant :

LEMme 5.24. - Soit $g$ un élément de G. Il y a équivalence entre :

- $g$ est $\theta$-compact;

- Les valeurs propres de $g \theta(g)$ sont toutes de module 1.

Démonstration. - En effet, dire que $g$ est $\theta$-compact, c'est dire que les valeurs propres de $\operatorname{Ad}(g) \circ \theta$ dans $\mathfrak{G}$ sont toutes de module 1 . Ceci est équivalent à dire que les valeurs propres de $\operatorname{Ad}(g \theta(g))=(\operatorname{Ad}(g) \circ \theta)^{2}$ dans cette algèbre de Lie sont, elles aussi, toutes de module 1. Ceci revient à dire que les valeurs propres de $\operatorname{Ad}(g \theta(g)$ sont toutes de même module. Or, on remarque que pour tout $g$, $\operatorname{det}(g \theta(g))=1$, ce qui force le module des valeurs propres de $\operatorname{Ad}(g \theta(g))$ à être égal à 1 .

Soit $m=\left(m_{1}, m_{0}, m_{2}\right) \in M$. On a alors $m \theta(m)=\left(m_{1} \theta\left(m_{2}\right), m_{0} \theta\left(m_{0}\right), m_{2} \theta\left(m_{1}\right)\right)$. Par application du lemme précédent, pour montrer le lemme 5.16, il suffira de montrer que $m_{1} \theta\left(m_{2}\right)$ est compact si et seulement si $m_{2} \theta\left(m_{1}\right)$ l'est. Or, $m_{1} \theta\left(m_{2}\right)$ est conjugué à $\theta\left(m_{2}\right) m_{1}$. Le reste résultera du fait suivant :

Lemme 5.25. - L'ensemble des éléments compacts de $G L(h)$ est stable par $\theta$.

Démonstration. - C'est élémentaire : il suffit de considérer un élément $g \in G L(h)$ triangulaire supérieur. Pour un tel élément, soient $\left(t_{1}, \ldots, t_{h}\right)$ les coefficients diagonaux. On vérifie alors que $\theta(g)$ est triangulaire supérieur dont les coefficients diagonaux sont, aux signes près, $\left(t_{h}^{-1}, \ldots, t_{1}^{-1}\right)$. Dire que $g$ est compact, c'est dire que les modules des $t_{i}$ sont égaux, ce qui est équivalent à dire que $\theta(g)$ est compact. 


\title{
CHAPITRE 6
}

\author{
LE CAS $\pi=\mathrm{St}_{2 n+1}$
}

Il s'agit du cas où $\left(\alpha^{+}, \alpha^{-}, \beta\right)=((2 n+1), \varnothing, \varnothing)$. Les résultats ci-dessous sont faciles, leurs démonstrations sont analogues à celles du cas $n=2$.

Proposition 6.1. - Soit $f \in \mathcal{H}$, et écrivons

$$
S(f)\left(X_{1}, \ldots, X_{2 n+1}\right)=\sum_{\boldsymbol{m}=\left(m_{1}, \ldots, m_{2 n+1}\right) \in \mathbb{Z}^{2 n+1}} c_{\boldsymbol{m}} X_{1}^{m_{1}} \cdots X_{2 n+1}^{m_{2 n+1}}
$$

Posons

$$
S_{1}=\left\{\boldsymbol{m}=\left(m_{1}, \ldots, m_{2 n+1}\right) \in \mathbb{Z}^{2 n+1} ; \text { pour tout } j, \quad \sum_{i=1}^{j} m_{i}-m_{2 n+2-i}>0\right\}
$$

On a l'égalité :

$$
\operatorname{tr}_{\theta-c} \pi(f)=\sum_{\boldsymbol{m} \in S_{1}} c_{\boldsymbol{m}} q^{\sum_{i=1}^{n}(n+1-i)\left(-m_{i}+m_{2 n+1-i}\right)}
$$

L'application $p$ a été définie au chapitre précédent ( $c f$. formule 5.1). On déduit de la proposition précédente le résultat suivant :

THÉORÈme 6.2. - Avec les mêmes notations que dans la proposition précédente, on a l'égalité :

$$
\begin{aligned}
& \operatorname{tr}_{\theta-c} \pi(f) \\
& \quad=\int_{\widehat{T}_{u}} \frac{1}{1-q x_{1} x_{2}^{-1}} \cdots \frac{1}{1-q x_{n-1} x_{n}^{-1}} \frac{1}{1-q x_{n}} p(S(f))\left(x_{1}, \ldots, x_{n}\right) d^{\times} x_{1} \cdots d^{\times} x_{n}
\end{aligned}
$$





\title{
CHAPITRE 7
}

\author{
LE CAS $\pi=\mathrm{St}_{2 h+1} \times \xi \mathrm{St}_{2 k+1} \times \xi \mathrm{St}_{1}$
}

Il s'agit du cas où $\left(\alpha^{+}, \alpha^{-}, \beta\right)=((2 h+1),(2 k+1,1), \varnothing)$, où $h, k$ sont deux entiers, $k \geqslant 1$, tels que $h+k+1=n$.

Le sous-groupe parabolique standard $\mathbf{R}=\mathbf{L V}$ est, dans le cas présent, paramétré par la partition $(2 h+1,2 k+1,1)$. Notons $V$ l'espace de $\pi$ et soit $A \in \operatorname{End}_{\mathbb{C}}(V)$ l'opérateur d'entrelacement entre $\pi$ et $\pi^{\theta}$ normalisé comme en 2.3. En vertu de la formule 1.7, le calcul de la trace tordue compacte $\operatorname{tr}_{\theta-c} \pi$ se ramène aux calculs des traces tordues des modules de Jacquet $\pi_{N}$ de $\pi$ où $\mathbf{P}=\mathbf{M N}$ est un sous-groupe parabolique standard $\theta$-stable.

\subsection{Le module de Jacquet $\pi_{U}$, où $\mathbf{B}=$ TU}

\subsubsection{Décomposition de $\pi_{U}$ en somme de sous-espaces caractéristiques}

On a posé $: \varepsilon=i \pi / \log q$. Le caractère non ramifié $\eta$ du tore diagonal $T$ ( $c f .2 .1 .3$ ) est donné par :

$$
\eta=(h, h-1, \ldots,-h, k+\varepsilon, k-1+\varepsilon, \ldots,-k+\varepsilon, \varepsilon)
$$

On note $W^{L}$ le groupe de Weyl de $L$, et $\left[W / W^{L}\right]$ le système des représentants distingués des classes à droite de $W^{L}$. Avec les notations usuelles, on a une décomposition de $V_{U}$, un $T$-module, en somme directe de sous-espaces caractéristiques :

$$
V_{U}=\oplus_{\chi} V_{U}[\chi]
$$

où la somme porte sur les caractères $\chi$ de la forme $w \eta, w \in\left[W / W^{L}\right]$. Notons encore par $A$ l'action déduite de $A$ sur $V_{U}$. Pour tout caractère complexe $\chi$ de $T$, on a : $A\left(V_{U}[\chi]\right)=V_{U}[\theta(\chi)]$. Considérons donc les caractères $\chi$ pour lesquels :

- $\quad \chi=w \eta$ pour un $w \in\left[W / W^{L}\right]$;

- $\quad \chi$ est $\theta$-invariant, c'est-à-dire $\theta(\chi)=\chi$.

Notons $\mathcal{C}(\pi)$ l'ensemble de tels caractères. Pour $\chi \in \mathcal{C}(\pi)$, et $f$ une fonction (continue, à support compact) sur $T$, l'opérateur $A \circ \pi_{U}(f)$ agit sur $V_{U}[\chi]$. Nous définissons 
$\operatorname{tr}_{\theta} \pi_{U}[\chi](f)$ comme la trace de cet opérateur. L'égalité suivante résulte alors des définitions :

$$
\operatorname{tr}_{\theta} \pi_{U}(f)=\sum_{\chi \in \mathcal{C}(\pi)} \operatorname{tr}_{\theta} \pi_{U}[\chi](f)
$$

Fixons un $\chi=\left(\chi_{1}, \chi_{2}, \ldots, \chi_{2 n+1}\right) \in \mathcal{C}(\pi)$ et calculons $\operatorname{tr}_{\theta} \pi_{U}[\chi](f)$.

7.1.2. Définition de $i_{\max }$. - On pose : $I(2 n+1)=[1,2 n+1]$, le segment des entiers compris entre 1 et $2 n+1$. Définissons les segments $I(h)$ et $I(k)$ par :

$$
\begin{aligned}
& I(h)=[1,2 h+1] \\
& I(k)=[2 h+2,2 h+2 k+2=2 n]
\end{aligned}
$$

Posons : $a_{i}=h-i+1, b_{j}=2 h+2+k-j$, de sorte que $I(h)=\left[a_{h}, a_{-h}\right], I(k)=\left[b_{k}, b_{-k}\right]$. On a donc $\eta_{a_{i}}=i, \eta_{b_{j}}=j+\varepsilon$. Dire que $w \in\left[W / W^{L}\right]$, c'est dire que $w$ est croissant sur les segments $I(h)$ et $I(k)$.

Puisque $\chi$ est de la forme $w \eta$, il y a exactement deux termes $\varepsilon$ parmi les $\chi_{i}$. Par symétrie, si $\chi_{i}=\varepsilon$, alors $\chi_{2 n+2-i}=\varepsilon$. On peut donc définir l'entier $i_{\max }$ comme l'unique entier positif tel que :

$$
\chi_{n+1-i_{\max }}=\chi_{n+1+i_{\max }}=\varepsilon
$$

En d'autres termes, $\left\{n+1-i_{\max }, n+1+i_{\max }\right\}$ est l'image de $\left\{b_{0}, 2 n+1\right\}$ par $w$.

Lemme 7.1. - Soit $w \in\left[W / W^{L}\right]$ tel que $\chi=w \eta$. Alors $w$ envoie

- $\left[a_{i_{\max }-1}, a_{-i_{\max }+1}\right]$ sur $\left[n+2-i_{\max }, n+i_{\max }\right]$, de façon croissante;

$-\left[a_{h}, a_{i_{\max }}\right] \cup\left[b_{k}, b_{1}\right]$ sur $\left[1, n-i_{\max }\right]$;

$-\left[a_{-i_{\max }}, a_{-h}\right] \cup\left[b_{-1}, b_{-k}\right]$ sur $\left[n+i_{\max }+2,2 n+1\right]$.

Démonstration. - Supposons que parmi les $\chi_{n+i+1}, i \in\left[i_{\max }-1,-i_{\max }+1\right]$, il y a un terme de la forme $j+\varepsilon$, avec $j \neq 0$, disons $\chi_{n+i+1}=j+\varepsilon$. On a donc $w\left(b_{j}\right)=n+i+1$. Par symétrie, on a : $\chi_{n-i+1}=-j+\varepsilon$ et $w\left(b_{-j}\right)=n-i+1$. Comme $w$ est croissant sur le segment $I(k), w\left(b_{0}\right) \in\left[w\left(b_{j}\right), w\left(b_{-j}\right)\right]=[n+i+1, n-i+1]$. Mais cela contredit la définition de $i_{\max }$. Les termes $\chi_{i}, i \in\left[i_{\max }-1,-i_{\max }+1\right]$ sont donc de la forme $l$, $l$ entier. En d'autres termes l'intervalle $\left[n+2-i_{\max }, n+i_{\max }\right]$ est l'image par $w$ d'un sous-ensemble de $I(h)$, donc un sous-intervalle de longueur $2 i_{\max }-1$ de celui-ci. Par la symétrie de $\chi$, c'est l'intervalle $\left[a_{i_{\max }-1}, a_{-i_{\max }+1}\right]$.

Montrons le deuxième point. Comme $w$ est croissant sur $I(h), w\left(\left[a_{h}, a_{i_{\max }}\right]\right)$ est majoré par $w\left(a_{i_{\max }-1}\right)=n+2-i_{\max }$. Puisque l'image réciproque de $n+1-i_{\max }$ est soit $b_{0}$ soit $2 n+1$, on a $w\left(\left[a_{h}, a_{i_{\max }}\right]\right) \subset\left[1, n-i_{\max }\right]$. Un raisonnement semblable montre que $w\left(\left[b_{k}, b_{1}\right]\right) \subset\left[1, n-i_{\max }\right]$. Donc $\left[a_{h}, a_{i_{\max }}\right] \cup\left[b_{k}, b_{1}\right] \subset\left[1, n-i_{\max }\right]$. Un comptage élémentaire d'éléments montre alors que cette inclusion est une égalité, d'où le deuxième point.

Le dernier point se démontre de façon similaire. 


\section{COROllaire 7.2}

(1) Pour tout $i \in\left[i_{\max }-1,-i_{\max }+1\right]$, on a $\chi_{n+i+1}=-i$.

(2) La dimension de $V_{U}[\chi]$ est égale à 2 .

7.1.3. Construction de $\varrho$. - On note $\mathbf{Q}=\mathbf{M}_{\mathbf{Q}} \mathbf{N}_{\mathbf{Q}}$ le sous-groupe parabolique standard $\theta$-stable de $\mathbf{G L}(\mathbf{2 n}+\mathbf{1})$ associé à la partition $\left(1, \ldots, 1,2 i_{\max }+1,1, \ldots, 1\right)$. Posons :

$$
\varrho=\chi_{1} \otimes \cdots \otimes \chi_{n-i_{\max }} \otimes\left(\xi \mathrm{St}_{1} \times \mathrm{St}_{2 i_{\max }-1} \times \xi \mathrm{St}_{1}\right) \otimes \chi_{n+2+i_{\max }} \otimes \cdots \otimes \chi_{2 n+1}
$$

C'est une représentation lisse, irréductible de $M_{Q}$. Notons $E$ son espace.

\section{Proposition 7.3}

(1) La représentation @ s'identifie à une unique sous-représentation de $\pi_{N_{Q}}$.

(2) L'opérateur A, ou plus précisément l'opérateur de $V_{N_{Q}}$ déduit de $A$, préserve $E$ et définit un opérateur d'entrelacement entre $\varrho$ et $\varrho^{\theta}$.

(3) Avec les notations usuelles, l'espace $E_{U}$ contient $V_{U}[\chi]$, en fait on a l'égalité :

$$
E_{U}[\chi]=V_{U}[\chi]
$$

Démonstration. - Notons $Z$ le centre de $M_{Q}$ que l'on identifie de façon évidente à $\left(F^{\times}\right)^{2 n-2 i_{\max }+1}$. Montrons que $\varrho$ est l'unique composante dans la filtration de Bernstein-Zelevinsky de $\pi_{N_{Q}}$ sur laquelle $Z$ agit par le caractère $\bar{\chi}=\left(\chi_{1}, \ldots\right.$, $\left.\chi_{n-i_{\max }}, 0, \chi_{n+i_{\max }}, \ldots, \chi_{2 n+1}\right)$. Soit $w$ un élément de $\left[W^{M_{Q}} \backslash W / W^{L}\right]$. Dire que $Z$ agit par le caractère $\bar{\chi}$ sur la composante correspondante à $w$ dans la filtration de Bernstein-Zelevinsky de $\pi_{N_{Q}}$ c'est dire que

- Pour tout $i \in\left[1, n-i_{\max }\right] \cup\left[n+i_{\max }+2,2 n+1\right], w \eta_{i}=\chi_{i}$;

$-\sum_{i=n-i_{\max }+1}^{n+i_{\max }+1} w \eta_{i}=0 \quad \bmod 2 \varepsilon$.

Le caractère $\bar{\chi}$ étant fixé, les éléments $w \eta_{i}, i \in\left[1, n-i_{\max }\right] \cup\left[n+i_{\max }+2,2 n+1\right]$, sont déterminés par ce qui précède. D'après le lemme 7.1, $w$ envoie bijectivement $\left[a_{h}, a_{i_{\max }}\right] \cup\left[b_{k}, b_{1}\right]$ sur $\left[1, n-i_{\max }\right]$. Puisque $\eta_{i} \neq \eta_{j}$ si $i, j \in\left[a_{h}, a_{i_{\max }}\right] \cup\left[b_{k}, b_{1}\right]$, $i \neq j$, cela détermine $w$ sur $\left[a_{h}, a_{i_{\max }}\right] \cup\left[b_{k}, b_{1}\right]$. De façon similaire, $w$ est uniquement déterminé par $\bar{\chi} \operatorname{sur}\left[a_{-i_{\max }}, a_{-h}\right] \cup\left[b_{1}, b_{-k}\right]$.

D'après ce qui précède, $w$ envoie donc $\left[a_{i_{\max }-1}, a_{-i_{\max }+1}\right] \cup\left\{b_{0}, 2 n+1\right\}$ sur $\left[n+1-i_{\max }, n+1+i_{\max }\right]$. L'hypothèse que $w \in\left[W^{M_{Q}} \backslash W / W^{L}\right]$ montre alors que $w$ envoie $\left[a_{i_{\max }-1}, a_{-i_{\max }+1}\right]$ sur $\left[n+1-i_{\max }, n-1+i_{\max }\right], b_{0}$ sur $n+i_{\max }$ et $2 n+1$ sur $n+1+i_{\max }$. Autrement dit, l'élément $w$ est uniquement déterminé par $\bar{\chi}$. La composante correspondante est égale à

$$
\chi_{1} \otimes \cdots \otimes \chi_{n-i_{\max }} \otimes\left(\mathrm{St}_{2 i_{\max }-1} \times \xi \mathrm{St}_{1} \times \xi \mathrm{St}_{1}\right) \otimes \chi_{n+2+i_{\max }} \otimes \cdots \otimes \chi_{2 n+1}
$$

qui est isomorphe à $\varrho$. Par unicité de $w, \varrho$ est facteur direct dans $\pi_{N_{Q}}$, ce qui prouve le premier point. On remarque que le caractère $\bar{\chi}$ est $\theta$-stable, cela implique le deuxième point. Enfin, un calcul simple sur la filtration de Bernstein-Zelevinsky de $\varrho_{U}$ montre 
que la dimension de $E_{U}[\chi]$ est égale à 2 , c'est-à-dire égale à celle de $V_{U}[\chi]$. D'où le résultat.

Identifions maintenant $\varrho$ à une sous-représentation de $\operatorname{Ind}_{B}^{M_{Q}}(\chi)$. L'espace de représentation $E$ de $\varrho$ se réalise comme un espace de fonctions complexes sur $M_{Q}$, de sorte que l'application $f \mapsto f^{\theta}$ soit bien définie sur $E$. L'action de $A$ sur $E$ est comme on s'y attendait :

Lemme 7.4. - Pour tout $f \in E$, on a l'égalité :

$$
A(f)=f^{\theta}
$$

Démonstration. - Soient $D_{\pi}, D_{\rho}$ les droites dans $V, E$ respectivement, définies comme en 2.3. Par définition $A$ fixe $D_{\pi}$. La droite $D_{\pi}$ se projette surjectivement sur $D_{\rho}$. On en conclut que l'opérateur déduit de $A$ sur $V_{N_{Q}}$ fixe $D_{\rho}$. D'autre part, l'opérateur $f \mapsto f^{\theta}$ fixe aussi $D_{\rho}$. Donc cet opérateur coïncide avec celui déduit de $A$.

Le lemme précédent, combiné avec la proposition 7.3, montre que $V_{U}[\chi]$ muni de l'opérateur déduit de $A$ est isomorphe à $E_{U}[\chi]$ muni de l'opérateur déduit de $f \mapsto f^{\theta}$.

7.1.4. Une base $\mathcal{B}_{\chi}$ pour $E_{U}[\chi]$. - Soit $\mathbf{P}=\mathbf{M}_{\mathbf{P}} \mathbf{N}_{\mathbf{P}}$ le sous-groupe parabolique standard $\theta$-stable de $\mathbf{G L}(\mathbf{2 n}+\mathbf{1})$ associé à la partition $\left(1, \ldots, 1,2 i_{\max }-1,1, \ldots, 1\right)$. Par définition, $\varrho$ est alors l'induite de

$$
\chi_{1} \otimes \cdots \otimes \chi_{n-i_{\max }} \otimes \chi_{n+1-i_{\max }} \otimes \mathrm{St}_{2 i_{\max }-1} \otimes \chi_{n+1+i_{\max }} \otimes \chi_{n+2+i_{\max }} \otimes \cdots \otimes \chi_{2 n+1}
$$

Rappelons brièvement la filtration de Bernstein-Zelevinsky pour $\varrho_{U}$. Il existe une filtration de $T$-modules:

$$
0=E_{0} \subset E_{1} \subset \cdots \subset E_{r}=E_{U}
$$

qui est stable par $A$ (puisque $A$ est réalisé comme $f \mapsto f^{\theta}$ ). Pour tout $w \in\left[W^{M_{Q}} / W^{M_{P}}\right]$, on a défini l'entier $d(w)$ (cf. 2.2). Notons, pour $\chi$ un caractère de $T, \mathbb{C}_{\chi}$ la représentation de $T$ sur $\mathbb{C}$ via le caractère $\chi$. Pour tout entier $1 \leqslant i \leqslant r$, on a :

$$
E_{i} / E_{i-1} \simeq \oplus_{w \in\left[W^{M_{Q}} / W^{\left.M_{P}\right], d(w)=i}\right.} \mathbb{C}_{w \chi}
$$

Pour toute représentation $V$ de $T$, on a défini $V[\chi]$ le sous-espace caractéristique correspondant à $\chi$. Le foncteur $V \longrightarrow V[\chi]$ est exact. Pour tout $w \in\left[W^{M_{Q}} / W^{M_{P}}\right]$ on peut donc trouver un élément $e_{w} \in E_{i}[w \chi], i=d(w)$ tels que

$$
\varrho_{U}(t) \cdot e_{w}=w \chi(t) e_{w} \bmod E_{i-1}[w \chi]
$$

Posons $\mathcal{B}_{\chi}=\left\{e_{w} ; w \chi=\chi\right\}$. C'est une base de $E_{U}[\chi]$. Munissons-la d'un ordre tel que $e_{w}<e_{w^{\prime}}$ si $d(w)<d\left(w^{\prime}\right)$. L'action de $T$ sur $\mathcal{B}_{\chi}$ est alors triangulaire par blocs tel que chaque bloc est une matrice diagonale dont les coefficients diagonaux sont égaux à $\chi$. 
7.1.5. Action de $A$ sur $\mathcal{B}_{\chi}$. - On sait, du fait que $\varrho$ est symétrique, que l'action déduite de $A$ sur le quotient $E_{i} / E_{i-1} \simeq \oplus_{w \in\left[W^{M_{Q}} / W^{\left.M_{P}\right], d(w)=i}\right.} \mathbb{C}_{w \chi}$ vérifie les propriétés suivantes :

(1) $A: \mathbb{C}_{w \chi} \rightarrow \mathbb{C}_{\theta(w) \chi}$

(2) Lorsque $\theta(w)=w, A$ fixe $\mathbb{C}_{w \chi}$.

Maintenant, comme l'espace $E_{i}[\chi]$ a pour base $\left\{e_{w}, w \chi=\chi, d(w) \leqslant i\right\}$, il existe des nombres complexes non nuls $c_{w}$ tels que:

$$
A\left(e_{w}\right)=c_{w} e_{\theta(w)} \bmod E_{i}
$$

D'après le deuxième point de ci-dessus, si $\theta(w)=w$, alors $c_{w}=1$. Par conséquent, la matrice de $A$ dans la base $\mathcal{B}_{\chi}$, avec un ordre sur $\mathcal{B}_{\chi}$ défini comme précédemment, est une matrice triangulaire par blocs. Chaque bloc est une matrice de permutation de $w$ au sens que ses coefficients sont nuls sauf les coefficients de coordonnées $(w, \theta(w))$ qui sont égaux à $c_{w}$. On remarque que les coefficients diagonaux de $M_{i}$ sont nuls sauf pour ceux correspondants à $(w, w)$ avec $\theta(w)=w$, auxquels cas ils sont égaux à 1 .

7.1.6. Formule pour $\operatorname{tr}_{\theta} \pi_{U}[\chi]$ et $\operatorname{tr}_{\theta} \pi_{U}$. - On déduit de ce qui précède le résultat suivant :

Proposition 7.5. - Soit $f$ une fonction localement constante, à support compact sur T. On a l'identité :

$$
\operatorname{tr}_{\theta} \pi_{U}[\chi](f)=\operatorname{card}\left\{w \in\left[W^{M_{Q}} / W^{M_{P}}\right] ; w \chi=\chi, \theta(w)=w\right\} \int_{T} \chi(t) f(t) d t
$$

On peut montrer que, si on identifie de façon évidente $W^{M_{Q}}$ à $\mathfrak{S}_{2 i_{\max }+1}$, alors :

$$
\begin{aligned}
\left\{w \in\left[W^{M_{Q}} / W^{M_{P}}\right] ; w \chi=\chi, \theta(w)=w\right\} & =\left\{w \in\left[W^{M_{Q}} / W^{M_{P}}\right], w \chi=\chi\right\} \\
& =\left\{1,\left(1,2 i_{\max }+1\right)\right\}
\end{aligned}
$$

où $\left(1,2 i_{\max }+1\right)$ est la transposition entre 1 et $2 i_{\max }+1$. Par suite, on peut énoncer le résultat de la proposition précédente sous la forme :

Corollaire 7.6. - Soit $f$ une fonction localement constante, à support compact sur T. On a l'identité :

$$
\operatorname{tr}_{\theta} \pi_{U}[\chi](f)=2 \int_{T} \chi(t) f(t) d t
$$

D'où :

$$
\operatorname{tr}_{\theta} \pi_{U}(f)=2 \sum_{\chi \in \mathcal{C}(\pi)} \int_{T} \chi(t) f(t) d t
$$




\subsection{Les modules de Jacquet $\pi_{N}$ où $\mathbf{P}=\mathbf{M N} \in \mathcal{P}^{\theta-s t}$}

\subsubsection{Décomposition de $V_{N}^{K_{M}}$ en somme de sous-espaces caractéristiques}

Posons $K_{M}=K \cap M$. On sait que l'espace $V_{N}^{K_{M}}$ est filtré par l'ensemble [ $W^{M} \backslash W /$ $\left.W^{L}\right]^{0}$ (cf. 2.4.3). La composante correspondant à un élément $w \in\left[W^{M} \backslash W / W^{L}\right]^{0}$ est isomorphe à $\left(\operatorname{Ind}_{B}^{M}(w \eta)\right)^{K_{M}}$. Ce dernier espace est de dimension 1, et sur cet espace agissent $\mathcal{H}(M)$ et $A_{M}$, le centre de $M$. Pour tout caractère $\chi$ de $T$, notons $\operatorname{res}_{P}(\chi)$ le caractère de $A_{M}$ obtenu par restriction de $\chi$ à $A_{M}$. Le tore $A_{M}$ agit alors sur $\left(\operatorname{Ind}_{B}^{M}(w \eta)\right)^{K_{M}} \operatorname{par} \operatorname{res}_{P}(w \eta)$.

Pour $\bar{\chi}$ un caractère de $A_{M}$ et $(\rho, E)$ une représentation de $M$, on définit le sous espace caractéristique :

$E[\bar{\chi}]=\left\{v \in E ; \exists\right.$ un entier $i$ tel que $(\rho(m)-\bar{\chi}(m))^{i}(v)=0$ pour tout $\left.m \in A_{M}\right\}$

On peut alors décomposer $V_{N}^{K_{M}}$ en somme de sous-espaces caractéristiques :

$$
V_{N}^{K_{M}}=\oplus_{\bar{\chi}} V_{N}^{K_{M}}[\bar{\chi}]
$$

où la somme porte sur les caractères de $A_{M}$. L'opérateur $A$ agit sur $V_{N}^{K_{M}}$ et vérifie $A\left(V_{N}^{K_{M}}[\bar{\chi}]\right)=V_{N}^{K_{M}}[\overline{w \chi}]$. Notons donc $\mathcal{C}_{P}(\pi)$ l'ensemble des caractères $\bar{\chi}$ de $A_{M}$ vérifiant les propriétés suivantes :

(1) Il existe un $w \in\left[W^{M} \backslash W / W^{L}\right]^{0}$ tel que $\bar{\chi}=\operatorname{res}_{P}(w \eta)$;

(2) $\bar{\chi}$ est $\theta$-stable, i.e. $\theta(\bar{\chi})=\bar{\chi}$.

REMARQue 7.7. - La deuxième condition ne signifie pas que $w \eta$ est $\theta$-invariant.

Pour tout $f \in \mathcal{H}(M)$, on a la formule suivante :

$$
\operatorname{tr}_{\theta} \pi_{N}(f)=\sum_{\bar{\chi} \in \mathcal{C}_{P}(\pi)} \operatorname{tr}_{\theta} \pi_{N}[\bar{\chi}](f)
$$

Les définitions des termes sont similaires à celles du cas $\mathbf{P}=\mathbf{B}$. Fixons un caractère $\bar{\chi} \in \mathcal{C}_{P}(\pi)$, et calculons $\operatorname{tr}_{\theta} \pi_{N}[\bar{\chi}]$

7.2.2. Définition de $i_{P, \max }$ - - Soit $\alpha=\left(\alpha_{1}, \ldots, \alpha_{2 r-1}\right)$ la partition associée à $\mathbf{P}$. On a : $\alpha_{1}+\cdots+\alpha_{2 r-1}=2 n+1$, et pour $i=1, \ldots, 2 r-1, \alpha_{i}=\alpha_{2 r-i}$. Posons, pour $i=1, \ldots, 2 r-1, J\left(\alpha_{i}\right)=\left[\alpha_{1}+\cdots+\alpha_{i-1}+1, \alpha_{1}+\cdots+\alpha_{i}\right]$. Les intervalles d'entiers $J\left(\alpha_{i}\right)$ forment donc une partition de $I(2 n+1)$. Identifions $W$ au groupe des permutations de $I(2 n+1)$. Soit $w$ un élément de $W$. On a quelques propriétés élémentaires suivantes :

(1) Dire que $w \in\left[W^{M} \backslash W / W^{L}\right]$ c'est dire que $w$ est croissant sur chaque intervalle $I(h), I(k)$ ainsi que $w^{-1}$ est croissant sur chaque intervalle $J\left(\alpha_{i}\right)$;

(2) Dire que $w \in\left[W^{M} \backslash W / W^{L}\right]^{0}$, c'est dire qu'en plus, les ensembles $w(I(h)) \cap$ $J\left(\alpha_{i}\right), w(I(k)) \cap J\left(\alpha_{i}\right)$ sont soit vides soit des singletons ; 
(3) Dire que $\bar{\chi}=\operatorname{res}_{P}(w \eta)$ est $\theta$-invariant, c'est dire que pour tout $i=1, \ldots, 2 r-1$, on a l'égalité :

$$
\sum_{j \in J\left(\alpha_{i}\right)}(w \eta)_{j}=-\sum_{j \in J\left(\alpha_{2 r-i}\right)}(w \eta)_{j} \bmod 2 \varepsilon
$$

Remarque 7.8. - Comme conséquence de la deuxième propriété, pour que $V_{N}^{K_{M}}$ soit non nul, il faut que $\alpha_{i} \leqslant 2$ pour tout $i$ sauf $\alpha_{r} \leqslant 3$. On va donc imposer cette condition sans perte de généralité. Sous cette hypothèse, on peut préciser légèrement la dernière propriété :

Lemme 7.9. - Pour $i=1, \ldots 2 r-1$ on a l'égalité d'ensembles modulo $2 \varepsilon$ :

$$
\left\{(w \eta)_{j} ; j \in J\left(\alpha_{i}\right)\right\}=\left\{-(w \eta)_{j} ; j \in J\left(\alpha_{2 r-i}\right)\right\}
$$

Ce lemme est facile, il est analogue à un autre résultat (lemme 8.13) que nous démontrerons.

\section{Corollaire 7.11}

(1) Il existe un caractère $\chi \in \mathcal{C}(\pi)$ tel que $\bar{\chi}=\operatorname{res}_{P}(\chi)$.

(2) Un tel caractère est conjugué à wn par un élément de $W^{M}$, où $w$ est un élément de $\left[W^{M} \backslash W / W^{L}\right]^{0}$ tel que $\operatorname{res}_{P}(w \eta)=\bar{\chi}$.

REMARQue 7.12. - Le deuxième point du corollaire ci-dessus montre que les ensembles $\left\{(w \eta)_{j} ; j \in J\left(\alpha_{i}\right)\right\}$ ne dépendent pas de $w$.

D'après le résultat ci-dessus, si $l$ est tel que $\left\{(w \eta)_{j} ; j \in I\left(\alpha_{r-l}\right)\right\}$ contient $\varepsilon$ alors d'après le lemme $7.9,\left\{(w \eta)_{j} ; j \in I\left(\alpha_{r+l}\right)\right\}$ contient $\varepsilon$ aussi. Un tel $l$ existe car il y a exactement deux $\varepsilon$ parmi les $\eta_{j}$, à savoir $\eta_{2 h+k+2}$ et $\eta_{2 n+1}$. Supposons que $l$ positif, il est alors unique.

LEMME 7.13. - On $a$ :

(1) $l=0$ si et seulement si $\alpha_{r}=3$;

(2) Si $l \neq 0$, alors $\alpha_{r-l+1}=\cdots=\alpha_{r+l-1}=1$, et si w est un élément de $\left[W^{M} \backslash W /\right.$ $\left.W^{L}\right]^{0}$ tel que $\operatorname{res}_{P}(w \eta)=\bar{\chi}$ alors $(w \eta)_{n+j}=j$ pour tout $-l<j<l$;

(3) $\operatorname{dim} V_{N}^{K_{M}}[\bar{\chi}]= \begin{cases}1 & \text { si } l=0 \\ 2 & \text { si } l>0\end{cases}$

Démonstration. - Le premier point découle du lemme 7.10. Montrons le deuxième point. Soit $w \in\left[W^{M} \backslash W / W^{L}\right]^{0}$ tel que $\operatorname{res}_{P}(w \eta)=\bar{\chi}$. Supposons qu'il existe un $m,-l<m<l$ tel que $\alpha_{r-m}=\alpha_{r+m}=2$. On a : $J\left(\alpha_{r-m}\right)=\left\{w\left(a_{i}\right), w\left(b_{j}\right)\right\}$ pour certains $i, j$. D'après le lemme 7.10, on a : $J\left(\alpha_{r+m}\right)=\left\{w\left(a_{-i}\right), w\left(b_{-j}\right)\right\}$. Donc, $\left\{w\left(b_{j}\right), w\left(b_{-j}\right)\right\} \subset J\left(\alpha_{r-m}\right) \cup J\left(\alpha_{r+m}\right) \subset \cup_{m^{\prime}=-l+1}^{l-1} J\left(\alpha_{r-m^{\prime}}\right)$, qui est un intervalle. Donc $w\left(\left[b_{j}, b_{-j}\right]\right) \subset \cup_{m^{\prime}=-l+1}^{l-1} J\left(\alpha_{r-m^{\prime}}\right)$. Cela implique que $w\left(b_{0}\right) \in$ $\cup_{m^{\prime}=-l+1}^{l-1} J\left(\alpha_{r-m^{\prime}}\right)$. Mais $w\left(b_{0}\right) \in J\left(\alpha_{r-l}\right) \cup J\left(\alpha_{r+l}\right)$, c'est donc impossible. Donc, $\alpha_{r-l+1}=\cdots=\alpha_{r+l-1}=1$. Un raisonnement similaire montre alors que 
$[n-l+1, n+l-1]$ est l'image d'un sous-intervalle $I(h)$, donc un sous-intervalle de longueur $2 l-1$. Par symétrie, c'est $\left[a_{l-1}, a_{-l+1}\right]$. D'où le deuxième point. Démontrons le dernier point. Par la filtration de Bernstein-Zelevinsky, la dimension de $\operatorname{dim} V_{N}^{K_{M}}[\bar{\chi}]$ est égal au cardinal de

$$
\left\{w \in\left[W^{M} \backslash W / W^{L}\right]^{0} ; \operatorname{res}_{P}(w \eta)=\bar{\chi}\right\}
$$

Soit $w$ un tel élément. Les ensembles $\left\{(w \eta)_{j} ; j \in J\left(\alpha_{i}\right)\right\}$ ne dépendent que de $\bar{\chi}$. Notons-les $\left\{\chi_{j} ; j \in J\left(\alpha_{i}\right)\right\}$. Montrons que $w_{\mid I(h)}$ est uniquement déterminé par ces ensembles. En effet, soit $a_{m} \in I(h)$, il existe alors un unique $i$ tel que $m \in\left\{\chi_{j} ; j \in J\left(\alpha_{i}\right)\right\}$. Distinguons trois cas :

(1) $\alpha_{i}=1, J\left(\alpha_{i}\right)=\{j\}$. On a :

$$
(w \eta)_{j}=\chi_{j}=m=\eta_{a_{m}}
$$

Donc, $w\left(a_{m}\right)=j$.

(2) $\alpha_{i}=2, J\left(\alpha_{i}\right)=\{j, j+1\}$. On a :

$$
\left\{(w \eta)_{j},(w \eta)_{j+1}\right\}=\left\{\chi_{j}, \chi_{j+1}\right\}=\left\{m, m^{\prime}+\varepsilon\right\}
$$

Comme $w^{-1}$ est croissant sur $\{j, j+1\}$, ces égalités impliquent que $w\left(a_{m}\right)=j$.

(3) $\alpha_{i}=3$, donc $i=r, m=0$, et $J\left(\alpha_{r}\right)=\{n, n+1, n+2\}$. D'après le lemme 7.10 , on a :

$$
\left\{(w \eta)_{n},(w \eta)_{n+1}(w \eta)_{n+2}\right\}=\{0, \varepsilon, \varepsilon\}=\left\{\eta_{a_{0}}, \eta_{b_{0}}, \eta_{2 n+1}\right\}
$$

Par la croissance de $w^{-1}$ sur $\{n, n+1, n+2\}$, on en déduit $w\left(a_{0}\right)=n, w\left(b_{0}\right)=n+1$, $w(2 n+1)=n+2$.

On montre de façon similaire que $w_{\mid\left[b_{k}, b_{1}\right]}, w_{\mid\left[b_{-1}, b_{-k}\right]}$ sont uniquement déterminés par les ensembles $\left\{\chi_{j} ; j \in J\left(\alpha_{i}\right)\right\}$.

Quant à $w\left(b_{0}\right), w\left(a_{0}\right)$, si $l=0$, d'après ce qui précède, on a $w\left(b_{0}\right)=n+1$, $w(2 n+1)=n+2$. Si $l>0$, on a deux possibilités : soit $w\left(b_{0}\right) \in J\left(\alpha_{r-l}\right), w(2 n+1) \in$ $J\left(\alpha_{r+l}\right)$, soit $w\left(b_{0}\right) \in J\left(\alpha_{r+l}\right), w(2 n+1) \in J\left(\alpha_{r-l}\right)$. On montre par un raisonnement similaire que ces deux cas peuvent se produire et donnent deux choix pour $w_{\mid\left\{b_{0}, 2 n+1\right\}}$. Le lemme en découle.

Posons :

$$
i_{P, \max }= \begin{cases}1, & \text { si } l=0 \\ \alpha_{r+1}+\cdots+\alpha_{r+l}, & \text { si } l>0\end{cases}
$$

7.2.3. Construction de $\varrho$. - Soit $\mathbf{Q}=\mathbf{M}_{\mathbf{Q}} \mathbf{N}_{\mathbf{Q}}$ le sous-groupe parabolique standard $\theta$-stable associé à la partition $\left(\alpha_{1}, \ldots, \alpha_{r-l-1}, 2 i_{P, \max }+1, \alpha_{r+l+1}, \ldots, \alpha_{2 r-1}\right)$. On a : $\mathbf{P} \subset \mathbf{Q}$. Considérons la composante correspondante à $w$ dans la filtration de Bernstein-Zelevinsky de $\pi_{N_{Q}}$. Elle est de la forme : $\pi_{1} \otimes \cdots \otimes \pi_{2 r-1}$. Posons :

$$
\varrho=\pi_{1} \otimes \cdots \otimes \pi_{r-l-1} \otimes\left(\xi \mathrm{St}_{1} \times \mathrm{St}_{2 i_{P, \max }-1} \times \xi \mathrm{St}_{1}\right) \otimes \pi_{r+l+1} \otimes \cdots \otimes \pi_{2 r-1}
$$

et notons $E$ son espace. On a le résultat suivant, qui est analogue à celui pour $\mathbf{P}=\mathbf{B}$ : 


\section{Proposition 7.14}

(1) La représentation @ s'identifie à une sous-représentation de $\pi_{N_{Q}}$.

(2) L'opérateur A conserve $E$ et définit un opérateur d'entrelacement entre $\varrho$ et $\varrho^{\theta}$.

(3) On a l'égalité : $E_{N}^{K_{M}}[\bar{\chi}]=V_{N}^{K_{M}}[\bar{\chi}]$.

Par construction, $\varrho$ est symétrique. Réalisons $E$ comme un espace de fonctions sur $M_{Q}$ de sorte que l'application $f \mapsto f^{\theta}$ soit bien définie et entrelace $\varrho$ et $\varrho^{\theta}$. Le lemme suivant est similaire à un résultat du cas $\mathbf{P}=\mathbf{B}$ :

Lemme 7.15. - La restriction de $A$ à $E$ est de la forme : $A(f)=f^{\theta}$.

7.2.4. Formule pour $\operatorname{tr}_{\theta} \pi_{N}[\bar{\chi}]$. - Pour $f \in \mathcal{H}(M)$, avec les mêmes arguments que dans le cas $\mathbf{P}=\mathbf{B}$, on montre que :

Proposition 7.16. - Pour tout $f \in \mathcal{H}(M)$, on a l'égalité :

$$
\operatorname{tr}_{\theta} \pi_{N}[\bar{\chi}](f)=\operatorname{card}\left\{w \in\left[W^{M} \backslash W^{M_{Q}}\right] ; \operatorname{res}_{P}(w \chi)=\bar{\chi}, \theta(w)=w\right\} \cdot \operatorname{tr} \operatorname{Ind}_{B}^{M}(\chi)(f)
$$

Le terme $\operatorname{card}\left\{w \in\left[W^{M} \backslash W^{M_{Q}}\right] ; \operatorname{res}_{P}(w \chi)=\bar{\chi}, \theta(w)=w\right\}$ dans la formule précédente est égal à 1 ou 2 selon que $l=0$ ou $l \neq 0$. On en déduit :

$$
\operatorname{card}\left\{w \in\left[W^{M} \backslash W^{M_{Q}}\right] ; \operatorname{res}_{P}(w \chi)=\bar{\chi}, \theta(w)=w\right\}=\operatorname{dim} V_{N}^{K_{M}}[\bar{\chi}]
$$

On obtient :

Corollaire 7.17. - Avec les mêmes notations que dans la proposition précédente, on a l'identité :

$$
\operatorname{tr}_{\theta} \pi_{N}[\bar{\chi}](f)=\operatorname{dim} V_{N}^{K_{M}}[\bar{\chi}] \operatorname{tr} \operatorname{Ind}_{B}^{M}(\chi)(f)
$$

REMARQue 7.18. - On sait que

$$
\operatorname{dim} V_{N}^{K_{M}}[\bar{\chi}]=\operatorname{card}\left\{w \in\left[W^{M} \backslash W / W^{L}\right]^{0} ; \operatorname{res}_{P}(w \eta)=\bar{\chi}\right\} .
$$

On sait aussi que si $w \in\left[W^{M} \backslash W / W^{L}\right]^{0}$ t.q. $\operatorname{res}_{P}(w \eta)=\bar{\chi}$, alors $w \eta$ est conjugué à $\chi$ par un élément de $W^{M}$. Ainsi, si $f \in \mathcal{H}(M)$, on a une autre façon d'exprimer le résultat obtenu :

$$
\operatorname{tr}_{\theta} \pi_{N}[\bar{\chi}](f)=\operatorname{tr} \pi_{N}[\bar{\chi}](f)=\sum_{\substack{w \in\left[W^{M} \backslash W / W^{L}\right]^{0} \\ \operatorname{res}_{P}(w \eta)=\bar{\chi}}} \operatorname{tr}_{B} \operatorname{Ind}_{B}^{M}(w \eta)(f)
$$

\subsection{Calculs explicites de $\operatorname{tr}_{\theta-c} \pi$}

D'après ce qui précède, on $\mathrm{a}$ :

Proposition 7.19. - Pour $\mathbf{P}=\mathbf{M N} \in \mathcal{P}^{\theta-s t}$, et $\bar{\chi} \in \mathcal{C}_{P}(\pi)$, soit $\chi \in \mathcal{C}(\pi)$ un élément de restriction $\bar{\chi}$. Pour tout $f \in \mathcal{H}(G)$, on a l'identité :

$$
\operatorname{tr}_{\theta-c} \pi(f)=\sum_{\mathbf{P}=\mathbf{M N} \in \mathcal{P}^{\theta-s t}} \sum_{\bar{\chi} \in \mathcal{C}_{P}(\pi)}(-1)^{a_{P}^{\theta}-a_{G}^{\theta}} \operatorname{dim} V_{N}^{K_{M}}[\bar{\chi}] \operatorname{tr} \operatorname{Ind}_{B}^{M}(\chi)\left(\widehat{\chi}_{N}^{\theta} f^{P}\right)
$$


7.3.1. Une section pour $\chi \longmapsto \operatorname{res}_{P}(\chi)$. — Le but de ce paragraphe est de récrire la formule 7.20 en une somme paramétrée par les éléments $\chi \in \mathcal{C}(\pi)$. Soit $\mathbf{P}=\mathbf{M N} \in$ $\mathcal{P}^{\theta-s t}$ et soit $\bar{\chi} \in \mathcal{C}_{P}(\pi)$. D'après le corollaire 7.11 , il existe un caractère $\chi \in \mathcal{C}(\pi)$ de restriction $\bar{\chi}$. Cependant, un tel caractère n'est pas unique, nous allons donc fixer un choix pour $\chi$, ou ce qui revient au même, construire une $\operatorname{section} \sec _{P}$ de l'application :

$$
\begin{aligned}
\operatorname{res}_{P}: \mathcal{C}(\pi) & \longrightarrow \mathcal{C}_{P}(\pi) \\
\chi & \longmapsto \operatorname{res}_{P}(\chi)
\end{aligned}
$$

Le caractère $\bar{\chi}$ étant donné, soit $\chi=\left(\chi_{1}, \ldots, \chi_{2 n+1}\right)$ un élément de $\mathcal{C}(\pi)$ tel que $\operatorname{res}_{P}(\chi)=\bar{\chi}$. Soit $\alpha=\left(\alpha_{1}, \ldots, \alpha_{2 r-1}\right)$ la partition correspondante à $\mathbf{P}$. Les intervalles $I_{\alpha_{i}}$ ont été définis précédemment. Sans perte de généralité, on suppose toujours que pour $i=1, \ldots, 2 r-1, i \neq r, \alpha_{i} \leqslant 2$, et $\alpha_{r} \leqslant 3$. Soit $i \in[1, r]$.

- Si $\alpha_{i}=1$, écrivons $I\left(\alpha_{i}\right)=\{j\}$, et posons :

$$
\zeta_{j}=\chi_{j}
$$

- Si $\alpha_{i}=2$, écrivons $I\left(\alpha_{i}\right)=\{j, j+1\}$. On a : $\left\{\chi_{j}, \chi_{j+1}\right\}=\{a, b+\varepsilon\}$ pour certains $a, b$, avec $a \in[h,-h], b \in[k,-k]$. Posons alors :

$$
\begin{array}{ll}
\zeta_{j}=a, \zeta_{j+1}=b+\varepsilon & \text { si } a<b \\
\zeta_{j}=b+\varepsilon, \zeta_{j+1}=a & \text { si } a \geqslant b
\end{array}
$$

- Si $\alpha_{r}=3$, alors $I\left(\alpha_{r}\right)=\{n, n+1, n+2\}$ et $\chi_{n}=\varepsilon, \chi_{n+1}=0, \chi_{n+2}=\varepsilon$. Posons simplement :

$$
\zeta_{n}=\varepsilon, \zeta_{n+1}=0
$$

La construction ci-dessus définit des éléments $\zeta_{i}, i=1, \ldots, n+1$. Pour $i=$ $n+2, \ldots, 2 n+1$, on pose tout simplement :

$$
\zeta_{i}=-\zeta_{2 n+2-i} \quad \bmod 2 \varepsilon
$$

Posons $\zeta=\left(\zeta_{1}, \zeta_{2}, \ldots, \zeta_{2 n+1}\right)$. Ce caractère non ramifié de $T$ est un élément de $\mathcal{C}(\pi)$. En plus, il ne dépend que de $\bar{\chi}$. On peut donc définir :

$$
\sec _{P}(\bar{\chi})=\zeta
$$

7.3.2. Réécriture de la formule 7.20. — On peut récrire la formule 7.20 sous la forme suivante :

$$
\operatorname{tr}_{\theta-c} \pi(f)=\sum_{\chi \in \mathcal{C}(\pi)} \operatorname{tr}_{\theta-c} \pi[\chi](f)
$$

où

$$
\operatorname{tr}_{\theta-c} \pi[\chi](f)=\sum_{\substack{\mathbf{P}=\mathbf{M N} \in \mathcal{P}^{\theta-s t} \\ \bar{\chi} \in \mathcal{C}_{P}(\pi), \sec P(\bar{\chi})=\chi}}(-1)^{a_{P}^{\theta}-a_{G}^{\theta}} \operatorname{dim} V_{N}^{K_{M}}[\bar{\chi}] \operatorname{tr} \operatorname{Ind}_{B}^{M}(\chi)\left(\widehat{\chi}_{N}^{\theta} f^{P}\right)
$$




\subsubsection{Formule explicite pour $\operatorname{tr}_{\theta-c} \pi[\chi](f)$}

Fixons donc un $\chi=\left(\chi_{1}, \chi_{2}, \ldots, \chi_{2 n+1}\right) \in \mathcal{C}(\pi)$ et une fonction $f \in \mathcal{H}(G)$. Notons $\Sigma$ l'ensemble des entiers $i \in[1, n]$ satisfaisant aux conditions suivantes :

(1) $\chi_{i}-\chi_{i+1} \notin \mathbb{R}$;

(2) $\operatorname{Re}\left(\chi_{i}\right) \leqslant \operatorname{Re}\left(\chi_{i+1}\right)$, et si $\operatorname{Re}\left(\chi_{i}\right)=\operatorname{Re}\left(\chi_{i+1}\right)$, alors $\chi_{i} \in \mathbb{R}+\varepsilon, \chi_{i+1} \in \mathbb{R}$.

Lemme 7.21. - Lorsque $i$ parcourt $\Sigma$, les ensembles $\{i, i+1\}$ sont disjoints.

Démonstration. - Soit $w \in\left[W / W^{L}\right]$ tel que $w \eta=\chi$. Soit $i \in \Sigma$. Supposons que $\chi_{i}=m, \chi_{i+1}=m^{\prime}+\varepsilon$ avec $m \leqslant m^{\prime}$. Si $i+1 \in \Sigma$ alors $\chi_{i+2}=m^{\prime \prime}$ avec $m^{\prime} \leqslant m^{\prime \prime}$, donc $m \leqslant m^{\prime \prime}$. On a alors $w\left(a_{m}\right)=i, w\left(a_{m^{\prime \prime}}\right)=i+2(c f$. paragraphe 7.1.2 pour les définitions), mais cela contredit la propriété de croissance de $w$ sur $I(h)$.

Maintenant, supposons que $\chi_{i}=m+\varepsilon, \chi_{i+1}=m^{\prime}$ avec $m \leqslant m^{\prime}$. Considérons d'abord le cas où $m=0$. On a donc $i=n+1-i_{\max }$. Si $i_{\max }=1, i=n$ et il n'y a rien à démontrer. Si $i_{\max }>1$, alors d'après le corollaire 7.2, $\chi_{i+1}=i_{\max }-1$, $\chi_{i+2}=i_{\max }-2$ et $i+1$ ne peut appartenir à $\Sigma$. Supposons maintenant que $m \neq 0$, donc $m>0$ d'après le lemme 7.1. Si $i+1 \in \Sigma$, alors $\chi_{i+2}=m^{\prime \prime}+\varepsilon$ avec $m^{\prime}<m^{\prime \prime}$. On a $0<m<m^{\prime \prime}$, donc $w\left(b_{m}\right)=i, w\left(b_{m^{\prime \prime}}\right)=i+2$, mais cela contredit la propriété de croissance de $w$ sur $I(k)$. Le lemme est démontré.

Grâce à ce résultat, pour $\Delta \subset \Sigma$, on peut associer à $\Delta$ une partition de $2 n+1$ dont les parts sont d'une des formes suivantes :

- Si $n \notin \Delta$ :

$-\{n+1\},\{i\},\{2 n+2-i\}, i=1, \ldots, n$, et $i \notin \Delta$;

$-\{i, i+1\},\{2 n+1-i, 2 n+2-i\}, i \in[1, n-1] \cap \Delta$.

- Si $n \in \Delta$ :

$-\{i\},\{2 n+2-i\}, i=1, \ldots, n$, et $i \notin \Delta$;

$-\{i, i+1\},\{2 n+1-i, 2 n+2-i\}, i \in[1, n-1] \cap \Delta ;$

$-\{n, n+1, n+2\}$.

Notons $\mathbf{P}_{\Delta}$ le sous-groupe parabolique standard $\theta$-stable correspondant. Les couples $\mathbf{P}=\mathbf{M N}, \bar{\chi}$ tels que $\sec _{P}(\bar{\chi})=\chi$ sont exactement les $\mathbf{P}_{\Delta}, \operatorname{res}_{P_{\Delta}}(\chi)$. On a l'égalité :

$$
\operatorname{tr}_{\theta-c} \pi[\chi](f)=\sum_{\Delta \subset \Sigma}(-1)^{a_{P_{\Delta}}^{\theta}-a_{G}^{\theta}} \operatorname{dim} V_{N_{\Delta}}^{K_{M_{\Delta}}}[\bar{\chi}] \operatorname{tr} \operatorname{Ind}_{B}^{M_{\Delta}}(\chi)\left(\hat{\chi}_{N_{\Delta}}^{\theta} f^{P_{\Delta}}\right)
$$

C'est sous cette forme que l'on va calculer explicitement $\operatorname{tr}_{\theta-c} \pi[\chi](f)$. En effet, on a les égalités :

$$
\begin{gathered}
a_{P_{\Delta}}^{\theta}-a_{G}^{\theta}=\operatorname{card}(\Delta)+n \\
\operatorname{dim} V_{N_{\Delta}}^{K_{M_{\Delta}}}[\bar{\chi}]=\left[\begin{array}{ll}
2 & \text { si } n \notin \Delta \\
1 & \text { si } n \in \Delta
\end{array}\right.
\end{gathered}
$$


Définissons le sous-ensemble $\mathcal{M}(\Delta)$ de $\mathbb{Z}^{2 n+1}$ par la formule :

$$
\mathcal{M}(\Delta)=\left\{\boldsymbol{m}=\left(m_{1}, \ldots, m_{2 n+1}\right) ; \begin{array}{l}
\text { pour tout } i=1, \ldots, n, \text { et } i \notin \Delta, \\
m_{1}+\cdots+m_{i}-m_{2 n+2-i}-\cdots-m_{2 n+1}>0
\end{array}\right\}
$$

Rappelons que l'on a fixé un $f \in \mathcal{H}(G)$. Écrivons la transformée de Satake de $f$ :

$$
S(f)\left(X_{1}, \ldots, X_{2 n+1}\right)=\sum_{\boldsymbol{m}=\left(m_{1}, \ldots, m_{2 n+1}\right) \in \mathbb{Z}^{2 n+1}} c_{\boldsymbol{m}} X_{1}^{m_{1}} \cdots X_{2 n+1}^{2 n+1}
$$

On dispose d'un lemme facile suivant :

LEMME 7.25. - La transformée de Satake de $\widehat{\chi}_{N_{\Delta}}^{\theta} f^{P_{\Delta}}$ est donnée par la formule:

$$
S\left(\widehat{\chi}_{N_{\Delta}}^{\theta} f^{P_{\Delta}}\right)\left(X_{1}, \ldots, X_{2 n+1}\right)=\sum_{\boldsymbol{m} \in \mathcal{M}(\Delta)} c_{\boldsymbol{m}} X_{1}^{m_{1}} \cdots X_{2 n+1}^{2 n+1}
$$

Posons : $\langle\chi, \boldsymbol{m}\rangle=\sum_{i=1}^{2 n+1} \chi_{i} m_{i}$. Par application de la formule de Van Dijk, on en déduit immédiatement le résultat suivant :

COROllaire 7.26. - On a l'égalité :

$$
\operatorname{tr} \operatorname{Ind}_{B}^{M_{\Delta}}(\chi)\left(\widehat{\chi}_{N_{\Delta}}^{\theta} f^{P_{\Delta}}\right)=\sum_{\boldsymbol{m} \in \mathcal{M}(\Delta)} c_{\boldsymbol{m}} q^{-\langle\chi, \boldsymbol{m}\rangle}
$$

Posons, pour $\boldsymbol{l}=\left(l_{1}, \ldots, l_{n}\right) \in \mathbb{Z}^{n}$ :

$$
\begin{gathered}
d_{\boldsymbol{l}}=\sum_{\substack{\boldsymbol{m}=\left(m_{1}, \ldots, m_{2 n+1}\right) \in \mathbb{Z}^{2 n+1} \\
\text { pour tout } i=1, \ldots n: \\
m_{i}-m_{2 n+2-i}=l_{i}}} c_{\boldsymbol{m}} \\
\langle\chi, \boldsymbol{l}\rangle=\chi_{1} l_{1}+\chi_{2} l_{2}+\cdots+\chi_{n} l_{n}
\end{gathered}
$$

et pour $\Delta \subset[1, n]$ :

$$
\mathcal{L}(\Delta)=\left\{\boldsymbol{l}=\left(l_{1}, \ldots l_{n}\right) \in \mathbb{Z}^{n} ; \text { pour tout } i \notin \Delta, l_{1}+\cdots+l_{i}>0\right\}
$$

Notons $\mathbf{1}_{\mathcal{L}(\Delta)}$ la fonction caractéristique de $\mathcal{L}(\Delta) \subset \mathbb{Z}^{n}$. Le corollaire précédent peut s'énoncer de la façon suivante :

\section{Corollaire 7.28. - On a l'égalité :}

$$
\operatorname{tr} \operatorname{Ind}_{B}^{M_{\Delta}}(\chi)\left(\widehat{\chi}_{N_{\Delta}}^{\theta} f^{P_{\Delta}}\right)=\sum_{\boldsymbol{l} \in \mathbb{Z}^{n}} d_{\boldsymbol{l}} q^{-\langle\chi, \boldsymbol{l}\rangle} \mathbf{1}_{\mathcal{L}(\Delta)}(\boldsymbol{l})
$$

Les formules 7.22 à 7.29 calculent explicitement $\operatorname{tr}_{\theta-c} \pi[\chi](f)$. On va distinguer deux cas selon que $n \in \Delta$ ou non. 
Cas $n \notin \Sigma$. - On a l'identité :

$$
\operatorname{tr}_{\theta-c} \pi[\chi](f)=2 \sum_{\boldsymbol{l} \in \mathbb{Z}^{n}}\left(\sum_{\Delta \subset \Sigma}(-1)^{\operatorname{card}(\Delta)+n} \mathbf{1}_{\mathcal{L}(\Delta)}(\boldsymbol{l})\right) d_{\boldsymbol{l}} q^{-\langle\chi, \boldsymbol{l}\rangle}
$$

Posons :

$$
\mathcal{L}^{0}(\Sigma)=\left\{l=\left(l_{1}, \ldots, l_{n}\right) \in \mathbb{Z}^{n} ; \begin{array}{l}
\text { pour tout } i=1, \ldots, n: \\
l_{1}+\cdots+l_{i} \leqslant 0 \quad \text { si } i \in \Sigma \\
l_{1}+\cdots+l_{i}>0 \text { si } i \notin \Sigma
\end{array}\right\}
$$

On a la formule combinatoire élémentaire suivante :

LEMME 7.30. - On a l'égalité :

$$
\sum_{\Delta \subset \Sigma}(-1)^{\operatorname{card}(\Delta)+n} \mathbf{1}_{\mathcal{L}(\Delta)}=(-1)^{\operatorname{card}(\Sigma)+n} \mathbf{1}_{\mathcal{L}^{0}(\Sigma)}
$$

La proposition suivante résulte alors de ce qui précède et donne une formule explicite pour $\operatorname{tr}_{\theta-c} \pi[\chi](f)$ dans le cas où $n \notin \Sigma$.

Proposition 7.31. - Supposons que $n \notin \Sigma$, alors on a l'identité :

$$
\operatorname{tr}_{\theta-c} \pi[\chi](f)=2(-1)^{\operatorname{card}(\Sigma)+n} \sum_{\boldsymbol{l} \in \mathbb{Z}^{n}} \mathbf{1}_{\mathcal{L}^{0}(\Sigma)}(\boldsymbol{l}) d_{\boldsymbol{l}} q^{-\langle\chi, \boldsymbol{l}\rangle}
$$

Cas $n \in \Sigma$. - La trace $\operatorname{tr}_{\theta-c} \pi[\chi](f)$ est égale à

$$
\sum_{\boldsymbol{l} \in \mathbb{Z}^{n}}\left(\sum_{\Delta \subset \Sigma \backslash\{n\}} 2(-1)^{\operatorname{card}(\Delta)+n} \mathbf{1}_{\mathcal{L}(\Delta)}(\boldsymbol{l})-(-1)^{\operatorname{card}(\Delta)+n} \mathbf{1}_{\mathcal{L}(\Delta \cup\{n\})}(\boldsymbol{l})\right) d_{\boldsymbol{l}} q^{-\langle\chi, \boldsymbol{l}\rangle}
$$

Posons :

$$
\begin{aligned}
& \mathcal{L}^{1}(\Sigma)=\left\{\begin{array}{l}
\text { pour tout } i=1, \ldots, n: \\
l ; l_{1}+\cdots+l_{i} \leqslant 0 \quad \text { si } i \in \Sigma \backslash\{n\} \\
l_{1}+\cdots+l_{i}>0 \quad \text { si } i \notin \Sigma \backslash\{n\}
\end{array}\right\} \\
& \mathcal{L}^{2}(\Sigma)=\left\{\begin{array}{cc}
\text { pour tout } i=1, \ldots, n-1 \\
l ; & l_{1}+\cdots+l_{i} \leqslant 0 \quad \text { si } i \in \Sigma \\
l_{1}+\cdots+l_{i}>0 & \text { si } i \notin \Sigma
\end{array}\right\}
\end{aligned}
$$

On a $: \mathcal{L}^{1}(\Sigma)=\left\{\boldsymbol{l} \in \mathcal{L}^{2}(\Sigma) ; l_{1}+\cdots+l_{n}>0\right\}$. Le lemme facile suivant est similaire au lemme 7.30 :

LEMME 7.32. - On a les égalités :

$$
\begin{aligned}
\sum_{\Delta \subset \Sigma \backslash\{n\}}(-1)^{\operatorname{card}(\Delta)+n} \mathbf{1}_{\mathcal{L}(\Delta)} & =(-1)^{\operatorname{card}(\Sigma)-1+n} \mathbf{1}_{\mathcal{L}^{1}(\Sigma)} \\
\sum_{\Delta \subset \Sigma \backslash\{n\}}(-1)^{\operatorname{card}(\Delta)+n} \mathbf{1}_{\mathcal{L}(\Delta \cup\{n\})} & =(-1)^{\operatorname{card}(\Sigma)-1+n} \mathbf{1}_{\mathcal{L}^{2}(\Sigma)}
\end{aligned}
$$

On en déduit une expression explicite pour $\operatorname{tr}_{\theta-c} \pi[\chi](f)$ dans le cas où $n \in \Sigma$ : 
Proposition 7.33. - Supposons que $n \in \Sigma$, alors on a l'identité :

$$
\operatorname{tr}_{\theta-c} \pi[\chi](f)=(-1)^{\operatorname{card}(\Sigma)-1+n} \sum_{\boldsymbol{l} \in \mathbb{Z}^{n}}\left(2 \mathbf{1}_{\mathcal{L}^{1}(\Sigma)}(\boldsymbol{l})-\mathbf{1}_{\mathcal{L}^{2}(\Sigma)}(\boldsymbol{l})\right) d_{\boldsymbol{l}} q^{-\langle\chi, \boldsymbol{l}\rangle}
$$

On peut écrire différemment la formule ci-dessus. Posons :

$$
\begin{aligned}
& \mathcal{L}^{3}(\Sigma)=\left\{\begin{array}{c}
\text { pour tout } i=1, \ldots, n-1: \\
l_{1}+\cdots+l_{i} \leqslant 0 \quad \text { si } i \in \Sigma \\
l_{1}+\cdots+l_{i}>0 \text { si } i \notin \Sigma \\
l_{1}+\cdots+l_{n-1}>-l_{n} \geqslant 0
\end{array}\right\} \\
& \mathcal{L}^{4}(\Sigma)=\left\{\begin{array}{ll}
\text { pour tout } i=1, \ldots, n-1 \\
l ; \\
l_{1}+\cdots+l_{i} \leqslant 0 & \text { si } i \in \Sigma \\
l_{1}+\cdots+l_{i}>0 & \text { si } i \notin \Sigma \\
l_{n}=0 &
\end{array}\right\}
\end{aligned}
$$

Corollaire 7.34. - Supposons que $n \in \Sigma$, alors on a l'égalité :

$$
\operatorname{tr}_{\theta-c} \pi[\chi](f)=(-1)^{\operatorname{card}(\Sigma)-1+n} \sum_{\boldsymbol{l} \in \mathbb{Z}^{n}}\left(2 \mathbf{1}_{\mathcal{L}^{3}(\Sigma)}(\boldsymbol{l})-\mathbf{1}_{\mathcal{L}^{4}(\Sigma)}(\boldsymbol{l})\right) d_{\boldsymbol{l}} q^{-\langle\chi, \boldsymbol{l}\rangle}
$$

Démonstration. - En effet, écrivons

$$
\mathcal{L}^{2}(\Sigma)=\left\{\boldsymbol{l} \in \mathcal{L}^{2}(\Sigma) ; l_{n}>0\right\} \sqcup\left\{\boldsymbol{l} \in \mathcal{L}^{2}(\Sigma) ; l_{n}<0\right\} \sqcup \mathcal{L}^{4}(\Sigma)
$$

Comme on l'a remarqué, $\chi_{n}=\varepsilon$, le simple changement de variable

$$
\boldsymbol{l}=\left(l_{1}, \ldots, l_{n-1}, l_{n}\right) \longmapsto\left(l_{1}, \ldots, l_{n-1},-l_{n}\right)
$$

donne :

On obtient :

$$
\sum_{\boldsymbol{l} \in \mathcal{L}^{2}(\Sigma), l_{n}>0} d_{l} q^{-\langle\chi, \boldsymbol{l}\rangle}=\sum_{\boldsymbol{l} \in \mathcal{L}^{2}(\Sigma), l_{n}<0} d_{l} q^{-\langle\chi, l\rangle}
$$

$$
\sum_{l \in \mathcal{L}^{2}(\Sigma)} d_{l} q^{-\langle\chi, l\rangle}=2 \sum_{\boldsymbol{l} \in \mathcal{L}^{2}(\Sigma), l_{n}>0} d_{\boldsymbol{l}} q^{-\langle\chi, l\rangle}+\sum_{\boldsymbol{l} \in \mathcal{L}^{4}(\Sigma)} d_{\boldsymbol{l}} q^{-\langle\chi, l\rangle}
$$

Mais $\mathcal{L}^{1}(\Sigma)=\left\{\boldsymbol{l} \in \mathcal{L}^{2}(\Sigma) ; l_{n}>0\right\} \sqcup \mathcal{L}^{3}(\Sigma)$, d'où le lemme.

\subsection{Transformation de Satake de $\operatorname{tr}_{\theta-c} \pi$}

Soit $f \in \mathcal{H}$, écrivons toujours :

$$
S(f)\left(X_{1}, \ldots, X_{2 n+1}\right)=\sum_{\boldsymbol{m}=\left(m_{1}, \ldots, m_{2 n+1}\right) \in \mathbb{Z}^{2 n+1}} c_{\boldsymbol{m}} X_{1}^{m_{1}} \cdots X_{2 n+1}^{2 n+1}
$$

On a l'égalité :

$$
p(S(f))\left(X_{1}, \ldots, X_{n}\right)=\sum_{l \in \mathbb{Z}^{n}} d_{l} X_{1}^{l_{1}} \cdots X_{n}^{l_{n}}
$$

où $d_{l}$ est défini au paragraphe précédent. 
7.4.1. Cas où $\Sigma$ ne contient pas $n$. - On a : $\chi_{n} \neq \varepsilon$, donc $i_{\max }>1$. D'après le corollaire 7.2 , on a $: \chi_{n}=1$.

Rappelons que l'on a posé : $\widehat{T}_{u}=\left\{\left(x_{1}, \ldots, x_{n}\right) \in \mathbb{C}^{n} ;\left|x_{1}\right|=\cdots=\left|x_{n}\right|=1\right\}$. Plus généralement, pour $\delta=\left(\delta_{1}, \ldots, \delta_{n}\right)$ un $n$-uplet de nombres réels positifs, posons :

$$
\widehat{T}_{u, \delta}=\left\{\left(x_{1}, \ldots, x_{n}\right) \in \mathbb{C}^{n} ; \text { pour tout } i=1, \ldots, n,\left|x_{i}\right|=\delta_{i}\right\}
$$

La proposition 7.31, jointe avec l'égalité 7.35 et la formule d'orthogonalité 3.20, donne :

$$
\begin{aligned}
& \operatorname{tr}_{\theta-c} \pi[\chi](f)=2(-1)^{\operatorname{card}(\Sigma)+n} \int_{\widehat{T}_{u, \delta}} p(S(f))\left(x_{1}, \ldots, x_{n}\right) \\
& \cdot\left(\sum_{l \in \mathcal{L}^{0}(\Sigma)} q^{-\langle\chi, l\rangle} x_{1}^{-l_{1}} \cdots x_{n}^{-l_{n}}\right) d^{\times} x_{1} \cdots d^{\times} x_{n}
\end{aligned}
$$

pourvu que cette expression converge.

On dira qu'un $n$-uplet $\delta=\left(\delta_{1}, \ldots, \delta_{n}\right)$ est bon pour $\chi$ s'il vérifie les conditions suivantes :

$$
\left\{\begin{array}{l}
\delta_{1}, \ldots, \delta_{n} \text { sont des réels assez proches de } 1 \\
\delta_{i}<\delta_{i+1} \quad \text { si } i \in \Sigma \\
\delta_{i}>\delta_{i+1} \quad \text { si } i \notin \Sigma
\end{array}\right.
$$

Remarque 7.36. - Pour le terme $\delta_{n}$, on impose seulement qu'il soit assez proche de 1 . On peut éventuellement prendre $\delta_{n}=1$.

Supposons que $\delta$ est bon pour $\chi$. Pour $i \in \Sigma$, on a : $\operatorname{Re}\left(\chi_{i}\right) \leqslant \operatorname{Re}\left(\chi_{i+1}\right)$, donc $\left|q^{-\chi_{i}+\chi_{i+1}}\right| \geqslant 1$, tandis que si $i \notin \Sigma$, on a $: \operatorname{Re}\left(\chi_{i}\right) \geqslant \operatorname{Re}\left(\chi_{i+1}\right)$, donc $\left|q^{-\chi_{i}+\chi_{i+1}}\right| \leqslant 1$. La somme $(-1)^{\operatorname{card}(\Sigma)+n} \sum_{\boldsymbol{l} \in \mathcal{L}^{0}(\Sigma)} q^{-\langle\chi, \boldsymbol{l}\rangle} x_{1}^{-l_{1}} \cdots x_{n}^{-l_{n}}$ converge alors uniformément sur $\widehat{T}_{u, \delta}$ vers $Q_{\chi}\left(x_{1}, \ldots, x_{n}\right)$, où

$$
Q_{\chi}\left(x_{1}, \ldots, x_{n}\right)=\frac{1}{1-q^{-\chi_{n}} x_{n}} \prod_{i=1}^{n-1} \frac{1}{1-q^{\chi_{i}-\chi_{i+1}} x_{i} x_{i+1}^{-1}}
$$

On obtient :

Proposition 7.37. - Supposons que $\delta$ est bon pour $\chi$. Alors pour tout $f \in \mathcal{H}$, on a l'égalité :

$$
\operatorname{tr}_{\theta-c} \pi[\chi](f)=2 \int_{\widehat{T}_{u, \delta}} p(S(f))\left(x_{1}, \ldots, x_{n}\right) Q_{\chi}\left(x_{1}, \ldots, x_{n}\right) d^{\times} x_{1} \cdots d^{\times} x_{n}
$$

7.4.2. Cas où $\Sigma$ contient $n$. — D'après le corollaire 7.34, l'égalité 7.35 et la formule d'orthogonalité 3.20 , on a :

$$
\begin{aligned}
\operatorname{tr}_{\theta-c} \pi[\chi](f)=(-1)^{\operatorname{card}(\Sigma)-1+n} \int_{\widehat{T}_{u, \delta}} p(S(f))\left(x_{1}, \ldots, x_{n}\right) \\
\cdot\left(2 \sum_{l \in \mathcal{L}^{3}(\Sigma)} q^{-\langle\chi, l\rangle} x_{1}^{-l_{1}} \cdots x_{n}^{-l_{n}}-\sum_{\boldsymbol{l} \in \mathcal{L}^{4}(\Sigma)} q^{-\langle\chi, l\rangle} x_{1}^{-l_{1}} \cdots x_{n}^{-l_{n}}\right) d^{\times} x_{1} \cdots d^{\times} x_{n}
\end{aligned}
$$

pourvu que cela converge. 
Choisissons bon $n$-uplet $\delta=\left(\delta_{1}, \ldots, \delta_{n}\right)$ pour $\chi$. L'expression

$$
(-1)^{\operatorname{card}(\Sigma)-1+n}\left(2 \sum_{\boldsymbol{l} \in \mathcal{L}^{3}(\Sigma)} q^{-\langle\chi, l\rangle} x_{1}^{-l_{1}} \cdots x_{n}^{-l_{n}}-\sum_{\boldsymbol{l} \in \mathcal{L}^{4}(\Sigma)} q^{-\langle\chi, \boldsymbol{l}\rangle} x_{1}^{-l_{1}} \cdots x_{n}^{-l_{n}}\right)
$$

converge uniformément vers $Q_{\chi}^{\prime}\left(x_{1}, \ldots, x_{n}\right)$ sur $\widehat{T}_{u, \delta}$, où

$$
Q_{\chi}^{\prime}\left(x_{1}, \ldots, x_{n}\right)=\frac{1}{1-q^{\chi_{n-1}} x_{n-1}} \frac{1+q^{\chi_{n-1}-\chi_{n}} x_{n-1} x_{n}^{-1}}{1-q^{\chi_{n-1}-\chi_{n}} x_{n-1} x_{n}^{-1}} \prod_{i=1}^{n-2} \frac{1}{1-q^{\chi_{i}-\chi_{i+1}} x_{i} x_{i+1}^{-1}}
$$

On obtient :

$$
\operatorname{tr}_{\theta-c} \pi[\chi](f)=\int_{\widehat{T}_{\delta}} p(S(f))\left(x_{1}, \ldots, x_{n}\right) Q_{\chi}^{\prime}\left(x_{1}, \ldots, x_{n}\right) d^{\times} x_{1} \cdots d^{\times} x_{n}
$$

D'autre part, comme on a déjà dit, la seule condition sur $\delta_{n}$ pour que $\delta$ soit bon pour $\chi$ est que $\delta_{n}$ soit assez proche de 1 . On peut donc choisir $\delta_{n}=1$. Mais si $\delta=\left(\delta_{1}, \ldots, \delta_{n}=1\right)$ est bon pour $\chi$, alors par le changement de variable $x_{n} \mapsto x_{n}^{-1}$, le membre de droite de l'égalité précédente est aussi égal à

$$
\int_{\widehat{T}_{\delta}} p(S(f))\left(x_{1}, \ldots, x_{n}\right) Q_{\chi}^{\prime}\left(x_{1}, \ldots, x_{n}^{-1}\right) d^{\times} x_{1} \cdots d^{\times} x_{n}
$$

Par ailleurs, on a l'égalité suivante, qui découle du fait que $\chi_{n}=\varepsilon$ :

$Q_{\chi}^{\prime}\left(x_{1}, \ldots, x_{n}\right)+Q_{\chi}^{\prime}\left(x_{1}, \ldots, x_{n-1}, x_{n}^{-1}\right)=2\left(Q_{\chi}\left(x_{1}, \ldots, x_{n}\right)+Q_{\chi}\left(x_{1}, \ldots, x_{n-1}, x_{n}^{-1}\right)\right)$

C'est une fonction sans pôles en $x_{n}=1$ tandis que la fonction $Q_{\chi} \mathrm{y}$ en a. Le résultat suivant découle alors de ce qui précède :

Proposition 7.38. - Soit $\chi \in \mathcal{C}(\chi)$, et soit $\delta$ un bon $n$-uplet pour $\chi$. Alors, on a l'égalité :

$$
\begin{aligned}
\operatorname{tr}_{\theta-c} \pi[\chi](f)= & \int_{\widehat{T}_{\delta}} p(S(f))\left(x_{1}, \ldots, x_{n}\right) \\
& \cdot\left(Q_{\chi}\left(x_{1}, \ldots, x_{n}\right)+Q_{\chi}\left(x_{1}, \ldots, x_{n}^{-1}\right)\right) d^{\times} x_{1} \cdots d^{\times} x_{n}
\end{aligned}
$$

Notons que le choix de $\delta$ dépend de $\chi$.

7.4.3. Élimination des pôles. - On va démontrer le résultat principal suivant de ce chapitre. Posons :

$$
Q_{\pi}\left(x_{1}, \ldots, x_{n}\right)=\frac{2}{\left|W\left(C_{n}\right)\right|} \sum_{w \in W\left(C_{n}\right)} \sum_{\chi \in \mathcal{C}(\pi)} Q_{\chi}^{w}\left(x_{1}, \ldots, x_{n}\right)
$$

THÉORÈme 7.39. - La fonction $Q_{\pi}$ n'a pas de pôles au voisinage de $\widehat{T}_{u}$. Pour tout $f \in \mathcal{H}$, on a l'identité :

$$
\operatorname{tr}_{\theta-c} \pi(f)=\int_{\widehat{T}_{u}} p(S(f))\left(x_{1}, \ldots, x_{n}\right) Q_{\pi}\left(x_{1}, \ldots, x_{n}\right) d^{\times} x_{1} \cdots d^{\times} x_{n}
$$

La démonstration de ce résultat se fait par étapes. 
Étape 1. - Pour $\chi \in \mathcal{C}(\pi)$, posons :

$$
\operatorname{Pol}(\chi)=\left\{1 \leqslant i \leqslant n ; \operatorname{Re}\left(\chi_{i}\right)=\operatorname{Re}\left(\chi_{i+1}\right)\right\}
$$

Un raisonnement analogue à celui du lemme 7.21 montre que si $i, j \in \operatorname{Pol}(\chi)$ alors $|i-j| \geqslant 2$. On dira que deux caractères $\chi$ et $\chi^{\prime}$ dans $\mathcal{C}(\pi)$ sont équivalents $\operatorname{si} \operatorname{Pol}(\chi)=$ $\operatorname{Pol}\left(\chi^{\prime}\right)$. Pour $\Xi$ une classe d'équivalence, on définit :

$$
\operatorname{tr}_{\theta-c} \pi[\Xi]=\sum_{\chi \in \Xi} \operatorname{tr}_{\theta-c} \pi[\chi]
$$

de sorte que, pour tout $f \in \mathcal{H}$,

$$
\operatorname{tr}_{\theta-c} \pi(f)=\sum_{\Xi} \operatorname{tr}_{\theta-c} \pi[\Xi](f)
$$

où la somme est portée sur toutes les classes d'équivalence $\Xi$.

Étape 2. - Fixons une classe $\Xi$ et un caractère $\chi_{0} \in \Xi$. Pour simplifier, supposons que $n \notin \operatorname{Pol}\left(\chi_{0}\right)$. Alors les éléments de $\Xi$ sont exactement les caractères de la forme $\left(\prod_{i \in \Delta} s_{i} s_{2 n+1-i}\right) \chi_{0}$, où $\Delta \subset \operatorname{Pol}\left(\chi_{0}\right)$, et $s_{j}$ désigne la symétrie élémentaire qui échange $j$ et $j+1$.

Si $\delta$ est un bon $n$-uplet pour $\chi_{0}$ alors $\left(\prod_{i \in \Delta} s_{i}\right) \delta$ est bon pour $\left(\prod_{i \in \Delta} s_{i} i s_{2 n+1-i}\right) \chi_{0}$. D'après la proposition 7.37 , puis un changement de variables, on a :

$$
\operatorname{tr}_{\theta-c} \pi[\Xi](f)=2 \int_{\widehat{T}_{u, \delta}} p(S(f))\left(x_{1}, \ldots, x_{n}\right) Q_{\Xi}\left(x_{1}, \ldots, x_{n}\right) d^{\times} x_{1} \cdots d^{\times} x_{n}
$$

où

$$
Q_{\Xi}=\sum_{\Delta \subset \operatorname{Pol}\left(\chi_{0}\right)} Q_{\left(\prod_{i \in \Delta} s_{i} s_{2 n+1-i}\right) \chi_{0}}^{\prod_{i \in \Delta} s_{i} s_{2 n+1-i}}
$$

Étape 3. - Montrons que la fonction $Q_{\Xi}$ n'a pas de pôles au voisinage de $T_{u}$. En effet, ses pôles possibles sont les hyperplans $x_{i}= \pm x_{i+1}$, pour $i \in \operatorname{Pol}\left(\chi_{0}\right)$ (pour simplifier, on a supposé $n \notin \operatorname{Pol}\left(\chi_{0}\right)$, rappelons-le). Or, pour $i \in \operatorname{Pol}\left(\chi_{0}\right)$ fixé, on peut décomposer :

$$
Q_{\Xi}=\sum_{\substack{\Delta \subset \operatorname{Pol}\left(\chi_{0}\right) \\ i \notin \Delta}}\left(Q_{\left(\prod_{j \in \Delta} s_{j} s_{2 n+1-j}\right) \chi_{0}}^{\prod_{j \in} s_{2 n+1-j}}+Q_{\left(s_{i} s_{2 n+1-i} \prod_{j \in \Delta} s_{j} s_{2 n+1-j}\right) \chi_{0}}^{s_{i} s_{2 n+1} \prod_{j \in \Delta} s_{j} s_{2 n+1-j}}\right)
$$

Pour $\Delta$ fixé, la fonction $Q_{\left(\prod_{j \in \Delta} s_{j} s_{2 n+1-j}\right) \chi_{0}}^{\prod_{j \in \Delta} s_{j} s_{2 n+1-j}}+Q_{\left(s_{i} s_{2 n+1-i} \prod_{j \in \Delta} s_{j} s_{2 n+1-j}\right) \chi_{0}}^{s_{i} s_{2 n+1-i} \prod_{j \in \Delta} s_{j} s_{2 n+1-j}}$ n'a pas de pôles en $x_{i}= \pm x_{i+1}$. En effet, posons $\chi=\left(\prod_{j \in \Delta} s_{j} s_{2 n+1-j}\right) \chi_{0}$, et écrivons $\chi=\left(\chi_{1}, \ldots, \chi_{2 n+1}\right)$ alors, d'après la définition de $Q_{\chi}$, la somme $Q_{\chi}\left(x_{1}, \ldots, x_{n}\right)+$ $Q_{s_{i} s_{2 n+1-i \chi}}^{s_{i} s_{2 n+1-i}}\left(x_{1}, \ldots, x_{n}\right)$ est produit de

$$
\prod_{\substack{j<i-1, \text { ou } \\ j>i+1}} \frac{1}{1-q^{\chi_{j}-\chi_{j+1}} x_{j} x_{j+1}^{-1}}
$$


et de

$$
\begin{aligned}
& \frac{1}{1-q^{\chi_{i-1}-\chi_{i}} x_{i-1} x_{i}^{-1}} \frac{1}{1-q^{\chi_{i}-\chi_{i+1}} x_{i} x_{i+1}^{-1}} \frac{1}{1-q^{\chi_{i+1}-\chi_{i+2}} x_{i+1} x_{i+2}^{-1}} \\
& +\frac{1}{1-q^{\chi_{i-1}-\chi_{i+1}} x_{i-1} x_{i+1}^{-1}} \frac{1}{1-q^{\chi_{i+1}-\chi_{i}} x_{i+1} x_{i}^{-1}} \frac{1}{1-q^{\chi_{i}-\chi_{i+2}} x_{i} x_{i+2}^{-1}}
\end{aligned}
$$

Le terme 7.41 n'a pas de pôle en $x_{i}= \pm x_{i+1}$. D'autre part, un calcul élémentaire montre que 7.42 est égal à

$$
\begin{aligned}
\left(1-q^{\chi_{i-1}-\chi_{i+2}} x_{i-1} x_{i+2}^{-1}\right) & \frac{1}{1-q^{\chi_{i-1}-\chi_{i}} x_{i-1} x_{i}^{-1}} \frac{1}{1-q^{\chi_{i+1}-\chi_{i+2}} x_{i+1} x_{i+2}^{-1}} \\
& \cdot \frac{1}{1-q^{\chi_{i-1}-\chi_{i+1}} x_{i-1} x_{i+1}^{-1}} \frac{1}{1-q^{\chi_{i}-\chi_{i+2}} x_{i} x_{i+2}^{-1}}
\end{aligned}
$$

qui n'a pas de pôles non plus en $x_{i}= \pm x_{i+1}$.

Étape 4. - On peut donc remplacer $\widehat{T}_{u, \delta}$ par $\widehat{T}_{u}$ dans la formule 7.40. En sommant sur toutes les classes, on obtient :

$$
\operatorname{tr}_{\theta-c} \pi(f)=2 \int_{\widehat{T}_{u, \delta}} p(S(f))\left(x_{1}, \ldots, x_{n}\right) \sum_{\Xi} Q_{\Xi}\left(x_{1}, \ldots, x_{n}\right) d^{\times} x_{1} \cdots d^{\times} x_{n}
$$

Par invariance, on peut alors symétriser cette formule en remplaçant la fonction $\sum_{\Xi} Q_{\Xi} \operatorname{par} \frac{1}{|W|} \sum_{w \in W} \sum_{\Xi} Q_{\Xi}^{w}$. Mais cette fonction multipliée par le facteur 2 figurant dans l'expression ci-dessus est égale à $Q_{\pi}$ par construction des $Q_{\Xi}$, ce qui démontre le théorème. 


\title{
CHAPITRE 8
}

\author{
LE CAS $\pi=\mathrm{St}_{1} \times \xi \mathrm{St}_{2 h+1} \times \xi \mathrm{St}_{2 k+1}$
}

Il s'agit du cas où $\left(\alpha^{+}, \alpha^{-}, \beta\right)=((1),(2 h+1,2 k+1), \varnothing)$, où $h>k \geqslant 0$ deux entiers tels que $h+k+1=n$. Pour $k=0$, on retrouve le cas précédent. On peut donc supposer que $k>0$.

Dans ce cas, le sous-groupe parabolique standard $\mathbf{R}=\mathbf{L V}$ est paramétré par la partition $(1,2 h+1,2 k+1)$. Le groupe de Weyl $W^{L}$ de $L$ est donc isomorphe à $\mathfrak{S}_{1} \times \mathfrak{S}_{2 h+1} \times \mathfrak{S}_{2 k+1}$. Notons, comme précédemment $V$ l'espace de $\pi$ et $A \in \operatorname{End}_{\mathbb{C}}(V)$ l'opérateur d'entrelacement entre $\pi$ et $\pi^{\theta}$ normalisé comme en 2.3. La méthode employée ici est similaire au cas précédent.

\subsection{Le module de Jacquet $\pi_{U}$, où $\mathbf{B}=$ TU}

Dans ce cas le caractère non ramifié $\eta$ de $T$ est donné par la formule :

$$
\eta=(0, h+\varepsilon, \ldots,-h+\varepsilon, k+\varepsilon, \ldots,-k+\varepsilon)
$$

On définit l'ensemble $\mathcal{C}(\pi)$ comme dans le cas précédent. Soit $\chi=\left(\chi_{1}, \ldots, \chi_{2 n+1}\right) \in$ $\mathcal{C}(\pi)$ un caractère $\theta$-invariant de $T$ et soit $w$ un élément de $\left[W / W^{L}\right]$ tel que $\chi=w \eta$. Calculons $\operatorname{tr}_{\theta} \pi_{U}[\chi]$.

8.1.1. Coupures de $\chi$. - On garde la définition de $I(2 n+1)$ du cas précédent : $I(2 n+1)=[1,2 n+1]$. Par contre, on pose $: I(h)=[2,2 h+2]$ et $I(k)=[2 h+3,2 n+1]$. Pour $i \in[h,-h]$, posons $a_{i}=h-i+2$, et pour $i \in[k,-k]$, posons $b_{i}=2 h+3+k-i$. On peut donc écrire $I(h)=\left[a_{h}, a_{-h}\right], I(k)=\left[b_{k}, b_{-k}\right]$. Posons, pour $i=h, \ldots,-h$, $E_{i}=\left\{j ; \chi_{j}=i+\varepsilon\right\}$. L'ensemble $E_{i}$ a un élément si $h \geqslant|i|>k$, et deux éléments si $|i| \leqslant k$. Posons alors :

$$
E(i)= \begin{cases}\{e(i)\} & \text { pour } h \geqslant|i|>k \\ \left\{e^{\prime}(i)<e^{\prime \prime}(i)\right\} & \text { pour } k \geqslant|i|\end{cases}
$$


On a donc :

$$
\begin{aligned}
e(i) & =w\left(a_{i}\right) \quad \text { si }|i|>k \\
\left\{e^{\prime}(i),\left(e^{\prime \prime}(i)\right)\right\} & =\left\{w\left(a_{i}\right), w\left(b_{i}\right)\right\} \quad \text { si }|i| \leqslant k
\end{aligned}
$$

Supposons $i \in[k,-k+1]$. Puisque $w \in\left[W / W^{L}\right]$, il est croissant sur chacun des intervalles $I(h)$ et $I(k)$, donc $w\left(a_{i}\right)<w\left(a_{i-1}\right), w\left(b_{i}\right)<w\left(b_{i-1}\right)$. On a donc l'une des deux possibilités suivantes :

i) Soit $e^{\prime}(i)<e^{\prime \prime}(i)<e^{\prime}(i-1)<e^{\prime \prime}(i-1)$;

ii) Soit $e^{\prime}(i)<e^{\prime}(i-1)<e^{\prime \prime}(i)<e^{\prime \prime}(i-1)$.

Pour $i=k+1$, de même, on a l'une des deux possibilités :

i) Soit $e(k+1)<e^{\prime}(k)<e^{\prime \prime}(k)$;

ii) Soit $e^{\prime}(k)<e(k+1)<e^{\prime \prime}(k)$.

On a les inégalités analogues pour $i=-k$.

On dira qu'il y a coupure entre $i$ et $i-1$ lorsque $i$ ) se produit. S'il y a coupure entre $k+1$ et $k$ on dit aussi qu'il y a coupure entre $-k$ et $-k-1$. L'ensemble des coupures de $\chi$ que l'on va noter $\operatorname{par} \operatorname{Coup}(\chi)$ est alors symétrique par rapport à 0 .

Lemme 8.1. - L'ensemble $\operatorname{Coup}(\chi)$ n'est pas vide.

Démonstration. - On le montre par récurrence sur $n$, en acceptant le cas $k=0$. Si $k=0$, il est facile de voir que $(1,0)$ et $(0,-1)$ sont les coupures de $\chi$. Si $k>0$, comme $\chi=w \eta$ avec $w$ croissant sur $I(h)$ et $I(k)$, on a l'une des deux possibilités :

i) Soit $\chi_{1}=h+\varepsilon, \chi_{2 n+1}=-h+\varepsilon$, en d'autres termes $w\left(a_{h}\right)=1, w\left(a_{-h}\right)=2 n+1$;

ii) Soit $\chi_{1}=k+\varepsilon, \chi_{2 n+1}=-k+\varepsilon$, en d'autres termes $w\left(b_{k}\right)=1, w\left(b_{-k}\right)=2 n+1$.

Si $i i)$ se produit on conclut par l'hypothèse de récurrence pour $n^{\prime}=n-1, h^{\prime}=h$, $k^{\prime}=k-1, \chi^{\prime}=\left(\chi_{2}, \ldots, \chi_{2 n}\right)$. Si $\left.i\right)$ se produit et $h-1>k$ on conclut par l'hypothèse de récurrence pour $n^{\prime}=n-1, h^{\prime}=h-1, k^{\prime}=k, \chi^{\prime}=\left(\chi_{2}, \ldots, \chi_{2 n}\right)$. Enfin, si $\left.i\right)$ se produit et $h-1=k$, alors $(h, h-1=k)$ est une coupure de $\chi$.

\subsubsection{Propriétés des coupures}

Lemme 8.2. - Soit $(i, i-1)$ une coupure de $\chi$, alors :

$$
e^{\prime}(i-1)=e^{\prime \prime}(i)+1
$$

Démonstration. - On a $w\left(a_{i}\right)=e^{\prime}(i)$ ou $e^{\prime \prime}(i)$. Puisque $e^{\prime}(i)<e^{\prime \prime}(i)$, on a donc $w\left(a_{i}\right) \leqslant e^{\prime \prime}(i)$. Or, $w$ est croissant sur $I(h)$ et $I(k)$, on obtient;

$$
\left.\begin{array}{l}
w\left(\left[a_{h}, a_{i}\right]\right) \subset\left[1, e^{\prime \prime}(i)\right] \\
w\left(\left[b_{k}, b_{i}\right]\right) \subset\left[1, e^{\prime \prime}(i)\right]
\end{array}\right\}(*)
$$

Par un raisonnement similaire, on a aussi :

$$
\left.\begin{array}{l}
w\left(\left[a_{i-1}, a_{-h}\right]\right) \subset\left[e^{\prime}(i-1), 2 n+1\right] \\
w\left(\left[b_{i-1}, b_{-k}\right]\right) \subset\left[e^{\prime}(i-1), 2 n+1\right]
\end{array}\right\}(* *)
$$


Par suite, on a l'une des deux possibilités :

(1) Soit $e^{\prime \prime}(i)+1=e^{\prime}(i-1)$, c'est-à-dire l'égalité cherchée;

(2) Soit $e^{\prime \prime}(i)+2=e^{\prime}(i-1)$ et $e^{\prime \prime}(i)+1=w(1)=n+1$. On a donc $\chi_{n}=i+\varepsilon$ et $\chi_{n+2}=i-1+\varepsilon$. Par symétrie ( $\chi$ est $\theta$-stable), on doit avoir $i+i-1=0$, ce qui est impossible.

Grâce à ce résultat, on dira aussi qu'il y a coupure entre $\chi_{e^{\prime \prime}(i)}$ et $\chi_{e^{\prime \prime}(i)+1}$. On munit l'ensemble $\operatorname{Coup}(\chi)$ de l' ordre déduit de celui sur $\mathbb{Z}$.

Lemme 8.3. - L'application

$$
\begin{aligned}
& \operatorname{Coup}(\chi) \longrightarrow \mathbb{Z} \\
& (i, i-1) \longmapsto e^{\prime}(i-1)
\end{aligned}
$$

est décroissante.

Démonstration. - En effet, pour $j<i$, on a : $e^{\prime}(j-1)=w\left(a_{j-1}\right)$ ou $w\left(b_{j-1}\right)$, et on applique $(* *)$ ci-dessus.

Soient $(i, i-1)$ et $(j, j-1)$ deux coupures successives de $\chi$ avec $i>j$.

Lemme 8.4. - La famille des $\chi_{l}$ pour $l \in\left[e^{\prime}(i-1), e^{\prime \prime}(j)\right]$ est égale, à l'ordre près, à $\{i-1+\varepsilon, i-1+\varepsilon, i-2+\varepsilon, i-2+\varepsilon, \ldots, j+\varepsilon, j+\varepsilon\}$ éventuellement augmenté de 0.

Démonstration. - Il résulte de $(*)$ et $(* *)$ ci-dessus que $\left[e^{\prime}(i-1), e^{\prime \prime}(j)\right]$ est l'image par $w$ de $\left[a_{i-1}, a_{j}\right] \cup\left[b_{i-1}, b_{j}\right]$ éventuellement augmenté de 1 , d'où le lemme.

Appelons coupure dominante la plus grande coupure de $\chi$. Soit $\left(i_{\max }, i_{\max }-1\right)$ la coupure dominante de $\chi$. Alors, a aussi : $\left[1, e^{\prime \prime}\left(i_{\max }\right)\right]=w\left(\left[a_{h}, a_{i_{\max }}\right]\right) \cup w\left(\left[b_{k}, b_{i_{\max }}\right]\right)$.

Lemme 8.5. - On a l'égalité $: \operatorname{dim} V_{U}[\chi]=2^{\operatorname{card}(\operatorname{Coup}(\chi))-1}$

Démonstration. - Par application de la filtration de Bernstein-Zelevinsky, la dimension de $V_{U}[\chi]$ est égale au nombre des $w \in\left[W / W^{L}\right]$ tels que $w \eta=\chi$. Soit $w$ un tel élément. Identifions $W$ à l'ensemble des permutations de $I(2 n+1)=\{1\} \cup I(h) \cup I(k)$. Le terme $\eta_{1}$ est l'unique terme égal à 0 parmi les $\eta_{i}$, donc $w(1)=n+1$. De même, par unicité, pour $i \in[h, k+1] \cup[-k+1,-h]$, on a $w\left(a_{i}\right)=e_{i}$. Soit donc $i \in[k+1,-k]$. Si $(i, i-1)$ est une coupure de $\chi$ qui n'est pas minimale, supposons $w\left(a_{i}\right)$ et $w\left(b_{i}\right)$ donnés, alors on a deux possibilités pour $w\left(a_{i-1}\right)$ et $w\left(b_{i-1}\right)$ :

(1) $w\left(a_{i-1}\right)=e^{\prime}(i-1), w\left(b_{i-1}\right)=e^{\prime \prime}(i-1)$;

(2) $w\left(a_{i-1}\right)=e^{\prime \prime}(i-1), w\left(b_{i-1}\right)=e^{\prime}(i-1)$.

S'il n'y a pas de coupure entre $i$ et $i-1$ (ou $i=-k$ ), alors étant donnés $w\left(a_{i}\right)$ et $w\left(b_{i}\right)$, les éléments $w\left(a_{i-1}\right)$ et $w\left(b_{i-1}\right)$ sont complètement fixés. En effet, puisque $(i, i-1)$ n'est pas une coupure pour $\chi$. on a

$$
e^{\prime}(i)<e^{\prime}(i-1)<e^{\prime \prime}(i)<e^{\prime \prime}(i-1)
$$


Par hypothèse, $w \in\left[W / W^{L}\right]$, il est croissant sur chacun des intervalles $I(h)$ et $I(k)$. Ainsi, si $w\left(a_{i}\right)=e^{\prime}(i), w\left(b_{i}\right)=e^{\prime \prime}(i)$ alors $w\left(b_{i-1}\right)$ qui est est plus grand que $e^{\prime \prime}(i)$ doit forcément être égal à $e^{\prime \prime}(i-1)$, du coup $w\left(a_{i-1}\right)=e^{\prime}(i-1)$. Le même raisonnement montre également que si $\left.w\left(a_{i}\right)=e^{\prime \prime}(i), w\left(b_{i}\right)=e^{\prime}(i)\right)$ alors $w\left(a_{i-1}\right)=e^{\prime \prime}(i-1), w\left(b_{i-1}\right)=e^{\prime}(i-1)$. Par contre, pour chaque coupure non minimale de $\chi$, on a deux possibilités pour le couple $w\left(a_{i-1}\right), w\left(b_{i-1}\right)$. Le nombre des $w \in\left[W / W^{L}\right]$ tels que $w \eta=\chi$ est donc égal à $2^{\text {card }(\operatorname{Coup}(\chi))-1}$, ce qu'il fallait démontrer.

8.1.3. Construction de $\varrho$ - - Soit $\left(i_{\max }, i_{\max }-1\right)$ la coupure dominante de $\chi$. Posons :

$$
d=n-e^{\prime \prime}\left(i_{\max }\right)
$$

D'après la remarque qui suit le lemme 8.4 , on a aussi $d=2 i_{\max }-1$.

Soit $\mathbf{Q}=\mathbf{M}_{\mathbf{Q}} \mathbf{N}_{\mathbf{Q}}$ le sous-groupe parabolique standard $\theta$-stable associé à la partition $(n-d, 2 d+1, n-d)$. Pour $a \geqslant b$ deux entiers, on note $\mathrm{St}_{a, b}$ la représentation $|\cdot|^{\frac{a+b}{2}} \mathrm{St}_{a-b+1}$. Posons :

$$
\varrho=\left(\xi \mathrm{St}_{h, i_{\max }} \times \xi \mathrm{St}_{k, i_{\max }}\right) \otimes\left(\xi \mathrm{St}_{d} \times \mathrm{St}_{1} \times \xi \mathrm{St}_{d}\right) \otimes\left(\xi \mathrm{St}_{-i_{\max },-k} \times \xi \mathrm{St}_{-i_{\max },-h}\right)
$$

C'est une représentation lisse, irréductible, $\theta$-stable de $M_{Q}$. Notons $E$ son espace.

8.1.4. Action de $A$ sur $V_{U}[\chi]$. — La proposition 7.3, dont on rappelle ci-dessus l'énoncé, reste valable.

\section{Proposition 8.6}

(1) La représentation @ s'identifie à une sous-représentation de $\pi_{N_{Q}}$.

(2) L'opérateur A conserve $E$ et réalise un opérateur d'entrelacement entre $\varrho$ et $\varrho^{\theta}$.

(3) Avec les notations usuelles, l'espace $E_{U}$ contient $V_{U}[\chi]$, en fait on a l'égalité :

$$
E_{U}[\chi]=V_{U}[\chi]
$$

Démonstration. - Soit $w_{1}$ l'unique élément de $W=\mathfrak{S}_{2 n+1}$ qui envoie de façon croissante

-1 sur $n-d+1$;

$-\left[a_{h}, a_{i_{\max }}\right]$ sur $\left[1, h-i_{\max }+1\right]$;

$-\left[b_{k}, b_{i_{\max }}\right]$ sur $\left[h-i_{\max }+2, n-d\right]$;

$-\left[a_{-i_{\max }}, a_{-h}\right]$ sur $\left[n+d+2, n+d+2+h-i_{\max }\right]$;

$-\left[b_{-i_{\max }}, b_{-k}\right]$ sur $\left[n+d+3+h-i_{\max }, 2 n+1\right]$;

$-\left[a_{i_{\max }-1}, a_{1-i_{\max }}\right]$ sur $[n-d+2, n+1]$;

- $\left[b_{i_{\max }-1}, b_{1-i_{\max }}\right]$ sur $[n+2, n+d+1]$.

On vérifie que $w_{1} \in\left[W^{M_{Q}} \backslash W / W^{L}\right]$, et la composante $\mathcal{F}_{w_{1}}(\pi)$ dans la filtration de Bernstein-Zelevinsky de $\pi_{N_{Q}}$ est isomorphe à $\varrho$ (notons que $\mathrm{St}_{1} \times \xi \mathrm{St}_{d} \times \xi \mathrm{St}_{d}$ est isomorphe à $\xi \mathrm{St}_{d} \times \mathrm{St}_{1} \times \xi \mathrm{St}_{d}$ et $\xi \mathrm{St}_{-i_{\max },-k} \times \xi \mathrm{St}_{-i_{\max },-h}$ est isomorphe à $\left.\xi \mathrm{St}_{-i_{\max },-h} \times \xi \mathrm{St}_{-i_{\max },-k}\right)$. En plus, on montre que c'est l'unique composante sur 
laquelle le centre $Z$ de $M$, qui s'identifie de façon naturelle à $\left(F^{\times}\right)^{3}$, agit par le caractère non ramifié $\left(h+\cdots+i_{\max }+k+\cdots+i_{\max }, 0,-i_{\max }-\cdots-h-i_{\max }-\cdots-k\right)$. Ce dernier est $\theta$-stable, on en conclut que l'espace $E$ de $\rho$ est sous-espace de $V_{N}$, en facteur direct et qu'il est stable sous l'action de l'opérateur d'entrelacement $A$. Ce qui démontre les deux premiers points. Pour démontrer le dernier point, il suffit de montrer l'égalité des dimensions. La dimension de $V_{U}[\chi]$ est égale à $2^{\operatorname{card}(\operatorname{Coup}(\chi))-1}$ d'après le lemme 8.5. D'autre part, par la même méthode, on montre que la dimension de $E_{U}[\chi]$ est donnée par la même formule, d'où le résultat.

Posons :

$$
\tau=\left(\xi \mathrm{St}_{h, i_{\max }} \otimes \xi \mathrm{St}_{k, i_{\max }}\right) \otimes\left(\xi \mathrm{St}_{d} \otimes \mathrm{St}_{1} \otimes \xi \mathrm{St}_{d}\right) \otimes\left(\xi \mathrm{St}_{-i_{\max },-k} \otimes \xi \mathrm{St}_{-i_{\max },-h}\right)
$$

Par définition, $\varrho$ est l'induite parabolique de $\tau$. Posons :

$$
\begin{gathered}
\chi_{0}=\left(h+\varepsilon, \ldots, i_{\max }+\varepsilon, k+\varepsilon, \ldots, i_{\max }+\varepsilon, i_{\max }-1+\varepsilon, \ldots,-i_{\max }+1+\varepsilon, 0,\right. \\
\left.i_{\max }-1+\varepsilon, \ldots,-i_{\max }+1+\varepsilon,-i_{\max }+\varepsilon, \ldots,-k+\varepsilon,-i_{\max }+\varepsilon, \ldots,-h+\varepsilon\right)
\end{gathered}
$$

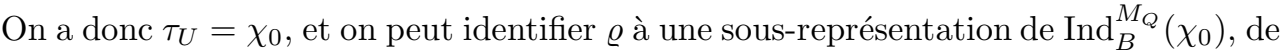
sorte que l'application $f \mapsto f^{\theta}$ soit bien définie et réalise un opérateur d'entrelacement entre $\varrho$ et $\varrho^{\theta}$.

Lemme 8.7. - Pour tout $f \in E$, on a l'égalité :

$$
A(f)=f^{\theta}
$$

Démonstration. — Identique à celle du lemme 7.4.

8.1.5. Formule pour $\operatorname{tr}_{\theta} \pi_{U}[\chi]$. - Il s'agit de la formule de la proposition 7.5 dont on rappelle ci-dessous l'énoncé. Soit $\mathbf{P}=\mathbf{M}_{\mathbf{P}} \mathbf{N}_{\mathbf{P}}$ le sous-groupe parabolique standard $\theta$-stable paramétré par la partition

$$
\left(h-i_{\max }+1, k-i_{\max }+1, d, 1, d, k-i_{\max }+1, h-i_{\max }+1\right)
$$

Proposition 8.8. - Soit $f$ une fonction localement constante, à support compact sur T. On a l'identité :

$$
\operatorname{tr}_{\theta} \pi_{U}[\chi](f)=\operatorname{card}\left\{w \in\left[W^{M_{Q}} / W^{M_{P}}\right] ; w \chi_{0}=\chi, \theta(w)=w\right\} \int_{T} \chi(t) f(t) d t
$$

Démonstration. — Identique à celle de la proposition 7.5.

Dans le cas présent, on a la formule suivante, qui calcule le terme constant de la formule ci-dessus :

LEMME 8.9. - On a l'égalité :

$$
\operatorname{card}\left\{w \in\left[W^{M_{Q}} / W^{M_{P}}\right], w \chi_{0}=\chi, \theta(w)=w\right\}=2^{\frac{\operatorname{card}(\operatorname{Coup}(\chi))}{2}}
$$


Démonstration. - On va imiter la démonstration du lemme 8.5. Remarquons que la condition $\theta(w)=w$ est équivalente à

$$
w(i)+w(2 n+2-i)=2 n+2
$$

pour tout $i \in I(2 n+1)$.

La partition

$$
\left(h-i_{\max }+1, k-i_{\max }+1, d, 1, d, k-i_{\max }+1, h-i_{\max }+1\right)
$$

de $2 n+1$ correspond de façon évidente à une partition de $I(2 n+1)$, disons $I(2 n+1)=$ $I_{-1,-2} \cup I_{-1,-1} \cup I_{0,-1} \cup I_{0,0} \cup I_{0,1} \cup I_{1,1} \cup I_{1,2}$. Écrivons :

$$
\begin{array}{rlrl}
I_{-1,-2} & =\left[a_{h}, a_{i_{\max }}\right], & & I_{0,0}=\{n+1\}, I_{0,1}=\left[a_{i_{\max }-1}, a_{-i_{\max }+1}\right], \\
I_{-1,-1} & =\left[b_{k}, b_{i_{\max }}\right], & I_{1,1}=\left[b_{-i_{\max }}, b_{-k}\right], I_{1,2}=\left[a_{-i_{\max }}, a_{-h}\right] \\
I_{0,-1} & =\left[a_{i_{\max }-1}, a_{-i_{\max }+1}\right], & &
\end{array}
$$

On a donc $: \chi_{0}=\left(\chi_{0,1}, \ldots, \chi_{0,2 n+1}\right)$ avec $\chi_{0, a_{i}}=a_{i}+\varepsilon, \chi_{0, b_{i}}=b_{i}+\varepsilon, \chi_{0, n+1}=0$.

Soit $w \in\left[W^{M_{Q}} / W^{M_{P}}\right]$ tel que $w \chi_{0}=\chi$. Par définition, $w$ est croissant sur les intervalles $I_{-1,-2}, I_{-1,-1}, I_{0,-1}, I_{0,0}, I_{0,1}, I_{1,1}, I_{1,2}$. Comme $\chi_{0, n+1}=\chi_{n+1}=0$, par unicité, on a $w(n+1)=n+1$. Par unicité, les $w\left(a_{h}\right)$ et $w\left(a_{-h}\right)$ sont uniquement déterminés. Par symétrie, la relation 8.10 est automatiquement vérifiée pour $a_{h}$. Soit $i \in\left[h, i_{\max }+1\right]$. Sachant les valeurs $w\left(a_{i}\right), w\left(b_{i}\right), w\left(a_{-i}\right), w\left(b_{-i}\right)$, et supposons que la relation 8.10 est vérifiée pour $a_{i}, b_{i}$, montrons que les $w\left(a_{i-1}\right), w\left(b_{i-1}\right), w\left(a_{-i+1}\right)$, $w\left(b_{-i+1}\right)$ sont uniquement déterminés et que la relation 8.10 est automatiquement vérifié pour $a_{i-1}, b_{i-1}$. Comme $(i, i-1),(-i+1,-i)$ ne sont pas des coupures, on a :

$$
e^{\prime}(i)<e^{\prime}(i-1)<e^{\prime \prime}(i)<e^{\prime \prime}(i-1), e^{\prime}(-i+1)<e^{\prime}(-i)<e^{\prime \prime}(-i+1)<e^{\prime \prime}(-i)
$$

Supposons que $w\left(a_{i}\right)=e^{\prime}(i), w\left(b_{i}\right)=e^{\prime \prime}(i)$. Alors, par la propriété de croissance de $w$, on a $w\left(a_{i-1}\right)=e^{\prime}(i-1), w\left(b_{i-1}\right)=e^{\prime \prime}(i-1)$. On a supposé que la relation 8.10 est vérifiée pour $a_{i}, b_{i}$. Cette relation, jointe avec la symétrie de $\chi$, donne $: e^{\prime}(i)+e^{\prime \prime}(-i)=$ $e^{\prime \prime}(i)+e^{\prime}(-i)=2 n+2$, donc $w\left(a_{-i}\right)=e^{\prime \prime}(-i), w\left(b_{-i}\right)=e^{\prime}(-i)$. On en déduit, toujours par la propriété de croissance de $w$ que $w\left(b_{-i+1}\right)=e^{\prime}(-i+1), w\left(a_{-i+1}\right)=e^{\prime \prime}(-i+1)$. La symétrie de $\chi$ montre que la relation 8.10 est alors automatiquement vérifiée pour $a_{i-1}, b_{i-1}$. Le cas $w\left(a_{i}\right)=e^{\prime \prime}(i), w\left(b_{i}\right)=e^{\prime}(i)$ se démontre de façon similaire.

Pour $i=i_{\max }-1$, on a deux possibilités pour le couple $w\left(a_{i_{\max }-1}\right), w\left(b_{i_{\max }-1}\right)$. Par contre, par la symétrie de $\chi$, on a une seule possibilité pour le couple $w\left(a_{-i_{\max }+1}\right), w\left(b_{-i_{\max }+1}\right)$ sachant $w\left(a_{i_{\max }-1}\right), w\left(b_{i_{\max }-1}\right)$. On vérifie que la relation 8.10 est alors automatiquement vérifiée pour $w\left(a_{i_{\max }-1}\right), w\left(b_{i_{\max }-1}\right)$.

Plus généralement, par des arguments similaires à ceux de la démonstration du lemme 8.5, pour $i$ positif, sachant les valeurs $w\left(a_{i}\right), w\left(b_{i}\right)$, il y a deux possibilités pour le couple $w\left(a_{i-1}\right), w\left(b_{i-1}\right)$ si $(i, i-1)$ est une coupure et une seule possibilité si $(i, i-1)$ n'est pas une coupure. Mais, par les raisonnements similaires à ceux plus haut, sachant $w\left(a_{i}\right), w\left(b_{i}\right)$ et supposons que la relation 8.10 est vérifiée pour $a_{i}, b_{i}$ alors il y a une 
seule possibilité pour le couple $w\left(a_{-i+1}\right), w\left(b_{-i+1}\right)$ qui satisfait la relation 8.10 pour $a(i-1), b(i-1)$ que $(i, i-1)$ soit une coupure ou non.

Donc, le nombre de tels $w$ est égal à 2 à la puissance le nombre de coupures positives. D'où le résultat.

Corollaire 8.11. - Soit $f$ une fonction lisse, à support compact sur le tore diagonal $T$ de $G$. On a alors l'identité :

$$
\operatorname{tr}_{\theta} \pi_{U}[\chi](f)=2^{\frac{\operatorname{card}(\operatorname{Coup}(\chi))}{2}} \int_{T} \chi(t) f(t) d t
$$

D'où

$$
\operatorname{tr}_{\theta} \pi_{U}(f)=\sum_{\chi \in \mathcal{C}(\pi)} 2^{\frac{\operatorname{card}(\operatorname{Coup}(\chi))}{2}} \int_{T} \chi(t) f(t) d t
$$

\subsection{Les modules de Jacquet $\pi_{N}$ où $\mathbf{P}=\mathbf{M N} \in \mathcal{P}^{\theta}$}

8.2.1. Généralités. - Gardons les notations des paragraphes précédents. Fixons donc un caractère $\theta$-invariant de $A_{M}$, le centre de $M$, de la forme $\bar{\chi}=\operatorname{res}_{P}(w \eta), w \in$ $\left[W^{M} \backslash W / W^{L}\right]$. On peut toujours supposer que la partition $\alpha=\left(\alpha_{1}, \ldots, \alpha_{2 r-1}\right)$ est telle que $\alpha_{i} \leqslant 2$ pour $i \neq r$ et $\alpha_{r} \leqslant 3$. Notons que $\operatorname{res}_{P}(w \eta)$ est $\theta$-invariant veut dire que pour $i=1, \ldots, r$, on a :

$$
\sum_{j \in J\left(\alpha_{i}\right)}(w \eta)_{i}=-\sum_{j \in J\left(\alpha_{2 r-i}\right)}(w \eta)_{j}
$$

En fait, on a le résultat suivant, qui précise cette égalité :

Lemme 8.13. - Pour $i=1, \ldots, r$ on a l'égalité entre deux familles:

$$
\left\{(w \eta)_{i} ; j \in J\left(\alpha_{i}\right)\right\}=\left\{-(w \eta)_{j} ; j \in J\left(\alpha_{2 r-i}\right)\right\}
$$

Démonstration. - Soit $i \in[1, r-1]$. On suppose l'égalité 8.14 prouvée pour tout $i^{\prime}<i$. Si $\alpha_{i}=1$, le lemme résulte de l'égalité 8.12. Si $\alpha_{i}=2$, écrivons : $J\left(\alpha_{i}\right)=$ $\left\{w\left(a_{j}\right), w\left(b_{j^{\prime}}\right)\right\}, J\left(\alpha_{2 r-i}\right)=\left\{w\left(a_{l}\right), w\left(b_{l^{\prime}}\right)\right\}$. Comme $w$ est croissant sur $I(h)$ et $I(k)$, les ensembles $w\left(\left[a_{h}, a_{j+1}\right]\right)$ et $w\left(\left[b_{k}, b_{j+1}\right]\right)$ sont majorés respectivement par $w\left(a_{j}\right)$ et $w\left(b_{j^{\prime}}\right)$, donc $w\left(\left[a_{h}, a_{j+1}\right]\right) \cup w\left(\left[b_{k}, b_{j^{\prime}+1}\right]\right) \subset J\left(\alpha_{1}\right) \cup \cdots \cup J\left(\alpha_{i-1}\right)$. Toujours par la propriété de croissance, on a $w\left(\left[a_{j}, a_{-h}\right]\right) \cup w\left(\left[b_{j}^{\prime}, b_{-k}\right]\right) \subset J\left(\alpha_{i}\right) \cup \cdots \cup J_{2 r-1}$. Enfin, remarquons que $w(1) \in J\left(\alpha_{r}\right)$, on obtient :

$$
w\left(\left[a_{h}, a_{j+1}\right]\right) \cup w\left(\left[b_{k}, b_{j^{\prime}+1}\right]\right)=J\left(\alpha_{1}\right) \cup \cdots \cup J\left(\alpha_{i-1}\right)
$$

Par un raisonnement similaire, on a aussi :

$$
w\left(\left[a_{l-1}, a_{-h}\right]\right) \cup w\left(\left[b_{l^{\prime}-1}, b_{-k}\right]\right)=J\left(\alpha_{2 r-i+1}\right) \cup \cdots \cup J\left(\alpha_{2 r-1}\right)
$$

Appliquons l'hypothèse de récurrence aux deux égalités ci-dessus, on obtient :

$$
[h, j+1] \cup\left[k, j^{\prime}+1\right]=[h,-l+1] \cup\left[k,-l^{\prime}+1\right]
$$

D'où : soit $j=-l, j^{\prime}=-l^{\prime}$ soit $j=-l^{\prime}, j^{\prime}=-l$, ce qui démontre l'égalité 8.14 pour $i$. 
Pour $i=r$, la symétrie résulte de : la famille $(w \eta)_{j}$ est stable par $\theta$; par ce qui précède la famille $\left\{(w \eta)_{j} \mid j \notin J\left(\alpha_{r}\right)\right\}$ l'est. Donc par différence, la famille $\left\{(w \eta)_{j} \mid j \in J\left(\alpha_{r}\right)\right\}$ l'est aussi.

\section{Corollaire 8.15}

(1) Il existe un caractère $\chi \in \mathcal{C}(\pi)$ de restriction $\bar{\chi}$.

(2) Un tel caractère est conjugué par un élément de $W^{M}$ au caractère wn pour un $w \in\left[W^{M} \backslash W / W^{L}\right]^{0}$ tel que $\bar{\chi}=\operatorname{res}_{P}(w \eta)$.

REMARque 8.16. - L'intérêt est que $\chi$ est maintenant $\theta$-invariant alors que $w \eta$ ne l'est pas forcément.

8.2.2. $P$-coupures. - Soit $\chi \in \mathcal{C}(\pi)$ tel que $\operatorname{res}_{P}(\chi)=\bar{\chi}$. Soit $(i, i-1)$ une coupure de $\chi$ avec $i \geqslant 1$. On dira que $(i, i-1)$ est une $P$-coupure de $\bar{\chi}$ si les conditions suivantes sont satisfaites :

- $\left\{e^{\prime \prime}(i), e^{\prime}(i-1)\right\}$ n'est pas de la forme $J\left(\alpha_{l}\right), l=1, \ldots, 2 r-1$;

- $\left\{e^{\prime}(i-1), e^{\prime \prime}(i-1)\right\}$ n'est pas de la forme $J\left(\alpha_{l}\right), l=1, \ldots, 2 r-1$;

- $\left\{e^{\prime}(i-1), e^{\prime \prime}(i-1), n+1\right\}$ n'est pas de la forme $J\left(\alpha_{r}\right)$.

Par symétrie, si $(i, i-1)$ est une $P$-coupure de $\bar{\chi}$ avec $i \geqslant 1$, on dira aussi que $(-i+1,-i)$ est une $P$-coupure de $\bar{\chi}$. Notons $\operatorname{Coup}_{P}(\bar{\chi})$ l'ensemble de telles coupures. Cette définition est indépendante du choix de $\chi$.

Remarque 8.17. - Il se peut que l'ensemble $\operatorname{Coup}_{P}(\bar{\chi})$ soit vide si $\mathbf{P} \neq \mathbf{B}$.

\subsubsection{Formule pour $\operatorname{tr}_{\theta} \pi_{N}[\bar{\chi}]$ lorsque $\operatorname{Coup}_{P}(\bar{\chi}) \neq \varnothing$}

8.2.3.1. Construction de @ lorsque $\operatorname{Coup}_{P}(\bar{\chi}) \neq \varnothing$. $-\operatorname{Si} \operatorname{Coup}_{P}(\bar{\chi}) \neq \varnothing$, on définit, comme dans le cas $\mathbf{P}=\mathbf{B}$, la $P$-coupure dominante comme la plus grande des $P$ - coupures de $\bar{\chi}$. Notons-la $i_{P, \max }$. La construction de $\varrho$ est alors identique au cas $\mathbf{P}=\mathbf{B}$ : il suffit de changer $i_{\max }$ en $i_{P, \max }$. Les propriétés de $\varrho$ restent valables. Rappelons que $\varrho$ est une représentation du facteur de Lévi d'un sous-groupe parabolique $\mathbf{Q}$ contenant $\mathbf{P}$. Comme dans le premier cas, on obtient alors le résultat suivant :

Proposition 8.18. - Soit $f$ un élément de $\mathcal{H}(M)$, on a l'égalité :

$\operatorname{tr}_{\theta} \pi_{N}[\bar{\chi}](f)=\operatorname{card}\left\{w \in\left[W^{M} \backslash W^{M_{Q}} / W^{L}\right]^{0} ; \operatorname{res}_{P}(w \eta)=\bar{\chi}, \theta(w)=w\right\} \operatorname{tr} \operatorname{Ind}_{B}^{M}(\chi)(f)$

On a un résultat analogue à celui du cas $\mathbf{P}=\mathbf{B}$, qui donne une formule pour le terme constant dans la formule ci-dessus :

Lemme 8.19. - On a l'égalité :

$$
\operatorname{card}\left\{w \in\left[W^{M} \backslash W^{M_{Q}} / W^{L}\right]^{0} ; \overline{w \eta}=\bar{\chi}, \theta(w)=w\right\}=2^{\frac{\operatorname{card}\left(\operatorname{Coup}_{P}(\bar{x})\right)}{2}}
$$


8.2.3.2. Cas $\operatorname{Coup}_{P}(\bar{\chi})=\varnothing$.- Dans ce cas, l'espace $V_{N}^{K_{M}}[\bar{\chi}]$ est de dimension 1. On montre de façon usuelle que l'opérateur $A$ agit trivialement sur ce dernier. Par suite,

$$
\operatorname{tr}_{\theta} \pi_{N}[\bar{\chi}](f)=\operatorname{tr} \operatorname{Ind}_{B}^{M}(\chi)(f)
$$

Nous avons ainsi démontré le résultat suivant qui est valable dans les deux cas :

Corollaire 8.20. - On a l'égalité :

$$
\operatorname{tr}_{\theta} \pi_{N}[\bar{\chi}](f)=2^{\frac{\operatorname{card}\left(\operatorname{Coup}_{P}(\bar{\chi})\right)}{2}} \operatorname{tr} \operatorname{Ind}_{B}^{M}(\chi)(f)
$$

8.2.4. Formule pour $\operatorname{tr}_{\theta-c} \pi$. - On peut résumer les résultats obtenus sous la forme :

Proposition 8.21. - Pour $\mathbf{P}=\mathbf{M N} \in \mathcal{P}^{\theta-s t}, \bar{\chi} \in \mathcal{C}_{P}(\pi)$, choisissons un $\chi \in \mathcal{C}(\pi)$ de restriction $\bar{\chi}$. Alors pour tout $f \in \mathcal{H}(G)$, on a l'égalité :

$$
\operatorname{tr}_{\theta-c} \pi(f)=\sum_{\mathbf{P}=\mathbf{M N} \in \mathcal{P}^{\theta-s t}} \sum_{\bar{\chi} \in \mathcal{C}(\pi)}(-1)^{a_{P}^{\theta}-a_{G}^{\theta} 2^{\frac{\operatorname{card}\left(\operatorname{Coup}_{P}(\bar{\chi})\right.}{2}}} \operatorname{tr} \operatorname{Ind}_{B}^{M}(\chi)\left(\widehat{\chi}_{N}^{\theta} f^{P}\right)
$$

Nous nous inspirons du premier cas pour récrire la formule de la proposition précédente. Soit $\bar{\chi} \in \mathcal{C}_{P}(\pi)$. Il existe un unique caractère $\chi \in \mathcal{C}(\pi)$ avec les propriétés suivantes :

$-\operatorname{res}_{P}(\chi)=\bar{\chi}$

- Pour tout $i$, si $i, i+1$ sont dans un même intervalle $J\left(\alpha_{j}\right)$, alors $\operatorname{Re}\left(\chi_{i}\right) \leqslant$ $\operatorname{Re}\left(\chi_{i+1}\right)$.

Notons $\sec _{P}(\bar{\chi})=\chi, c f$. section 7.3.1. On a :

$$
\operatorname{tr}_{\theta-c} \pi(f)=\sum_{\chi \in \mathcal{C}(\pi)} \operatorname{tr}_{\theta-c} \pi[\chi](f)
$$

où

$$
\operatorname{tr}_{\theta-c} \pi[\chi](f)=\sum_{\substack{\mathbf{P}=\mathbf{M N} \in \mathcal{P}^{\theta-s t} \\ \bar{x} \in \mathcal{C}_{P}(\pi), \sec _{P}(\bar{\chi})=\chi}}(-1)^{a_{P}^{\theta}-a_{G}^{\theta}} \operatorname{dim} V_{N}^{K_{M}}[\bar{\chi}] \operatorname{tr} \operatorname{Ind}_{B}^{M}(\chi)\left(\widehat{\chi}_{N}^{\theta} f^{P}\right)
$$

Pour $\chi \in \mathcal{C}(\pi)$, posons :

$$
\begin{aligned}
& \Sigma=\left\{i \in[1, n] ; \operatorname{Re}\left(\chi_{i}\right) \leqslant \operatorname{Re}\left(\chi_{i+1}\right)\right\} \\
& \Sigma_{=}=\left\{i \in \Sigma ; \operatorname{Re}\left(\chi_{i}\right)=\operatorname{Re}\left(\chi_{i+1}\right)\right\} \\
& \Sigma_{\neq}=\left\{i \in \Sigma ; \operatorname{Re}\left(\chi_{i}\right)<\operatorname{Re}\left(\chi_{i+1}\right)\right\}
\end{aligned}
$$

Pour $\Delta \subset \Sigma$, on définit le sous-groupe parabolique standard $\theta$-stable $\mathbf{P}_{\boldsymbol{\Delta}}$ de la même façon que dans le cas $\pi=\mathrm{St}_{2 h+1} \times \xi \mathrm{St}_{2 k+1} \times \xi \mathrm{St}_{1}$. Avec des arguments similaires que dans le cas $\pi=\mathrm{St}_{2 h+1} \times \xi \mathrm{St}_{2 k+1} \times \xi \mathrm{St}_{1}$, on peut exprimer la trace tordue compacte d'une fonction sphérique $f$ sous la forme suivante :

$$
\operatorname{tr}_{\theta-c} \pi(f)=\sum_{\chi \in \mathcal{C}(\pi)} \operatorname{tr}_{\theta-c} \pi[\chi](f)
$$


où

$$
\operatorname{tr}_{\theta-c} \pi[\chi](f)=\sum_{\Delta \subset \Sigma}(-1)^{a_{P_{\Delta}}^{\theta}-a_{G}^{\theta}} 2 \frac{\operatorname{card}^{\left.\operatorname{coup}_{P_{\Delta}}\left(\operatorname{res}_{P}(\chi)\right)\right)}}{2} \operatorname{tr} \operatorname{Ind}_{B}^{M_{\Delta}}(\chi)\left(\widehat{\chi}_{N_{\Delta}}^{\theta} f^{P_{\Delta}}\right)
$$

On a toujours l'égalité :

$$
a_{P_{\Delta}}^{\theta}-a_{G}^{\theta}=\operatorname{card}(\Delta)+n
$$

Quant au terme $2 \frac{\operatorname{card}\left(\operatorname{Coup}_{P_{\Delta}}\left(\operatorname{res}_{P_{\Delta}}(x)\right)\right)}{2}$, il est donné par le lemme suivant :

Lemme 8.22. - On a l'identité suivante :

$$
\frac{\operatorname{card}\left(\operatorname{Coup}_{P_{\Delta}}\left(\operatorname{res}_{P_{\Delta}}(\chi)\right)\right)}{2}=\frac{\operatorname{card}(\operatorname{Coup}(\chi))}{2}-\operatorname{card}\left(\Delta \cap \Sigma_{=}\right)
$$

Démonstration. - Soit $i \geqslant 1$ tel que $(i, i-1)$ soit une coupure de $\chi$. Soit $\alpha=$ $\left(\alpha_{1}, \ldots, \alpha_{2 r-1}\right)$ est la partition associée à $\mathbf{P}_{\Delta}$. Par définition de $\Sigma$, on ne peut pas avoir $\left\{e^{\prime \prime}(i), e^{\prime}(i-1)\right\}=J\left(\alpha_{j}\right)$ pour un $j$. Par conséquent, $(i, i-1)$ est une $P_{\Delta}$-coupure sauf si $e^{\prime}(i-1), e^{\prime \prime}(i-1)$ sont dans un même intervalle $J\left(\alpha_{j}\right)$ pour un certain $j$. L'ensemble des $i \geqslant 1$ tels que $e^{\prime}(i-1), e^{\prime \prime}(i-1) \in J\left(\alpha_{j}\right)$ pour un certain $j$ est en bijection avec $\Delta \cap \Sigma_{=}$, d'où le lemme.

On a défini les ensembles $\mathcal{L}(\Delta) \subset \mathbb{Z}^{n}, c f$. section 7.3.3. On dispose alors toujours de la formule 7.29 :

$$
\operatorname{tr} \operatorname{Ind}_{B}^{M_{\Delta}}(\chi)\left(\widehat{\chi}_{N_{\Delta}}^{\theta} f^{P_{\Delta}}\right)=\sum_{\boldsymbol{l} \in \mathbb{Z}^{n}} d_{\boldsymbol{l}} q^{-\langle\chi, l\rangle} \mathbf{1}_{\mathcal{L}(\Delta)}(\boldsymbol{l})
$$

L'égalité suivante se déduit de ce qui précède :

$$
\operatorname{tr}_{\theta-c} \pi[\chi](f)=2^{\frac{\operatorname{card}(\operatorname{Coup}(\chi))}{2}} \sum_{\boldsymbol{l} \in \mathbb{Z}^{n}}\left(\sum_{\Delta \subset \Sigma}(-1)^{\operatorname{card}(\Delta)+n} 2^{-\operatorname{card}\left(\Delta \cap \Sigma_{=}\right)} \mathbf{1}_{\mathcal{L}(\Delta)}(\boldsymbol{l})\right) d_{\boldsymbol{l}} q^{-\langle\chi, \boldsymbol{l}\rangle}
$$

Nous allons encore récrire la formule précédente sous une forme quelque peu différente. Pour $\Delta \subset \Sigma_{=}$, on a posé :

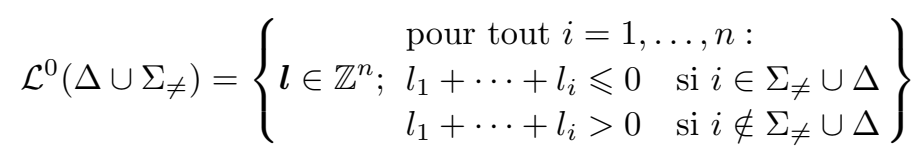

On a la formule combinatoire suivante :

Lemme 8.23. - On a l'identité :

$$
\sum_{\Delta \subset \Sigma}(-1)^{\operatorname{card}(\Delta)} 2^{-\operatorname{card}\left(\Delta \cap \Sigma_{=}\right)} \mathbf{1}_{\mathcal{L}(\Delta)}=2^{-\operatorname{card}\left(\Sigma_{=}\right)} \sum_{\Delta \subset \Sigma_{=}}(-1)^{\operatorname{card}\left(\Delta \cup \Sigma_{\neq}\right)} \mathbf{1}_{\mathcal{L}^{0}\left(\Delta \cup \Sigma_{\neq}\right)}
$$

Démonstration. - Écrivons : $\Delta=\Delta_{\neq} \cup \Delta_{=}$avec $\Delta_{\neq}=\Delta \cap \Sigma_{\neq}, \Delta_{=}=\Delta \cap \Sigma_{=}$. Le premier membre de l'égalité cherchée est donc égal à

$$
\sum_{\Delta_{=} \subset \Sigma_{=}}(-2)^{-\operatorname{card}\left(\Delta_{=}\right)} \sum_{\Delta_{\neq} \subset \Sigma_{\neq}}(-1)^{\operatorname{card}\left(\Delta_{\neq}\right)} \mathbf{1}_{\mathcal{L}\left(\Delta_{=} \cup \Delta_{\neq}\right)}
$$


Pour $\Delta_{=} \subset \Sigma_{=}$fixé, posons :

$$
\mathcal{G}\left(\Delta_{=}, \Sigma_{\neq}\right)=\left\{\begin{array}{c}
\text { pour tout } i=1, \ldots, n \\
\boldsymbol{l} \in \mathbb{Z}^{n} ; l_{1}+\cdots+l_{i} \leqslant 0 \quad \text { si } i \in \Sigma_{\neq} \\
l_{1}+\cdots+l_{i}>0 \text { si } i \notin \Sigma_{\neq} \cup \Delta_{=}
\end{array}\right\}
$$

On démontre facilement que :

$$
\sum_{\Delta_{\neq} \subset \Sigma_{\neq}}(-1)^{\operatorname{card}\left(\Delta_{\neq}\right)} \mathbf{1}_{\mathcal{L}\left(\Delta_{=} \cup \Delta_{\neq}\right)}=(-1)^{\operatorname{card}\left(\Sigma_{\neq}\right)} \mathbf{1}_{\mathcal{G}\left(\Delta_{=}, \Sigma_{\neq}\right)}
$$

Par ailleurs,

$$
\mathbf{1}_{\mathcal{G}\left(\Delta_{=}, \Sigma_{\neq}\right)}=\sum_{\Omega \subset \Delta_{=}} \mathbf{1}_{\mathcal{L}^{0}\left(\Omega \cup \Sigma_{\neq}\right)}
$$

On en déduit :

$$
\begin{aligned}
& \sum_{\Delta \subset \Sigma}(-1)^{\operatorname{card}(\Delta)} 2^{-\operatorname{card}\left(\Delta \cap \Sigma_{=}\right)} \mathbf{1}_{\mathcal{L}(\Delta)} \\
& =(-1)^{-\operatorname{card}\left(\Sigma_{\neq}\right)} \sum_{\Omega \subset \Sigma_{\neq}} \sum_{\substack{\Delta_{=}=\\
\Omega \subset \Delta_{=} \subset \Sigma_{=}}}(-2)^{\operatorname{card}\left(\Delta_{=}\right)} \cdot \mathbf{1}_{\mathcal{L}^{0}\left(\Omega \cup \Sigma_{\neq}\right)}
\end{aligned}
$$

L'égalité cherchée vient alors de la formule ci-dessus et les égalités suivantes :

$$
\begin{aligned}
\sum_{\substack{\Delta_{=} \\
\Omega \subset \Delta_{=} \subset \Sigma_{=}}}(-2)^{-\operatorname{card}\left(\Delta_{=}\right)} & =(-2)^{-\operatorname{card}(\Omega)} \sum_{\Delta \subset \Sigma_{=} \backslash \Omega}(-2)^{\operatorname{card}(\Delta)} \\
& =(-2)^{-\operatorname{card}(\Omega)}\left(1-2^{-1}\right)^{\operatorname{card}\left(\Sigma_{=} \backslash \Omega\right)} \\
& =(-1)^{\operatorname{card}(\Omega)} 2^{-\operatorname{card}\left(\Sigma_{=}\right)}
\end{aligned}
$$

Posons :

$$
\operatorname{Dist}_{\Delta}(f)=2^{\frac{\operatorname{card}(\operatorname{Coup}(\chi))}{2}-\operatorname{card}\left(\Sigma_{=}\right)}(-1)^{\operatorname{card}\left(\Delta \cup \Sigma_{\neq}\right)+n} \sum_{\boldsymbol{l} \in \mathbb{Z}^{n}} d_{\boldsymbol{l}} q^{-\langle\chi, \boldsymbol{l}\rangle} \mathbf{1}_{\mathcal{L}^{0}\left(\Delta \cup \Sigma_{\neq}\right)}(\boldsymbol{l})
$$

On a alors l'identité :

$$
\operatorname{tr}_{\theta-c} \pi[\chi](f)=\sum_{\Delta \subset \Sigma=} \operatorname{Dist}_{\Delta}(f)
$$

\subsection{Transformation de Satake de $\operatorname{tr}_{\theta-c} \pi$}

8.3.1. Les termes Dist $_{\Delta} \cdot-$ Pour $\Delta \subset \Sigma_{=}$, on dira qu'un $n$-uplet $\delta=\left(\delta_{1}, \ldots, \delta_{n}\right)$ est bon pour $\Delta$ s'il satisfait les conditions suivantes :

$$
\begin{array}{ll}
\delta_{1}, \ldots, \delta_{n} & \text { réels assez proches de } 1 ; \\
\delta_{i}<\delta_{i+1} & \text { si } i \in \Delta \\
\delta_{i}>\delta_{i+1} & \text { si } i \in \Sigma_{=} \backslash \Delta .
\end{array}
$$

Rappelons aussi que :

$$
\widehat{T}_{u, \delta}=\left\{\left(x_{1}, \ldots, x_{n}\right) \in \mathbb{C}^{n} ; \text { pour tout } i,\left|x_{i}\right|=\delta_{i}\right\}
$$


On rappelle la définition de la fonction $Q_{\chi}$ :

$$
Q_{\chi}\left(x_{1}, \ldots, x_{n}\right)=\frac{1}{1-q^{\chi_{n}} x_{n}} \prod_{i=1}^{n-1} \frac{1}{1-q^{\chi_{i}-\chi_{i+1}} x_{i} x_{i+1}^{-1}}
$$

En suivant la méthode décrite dans le cas $\pi=\mathrm{St}_{2 h+1} \times \xi \mathrm{St}_{2 k+1} \times \xi \mathrm{St}_{1}$, on peut démontrer le résultat suivant :

Proposition 8.24. - Soit $\Delta \subset \Sigma_{=}$, et soit $\delta$ un bon $n$-uplet pour $\Delta$. Alors, pour tout $f \in \mathcal{H}$, on a l'identité :

$$
\begin{aligned}
& \operatorname{Dist}_{\Delta}(f) \\
& \quad=2^{\frac{\operatorname{card}(\operatorname{Coup}(\chi))}{2}-\operatorname{card}\left(\Sigma_{=}\right)} \int_{\widehat{T}_{u, \delta}} p(S(f))\left(x_{1}, \ldots, x_{n}\right) Q_{\chi}\left(x_{1}, \ldots, x_{n}\right) d^{\times} x_{1} \cdots d^{\times} x_{n}
\end{aligned}
$$

8.3.2. Élimination des pôles. - Tout d'abord, remarquons que les fonctions $Q_{\chi}$ ont des pôles au voisinage de $\widehat{T}_{u}$ précisément en $x_{i}=x_{i+1}$ pour les $i \in \Sigma_{=}$et éventuellement en $x_{n}=-1$. On remarquera également qu'on a toujours $1 \notin \Sigma_{=}$. Soient $i \in \Sigma_{=}, \Delta \subset \Sigma_{=}$tels que $i \notin \Delta$. Supposons que $i \neq n$, le cas $i=n$ se démontre de façon similaire. Soit $\delta=\left(\delta_{1}, \ldots, \delta_{n}\right)$ un bon $n$-uplet pour $\Delta$. Alors le $n$-uplet $\delta^{\prime}=s_{i} \delta=\left(\delta_{1}, \ldots, \delta_{i-1}, \delta_{i+1}, \delta_{i}, \delta_{i+2}, \ldots, \delta_{n}\right)$ est bon pour $\Delta \cup\{i\}$. D'après ce qui précède, on a :

$$
\begin{aligned}
& \operatorname{Dist}_{\Delta \cup\{i\}}(f)=2^{\frac{\operatorname{card}(\operatorname{Coup}(\chi))}{2}-\operatorname{card}\left(\Sigma_{=}\right)} \int_{\widehat{T}_{u, \delta^{\prime}}} p(S(f))\left(x_{1}, \ldots, x_{n}\right) \\
& Q_{\chi}\left(x_{1}, \ldots, x_{n}\right) d^{\times} x_{1} \cdots d^{\times} x_{n} \\
&=2^{\frac{\operatorname{card}(\operatorname{Coup}(\chi))}{2}-\operatorname{card}\left(\Sigma_{=}\right)} \int_{\widehat{T}_{u, \delta}} p(S(f))\left(x_{1}, \ldots, x_{n}\right) \\
& \cdot Q_{\chi}^{s_{i}}\left(x_{1}, \ldots, x_{n}\right) d^{\times} x_{1} \cdots d^{\times} x_{n}
\end{aligned}
$$

On en tire :

$$
\begin{aligned}
\operatorname{Dist}_{\Delta}(f)+\operatorname{Dist}_{\Delta \cup\{i\}}(f)= & 2^{\frac{\operatorname{card}(\operatorname{Coup}(\chi))}{2}-\operatorname{card}\left(\Sigma_{=}\right)} \int_{\widehat{T}_{u, \delta}} p(S(f))\left(x_{1}, \ldots, x_{n}\right) \\
& \cdot\left(Q_{\chi}\left(x_{1}, \ldots, x_{n}\right)+Q_{\chi}^{s_{i}}\left(x_{1}, \ldots, x_{n}\right)\right) d^{\times} x_{1} \cdots d^{\times} x_{n}
\end{aligned}
$$

Or, comme $i \in \Sigma_{=}$et par hypothèse $i \neq n$, on a $: \chi_{i}=\chi_{i+1}$ de sorte que $Q_{\chi}+Q_{\chi}^{s_{i}}=$ $Q_{\chi}+Q_{s_{i} \chi}^{s_{i}}$. On sait que cette fonction n'a pas de pôle en $x_{i}=x_{i+1}$ (cf. section 7.4.3). Posons :

$$
\begin{aligned}
& Q_{\chi}^{\mathrm{sym}}=\frac{1}{\left|W\left(C_{n}\right)\right|} \sum_{w \in W\left(C_{n}\right)} Q_{\chi}^{w} \\
& Q_{\pi}=\sum_{\chi \in \mathcal{C}(\pi)} 2^{\frac{\operatorname{card}(\operatorname{Coup}(\chi))}{2}} Q_{\chi}^{\mathrm{sym}}
\end{aligned}
$$

Les remarques ci-dessus, jointes à un raisonnement analogue à celui précédant le théorème 7.39, permettent de démontrer le résultat suivant : 
THÉORÈmE 8.25. - La fonction $Q_{\pi}$ est sans pôle au voisinage de $\widehat{T}_{u}$. Pour tout $f \in \mathcal{H}$ on a les égalités suivantes :

$$
\begin{aligned}
\operatorname{tr}_{\theta-c}[\chi](f) & =2^{\frac{\operatorname{card}(\operatorname{Coup}(\chi))}{2}} \int_{\widehat{T}_{u}} p(S(f))\left(x_{1}, \ldots, x_{n}\right) Q_{\chi}^{\mathrm{sym}}\left(x_{1}, \ldots, x_{n}\right) d^{\times} x_{1} \cdots d^{\times} x_{n} \\
\operatorname{tr}_{\theta-c} \pi(f) & =\int_{\widehat{T}_{u}} p(S(f))\left(x_{1}, \ldots, x_{n}\right) Q_{\pi}\left(x_{1}, \ldots, x_{n}\right) d^{\times} x_{1} \cdots d^{\times} x_{n}
\end{aligned}
$$

8.3.2.1. Interprétation du terme $2^{\frac{\operatorname{card}(\operatorname{Coup}(\chi))}{2}}$. - Soit $\chi \in \mathcal{C}(\pi)$, et soit $w \in\left[W / W^{L}\right]$ tel que $w \eta=\chi$. Par des arguments similaires à ceux de la démonstration du lemme 8.9 , on obtient l'égalité :

$$
\operatorname{card}\left\{w^{\prime} \in\left[W / W^{L}\right] ; w^{\prime} w^{-1} \chi=\chi, \theta\left(w^{\prime} w^{-1}\right)=w^{\prime} w^{-1}\right\}=2^{\frac{\operatorname{card}(\operatorname{Coup}(\chi))}{2}}
$$

En remplaçant $w^{\prime} w^{-1}$ par $w^{\prime}$ on a :

$$
\operatorname{card}\left\{w^{\prime} \in W ; w^{\prime} w \in\left[W / W^{L}\right], w^{\prime} w \eta=w \eta, \theta\left(w^{\prime}\right)=w^{\prime}\right\}=2^{\frac{\operatorname{card}(\operatorname{Coup}(\chi))}{2}}
$$

Le nombre des $w^{\prime} \in\left[W / W^{L}\right]$ tels que $w^{\prime} \eta=\chi$ est égal à

$$
\operatorname{card}\left\{w^{\prime} \in\left[W / W^{L}\right] ; w^{\prime} \eta=\chi\right\}=\operatorname{card}\left\{w^{\prime} \in W ; w^{\prime} w \in\left[W / W^{L}\right], w^{\prime} w \eta=w \eta\right\}
$$

Par suite,

$$
2^{\frac{\operatorname{card}(\operatorname{Coup}(\chi))}{2}}=\sum_{\substack{w \in\left[W / W^{L}\right] \\ w \eta=\chi}} \frac{\operatorname{card}\left\{w^{\prime} \in W ; w^{\prime} w \in\left[W / W^{L}\right], w^{\prime} w \eta=w \eta, \theta\left(w^{\prime}\right)=w^{\prime}\right\}}{\operatorname{card}\left\{w^{\prime} \in W ; w^{\prime} w \in\left[W / W^{L}\right], w^{\prime} w \eta=w \eta\right\}}
$$

On en déduit :

Proposition 8.26. - On a l'égalité :

$$
Q_{\pi}=\sum_{\substack{w \in\left[W / W^{L}\right], \theta(w \eta)=w \eta}} \frac{\operatorname{card}\left\{w^{\prime} \in W ; w^{\prime} w \in\left[W / W^{L}\right], w^{\prime} w \eta=w \eta, \theta\left(w^{\prime}\right)=w^{\prime}\right\}}{\operatorname{card}\left\{w^{\prime} \in W ; w^{\prime} w \in\left[W / W^{L}\right], w^{\prime} w \eta=w \eta\right\}} Q_{w \eta}^{\text {sym }}
$$





\title{
CHAPITRE 9
}

\author{
LE CAS $\pi=\mathrm{St}_{2 h+1} \times \mathrm{St}_{2 k+1} \times \mathrm{St}_{1}$
}

Il s'agit du cas où $\left(\alpha^{+}, \alpha^{-}, \beta\right)=((2 h+1,2 k+1,1), \varnothing, \varnothing)$, où $h>k>0$, $h+k+1=n$.

Notons que, dans ce cas, le groupe parabolique $\mathbf{R}=\mathbf{L V}$ est associé à la partition $(2 h+1,2 k+1,1)$. Le groupe de Weyl $W^{L}$ est donc isomorphe à $\mathfrak{S}_{2 h+1} \times \mathfrak{S}_{2 k+1} \times \mathfrak{S}_{1}$. On note, comme c'est usuel, $V$ l'espace de $\pi$ et $A \in \operatorname{End}_{\mathbb{C}}(V)$ l'opérateur d'entrelacement entre $\pi$ et $\pi^{\theta}$, normalisé comme en 2.3. La méthode employée ici et les résultats obtenus sont semblables au cas précédent.

\subsection{Le module de Jacquet $\pi_{U}$}

Dans ce cas, on a :

$$
\eta=(h, \ldots,-h, k, \ldots,-k, 0)
$$

Posons $I(h)=\left[a_{h}, a_{-h}\right]=[1,2 h+1], I(k)=\left[b_{k}, b_{-k}\right]=[2 h+2,2 h+2 k+2]$. On a donc $\eta_{a_{0}}=\eta_{b_{0}}=\eta_{2 n+1}=0$. On définit l'ensemble $\mathcal{C}(\pi)$ de la même manière que pour $\pi=\mathrm{St}_{1} \times \xi \mathrm{St}_{2 h+1} \times \xi \mathrm{St}_{2 k+1}$. Soit $\chi \in \mathcal{C}(\pi)$, calculons $\operatorname{tr}_{\theta-c} \pi_{U}[\chi]$.

\subsubsection{Coupure dominante, construction de $\varrho$}

9.1.1.1. Définition de $i_{\max }$. — Dans le cas présent, parce qu'il y a trois zéros parmi les $\eta_{i}$, nous sommes obligés de modifier légèrement la définition des coupures. Pour $h \geqslant i \geqslant-h$, on avait posé : $E(i)=\left\{j, \chi_{j}=i\right\}$. Écrivons :

$$
E(i)= \begin{cases}\{e(i)\} & \text { pour } h \geqslant|i|>k \\ \left\{e^{\prime}(i)<e^{\prime \prime}(i)\right\} & \text { pour } k \geqslant|i|>0 \\ \left\{e^{\prime}(0)<n+1<e^{\prime \prime}(0)\right\} & \text { pour } i=0\end{cases}
$$

La coupure dominante est définie comme suit. Il y a deux cas possibles :

- Il existe un entier $i, k+1 \geqslant i \geqslant 2$ tels que $e^{\prime \prime}(i)<e^{\prime}(i-1)$ (avec la convention $\left.e^{\prime \prime}(k+1)=e(k+1)\right)$. Nous définissons $i_{\max }$ comme le plus grand entier vérifiant cette propriété;

- Dans le cas contraire, on pose $i_{\max }=1$. 


\subsubsection{La construction de @ dépend de $i_{\max }$}

Cas $i_{\max }>1$. - La représentation $\varrho$ est définie comme dans le cas $\pi=$ $\mathrm{St}_{1} \times \xi \mathrm{St}_{2 h+1} \times \xi \mathrm{St}_{2 k+1}$. Concrètement, posons $: d=n-e^{\prime \prime}\left(i_{\max }\right)=2 i_{\max }-1$. Soit $\mathbf{Q}=\mathbf{M}_{\mathbf{Q}} \mathbf{N}_{\mathbf{Q}}$ le parabolique standard $\theta$-stable associé à la partition $(n-d, 2 d+1, n-d)$. On définit alors :

$$
\varrho=\left(\mathrm{St}_{h, i_{\max }} \times \mathrm{St}_{k, i_{\max }}\right) \otimes\left(\mathrm{St}_{d} \times \mathrm{St}_{1} \times \mathrm{St}_{d}\right) \otimes\left(\mathrm{St}_{-i_{\max },-k} \times \mathrm{St}_{-i_{\max },-h}\right)
$$

Cas $i_{\max }=1$. - Dans ce cas, on pose : $d=n-e^{\prime}(1)$. On note $\mathbf{Q}=\mathbf{M}_{\mathbf{Q}} \mathbf{N}_{\mathbf{Q}}$ le parabolique standard $\theta$-stable paramétré par la partition

$$
(\underbrace{1, \ldots, 1}_{n-d}, 2 d+1, \underbrace{1, \ldots, 1}_{n-d})
$$

On définit :

$$
\varrho=\chi_{1} \otimes \cdots \otimes \chi_{n-d} \otimes\left(\mathrm{St}_{1} \times \mathrm{St}_{2 d-1} \times \mathrm{St}_{1}\right) \otimes \chi_{2 n+d-l} \otimes \cdots \otimes \chi_{2 n+1}
$$

Les propriétés de $\varrho$ (voir la proposition 8.6) restent valables. L'opérateur $A$ est toujours de la forme $f \mapsto f^{\theta}$. On obtient alors la formule de la proposition 8.8. Rappelons cidessous l'énoncé. Soit $\mathbf{P}=\mathbf{M}_{\mathbf{P}} \mathbf{N}_{\mathbf{P}}$ le parabolique standard $\theta$-stable correspondant à la partition

- $\left(h-i_{\max }+1, k-i_{\max }+1, d, 1, d, k-i_{\max }+1, h-i_{\max }+1\right)$ si $i_{\max }>1$

- $(1, \ldots, 1,1,2 d-1,1,1, \ldots, 1) \quad$ si $i_{\max }=1$

Proposition 9.1. - Soit $f$ une fonction localement constante, à support compact sur T. On a l'identité :

$$
\operatorname{tr}_{\theta} \pi_{U}[\chi](f)=\operatorname{card}\left\{w \in\left[W^{M_{Q}} / W^{M_{P}}\right] ; w \chi=\chi, \theta(w)=w\right\} \int_{T} \chi(t) f(t) d t
$$

9.1.2. Coupures revisitées, formule pour $\operatorname{tr}_{\theta-c} \pi[\chi]$. - La modification de la définition des coupures est motivée par la condition $\theta(w)=w$ dans la formule 9.2. Nous distinguons deux cas :

Cas $i_{\max }>1$. - On peut définir l'ensemble $\operatorname{Coup}(\chi)$ des coupures de $\chi$ comme dans le cas $\pi=\mathrm{St}_{1} \times \xi \mathrm{St}_{2 h+1} \times \xi \mathrm{St}_{2 k+1}$. Autrement dit, un couple $(i, i-1)$ sera qualifié de coupure pour $\chi$ si l'égalité suivante a lieu :

$$
e^{\prime \prime}(i)<e^{\prime}(i-1)
$$

cela toujours avec la convention $e^{\prime \prime}(k+1)=e(k+1)$.

Cas $i_{\max }=1$. - On pose tout simplement $\operatorname{Coup}(\chi)=\{(1,0),(0,-1)\}$.

On a l'égalité suivante, qui est valable dans les deux cas :

$$
\operatorname{card}\left\{w \in\left[W^{M_{Q}} / W^{M_{P}}\right] ; w \chi=\chi, \theta(w)=w\right\}=2^{\frac{\operatorname{card}(\operatorname{Coup}(\chi))}{2}}
$$

En appliquant cette formule à la formule 9.2, on obtient : 
Corollaire 9.3. - Soit $f$ une fonction lisse, à support compact sur le tore diagonal $T$ de $G$. On a alors l'identité :

$$
\operatorname{tr}_{\theta} \pi_{U}[\chi](f)=2^{\frac{\operatorname{card}(\operatorname{Coup}(\chi))}{2}} \int_{T} \chi(t) f(t) d t
$$

Ce n'est autre que le corollaire 8.11.

\subsection{Les modules de Jacquet $\pi_{N}$, où $\mathbf{P}=\mathbf{M N} \in \mathcal{P}^{\theta}$}

Fixons un parabolique standard $\theta$-stable $\mathbf{P}$, et soit $\alpha=\left(\alpha_{1}, \ldots, \alpha_{2 r-1}\right)$ la partition qui le paramétrise. On peut toujours supposer que les intervalles $J\left(\alpha_{i}\right)$ sont de longueur au plus 2 sauf $J\left(\alpha_{r}\right)$ qui est peut être de longueur 3.

9.2.1. Section $\sec _{P}$. - On définit l'ensemble $\mathcal{C}_{P}(\pi)$ comme dans les cas précédents. On définit de même une section $\mathcal{C}_{P}(\pi) \longrightarrow \mathcal{C}(\pi)$ : soit $\bar{\chi} \in \mathcal{C}_{P}(\pi)$, alors $\sec _{P}(\bar{\chi})$ est l'unique élément de $\mathcal{C}(\pi)$ avec les propriétés :

$-\chi \in \mathcal{C}(\pi)$ est de restriction $\bar{\chi}$

$-\chi_{i} \leqslant \chi_{i+1}$ si $(i, i+1)$ est de la forme $J\left(\alpha_{j}\right)$ pour $j=1, \ldots, 2 r-1$;

$-\chi_{n} \leqslant \chi_{n+1}=0 \leqslant \chi_{n+2}$ si $\{n, n+1, n+2\}$ est de la forme $J\left(\alpha_{r}\right)$.

9.2.2. $P$-coupures. - Fixons un $\bar{\chi} \in \mathcal{C}_{P}(\pi)$, et soit $\chi=\sec _{P}(\bar{\chi})$. La définition des $P$-coupures de $\bar{\chi}$ dépend de $i_{\max }$ (la coupure dominante de $\chi$ ):

Cas $i_{\max }>1$. - Nous définissons alors l'ensemble $\operatorname{Coup}_{P}(\bar{\chi})$ des $P$-coupures de $\bar{\chi}$ de façon suivante. Une coupure $(i, i-1)$ de $\chi$ avec $i \geqslant 1$ est appelée $P$-coupure de $\bar{\chi}$ si les conditions suivantes sont satisfaites :

- $\left\{e^{\prime \prime}(i), e^{\prime}(i-1)\right\}$ n'est pas de la forme $J\left(\alpha_{l}\right), l=1, \ldots, 2 r-1$;

- $\left\{e^{\prime}(i-1), e^{\prime \prime}(i-1)\right\}$ n'est pas de la forme $J\left(\alpha_{l}\right), l=1, \ldots, 2 r-1$;

- $\left\{e^{\prime}(i-1), e^{\prime \prime}(i-1), n+1\right\}$ n'est pas de la forme $J\left(\alpha_{r}\right)$.

On remarquera que la première condition est automatiquement vérifiée parce qu'on a choisi $\chi$ comme section de $\bar{\chi}$. Par symétrie, si $(i, i-1)$ est une $P$-coupure de $\bar{\chi}$ avec $i \geqslant 1$ on dit aussi que $(-i+1,-i)$ est une $P$-coupure de $\bar{\chi}$.

Cas $i_{\max }=1$. - On pose :

$$
\operatorname{Coup}_{P}(\bar{\chi})= \begin{cases}\{(1,0),(0,-1)\} & \text { si }\left(e^{\prime}(0), n+1, e^{\prime \prime}(0)\right) \neq J\left(\alpha_{r}\right) \\ \varnothing & \text { si }\left(e^{\prime}(0), n+1, e^{\prime \prime}(0)\right)=J\left(\alpha_{r}\right)\end{cases}
$$

\subsubsection{Construction de $\varrho$ lorsque $\operatorname{Coup}_{P}(\bar{\chi})$ est non vide et $i_{\max }>1$}

Dans ce cas, on peut parler de $P$-coupure dominante de $\bar{\chi}$. On applique ensuite la construction décrite dans le cas $\pi=\mathrm{St}_{2 h+1} \times \xi \mathrm{St}_{2 k+1} \times \xi \mathrm{St}_{1}$.

On a l'égalité :

$$
\operatorname{tr}_{\theta} \pi_{N}[\bar{\chi}](f)=2^{\frac{\operatorname{card}\left(\operatorname{Coup}_{P}(\bar{\chi})\right)}{2}} \operatorname{tr} \operatorname{Ind}_{B}^{M}(\chi)(f)
$$


9.2.4. Cas où $\operatorname{Coup}_{P}(\bar{\chi})$ est non vide et $i_{\max }=1$. - On va montrer que l'égalité 9.5 reste encore valable. Dans ce cas, on montre par la filtration de BernsteinZelevinsky que la dimension de $V_{N}^{K_{M}}[\bar{\chi}]$ est égale à 2 , de même que l'espace $V_{U}[\chi]$. D'autre part, d'après notre étude du module de Jacquet $\pi_{U}$, on sait que l'opérateur d'entrelacement $A$ agit trivialement sur la composante $V_{U}[\chi]$. Il nous suffit, pour prouver l'égalité 9.5 , de montrer que la composition de

$$
V_{N} \longrightarrow V_{U} \longrightarrow V_{U}[\chi]
$$

induit un isomorphisme entre $V_{N}^{K_{M}}[\bar{\chi}]$ et $V_{U}[\chi]$.

Rappelons que la partition associée à $\mathbf{P}$ est notée par $\alpha=\left(\alpha_{1}, \alpha_{2}, \ldots, \alpha_{2 r-1}\right)$, le sous-groupe de Lévi de $P$ est donc de la forme

$$
M=G L\left(\alpha_{1}, F\right) \times G L\left(\alpha_{2}, F\right) \times \cdots \times G L\left(\alpha_{2 r-1}, F\right)
$$

On vérifie d'après les définitions que dans ce cas $\alpha_{r}=1$. Par hypothèse, $(1,0),(0,-1)$ sont les uniques coupures de $\chi$ (et qui sont également les $P$-coupures de $\bar{\chi}$ ). Montrons que si $\alpha_{i}=2$ et si on écrit $J\left(\alpha_{i}\right)=\{j, j+1\}$ alors $\chi_{j} \neq \chi_{j+1}$, ou ce qui revient au même $\chi_{j}<\chi_{j+1}$, puisque $\chi_{j} \leqslant \chi_{j+1}$ par le choix de la section. En effet, si $\chi_{j}=\chi_{j+1}$ il y a forcément une coupure entre $\chi_{j-1}$ et $\chi_{j}$ de même qu'entre $\chi_{j+1}$ et $\chi_{j+2}$, ce qui implique que $\chi_{j}=\chi_{j+1}=0$, parce que $i_{\max }=1$. Par symétrie, $\chi_{2 r+1-j}=\chi_{2 r+2-j}=0$, et il y a au moins quatre zéros parmi les $\chi_{i}$ (au lieu de 3 maximum), ce qui est une contradiction.

On peut montrer, par application de la filtration de Bernstein-Zelevinsky, que le module $V_{N}[\bar{\chi}]$ est une représentation de $M$ ayant les propriétés suivantes :

- c'est une représentation de longueur finie de $M$;

- les sous-quotients irréductibles sont de la forme $\rho_{1} \otimes \rho_{2} \otimes \cdots \otimes \rho_{2 r+1}$ avec

- Si $\alpha_{i}=1, J\left(\alpha_{i}\right)=\{j\}$, alors $\rho_{i}=\chi_{i}$;

- Si $\alpha_{i}=2, J\left(\alpha_{i}\right)=\{j, j+1\}$, et $\chi_{j}-\chi_{j+1} \neq-1$, alors $\rho_{i}=\chi_{j} \times \chi_{j+1}$;

- Si $\alpha_{i}=2, J\left(\alpha_{i}\right)=\{j, j+1\}$, et $\chi_{j}-\chi_{j+1}=-1$, alors soit $\rho_{i}=|\operatorname{det}|^{\frac{\chi_{j}+\chi_{j+1}}{2}}$, soit $|\operatorname{det}| \frac{\chi_{j}+\chi_{j+1}}{2} \mathrm{St}_{2}$.

Rappelons la propriété de $\chi$, qui est essentielle pour le lemme ci-dessous : si $\alpha_{i}=2$ et $J\left(\alpha_{i}\right)=\{j, j+1\}$, alors on a : $\chi_{j}<\chi_{j+1}$.

LEMME 9.6. - Soit $(\rho, E)$ une représentation de $M$ vérifiant les conditions ci-dessus. Alors la composition de $E \longrightarrow E_{U} \longrightarrow E_{U}[\chi]$ induit une bijection entre $E^{K_{M}}[\bar{\chi}]$ et $E_{U}[\chi]$.

Démonstration. - On est immédiatement ramené au problème suivant : soit $\chi=$ $\left(\chi_{1}, \chi_{2}\right)$ un caractère non ramifié du tore diagonal de $G L(2)$, avec $\chi_{1}<\chi_{2}$, soit $(\rho, E)$ une représentation de longueur finie de $G L(2)$ dont tous les sous-quotients irréductibles sont sous-quotients de $\operatorname{Ind}(\chi)$. Alors la composition $E \longrightarrow E_{U} \longrightarrow E_{U}[\chi]$ 
induit une bijection $E^{K} \mapsto E_{U}[\chi]$ (avec les notations évidentes). Puisque les foncteurs $E \mapsto E_{U}, E_{U}[\chi], E^{K}$ sont exacts, on est amené au cas où $E$ est irréductible.

Si $\chi_{1}-\chi_{2} \neq-1$, alors le module de Jacquet $\rho_{U}$ est la somme directe de deux caractères non ramifiés : $\left(\chi_{1}, \chi_{2}\right)$ et $\left(\chi_{2}, \chi_{1}\right)$. Il suffit de montrer que l'image de $E^{K}$ dans $E_{U}[\chi]$ est non nulle. Si elle était nulle, elle serait incluse dans $E_{U}\left[\left(\chi_{2}, \chi_{1}\right)\right]$. Mais un simple calcul sur l'algèbre de Hecke-Iwahori de $G L(2)$, en employant les résultats du paragraphe 2.3.5, montre que ce n'est pas le cas.

Si $\chi_{1}-\chi_{2}=-1$, alors soit $\rho=|\operatorname{det}|^{\frac{\chi_{1}+\chi_{2}}{2}}$ soit $\rho=|\operatorname{det}|^{\frac{\chi_{1}+\chi_{2}}{2}} \operatorname{St}_{2}$. Mais si $\rho=$ $|\operatorname{det}| \frac{\chi_{1}+\chi_{2}}{2}$ alors $\rho_{U}=\left(\chi_{1}, \chi_{2}\right)=\chi$ et $E^{K_{M}}[\bar{\chi}] \longrightarrow E_{U}[\chi]$ est un isomorphisme entre deux droites, si $\rho=|\operatorname{det}|^{\frac{\chi_{1}+\chi_{2}}{2}} \mathrm{St}_{2}$ alors $\rho_{U}=\left(\chi_{2}, \chi_{1}\right)$ et $E^{K_{M}}[\bar{\chi}]=E_{U}[\chi]=0$.

9.2.5. Cas où $\operatorname{Coup}_{P}(\bar{\chi})$ est vide. - On peut montrer que la dimension de $V_{N}^{K_{M}}[\bar{\chi}]$ est égale à 1 . On montre d'après notre normalisation de l'opérateur d'entrelacement $A$ qu'il agit trivialement sur cet espace. Il s'ensuit immédiatement que la formule 9.5 est valable dans ce cas.

\subsection{Formule pour $\operatorname{tr}_{\theta-c} \pi$}

On peut écrire, comme dans le cas $\pi=\mathrm{St}_{1} \times \xi \mathrm{St}_{2 h+1} \times \xi \mathrm{St}_{2 k+1}$, pour $f \in \mathcal{H}(G)$ :

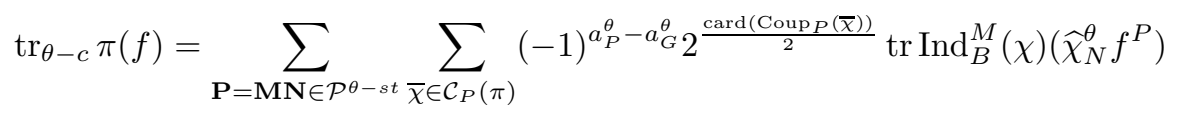

où pour tout $\bar{\chi} \in \mathcal{C}_{P}(\pi)$, on a $\chi=\sec _{P}(\bar{\chi})$. On définit les ensembles $\Sigma, \Sigma_{=}, \Sigma_{\neq}$de façon suivante :

$$
\begin{aligned}
& \Sigma=\left\{i \in[1, n] ; \chi_{i} \leqslant \chi_{i+1}\right\} \\
& \Sigma_{=}=\left\{i \in \Sigma ; \chi_{i}=\chi_{i+1}\right\} \\
& \Sigma_{\neq}=\left\{i \in \Sigma ; \chi_{i}<\chi_{i+1}\right\}
\end{aligned}
$$

Comme dans le cas $\pi=\mathrm{St}_{1} \times \xi \mathrm{St}_{2 h+1} \times \xi \mathrm{St}_{2 k+1}$, on récrit la formule ci-dessus en une somme sur les $\chi \in \mathcal{C}(\pi)$. Pour ce faire, il suffit de répéter les arguments du cas cité. Nous nous contentons de donner les résultats sans entrer dans les détails. On a l'égalité :

$$
\operatorname{tr}_{\theta-c} \pi(f)=\sum_{\chi \in \mathcal{C}(\pi)} \operatorname{tr}_{\theta-c} \pi[\chi](f)
$$

où

$$
\operatorname{tr}_{\theta-c} \pi[\chi](f)=\sum_{\Delta \subset \Sigma}(-1)^{a_{P_{\Delta}}^{\theta}-a_{G}^{\theta}} 2 \frac{\operatorname{card}\left(\operatorname{Coup}_{P_{\Delta}}(\bar{\chi})\right)}{2} \operatorname{tr} \operatorname{Ind}_{B}^{M_{\Delta}}(\chi)\left(\widehat{\chi}_{N_{\Delta}}^{\theta} f^{P_{\Delta}}\right)
$$

Posons, pour $\Delta \subset \Sigma_{=}$:

$$
\mathcal{L}^{0}\left(\Delta \cup \Sigma_{\neq}\right)=\left\{\begin{array}{ll} 
& \text { pour tout } i=1, \ldots, n: \\
\boldsymbol{l} \in \mathbb{Z}^{n} ; & l_{1}+\cdots+l_{i} \leqslant 0 \quad \text { si } i \in \Sigma_{\neq} \cup \Delta \\
& l_{1}+\cdots+l_{i}>0 \quad \text { si } i \notin \Sigma_{\neq} \cup \Delta
\end{array}\right\}
$$


Écrivons :

$$
S(f)\left(X_{1}, \ldots, X_{2 n+1}\right)=\sum_{\boldsymbol{m}=\left(m_{1}, \ldots, m_{2 n+1}\right) \in \mathbb{Z}^{2 n+1}} c_{\boldsymbol{m}} X_{1}^{m_{1}} X_{2}^{m_{2}} \cdots X_{2 n+1}^{m_{2 n+1}}
$$

et posons, pour $\boldsymbol{l}=\left(l_{1}, \cdots, l_{n}\right) \in \mathbb{Z}^{n}$ :

$$
\begin{gathered}
d_{\boldsymbol{l}}=\sum_{\substack{\boldsymbol{m}=\left(m_{1}, \ldots, m_{2 n+1}\right) \in \mathbb{Z}^{2 n+1} \\
\text { pour tout } i=1, \ldots, n: \\
m_{i}-m_{2 n+2-i}=l_{i}}} c_{\boldsymbol{m}} \\
\langle\chi, \boldsymbol{l}\rangle=\chi_{1} l_{1}+\chi_{2} l_{2}+\cdots+\chi_{n} l_{n}
\end{gathered}
$$

Posons, pour $\Delta \subset \Sigma_{=}$:

$$
\operatorname{Dist}_{\Delta}(f)=2^{\frac{\operatorname{card}(\operatorname{Coup}(\chi))}{2}-\operatorname{card}\left(\Sigma_{=}\right)} \sum_{\boldsymbol{l} \in \mathbb{Z}^{n}}(-1)^{\operatorname{card}\left(\Delta \cup \Sigma_{\neq}\right)} d_{\boldsymbol{l}} q^{-\langle\chi, \boldsymbol{l}\rangle} \mathbf{1}_{\mathcal{L}^{0}\left(\Delta \cup \Sigma_{\neq}\right)}(\boldsymbol{l})
$$

On a alors l'égalité :

$$
\operatorname{tr}_{\theta-c} \pi[\chi](f)=\sum_{\Delta \subset \Sigma_{=}} \operatorname{Dist}_{\Delta}(f)
$$

\subsection{Transformation de Satake de $\operatorname{tr}_{\theta-c} \pi$}

En empruntant les arguments du cas $\pi=\mathrm{St}_{2 h+1} \times \xi \mathrm{St}_{2 k+1} \times \xi \mathrm{St}_{1}$, on obtient :

ThÉORÈme 9.7. - Pour tout $f \in \mathcal{H}$, on a les égalités suivantes :

$$
\begin{aligned}
& \operatorname{tr}_{\theta-c}[\chi](f)=2^{\frac{\operatorname{card}(\operatorname{Coup}(\chi))}{2}} \int_{\widehat{T}_{u}} p(S(f))\left(x_{1}, \ldots, x_{n}\right) Q_{\chi}^{\mathrm{sym}}\left(x_{1}, \ldots, x_{n}\right) d^{\times} x_{1} \cdots d^{\times} x_{n} \\
& \operatorname{tr}_{\theta-c} \pi(f)=\int_{\widehat{T}_{u}} p(S(f))\left(x_{1}, \ldots, x_{n}\right) Q_{\pi}\left(x_{1}, \ldots, x_{n}\right) d^{\times} x_{1} \cdots d^{\times} x_{n}
\end{aligned}
$$

Comme dans le cas $\pi=\mathrm{St}_{1} \times \xi \mathrm{St}_{2 h+1} \times \xi \mathrm{St}_{2 k+1}$, on peut interpréter le terme $2^{\frac{\operatorname{card}(\operatorname{Coup}(x))}{2}}$ de façon suivante :

$$
2^{\frac{\operatorname{card}(\operatorname{Coup}(\chi))}{2}}=\sum_{\substack{w \in\left[W / W^{L}\right] \\ w \eta=\chi}} \frac{\operatorname{card}\left\{w^{\prime} \in W ; w^{\prime} w \in\left[W / W^{L}\right], w^{\prime} w \eta=w \eta, \theta\left(w^{\prime}\right)=w^{\prime}\right\}}{\operatorname{card}\left\{w^{\prime} \in W ; w^{\prime} w \in\left[W / W^{L}\right], w^{\prime} w \eta=w \eta\right\}}
$$

On en déduit le résultat suivant, qui n'est autre que la proposition 8.26 :

\section{Proposition 9.8. - On a l'égalité :}

$$
Q_{\pi}=\sum_{\substack{w \in\left[W / W^{L}\right], \theta(w \eta)=w \eta}} \frac{\operatorname{card}\left\{w^{\prime} \in W ; w^{\prime} w \in\left[W / W^{L}\right], w^{\prime} w \eta=w \eta, \theta\left(w^{\prime}\right)=w^{\prime}\right\}}{\operatorname{card}\left\{w^{\prime} \in W ; w^{\prime} w \in\left[W / W^{L}\right], w^{\prime} w \eta=w \eta\right\}} Q_{w \eta}^{\mathrm{sym}}
$$




\section{CHAPITRE 10}

\section{EN GUISE DE CONCLUSION}

Dans ce dernier chapitre, on écrit $\mathbf{H}$ pour $\mathbf{S p}(2 n)$ et $\mathbf{G}$ pour $\mathbf{G L}(2 n+1)$. Nous

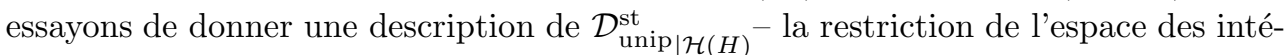
grales orbitales unipotentes stables à l'algèbre de Hecke. Cette description est basée sur les calculs effectués dans les chapitres précédents.

\subsection{Description de $\mathcal{D}_{\text {unip } \mid \mathcal{H}(H)}^{\text {st }}$}

Nous supposons que la caractéristique résiduelle de $F$ est assez grande. Sous cette hypothèse, Waldspurger a construit dans [Wa2] explicitement une base de $\mathcal{D}_{\text {unip }}^{\text {st }}$ que l'on va noter $\mathcal{B}$. Ses éléments sont paramétrés par :

- un entier naturel $k$ tel que $k(k+1) \leqslant n$;

- une représentation irréductible $\rho$ de $W\left(C_{n-k(k+1)}\right)$, le groupe de Weyl de type $C_{n-k(k+1)}$

Notons $\mathcal{I}^{\text {st }}(n)$ l'ensemble de tels paramètres et soit $I_{k, \rho}$ l'élément correspondant à $(k, \rho)$. Posons pour $k(k+1) \leqslant n, \mathcal{I}_{k}^{\text {st }}(n)=\left\{(k, \rho) \in \mathcal{I}^{\text {st }}(n)\right\}$ de sorte qu'on a une partition de $\mathcal{I}^{\text {st }}(n)$ :

$$
\mathcal{I}^{\text {st }}(n)=\cup_{k, k(k+1) \leqslant n} \mathcal{I}_{k}^{\text {st }}(n)
$$

Nous nous attendons à une réponse positive à la question suivante :

\section{RÉSULTATS ESPÉRÉS 1}

(1) -Annulation-Pour tout $(k, \rho) \in A^{\text {st }}$ tel que $k \neq 0$, la restriction de $I_{k, \rho} \grave{a}$ $\mathcal{H}(H)$ est nulle.

(2) -Base proposée pour $\mathcal{D}_{\text {unip } \mid \mathcal{H}(H)}^{\mathrm{st}}$ - Les restrictions à $\mathcal{H}(H)$ des éléments $I_{0, \rho}$,

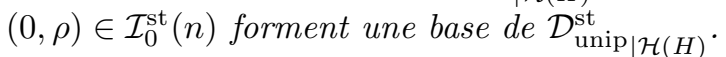

(3) -Transformées de Satake des éléments de $\mathcal{B}$ - Pour tout $(0, \rho) \in I_{0}^{\text {st }}(n)$, il existe une fonction rationnelle $c_{\rho}$ en $n$ variables, invariante par le groupe de $W e y l W\left(C_{n}\right)$, sans pôle au voisinage de $\widehat{T}_{u}$ telle que pour tout $f \in \mathcal{H}(H)$, on ait l'égalité :

$$
I_{k, \rho}(f)=\int_{\widehat{T}_{u}} S(f)\left(x_{1}, \ldots, x_{n}\right) c_{\rho}\left(x_{1}, \ldots, x_{n}\right) d^{\times} x_{1} \cdots d^{\times} x_{n} .
$$

On a vu que ces assertions sont vérifiées pour le groupe $\mathbf{S p}(4)$, cf. chapitre 3. 


\subsection{Analogie avec les traces tordues compactes}

Rappelons que l'on s'intéressait à calculer les traces tordues compactes des représentations $\pi$ de $G L(2 n+1, F)$ paramétrées par l'ensemble :

$$
\mathcal{A}^{\text {st }}(n)=\left\{\begin{array}{c}
\alpha^{+}, \alpha^{-}, \beta \text { des partitions telles que : } \\
\bullet \\
\qquad\left(\alpha^{+}\right)+S\left(\alpha^{-}\right)+2 S(\beta)=2 n+1 ; \\
\bullet \text { les partitions } \alpha^{+}, \alpha^{-} \text {sont constituées } \\
\text { de nombres impairs, sans multiplicités } ; \\
\bullet \text { le nombre de termes de } \alpha^{-} \text {est pair. }
\end{array}\right\}
$$

On espère que l'assertion suivante est vérifiée :

RÉSUltats ESPÉRÉS 2. - Pour tout $\left(\alpha^{+}, \alpha^{-}, \beta\right) \in \mathcal{A}^{\text {st }}(n)$, soit $\pi$ la représentation correspondante, il existe une fonction rationnelle $c_{\pi}$ en $n$ variables, invariante par le groupe de Weyl $W\left(C_{n}\right)$, sans pôle au voisinage de $\widehat{T}_{u}$ telle que pour tout élément $f$ de l'algèbre de Hecke de $G L(2 n+1, F)$ on ait l'identité :

$$
\operatorname{tr}_{\theta-c} \pi(f)=\int_{\widehat{T}_{u}} p(S(f))\left(x_{1}, \ldots, x_{n}\right) c_{\pi}\left(x_{1}, \ldots, x_{n}\right) d^{\times} x_{1} \cdots d^{\times} x_{n}
$$

Il a été démontré dans cette thèse que cela est vrai dans un certain nombre de cas particuliers :

- Si c'est vrai pour un triplet $\left(\alpha_{0}^{+}, \alpha_{0}^{-}, \varnothing\right)$ relatif à $n_{0}=S\left(\alpha^{+}\right)+S\left(\alpha^{-}\right)$, alors c'est vrai aussi pour tout triplet de la forme $\left(\alpha_{0}^{+}, \alpha_{0}^{-}, \beta\right)$, cf. chapitre 5 ;

- C'est vrai si $\left(\alpha^{+}, \alpha^{-}, \varnothing\right)$ est tel que $\alpha=\alpha^{+} \cup \alpha^{-}$a au plus trois termes, et s'il y en trois, un terme est égal à $1, c f$. chapitres $6,7,8$, et 9 .

Comme corollaire de ces résultats, on a :

Proposition 10.1. - L'assertion précédente est vraie pour $n=1,2,3$ et 4.

En effet, le premier cas qui n'est pas inclus dans ces calculs est le cas $\left(\alpha^{+}, \alpha^{-}, \varnothing\right)=$ $((5,3),(3), \varnothing)$ qui correspond à $n=5$.

Dans le cas où $\beta=\varnothing$, les résultats des chapitres 6 à 10 nous permettent de conjecturer la forme de la fonction $c_{\pi}$. Soit donc un triplet $\left(\alpha^{+}, \alpha^{-}, \varnothing\right) \in \mathcal{A}^{\text {st }}(n)$ et $\pi$ la représentation correspondante. Notons $\mathbf{R}=\mathbf{L V}$ le sous-groupe parabolique correspondant à la partition $\left(\alpha_{1}^{+}, \alpha_{2}^{+}, \ldots, \alpha_{1}^{-}, \alpha_{2}^{-}, \ldots\right)$ de $2 n+1$ et notons $\eta$ le caractère :

$$
\left(\frac{\alpha_{1}^{+}-1}{2}, \ldots, \frac{1-\alpha_{1}^{+}}{2}, \frac{\alpha_{2}^{+}-1}{2}, \ldots, \frac{\alpha_{1}^{-}-1}{2}+\varepsilon, \ldots, \frac{1-\alpha_{1}^{-}}{2}+\varepsilon, \frac{\alpha_{2}^{-}-1}{2}+\varepsilon, \ldots\right)
$$

On espère que :

$$
c_{\pi}=\sum_{\substack{w \in\left[W / W^{L}\right] \\ \theta(w \eta)=w \eta}} \frac{\operatorname{card}\left\{w^{\prime} \in W ; w^{\prime} w \in\left[W / W^{L}\right], w^{\prime} w \eta=w \eta, \theta\left(w^{\prime}\right)=w^{\prime}\right\}}{\operatorname{card}\left\{w^{\prime} \in W ; w^{\prime} w \in\left[W / W^{L}\right], w^{\prime} w \eta=w \eta\right\}} Q_{w \eta}^{\mathrm{sym}}
$$

où $Q_{w \eta}^{\text {sym }}$ est la fonction définie en section 8.3.2. 


\subsection{Comparaison entre $\mathbf{S p}(2 n)$ et $\mathbf{G L}(2 n+1, F)$}

La conjecture de transfert pour l'endoscopie tordue conduit à espérer la propriété suivante :

RÉSULTATS ESPÉRÉS 3. - Les espaces engendrés par les fonctions $c_{\pi}$ et $c_{\rho}$ coïncident.

Notons que cette assertion a été démontrée en 4.5 dans le cas où $n=2$. Nous allons donner une assertion plus descriptive en précisant comment extraire des fonctions $c_{\pi}$ une base de l'espace engendré par celles-ci. On remarque en effet que l'ensemble $\mathcal{A}^{\text {st }}(n)$ a un plus grand nombre d'éléments que l'ensemble $\mathcal{I}_{0}^{\text {st }}(n)$, qui comme on a dit, est susceptible de paramétrer une base de $\mathcal{D}_{\text {unip } \mid \mathcal{H}(H)}^{\text {st }}$.

Soit $\left(\alpha^{+}, \alpha^{-}, \beta\right) \in \mathcal{A}^{\text {st }}(n)$, notons $\alpha$ la partition obtenue par réunion des partitions $\alpha^{+}, \alpha^{-}$. Par hypothèse, chaque entier (strictement) positif apparaît avec une multiplicité 0,1 ou 2 dans $\alpha$, posons :

$$
a_{\text {imp }}(\alpha)=\left\{i \in \mathbb{N}^{*} ; i \text { de multiplicité } 1 \text { dans } \alpha\right\}
$$

Définissons l'application $\varepsilon$ de la façon suivante :

$$
\begin{aligned}
\varepsilon: a_{\mathrm{imp}}(\alpha) & \longrightarrow\{ \pm 1\} \\
i & \longmapsto(i)=\left[\begin{array}{rl}
1 & \text { si } i \in \alpha^{+} \\
-1 & \text { si } i \in \alpha^{-}
\end{array}\right.
\end{aligned}
$$

Écrivons $a_{\text {imp }}(\alpha)=\left\{i_{1}>i_{2}>\cdots>i_{m}\right\}$ et posons :

$$
\begin{aligned}
N\left(\alpha^{+}, \alpha^{-}, \beta\right)= & \operatorname{card}\left\{l ; l=1, \ldots, m, \quad \text { t.q. } l \text { pair et } \varepsilon\left(i_{l}\right)=-1\right\} \\
& -\operatorname{card}\left\{l ; l=1, \ldots, m, \quad \text { t.q. } l \text { impair et } \varepsilon\left(i_{l}\right)=-1\right\}
\end{aligned}
$$

On pose :

$$
\mathcal{A}_{0}^{\text {st }}(n)=\left\{\left(\alpha^{+}, \alpha^{-}, \beta\right) \in \mathcal{A}^{\text {st }}(n) ; N\left(\alpha^{+}, \alpha^{-}, \beta\right)=0 \text { ou }-1\right\}
$$

On va prouver le lemme suivant :

Lemme 10.2. - Les ensembles $\mathcal{I}_{0}^{\text {st }}(n)$ et $\mathcal{A}_{0}^{\text {st }}(n)$ ont même cardinal.

Démonstration. - Par définition, l'ensemble $\mathcal{I}_{0}^{\text {st }}(n)$ est paramétré par l'ensemble des classes d'isomorphie de représentations irréductibles de $W\left(C_{n}\right)$, lui-même en bijection avec l'ensemble des doubles partitions de $n$ :

$$
\mathcal{P}_{2}(n)=\{(\alpha, \beta) ; S(\alpha)+S(\beta)=n\}
$$

Notons $p_{2}(n)$ le nombre de telles doubles partitions. On note $\mathcal{P}(n)$ l'ensemble des partitions de $n$ et $p(n)$ son cardinal. On a une égalité élémentaire de séries formelles :

$$
\sum_{n=0}^{\infty} p(n) T^{n}=\frac{1}{\prod_{i=1}^{\infty}\left(1-T^{i}\right)}
$$


On vérifie facilement que :

$$
\sum_{n=0}^{\infty} p_{2}(n) T^{n}=\left(\sum_{n=0}^{\infty} p(n) T^{n}\right)^{2}
$$

d'où,

$$
\sum_{n=0}^{\infty} p_{2}(n) T^{n}=\left(\frac{1}{\prod_{i=1}^{\infty}\left(1-T^{i}\right)}\right)^{2}
$$

Posons, pour $n \in \mathbb{N}$,

$$
\begin{aligned}
& \mathcal{P}_{2}^{0}(2 n+1)=\left\{\begin{array}{cl}
\bullet & S\left(\alpha^{+}\right)+S\left(\alpha^{-}\right)=2 n+1 \\
& \bullet \text { les partitions } \alpha^{+}, \alpha^{-} \text {sont constituées } \\
\left(\alpha^{+}, \alpha^{-}\right) ; & \text {de nombres impairs, sans multiplicités } ; \\
\bullet & \text { le nombre de termes de } \alpha^{-} \text {est pair; } \\
\bullet & N\left(\alpha^{+}, \alpha^{-}, \varnothing\right)=0 \text { ou }-1
\end{array}\right\} \\
& =\left\{\left(\alpha^{+}, \alpha^{-}\right) ;\left(\alpha^{+}, \alpha^{-}, \varnothing\right) \in \mathcal{A}_{0}^{\text {st }}(n)\right\}
\end{aligned}
$$

et notons $c(n)$ le cardinal de cet ensemble.

Soit $a(n)$ le cardinal de l'ensemble $\mathcal{A}_{0}^{\text {st }}(n)$. Montrons que :

$$
\sum_{n=0}^{\infty} a(n) T^{n}=\left(\sum_{n=0}^{\infty} c(n) T^{n}\right)\left(\sum_{n=0}^{\infty} p(n) T^{n}\right)
$$

En effet, pour $n$ fixé on peut décomposer l'ensemble $\mathcal{A}_{0}^{\text {st }}(n)$ en union disjointe des ensembles $\left\{\left(\alpha^{+}, \alpha^{-}, \beta\right) \in \mathcal{A}_{0}^{\text {st }}(n) \mid S\left(\alpha^{+}\right)+S\left(\alpha^{-}\right)=2 k+1, S(\beta)=n-k\right\}$. Ces ensembles sont en bijection avec $\mathcal{P}_{2}^{0}(2 k+1) \times \mathcal{P}(n-k)$ (par application $\left(\alpha^{+}, \alpha^{-}, \beta\right) \mapsto$ $\left(\left(\alpha^{+}, \alpha^{-}\right), \beta\right)$, donc de cardinal $c(k) p(n-k)$. On en déduit $a(n)=\sum_{k=0}^{n} c(k) p(n-k)$, d'où l'égalité cherchée.

On obtient :

$$
\sum_{n=0}^{\infty} a(n) T^{n}=\left(\sum_{n=0}^{\infty} c(n) T^{n}\right)\left(\frac{1}{\prod_{i=1}^{\infty}\left(1-T^{i}\right)}\right)
$$

Il nous suffit donc de montrer que pour tout $n$,

$$
c(n)=p(n)
$$

Posons :

$$
\mathcal{P}^{0}(2 n+1)=\{\alpha \in \mathcal{P}(2 n+1) ; \text { à termes impairs, de multiplicités } \leqslant 2\}
$$

Pour tout entier positif $k$, posons :

$$
\mathcal{P}^{0}(2 n+1, k)=\left\{\alpha \in \mathcal{P}^{0}(2 n+1) ; \operatorname{card} a_{\mathrm{imp}}(\alpha)=2 k+1\right\}
$$

On note $\mathcal{E}(k)$ l'ensemble des applications

$$
\varepsilon:\{1,2, \ldots, 2 k+1\} \longrightarrow\{ \pm 1\}
$$


telles que $\operatorname{card}\{i$ pair; $\varepsilon(i)=-1\}-\operatorname{card}\{i \operatorname{impair} ; \varepsilon(i)=-1\}=0$ ou -1 . Soit $(\alpha, \varepsilon) \in$ $\mathcal{P}^{0}(2 n+1, k) \times \mathcal{E}(k)$ et écrivons :

$$
a_{\text {imp }}(\alpha)=\left\{i_{1}>i_{2}>\cdots>i_{2 k+1}\right\}
$$

Notons $\operatorname{mult}_{i}(\alpha)$ pour la multiplicité de $i$ dans $\alpha$. On pose :

$$
\begin{aligned}
& \mu^{+}=\left\{i \in \mathbb{N}^{*} ; \operatorname{mult}_{i}(\alpha)=2\right\} \cup\left\{i_{j} \in a_{\mathrm{imp}}(\alpha) ; \operatorname{mult}_{i_{j}}(\alpha)=1 \text { et } \varepsilon(j)=1\right\} \\
& \mu^{-}=\left\{i \in \mathbb{N}^{*} ; \operatorname{mult}_{i}(\alpha)=2\right\} \cup\left\{i_{j} \in a_{\mathrm{imp}}(\alpha) ; \operatorname{mult}_{i_{j}}(\alpha)=1 \text { et } \varepsilon(j)=-1\right\}
\end{aligned}
$$

Posons :

$$
\left(\alpha^{+}, \alpha^{-}\right)=\left[\begin{array}{ll}
\left(\mu^{+}, \mu^{-}\right) & \text {si } \operatorname{card}\left(\mu^{-}\right) \text {est pair } \\
\left(\mu^{-}, \mu^{+}\right) & \text {si } \operatorname{card}\left(\mu^{-}\right) \text {est impair }
\end{array}\right.
$$

On vérifie que l'application $(\alpha, \varepsilon) \mapsto\left(\alpha^{+}, \alpha^{-}\right)$définie ci-dessus établit une bijection entre $\cup_{k \in \mathbb{N}} \mathcal{P}^{0}(2 n+1, k) \times(\mathcal{E}(k) /\{ \pm 1\})$ (le groupe $\{ \pm 1\}$ agit de façon évidente sur $\mathcal{E}(k))$ et $\mathcal{A}_{0}^{\text {st }}(n)$. Soient $d(n, k), e(k)$ les cardinaux des ensembles $\mathcal{P}^{0}(2 n+1, k)$ et $\mathcal{E}(k) /\{ \pm 1\}$ respectivement. On a donc l'égalité :

$$
c(n)=\sum_{k \in \mathbb{N}} d(n, k) e(k)
$$

On a :

FAIT 1. - Le nombre e $(k)$ est égal à $\left(\begin{array}{c}2 k+1 \\ k\end{array}\right)$, le coefficient de $X^{k}$ dans $(1+X)^{2 k+1}$.

En effet, le quotient $\mathcal{E}(k) /\{ \pm 1\}$ est en bijection avec l'ensemble des

$$
\varepsilon:\{1,2, \ldots, 2 k+1\} \longrightarrow\{ \pm 1\}
$$

telles que $\operatorname{card}\{i$ pair; $\varepsilon(i)=-1\}-\operatorname{card}\{i$ impair; $\varepsilon(i)=-1\}=0$. Cet ensemble est union disjointe, pour $j=0, \ldots k$, des $\varepsilon$ tels que $\operatorname{card}\{i$ pair; $\varepsilon(i)=-1\}=$ $\operatorname{card}\{i \operatorname{impair} ; \varepsilon(i)=-1\}=j$. Un tel ensemble est de cardinal $\left(\begin{array}{c}k \\ j\end{array}\right)\left(\begin{array}{c}k+1 \\ j\end{array}\right)$. Mais $\sum_{j=0}^{k}\left(\begin{array}{c}k \\ j\end{array}\right)\left(\begin{array}{c}k+1 \\ j\end{array}\right)=\left(\begin{array}{c}2 k+1 \\ k\end{array}\right)$.

FAIT 2. - Le nombre $d(n, k)$ est égal au coefficient de $X^{2 k+1} T^{2 n+1}$ dans la série formelle :

$$
S(X, T)=\prod_{i \in \mathbb{N}, i \text { impair }}\left(1+X T^{i}+T^{2 i}\right)
$$

Posons :

$$
R(X, T)=\prod_{i \in \mathbb{N}, i \text { impair }}\left(1+\left(X+X^{-1}\right) T^{i}+T^{2 i}\right)
$$

Développons $R(X, T)$ suivant $X+X^{-1}$ et $T$, on obtient donc une somme sur les puissances $\left(X+X^{-1}\right)^{j} T^{l}$. Développons ensuite les $\left(X+X^{-1}\right)^{j}$ en des sommes des puissances de $X$. Considérons le coefficient $X T^{2 n+1}$ dans $R(X, T)$ développé de cette façon. En comparant la puissance de $T$, puis de $X$, il est donc égal à la somme sur les entiers positifs $j$ du produit des coefficients

- de $X^{j} T^{2 n+1}$ dans $S(X, T)$;

- et de $X$ dans $\left(X+X^{-1}\right)^{j}$. 
Pour $j$ pair, on vérifie facilement que le premier coefficient est nul. Pour $j$ impair, en écrivant $j=2 k+1$, le premier coefficient est $d(n, k)$, le second est $\left(\begin{array}{c}2 k+1 \\ k\end{array}\right)$ ou encore $e(k)$. Le coefficient de $X T^{2 n+1}$ dans $R(X, T)$ est donc égal à

$$
\sum_{k \in \mathbb{N}} d(n, k) e(k)
$$

On a ainsi montré que le nombre $c(n)$ est égal au coefficient de $X T^{2 n+1}$ dans $R(X, T)$. D'autre part, on a le résultat suivant :

FAIT 3. - On a l'égalité :

$$
R(X, T)=\frac{1+\sum_{i=1}^{\infty} T^{i^{2}}\left(X^{i}+X^{-i}\right)}{\prod_{i=1}^{\infty}\left(1-T^{2 i}\right)}
$$

C'est le théorème 352 , page 282 dans $[\mathbf{H}-\mathbf{W}]$.

Le nombre $c(n)$ est donc égal au coefficient de $X T^{2 n+1}$ dans le développement en série formelle de

$$
\frac{1+\sum_{i=1}^{\infty} T^{i^{2}}\left(X^{i}+X^{-i}\right)}{\prod_{i=1}^{\infty}\left(1-T^{2 i}\right)}
$$

c'est-à-dire au coefficient de $T^{2 n+1}$ dans le développement de $T / \prod_{i=1}^{\infty}\left(1-T^{2 i}\right)$, qui est égal à $p(n)$, comme attendu. Le lemme en découle.

En 10.1, on a décomposé $\mathcal{I}^{\text {st }}(n)$ en union disjointe de sous-ensembles $\mathcal{I}_{k}^{\text {st }}(n)$. Cette décomposition provient d'une correspondance de Springer généralisée, qui intervient comme un deus ex machina dans [Wa2]. Cela suggère de décomposer $\mathcal{A}^{\text {st }}(n)$ en faisant intervenir, sinon la correspondance de Springer généralisée, du moins des formules qui en soient réminiscentes. Notre définition de l'entier $N\left(\alpha^{+}, \alpha^{-}, \beta\right)$ est ainsi calquée sur une formule intervenant dans la correspondance de Springer généralisée pour le groupe $\mathbf{S O}(2 n+1)$, cf. [Wa2, p. 328]. Pour tout entier $N \geqslant 0$, posons :

$$
\mathcal{A}_{N}^{\text {st }}(n)=\left\{\left(\alpha^{+}, \alpha^{-}, \beta\right) \in \mathcal{A}^{\text {st }}(n) ; N\left(\alpha^{+}, \alpha^{-}, \beta\right)=N \text { ou }-1-N\right\}
$$

Alors $\mathcal{A}^{\text {st }}(n)$ est réunion $\operatorname{des} \mathcal{A}_{N}^{\text {st }}(n)$ pour les $N$ tels que $2 N(N+1) \leqslant n$. Cette décomposition ressemble à celle de $\mathcal{I}^{\text {st }}(n)$ donnée en 10.1. Le lemme 10.2 rend alors plausible l'assertion suivante :

RÉSUltats ESPÉRÉS 4. - Lorsque les éléments $\left(\alpha^{+}, \alpha^{-}, \beta\right)$ parcourent $\mathcal{A}_{0}^{\text {st }}(n)$, les fonctions $c_{\pi}$ forment une base de l'espace engendré par toutes les fonctions $c_{\pi}$

Reconnaissons toutefois que nous ne disposons d'aucun exemple pour étayer cette assertion. 


\section{BIBLIOGRAPHIE}

[As1] M. AssEM - «Some results on unipotent orbital integrals», Compositio Math. 78 (1991), p. 37-78.

[As2] , «Unipotent orbital integrals of spherical functions on $p$-adic $4 \times 4$ symplectic groups », J. reine angew. Math. 437 (1993), p. 181-216.

[Car] R.W. CARTER - Finite groups of Lie type. Conjugacy classes and complex characters, Wiley Classics Library, John Wiley and Sons, Ltd., Chichester, 1993, Reprint of the 1985 original.

[Clo] L. Clozel - «The fundamental lemma for stable base change », Duke Math. J. 61 (1990), no. 1, p. 255-302.

[H-W] G.H. Hardy \& E.M. Wright - An introduction to the theory of numbers, fifth éd., The Clarendon Press, Oxford University Press, New York, 1979.

[K-S] R.E. Kottwitz \& D. Shelstad - Foundations of twisted endoscopy, Astérisque, vol. 255, Société Mathématique de France, Paris, 1999.

[Mac] I.M. MACDONALD - Spherical functions on a group of p-adic type, Ramanujan Institute for Advanced Study in Mathematics, Madras, 1971.

[Ree] M. REEDER - « Nonstandard intertwining operators and the structure of unramified principal series representations », Forum Math. 9 (1997), no. 4, p. 457516.

[Ro1] J.D. RogAwSKI - « On modules over the Hecke algebra of a p-adic group », Invent. Math. 79 (1985), no. 3, p. 443-465.

[Ro2] « "Trace Paley-Wiener theorem in the twisted case», Trans. Amer. Math. Soc. 309 (1988), no. 1, p. 215-229.

[Van] G. VAN DIJK - «Computation of certain induced characters of $p$-adic groups », Math. Ann. 199 (1972), p. 229-240. 
[Wa1] J.-L. WALDSPURGER - «Sur les intégrales orbitales tordues pour les groupes linéaires : un lemme fondamental», Canad. J. Math. 43 (1991), no. 4, p. 852896.

[Wa2] - Intégrales orbitales nilpotentes et endoscopie pour les groupes classiques non ramifiés, Astérisque, vol. 269, Société Mathématique de France, Paris, 2001. 